\title{
Neuromodulation of the dorsal root ganglion in experimental chronic neuropathic pain
}

Citation for published version (APA):

Franken, G. (2020). Neuromodulation of the dorsal root ganglion in experimental chronic neuropathic pain: efficacy and mechanisms of action. [Doctoral Thesis, Maastricht University]. Ipskamp. https://doi.org/10.26481/dis.20201203gf

Document status and date:

Published: 01/01/2020

DOI:

10.26481/dis.20201203gf

Document Version:

Publisher's PDF, also known as Version of record

\section{Please check the document version of this publication:}

- A submitted manuscript is the version of the article upon submission and before peer-review. There can be important differences between the submitted version and the official published version of record. People interested in the research are advised to contact the author for the final version of the publication, or visit the DOI to the publisher's website.

- The final author version and the galley proof are versions of the publication after peer review.

- The final published version features the final layout of the paper including the volume, issue and page numbers.

Link to publication

\footnotetext{
General rights rights.

- You may freely distribute the URL identifying the publication in the public portal. please follow below link for the End User Agreement:

www.umlib.nl/taverne-license

Take down policy

If you believe that this document breaches copyright please contact us at:

repository@maastrichtuniversity.nl

providing details and we will investigate your claim.
}

Copyright and moral rights for the publications made accessible in the public portal are retained by the authors and/or other copyright owners and it is a condition of accessing publications that users recognise and abide by the legal requirements associated with these

- Users may download and print one copy of any publication from the public portal for the purpose of private study or research.

- You may not further distribute the material or use it for any profit-making activity or commercial gain

If the publication is distributed under the terms of Article $25 \mathrm{fa}$ of the Dutch Copyright Act, indicated by the "Taverne" license above, 


\section{Neuromodulation of the Dorsal Root Ganglion in Experimental Chronic}

Neuropathic Pain

Efficacy and Mechanisms of Action

Glenn Franken 
C 2020 Glenn Franken, Maastricht, 2020

Cover design: Glenn Franken, Perla Douven en Margot Heijmans ISBN 978-94-6421-136-8

Printed by: Ipskamp Printing

All rights reserved. No part of this thesis may be reproduced, stored in a retrieval system of any nature, or transmitted in any form or by any means, electronic, mechanical, photocopying, recording, or otherwise, without permission of the author 


\section{Universiteit Maastricht}

\section{Neuromodulation of the Dorsal Root Ganglion in Experimental Chronic Neuropathic Pain:}

Efficacy and Mechanisms of Action

\section{ACADEMISCH PROEFSCHRIFT}

Ter verkrijging van de graad van doctor aan de Universiteit Maastricht,

op gezag van de Rector Magnificus, Prof. Dr. Rianne M. Letschert

volgens het besluit van het College van Decanen,

in het openbaar te verdedigen

op donderdag 3 december 2020 om 12.00 uur

door

Glenn Franken 


\section{Promotoren}

Prof. Dr. E.A.J. Joosten

Prof. Dr. J.H.M van Zundert

\section{Co-promotor}

Prof. Dr. A.L. Liem (Medisch Centrum Jan van Goyen, Amsterdam)

\section{Beoordelingscommissie}

Prof. Dr. W.F.F.A. Buhre (voorzitter)

Prof. Dr. Y. Temel

Prof. Dr. J.H.H.J. Prickaerts

Dr. J.W. Kallewaard (Rijnstate Ziekenhuis, Arnhem)

Prof. Dr. K.C.P. Vissers (Radboud UMC, Nijmegen)

Financial support for the completion of this thesis was partially provided in the form of a research grant from:

\section{Abbott}


Table of Contents

Chapter $1 \quad$ General Introduction

Chapter $2 \quad$ Spinal Cord Stimulation in Chronic Neuropathic Pain: 25 Mechanisms of Action, New Locations, New Paradigms

Published. Joosten and Franken. PAIN 2020; 161 Suppl 1:S104S13.

Chapter 3 Dorsal Root Ganglion Stimulation in Experimental Painful

Diabetic Peripheral Neuropathy: Burst versus Conventional Stimulation Paradigm

Published. Franken et al. Neuromodulation: Technology at the Neural Interface 2019; 22(8): 943-50.

Chapter $4 \quad$ Nonlinear Relation between Burst Dorsal Root Ganglion

Stimulation Amplitude and Behavioral Outcome in an Experimental Model of Painful Diabetic Peripheral Neuropathy Published. Franken et al. Neuromodulation: Technology at the Neural Interface 2020; 23(2): 158-66.

Chapter 5 A Possible Mechanism of Conventional Dorsal Root Ganglion Stimulation in Pain Relief: Quantitative Immunocytochemical Analysis of Intracellular GABA in DRG Neurons Submitted.

Chapter 6 The Use of CatWalk Gait Analysis for Assessing Chronic Neuropathic Pain in Rats Treated with Pulsed Radiofrequency Submitted.

Chapter 7 Summary, Discussion, and Conclusion

Appendix

Valorization 

CHAPTER

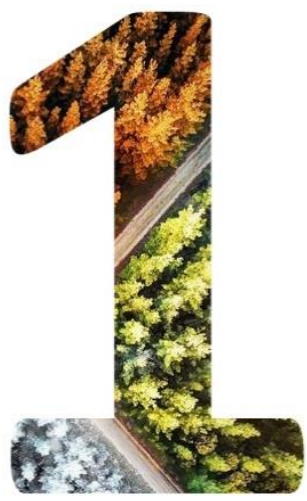

General Introduction 


\subsection{Neuropathic pain: Definition}

Neuropathic pain is a complex, heterogeneous disorder that affects approximately $8 \%$ of the total adult human population and comes with significant burden for both the patient and healthcare system. The international association for the study of pain (IASP) defines neuropathic pain as: "pain that arises as a direct consequence of a lesion or disease affecting the somatosensory system" [1].

The somatosensory system is a complex system involved in the perception of pain, touch, pressure, temperature, vibration and movement. A lesion or disease affecting this system leads to altered or enhanced signal transmission in either the peripheral (PNS) and/or central nervous system (CNS), which is a completely natural and healthy reaction of the body in response to a damaging insult. It is when this process is deregulated over a longer period of time, that neuropathic pain develops. In patients with chronic neuropathic pain (defined as neuropathic pain lasting for more than 3 months and classified as a disease under ICD-11 [2]), the body acts inadequate and non-functional to the initially obtained damage due to sensitization of the nociceptive network at the level of the spinal cord. Neuropathic pain can both originate in the PNS as well as the CNS. Peripheral neuropathic pain typically results from a lesion of the PNS, caused by mechanical trauma (peripheral nerve injury/compression), metabolic diseases (e.g. Diabetes Mellitus (DM)), neurotoxic substances, infection, or tumor invasion, whereas central neuropathic pain is most commonly caused by spinal cord injury, stroke, or multiple sclerosis [3]. Neuropathic pain patients usually experience burning, electric, stabbing or tingling sensations, which arise either spontaneously or upon application of an external stimulus [4]. Stimulus-evoked neuropathic pain can be characterized with presence of hyperalgesia and allodynia. Hyperalgesia can be defined as increased pain from a stimulus that normally provokes pain, whereas allodynia can be defined as pain to a stimulus that does not normally provoke pain. Besides its manifestation in the clinic, mechanical and thermal hyperalgesia/allodynia are also commonly used in experimental animal studies as a measure of "pain". The collection of these symptoms make that neuropathic pain patients often suffer from a low healthrelated quality of life, have high amounts of drug prescriptions, sleep disturbances, and a 
high chance of developing anxiety and depression $[5,6]$. The pathophysiology underlying neuropathic pain is explained in greater detail below.

\subsection{Neuropathic pain: Pathophysiology}

The pathophysiology underlying neuropathic pain consists of a complex interplay between cellular and molecular processes across different parts of the somatosensory nervous system, including the peripheral nerves, the spinal cord, and the brain. The perception of pain starts in sensory nerve endings arising in the periphery, including the skin, muscles, joints and fascia, where they can react to environmental changes through specialized receptors including nociceptors (damaging or potentially damaging mechanical stimuli), mechanoreceptors (pressure or distortion), thermoreceptors (temperature), and chemoreceptors (chemical substances). When stimulated, these receptors can signal information to the spinal cord via $1^{\text {st }}$ order afferent neurons. First-order afferent neurons include thinly myelinated $A \delta$-fibers (transmission of sharp/quick pain signals), unmyelinated C-fibers (transmission of slow, dull pain signals) and thickly myelinated $A \beta$-fibers (transmission of touch signals). The sensory neuron somata for all sensory modalities and fiber types (so $A \beta-, A \delta$ - and C-fibers) are collected in a structure called the dorsal root ganglion (DRG). DRG's are located at each segmental level of the spinal column at the intersection of the peripheral and central nervous system. DRG neurons have a unique "pseudo-unipolar" design, with axons that divide in two separate branches (one branch going from the periphery to the cell body, and one branch going from the cell body to the spinal cord), connected by a T-junction which connects the branching point to the cell body. This T-junction can either act as an impediment to electrical impulses traveling from the periphery to the dorsal horn of the spinal cord, participate in the propagation of the electrical pulse, or act as a low-pass filter to electrical information passing through the DRG from the periphery [7]. The cell bodies that reside in the DRG were originally thought to only serve as metabolic storage "helpers" to peripheral processes. However, over the years, it has become clear that these DRG cell bodies act as a pivotal relay station in the nociceptive network and thus the pathogenesis of neuropathic pain [8]. Upregulation of multiple neuropeptides, receptors, ion channels, signal transduction molecules, synaptic vesicle proteins, and other 
factors involved in the development of neuropathic pain have been observed in the DRG in response to nerve injury and inflammation [9], indicating the importance of the DRG in the nociceptive cascade. These changes at the site of the DRG can lead to the production of ectopic, aberrant, discharges. It was calculated that more than $75 \%$ of these ectopic discharges were generated at the site of the DRG, whereas only $25 \%$ of the ectopic discharges were generated at the lesion or neuroma site, after peripheral nerve injury [10].

Ectopic discharges generated in the DRG can reach the dorsal horn of the spinal cord, where they can activate postsynaptic, $2^{\text {nd }}$ order projection neurons due to increased synaptic efficiency via modulation of among others glutamate, substance-p and BDNF [11, 12]. These $2^{\text {nd }}$ order projection neurons include both nociceptive-specific (NS) projection neurons located in the superficial dorsal horn (predominantly laminae I-II), but also wide dynamic range (WDR) projection neurons (i.e. $2^{\text {nd }}$ order neurons capable of responding to multiple types of somatosensory stimuli, including both noxious and innocuous input) located in the deeper laminae IV-VI. Additionally, ectopic firing induced at the DRG can induce microglia activation in the dorsal horn of the spinal cord, which, in turn, can also produce BDNF. Both this neuronal and microglia-derived BDNF can bind to its post-synaptic tyrosine kinase receptor B (trkB) [13], which in turn can interfere with the opening of postsynaptic NMDA channels important in the process of central sensitization $[14,15]$. Collectively, micro-environmental changes at the site of the DRG, together with the production of ectopic discharges and neuropeptides, can sensitize the transmission of afferent nociceptive input in the dorsal horn of the spinal cord.

Second-order projection neurons cross over to the contralateral site before they ascend to the brain in what is called the spinothalamic tract, after which these neurons synapse onto their respective $3^{\text {rd }}$ order neurons in predominantly the thalamus and brain stem. These $3^{\text {rd }}$ order neurons connect to multiple brain areas that play a role in the processing of pain signals. Collectively, these brain areas are commonly referred to as "the pain matrix", and include areas like the central amygdala, prefrontal cortex, anterior cingulate cortex, periaqueductal grey, thalamus, insula, inferior parietal cortex and the 
primary and secondary cortices. It is a strong interplay between these structures that causes the experience we refer to as "pain", and includes both the objective/discriminative as well as the affective components of the pain experience. Finally, after supraspinal integration of the pain signal, a feedback loop of descending serotonergic, noradrenergic and dopaminergic projections to the dorsal spinal horn further modulates and controls the spinal dorsal horn network [16].

\subsection{Treatment algorithm for neuropathic pain}

The standard treatment approach for managing neuropathic pain is to start with pharmacological interventions. Pregabalin and gabapentin (gabapentinoids: calcium channel alpha2-delta ligands), duloxetine (a serotonin-noradrenaline reuptake inhibitor) and a number of tricyclic antidepressants are recommended first-line treatments for neuropathic pain. Capsaicin patches, lidocaine patches, and tramadol (a $\mu$-opioid receptor agonist and serotonin-noradrenalin reuptake inhibitor (SNRI)) have less evidence and are mostly considered as second-line treatments. Stronger opioids such as oxycodone and morphine ( $\mu$ opioid receptor agonists) and botulinum toxin A (a potent neurotoxin) have only weak recommendations as third-line treatment options [17-22].

Despite the development and use of many pharmacological drugs and guidelines for the treatment of chronic neuropathic pain over the years, a substantial amount of neuropathic pain patients remain under- or untreated, with less than $50 \%$ of patients responding to pharmacological treatment [23]. These chronic pain patients are diagnosed with having refractory neuropathic pain. The Scottish Medicines Consortium specifies patients who suffer from refractory chronic pain as: "those who have not achieved adequate pain relief from, or have not tolerated, conventional first and second line treatments" [24]. Hence, the development of novel, last-resort interventional treatment therapies is crucial to also relief neuropathic pain in these refractory patients. Recent developments in the field of interventional neuromodulation have shown great promise for these patients. 


\subsection{Interventional neuromodulation in treatment of neuropathic pain}

Over the last decades, neuromodulation based on electrical stimulation has proven to be a valuable last-resort treatment option for patients with refractory neuropathic pain. With neuromodulation, nerve fiber activity is being altered through targeted delivery of an electrical stimulus to defined neurological sites in the body, as with neuropathic pain particular the spinal cord and DRG. This neuromodulation is aimed to interfere with nociceptive transmission and with that minimize pain transmission to and in the brain. This thesis will focus on neuromodulation of nociceptive transmission via targeting the Dorsal Root Ganglion (DRG). As mentioned in section 1.2, the DRG's play a crucial role in the pathogenesis of neuropathic pain. The unique, pseudo-unipolar structure of DRG neurons and the fact that the somata for all sensory modalities and fiber types reside in the DRG at each segmental level, makes this anatomical entity a very interesting target for neuromodulation. Two interventional techniques, namely dorsal root ganglion stimulation (DRGS) and pulsed radiofrequency (PRF) are investigated with focus on their pain relieving effect and mechanism of action in chronic neuropathic pain.

\subsection{1. (Pulsed) radiofrequency treatment}

Radiofrequency (RF) treatment is a frequently used therapy for treating neuropathic pain. During RF treatment, an electrode is transcutaneously placed on a peripheral nerve or DRG of interest, after which radiofrequency current is applied for a brief period of time (usually 2-8 minutes). This administration of radiofrequency current results in heating of the electrode tip (up until $80^{\circ} \mathrm{C}$ ) and as a result produces a lesion through the process of thermocoagulation. The lesioning or ablation of the neurological tissue interrupts the movement of impulses across the nerve, and thus effectively reduces pain perception [25, 26]. Although RF has been successful at reducing pain in multiple chronic pain conditions, several reports have pointed out its neurological side effects. These side effects vary from post-procedural pain and dysesthesias to sensory loss in the treated dermatomes and motor disturbances $[27,28]$. 
In order to minimize the undesired neurological side effects related to RF, Sluijter et al. were the first to describe an isothermal variant of RF named pulsed radiofrequency (PRF) in 1998 [29]. In contrast to the view that RF relies on heat-induced damage of small nociceptive C-fibers, Sluijter and colleagues proposed the idea that PRF, due to the high intensity electrical fields generated, has a neuromodulatory effect on DRG fibers and their nociceptive endings located in the dorsal horn of the spinal cord. During PRF treatment, tissue injury as a consequence of thermocoagulation is avoided by limiting the peak electrode temperature to $42^{\circ} \mathrm{C}$, which is considered the threshold temperature for neurodestruction, while at the same time applying higher voltages to maximize the delivery of electromagnetic force. This is achieved by administrating radiofrequency current in a pulsed-based fashion (2$4 \mathrm{~Hz}$ ), and alternating brief pulses $(20 \mathrm{~ms}$ ) of high voltage (45 V) with resting periods (480 ms) to dissipate the generated heat $[25,30]$.

Mechanistically, PRF adjacent to the DRG has been linked to cellular stress in DRG soma of small afferent neurons (A $\delta$ - and C-fibers) [31] and increased neural activity [32], excitatory neurotransmitter release [33], and micro-environmental changes [34, 35] in the dorsal horn of the spinal cord. Importantly, although the general consensus is that PRF relies on a neuromodulatory effect, the factor neurodestruction, albeit on a microscopic level, cannot be ruled out [30]. Multiple studies have shown that PRF adjacent to the DRG can cause small histological and morphological changes, like enlarged endoplasmic reticulum cisterns and increased number of cytoplasmic vacuoles [36, 37]. These changes seem to be most pronounced in C-fibers and are much milder when compared to RF-induced morphological changes.

Although a growing amount of literature suggests clinical efficacy of PRF adjacent to the DRG for neuropathic pain on the cervical [38], as well as lumbar [39-41] level, strong clinical evidence for the use of PRF adjacent to the DRG in neuropathic pain remains limited. PRF adjacent to the DRG also tends to provide positive outcomes in preclinical animal models of neuropathic pain in terms of mechanical and thermal hypersensitivity, although literature shows that the effects of PRF, the pain model used, electrode positioning, time frame, and 
stimulation settings are subjected to great diversity between research groups [33-35, 39, 4249]. Together, this creates the need for further validation of PRF adjacent to the DRG as a treatment for neuropathic pain on both the clinical and preclinical level. Also the addition of novel, objective behavioral tests are necessary to successfully validate and translate preclinical findings related to the effect of PRF and neuropathic pain.

\subsubsection{Spinal cord stimulation}

Chronic neuropathic pain can also be treated with use of neuromodulation of the thick $A \beta$-fibers in the spinal cord dorsal columns. Spinal cord stimulation (SCS) requires transcutaneous implantation of one or multiple leads into the epidural space on top of the dura mater surrounding the spinal cord. Electrical pulses are then applied to the dorsal columns of the spinal cord via an implantable pulse generator (IPG). SCS was developed based on the Gate-Control Theory as formulated by Melzack and Wall in 1965 [50]. The GateControl theory postulates that stimulation of large diameter, fast conducting, nonnociceptive touch affiliated $A \beta$-fibers can block nociceptive input from the smaller sized and slower conducting nociceptive A $\delta$ - and C-fibers in the dorsal horn of the spinal cord ("fast blocks slow" [51]), and thereby "close the spinal gate". Stimulation of large diameter A $\beta$ fibers was hypothesized to achieve this goal via activation of inhibitory interneurons located in the dorsal horn of the spinal cord. When activated, these interneurons release the inhibitory neurotransmitter gamma-amino-butyric acid (GABA) [52-55], which presynaptically modulate the incoming nociceptive signals from $A \delta$ - and C-fibers.

Over the years, SCS with conventional settings (Con-SCS; frequency $=30-80 \mathrm{~Hz}$, pulse width $=100-500 \mu \mathrm{s}$, amplitude above sensory threshold) has proven to be a valuable lastresort treatment option for a wide variety of neuropathic pain disorders, such as painful diabetic peripheral neuropathy (PDPN) [56, 57], complex regional pain syndrome (CRPS) [58], and failed back surgery syndrome (FBSS) $[59,60]$. Despite considerable improvements, there are limitations to the efficacy of Con-SCS. First, only $50-70 \%$ of patients achieve pain reductions of $\geq 50 \%[56,57,59,60]$. Second, the average pain reduction is restricted to approximately $50-60 \%[56,57,59,60]$. Third, Con-SCS is often unable to satisfactory and 
specifically stimulate difficult-to-reach areas, such as the extremities or the groin. Fourth, placement of the leads on top of the dorsal columns makes this therapy susceptible to postural variations due to changes in distance between stimulation lead and stimulation target, leading to unpleasant paresthesias and/or overstimulation [61]. Last, the energy consumption of SCS is relatively high, as there is significant energy loss to the local environment such as the cerebrospinal fluid (CSF), before the electrical energy (charge) reaches the spinal cord dorsal columns.

In an attempt to improve the efficacy of SCS, many novel stimulation waveforms have been developed over the years. Two prominent examples, high frequency SCS (HF-SCS) and Burst-SCS, were recently introduced in order to optimize the pain relieving effect of SCS. These two SCS modalities are typically delivered below sensory threshold, which has had huge impact in the field of SCS as this gives the opportunity to perform double-blind, placebocontrolled studies [62-65].

With HF-SCS, tonic stimulation is applied at a frequency of $1000 \mathrm{~Hz}-10.000 \mathrm{~Hz}$, a pulse width of approximately $30 \mu \mathrm{s}$, and an amplitude ranging from 1-5 mA [66]. The proposed working mechanisms underlying HF-SCS vary from temporal summation (multiple pulses build on each other to achieve neuronal activation), to a depolarization blockade induced by the high frequency stimulation, to pseudo-spontaneous or stochastic neuronal activity in the spinal pain gate due to desynchronization [67-69]. Yet, the optimal frequency of HF-SCS, as well as its superiority over Con-SCS, still needs to be determined, as clinical evidence suggests that multiple HF-SCS frequencies can cause significant pain relief $[65,66$, 70-74].

Burst stimulation administered at an interburst frequency of $40 \mathrm{~Hz}$, an intraburst frequency of $500 \mathrm{~Hz}$, a pulse width of $1 \mathrm{~ms}$, with pulses delivered at a $1 \mathrm{~ms}$ spike interval was first introduced by Dirk De Ridder [75] and later patented by Abbott Inc. as "BurstDR". Various clinical studies strongly suggest BurstDR to be superior to Con-SCS as it decreases pain intensity to a greater degree than Con-SCS [76, 77]. This superior effect might be attributed to the underlying mechanism of action of BurstDR-SCS, as it is hypothesized that 
BurstDR-SCS, besides targeting brain areas related to the location and intensity of pain (lateral pain pathway), also targets areas related to the emotional and affective components of pain (medial pain pathway) [76].

However, even with these novel SCS waveforms, the pain relieving effect of SCS as well as responder rates can be improved. Additionally, certain indications demand a more specific stimulation modality, such as extremity pain in PDPN, and groin pain. In view of this it interesting to note that selective stimulation of the DRG has developed over the years.

\subsubsection{Dorsal root ganglion stimulation}

Dorsal root ganglion stimulation (DRGS) was developed to overcome some of the drawbacks of SCS (see 1.4.2). With DRGS, leads are transcutaneously implanted in the epidural space on top of the dura mater surrounding the spinal cord, but are then advanced laterally through the intervertebral foramen, in order to place the lead over the DRG of interest. Since the first fully implanted DRGS system in 2011 by Liem and colleagues [78], DRGS has been successfully implemented for a wide variety of neuropathic pain disorders, including, but not limited to, axial back pain [79], leg and foot pain [78], CRPS [58], chest wall pain [80], post-amputation pain syndromes [81], and PDPN [82]. Yet, only one randomized clinical trial (RCT) on DRGS has been published to date [58]. This study found DRGS to be noninferior and superior to traditional SCS for treating chronic intractable pain of the lower limbs attributed to CRPS. Additionally, patients receiving DRGS were found to have less therapy habituation as compared to patients treated with traditional SCS at 9 and 12 months [83]. Also the amount, intensity, area size, and dependency on positional changes of paresthesias were found to be less with DRGS over traditional SCS [84], with some DRGS patients achieving paresthesia-free analgesia [85].

Mechanistically, it was initially assumed that DRGS engages the spinal mechanisms dependent on stimulation of non-nociceptive $A \beta$-fibers and GABA release in the dorsal horn of the spinal cord as occurs in Con-SCS. While a recent computational study indeed suggested that DRGS may provide pain relief by activating pain-gating mechanisms in the dorsal horn via repeated activation of large myelinated $(A \beta)$ afferents [86], another study found that the 
pain relieving effect of DRGS is not likely to be dependent on GABA release in the spinal dorsal horn at the L4-L6 lumbar level [87]. Experimental studies suggest that instead, DRGS suppresses excitability of neurons with predominantly slow-conducting fibers (A $\delta$ - and Cfibers) $[88,89]$. Because of the unique pseudo-unipolar design of DRG neurons, the DRG is likely to act as an impediment or low-pass filter to electrical impulses traveling from the peripheral nociceptor to the spinal cord in response to electrical stimulation $[7,88]$. Interestingly, a recent study by Du et al. reported an extensive GABAergic communication network between sensory neuron somata in the DRG. The sensory neurons in the DRG express major proteins required for GABA synthesis and release, and are capable of releasing GABA upon depolarization. From this it was proposed that this GABAergic system in the DRG acts as a second gate important in modulating the nociceptive input. This second gate is hypothesized to act in addition to the GABAergic modulation in the spinal cord as proposed in the Gate Control Theory (or first gate) [90]. The conduction block at the DRG is consistent with the observation that DRGS attenuates blood oxygen-level dependent (BOLD) signals of brain areas that are considered to be part of the pain matrix including thalamic nuclei, and cortical S1 and S2 that were increased by noxious hind-limb stimulation in rats [91].

Although promising, the therapeutic efficacy of DRGS should be confirmed and verified in additional large-scale RCTs focused at identified pain etiologies. Future experimental studies are also needed to unravel the underlying mechanisms of DRGS, including the possible role of a hypothesized second (GABAergic) gate. Then, the effect of new stimulation paradigms, combined with this new stimulation location (DRGS), needs to be further developed in order to improve the analgesic efficacy of DRGS.

\subsection{Aims and research questions}

The primary aim of this academic thesis is to investigate the efficacy and mechanisms of action of neuromodulation of the DRG in experimental chronic neuropathic pain. 


\section{RESEARCH QUESTIONS (RQ's)}

1. What are the recent developments in the field of SCS in neuropathic pain with focus on novel anatomical targets and paradigms?

2. Does the anti-nociceptive effect of Conventional DRGS and Burst DRGS differ over time in an experimental model of PDPN?

3. What is the effect of Burst DRGS amplitude on mechanical hypersensitivity in an experimental model of PDPN?

4. Does Conventional DRGS reduce intracellular GABA immunoreactivity in DRG somata?

5. Is PRF adjacent to the DRG effective in attenuating mechanical and thermal hypersensitivity and pain-related gait in a rat model of chronic neuropathic pain?

\subsection{Outline of the thesis}

RQ1 is addressed in Chapter 2, and summarizes the current literature on SCS, with focus on recent advancements in terms of novel stimulation locations (DRGS), and novel stimulation paradigms (such as Burst stimulation). In Chapter 3 and Chapter 4, we combined these two novel advancements and studied, for the first time, the effect of Burst-DRGS in an animal model of experimental PDPN.

More specifically, $R Q 2$, addressed in Chapter 3, investigates and compares the effect of Con-DRGS and Burst-DRGS on mechanical hypersensitivity during stimulation (wash-in and maximum effect: 30 minutes), and 30 minutes post-stimulation (wash-out).

In order to gain more insight into the effect of stimulation amplitude on Burst-DRGS efficacy, we then studied the behavioral effect of Burst-DRGS in response to various stimulation amplitudes in Chapter 4 (RQ3). Here, we titrated the optimal Burst-DRGS amplitude for maximum efficacy.

In RQ4, addressed in Chapter 5, we aimed to explore the working mechanism of ConDRGS. By use of quantitative immunocytochemical analysis on intracellular GABA 
immunoreactivity of sensory neuron somata located in the DRG, we tested the hypothesis that DRGS acts via diminishing intracellular GABA concentrations in sensory neuron somata located locally in the DRG.

Lastly, we tested the efficacy of PRF applied adjacent to the DRG on mechanical and thermal hypersensitivity, as well as pain-related gait in a partial sciatic nerve ligation animal model in Chapter $6(R Q 5)$. 


\subsection{References}

1. International Association for the Study of Pain (Retrieved 9 June 2016).

2. Scholz J, Finnerup NB, Attal N, Aziz Q, Baron R, Bennett MI, et al. The IASP classification of chronic pain for ICD-11: chronic neuropathic pain. Pain. 2019;160(1):53-9.

3. Costigan M, Scholz J, Woolf CJ. Neuropathic pain: a maladaptive response of the nervous system to damage. Annu Rev Neurosci. 2009;32:1-32.

4. Backonja MM, Galer BS. Pain assessment and evaluation of patients who have neuropathic pain. Neurol Clin. 1998;16(4):775-90.

5. Attal N, Lanteri-Minet M, Laurent B, Fermanian J, Bouhassira D. The specific disease burden of neuropathic pain: results of a French nationwide survey. Pain. 2011;152(12):2836-43.

6. Meyer-Rosberg K, Kvarnstrom A, Kinnman E, Gordh T, Nordfors LO, Kristofferson A. Peripheral neuropathic pain--a multidimensional burden for patients. Eur J Pain. 2001;5(4):379-89.

7. Krames ES. The dorsal root ganglion in chronic pain and as a target for neuromodulation: a review. Neuromodulation. 2015;18(1):24-32; discussion

8. Devor M. Unexplained peculiarities of the dorsal root ganglion. Pain. 1999;Suppl 6:S27-35.

9. Xiao HS, Huang QH, Zhang FX, Bao L, Lu YJ, Guo C, et al. Identification of gene expression profile of dorsal root ganglion in the rat peripheral axotomy model of neuropathic pain. Proc Natl Acad Sci U S A. 2002;99(12):83605.

10. Liu CN, Wall PD, Ben-Dor E, Michaelis M, Amir R, Devor M. Tactile allodynia in the absence of C-fiber activation: altered firing properties of DRG neurons following spinal nerve injury. Pain. 2000;85(3):503-21.

11. Woolf CJ, King AE. Dynamic alterations in the cutaneous mechanoreceptive fields of dorsal horn neurons in the rat spinal cord. J Neurosci. 1990;10(8):2717-26.

12. Ji RR, Kohno T, Moore KA, Woolf CJ. Central sensitization and LTP: do pain and memory share similar mechanisms? Trends Neurosci. 2003;26(12):696-705.

13. Michael GJ, Averill S, Nitkunan A, Rattray M, Bennett DL, Yan Q, et al. Nerve growth factor treatment increases brain-derived neurotrophic factor selectively in TrkA-expressing dorsal root ganglion cells and in their central terminations within the spinal cord. J Neurosci. 1997;17(21):8476-90.

14. Geng SJ, Liao FF, Dang WH, Ding X, Liu XD, Cai J, et al. Contribution of the spinal cord BDNF to the development of neuropathic pain by activation of the NR2B-containing NMDA receptors in rats with spinal nerve ligation. Exp Neurol. 2010;222(2):256-66.

15. Kerr BJ, Bradbury EJ, Bennett DL, Trivedi PM, Dassan P, French J, et al. Brain-derived neurotrophic factor modulates nociceptive sensory inputs and NMDA-evoked responses in the rat spinal cord. J Neurosci. 1999;19(12):5138-48.

16. Millan MJ. Descending control of pain. Prog Neurobiol. 2002;66(6):355-474.

17. Attal N, Cruccu G, Baron R, Haanpaa M, Hansson P, Jensen TS, et al. EFNS guidelines on the pharmacological treatment of neuropathic pain: 2010 revision. Eur J Neurol. 2010;17(9):1113-e88.

18. Attal N, Cruccu G, Haanpaa M, Hansson P, Jensen TS, Nurmikko T, et al. EFNS guidelines on pharmacological treatment of neuropathic pain. Eur J Neurol. 2006;13(11):1153-69.

19. Dworkin RH, O'Connor AB, Backonja M, Farrar JT, Finnerup NB, Jensen TS, et al. Pharmacologic management of neuropathic pain: evidence-based recommendations. Pain. 2007;132(3):237-51.

20. Moulin D, Boulanger A, Clark AJ, Clarke H, Dao T, Finley GA, et al. Pharmacological management of chronic neuropathic pain: revised consensus statement from the Canadian Pain Society. Pain Res Manag. 2014;19(6):32835 .

21. Tan T, Barry P, Reken S, Baker M, Guideline Development G. Pharmacological management of neuropathic pain in non-specialist settings: summary of NICE guidance. BMJ. 2010;340:c1079.

22. Colloca L, Ludman T, Bouhassira D, Baron R, Dickenson AH, Yarnitsky D, et al. Neuropathic pain. Nat Rev Dis Primers. 2017;3:17002.

23. Finnerup NB, Attal N, Haroutounian S, McNicol E, Baron R, Dworkin RH, et al. Pharmacotherapy for neuropathic pain in adults: a systematic review and meta-analysis. Lancet Neurol. 2015;14(2):162-73.

24. Scottish Medicines Consortium advice to NHS Scotland (2009). Briefing note. Pregabalin (Lyrica). Report No. 18.

25. Chua NH, Vissers KC, Sluijter ME. Pulsed radiofrequency treatment in interventional pain management: mechanisms and potential indications-a review. Acta Neurochir (Wien). 2011;153(4):763-71. 
26. Sluijter ME, Imani F. Evolution and mode of action of pulsed radiofrequency. Anesth Pain Med. 2013;2(4):139-41.

27. van Kleef M, Liem L, Lousberg R, Barendse G, Kessels F, Sluijter M. Radiofrequency lesion adjacent to the dorsal root ganglion for cervicobrachial pain: a prospective double blind randomized study. Neurosurgery. 1996;38(6):1127-31; discussion 31-2.

28. Van Boxem K, Huntoon M, Van Zundert J, Patijn J, van Kleef M, Joosten EA. Pulsed radiofrequency: a review of the basic science as applied to the pathophysiology of radicular pain: a call for clinical translation. Reg Anesth Pain Med. 2014;39(2):149-59.

29. Sluijter ME. Pulsed radiofrequency. Anesthesiology. 2005;103(6):1313; author reply -4.

30. Cosman ER, Jr., Cosman ER, Sr. Electric and thermal field effects in tissue around radiofrequency electrodes. Pain Med. 2005;6(6):405-24.

31. Hamann W, Abou-Sherif S, Thompson S, Hall S. Pulsed radiofrequency applied to dorsal root ganglia causes a selective increase in ATF3 in small neurons. Eur J Pain. 2006;10(2):171-6.

32. Van Zundert J, de Louw AJ, Joosten EA, Kessels AG, Honig W, Dederen PJ, et al. Pulsed and continuous radiofrequency current adjacent to the cervical dorsal root ganglion of the rat induces late cellular activity in the dorsal horn. Anesthesiology. 2005;102(1):125-31.

33. Yang $\mathrm{CH}$, Chen $\mathrm{KH}$, Huang HW, Sheen-Chen SM, Lin CR. Pulsed radiofrequency treatment attenuates increases in spinal excitatory amino acid release in rats with adjuvant-induced mechanical allodynia. Neuroreport. 2013;24(8):431-6.

34. Cho HK, Cho YW, Kim EH, Sluijter ME, Hwang SJ, Ahn SH. Changes in pain behavior and glial activation in the spinal dorsal horn after pulsed radiofrequency current administration to the dorsal root ganglion in a rat model of lumbar disc herniation: laboratory investigation. J Neurosurg Spine. 2013;19(2):256-63.

35. Park HW, Ahn SH, Son JY, Kim SJ, Hwang SJ, Cho YW, et al. Pulsed radiofrequency application reduced mechanical hypersensitivity and microglial expression in neuropathic pain model. Pain Med. 2012;13(9):1227-34.

36. Podhajsky RJ, Sekiguchi Y, Kikuchi S, Myers RR. The histologic effects of pulsed and continuous radiofrequency lesions at 42 degrees $C$ to rat dorsal root ganglion and sciatic nerve. Spine (Phila Pa 1976). 2005;30(9):1008-13.

37. Erdine S, Yucel A, Cimen A, Aydin S, Sav A, Bilir A. Effects of pulsed versus conventional radiofrequency current on rabbit dorsal root ganglion morphology. Eur J Pain. 2005;9(3):251-6.

38. Van Zundert J, Patijn J, Kessels A, Lame I, van Suijlekom H, van Kleef M. Pulsed radiofrequency adjacent to the cervical dorsal root ganglion in chronic cervical radicular pain: a double blind sham controlled randomized clinical trial. Pain. 2007;127(1-2):173-82.

39. Teixeira A, Grandinson M, Sluijter ME. Pulsed radiofrequency for radicular pain due to a herniated intervertebral disc--an initial report. Pain Pract. 2005;5(2):111-5.

40. Van Boxem K, de Meij N, Kessels A, Van Kleef M, Van Zundert J. Pulsed radiofrequency for chronic intractable lumbosacral radicular pain: a six-month cohort study. Pain Med. 2015;16(6):1155-62.

41. Van Boxem K, van Bilsen J, de Meij N, Herrler A, Kessels F, Van Zundert J, et al. Pulsed radiofrequency treatment adjacent to the lumbar dorsal root ganglion for the management of lumbosacral radicular syndrome: $a$ clinical audit. Pain Med. 2011;12(9):1322-30.

42. Aksu R, Ugur F, Bicer C, Menku A, Guler G, Madenoglu H, et al. The efficiency of pulsed radiofrequency application on $L 5$ and 16 dorsal roots in rabbits developing neuropathic pain. Reg Anesth Pain Med. 2010;35(1):115.

43. Chen KH, Yang CH, Juang SE, Huang HW, Cheng JK, Sheen-Chen SM, et al. Pulsed radiofrequency reduced complete Freund's adjuvant-induced mechanical hyperalgesia via the spinal c-Jun N-terminal kinase pathway. Cell Mol Neurobiol. 2014;34(2):195-203.

44. Erdine S, Bilir A, Cosman ER, Cosman ER, Jr. Ultrastructural changes in axons following exposure to pulsed radiofrequency fields. Pain Pract. 2009;9(6):407-17.

45. Hagiwara S, Iwasaka H, Takeshima N, Noguchi T. Mechanisms of analgesic action of pulsed radiofrequency on adjuvant-induced pain in the rat: roles of descending adrenergic and serotonergic systems. Eur $\mathrm{J}$ Pain. 2009;13(3):249-52.

46. Laboureyras E, Rivat C, Cahana A, Richebe P. Pulsed radiofrequency enhances morphine analgesia in neuropathic rats. Neuroreport. 2012;23(9):535-9.

47. Ozsoylar O, Akcali D, Cizmeci P, Babacan A, Cahana A, Bolay H. Percutaneous pulsed radiofrequency reduces mechanical allodynia in a neuropathic pain model. Anesth Analg. 2008;107(4):1406-11. 
48. Perret DM, Kim DS, Li KW, Sinavsky K, Newcomb RL, Miller JM, et al. Application of pulsed radiofrequency currents to rat dorsal root ganglia modulates nerve injury-induced tactile allodynia. Anesth Analg. 2011;113(3):6106.

49. Wu B, Ni J, Zhang C, Fu P, Yue J, Yang L. Changes in spinal cord met-enkephalin levels and mechanical threshold values of pain after pulsed radio frequency in a spared nerve injury rat model. Neurol Res. 2012;34(4):40814.

50. Melzack R, Wall PD. Pain mechanisms: a new theory. Science. 1965;150(3699):971-9.

51. Noordenbos. Pain. Elsevier, Amsterdam. 1959.

52. Janssen SP, Gerard S, Raijmakers ME, Truin M, Van Kleef M, Joosten EA. Decreased intracellular GABA levels contribute to spinal cord stimulation-induced analgesia in rats suffering from painful peripheral neuropathy: the role of KCC2 and GABA(A) receptor-mediated inhibition. Neurochem Int. 2012;60(1):21-30.

53. Linderoth B, Stiller CO, Gunasekera L, O'Connor WT, Ungerstedt U, Brodin E. Gamma-aminobutyric acid is released in the dorsal horn by electrical spinal cord stimulation: an in vivo microdialysis study in the rat. Neurosurgery. 1994;34(3):484-8; discussion 8-9.

54. Cui JG, O'Connor WT, Ungerstedt U, Linderoth B, Meyerson BA. Spinal cord stimulation attenuates augmented dorsal horn release of excitatory amino acids in mononeuropathy via a GABAergic mechanism. Pain. 1997;73(1):87-95.

55. Stiller CO, Cui JG, O'Connor WT, Brodin E, Meyerson BA, Linderoth B. Release of gamma-aminobutyric acid in the dorsal horn and suppression of tactile allodynia by spinal cord stimulation in mononeuropathic rats. Neurosurgery. 1996;39(2):367-74; discussion 74-5.

56. Slangen R, Schaper NC, Faber CG, Joosten EA, Dirksen CD, van Dongen RT, et al. Spinal cord stimulation and pain relief in painful diabetic peripheral neuropathy: a prospective two-center randomized controlled trial. Diabetes Care. 2014;37(11):3016-24.

57. de Vos CC, Meier K, Zaalberg PB, Nijhuis HJ, Duyvendak W, Vesper J, et al. Spinal cord stimulation in patients with painful diabetic neuropathy: a multicentre randomized clinical trial. Pain. 2014;155(11):2426-31.

58. Deer TR, Levy RM, Kramer J, Poree L, Amirdelfan K, Grigsby E, et al. Dorsal root ganglion stimulation yielded higher treatment success rate for complex regional pain syndrome and causalgia at 3 and 12 months: a randomized comparative trial. Pain. 2017;158(4):669-81.

59. North RB, Kumar K, Wallace MS, Henderson JM, Shipley J, Hernandez J, et al. Spinal cord stimulation versus re-operation in patients with failed back surgery syndrome: an international multicenter randomized controlled trial (EVIDENCE study). Neuromodulation. 2011;14(4):330-5; discussion 5-6.

60. Kumar K, Taylor RS, Jacques L, Eldabe S, Meglio M, Molet J, et al. The effects of spinal cord stimulation in neuropathic pain are sustained: a 24-month follow-up of the prospective randomized controlled multicenter trial of the effectiveness of spinal cord stimulation. Neurosurgery. 2008;63(4):762-70; discussion 70.

61. Kramer J, Liem L, Russo M, Smet I, Van Buyten JP, Huygen F. Lack of body positional effects on paresthesias when stimulating the dorsal root ganglion (DRG) in the treatment of chronic pain. Neuromodulation. 2015;18(1):50-7; discussion 7.

62. Kriek N, Groeneweg JG, Stronks DL, de Ridder D, Huygen FJ. Preferred frequencies and waveforms for spinal cord stimulation in patients with complex regional pain syndrome: A multicentre, double-blind, randomized and placebo-controlled crossover trial. Eur J Pain. 2017;21(3):507-19.

63. Perruchoud C, Eldabe S, Batterham AM, Madzinga G, Brookes M, Durrer A, et al. Analgesic efficacy of high-frequency spinal cord stimulation: a randomized double-blind placebo-controlled study. Neuromodulation. 2013;16(4):363-9; discussion 9.

64. Schu S, Slotty PJ, Bara G, von Knop M, Edgar D, Vesper J. A prospective, randomised, double-blind, placebo-controlled study to examine the effectiveness of burst spinal cord stimulation patterns for the treatment of failed back surgery syndrome. Neuromodulation. 2014;17(5):443-50.

65. Al-Kaisy A, Palmisani S, Pang D, Sanderson K, Wesley S, Tan Y, et al. Prospective, Randomized, ShamControl, Double Blind, Crossover Trial of Subthreshold Spinal Cord Stimulation at Various Kilohertz Frequencies in Subjects Suffering From Failed Back Surgery Syndrome (SCS Frequency Study). Neuromodulation. 2018;21(5):45765.

66. Kapural L, Yu C, Doust MW, Gliner BE, Vallejo R, Sitzman BT, et al. Novel 10-kHz High-frequency Therapy (HF10 Therapy) Is Superior to Traditional Low-frequency Spinal Cord Stimulation for the Treatment of Chronic Back and Leg Pain: The SENZA-RCT Randomized Controlled Trial. Anesthesiology. 2015;123(4):851-60. 
67. Miller JP, Eldabe S, Buchser E, Johanek LM, Guan Y, Linderoth B. Parameters of Spinal Cord Stimulation and Their Role in Electrical Charge Delivery: A Review. Neuromodulation. 2016;19(4):373-84.

68. Kilgore KL, Bhadra N. Reversible nerve conduction block using kilohertz frequency alternating current. Neuromodulation. 2014;17(3):242-54; discussion 54-5.

69. Lempka SF, McIntyre CC, Kilgore KL, Machado AG. Computational analysis of kilohertz frequency spinal cord stimulation for chronic pain management. Anesthesiology. 2015;122(6):1362-76.

70. Van Buyten JP, Al-Kaisy A, Smet I, Palmisani S, Smith T. High-frequency spinal cord stimulation for the treatment of chronic back pain patients: results of a prospective multicenter European clinical study. Neuromodulation. 2013;16(1):59-65; discussion -6.

71. Al-Kaisy A, Van Buyten JP, Smet I, Palmisani S, Pang D, Smith T. Sustained effectiveness of $10 \mathrm{kHz}$ highfrequency spinal cord stimulation for patients with chronic, low back pain: 24-month results of a prospective multicenter study. Pain Med. 2014;15(3):347-54.

72. Thomson SJ, Tavakkolizadeh M, Love-Jones S, Patel NK, Gu JW, Bains A, et al. Effects of Rate on Analgesia in Kilohertz Frequency Spinal Cord Stimulation: Results of the PROCO Randomized Controlled Trial. Neuromodulation. 2018;21(1):67-76.

73. Kapural L, Harandi S. Long-term efficacy of 1-1.2 kHz subthreshold spinal cord stimulation following failed traditional spinal cord stimulation: a retrospective case series. Reg Anesth Pain Med. 2019;44(1):107-10.

74. Al-Kaisy A, Palmisani S, Smith T, Harris S, Pang D. The use of 10-kilohertz spinal cord stimulation in a cohort of patients with chronic neuropathic limb pain refractory to medical management. Neuromodulation. 2015;18(1):18-23; discussion

75. De Ridder D, Vanneste S, Plazier M, van der Loo E, Menovsky T. Burst spinal cord stimulation: toward paresthesia-free pain suppression. Neurosurgery. 2010;66(5):986-90.

76. De Ridder D, Plazier M, Kamerling N, Menovsky T, Vanneste S. Burst spinal cord stimulation for limb and back pain. World Neurosurg. 2013;80(5):642-9 e1.

77. De Ridder D, Lenders MW, De Vos CC, Dijkstra-Scholten C, Wolters R, Vancamp T, et al. A 2-center comparative study on tonic versus burst spinal cord stimulation: amount of responders and amount of pain suppression. Clin J Pain. 2015;31(5):433-7.

78. Liem L, Russo M, Huygen FJ, Van Buyten JP, Smet I, Verrills P, et al. A multicenter, prospective trial to assess the safety and performance of the spinal modulation dorsal root ganglion neurostimulator system in the treatment of chronic pain. Neuromodulation. 2013;16(5):471-82; discussion 82.

79. Huygen F, Liem L, Cusack W, Kramer J. Stimulation of the L2-L3 Dorsal Root Ganglia Induces Effective Pain Relief in the Low Back. Pain Pract. 2018;18(2):205-13.

80. Bayman EO, Brennan TJ. Incidence and severity of chronic pain at 3 and 6 months after thoracotomy: meta-analysis. J Pain. 2014;15(9):887-97.

81. Eldabe S, Burger K, Moser H, Klase D, Schu S, Wahlstedt A, et al. Dorsal Root Ganglion (DRG) Stimulation in the Treatment of Phantom Limb Pain (PLP). Neuromodulation. 2015;18(7):610-6; discussion 6-7.

82. Eldabe S, Espinet A, Wahlstedt A, Kang P, Liem L, Patel NK, et al. Retrospective Case Series on the Treatment of Painful Diabetic Peripheral Neuropathy With Dorsal Root Ganglion Stimulation. Neuromodulation. 2018.

83. Levy RM, Mekhail N, Kramer J, Poree L, Amirdelfan K, Grigsby E, et al. Therapy Habituation at 12 Months: Spinal Cord Stimulation Versus Dorsal Root Ganglion Stimulation for Complex Regional Pain Syndrome Type I and II. J Pain. 2019.

84. Deer TR, Levy RM, Kramer J, Poree L, Amirdelfan K, Grigsby E, et al. Comparison of Paresthesia Coverage of Patient's Pain: Dorsal Root Ganglion vs. Spinal Cord Stimulation. An ACCURATE Study Sub-Analysis. Neuromodulation. 2019.

85. Mekhail N, Deer TR, Kramer J, Poree L, Amirdelfan K, Grigsby E, et al. Paresthesia-Free Dorsal Root Ganglion Stimulation: An ACCURATE Study Sub-Analysis. Neuromodulation. 2019.

86. Graham RD, Bruns TM, Duan B, Lempka SF. Dorsal root ganglion stimulation for chronic pain modulates Abeta-fiber activity but not C-fiber activity: A computational modeling study. Clin Neurophysiol. 2019;130(6):94151.

87. Koetsier E, Franken G, Debets J, Heijmans L, van Kuijk SMJ, Linderoth B, et al. Mechanism of dorsal root ganglion stimulation for pain relief in painful diabetic polyneuropathy is not dependent on GABA release in the dorsal horn of the spinal cord. CNS Neurosci Ther. 2019. 
88. Koopmeiners AS, Mueller S, Kramer J, Hogan QH. Effect of electrical field stimulation on dorsal root ganglion neuronal function. Neuromodulation. 2013;16(4):304-11; discussion 10-1.

89. Kent AR, Min X, Hogan QH, Kramer JM. Mechanisms of Dorsal Root Ganglion Stimulation in Pain Suppression: A Computational Modeling Analysis. Neuromodulation. 2018;21(3):234-46.

90. Du X, Hao H, Yang Y, Huang S, Wang C, Gigout S, et al. Local GABAergic signaling within sensory ganglia controls peripheral nociceptive transmission. J Clin Invest. 2017;127(5):1741-56.

91. Pawela CP, Kramer JM, Hogan QH. Dorsal root ganglion stimulation attenuates the BOLD signal response to noxious sensory input in specific brain regions: Insights into a possible mechanism for analgesia. Neuroimage. 2017;147:10-8. 


\section{CHAPTER}

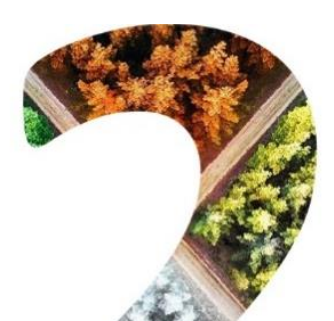

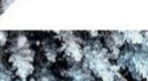

Spinal Cord Stimulation in Chronic

Neuropathic Pain: Mechanisms of

Action, New Locations, New Paradigms

E.A. Joosten \& G. Franken

Published. PAIN 2020; 161 Suppl 1:S104-S13. 


\subsection{Abstract}

Spinal cord stimulation (SCS) is usually a minimally invasive therapy and has proven to be a valuable last-resort treatment option for a wide variety of refractory (peripheral) neuropathic pain disorders. Conventional or Tonic SCS (with an amplitude above sensory threshold) is characterized by the presence of paresthesias that overlap the painful area, and provides pain attenuation for some patients. The analgesic effect of Tonic SCS is thought to be mainly mediated via segmental spinal mechanisms by activation of dorsal column $A \beta$ fibers, but also supraspinal mechanisms and cortical processing are involved in the pain relieving effect of Tonic SCS. An important limitation of Tonic SCS is that only $50-70 \%$ of patients with refractory neuropathic pain achieve pain reductions of $\geq 50 \%$, and the average pain reduction is restricted to approximately $50-60 \%$. With the introduction of new anatomical locations for stimulation, like dorsal root ganglion stimulation (DRGS), and the addition of new sub-sensory stimulation paradigms like high frequency (HF) SCS and Burst SCS, options for improvement are available. The mechanisms of action of these new SCS paradigms are incompletely understood, but it seems that specific stimulation of cortical brain areas is involved and this accounts for modulation of motivational, affective, cognitive and emotional components of pain. Future mechanistic and clinical studies are needed to further optimize the efficacy of SCS and develop new (personalized) stimulation strategies for patients with refractory neuropathic pain.

Keywords: Spinal cord stimulation, Dorsal root ganglion stimulation, Burst SCS, High frequency SCS, Neuropathic pain, GABA 


\subsection{Introduction}

Neuropathic pain is a complex, heterogeneous disorder that affects approximately $8 \%$ of the total adult human population and comes with significant burden for both the patient and healthcare system [1]. The international association for the study of pain (IASP) defines neuropathic pain as "pain caused by a lesion or disease of the somatosensory nervous system", and classifies chronic neuropathic pain as a disease under International Classification of Diseases 11th Revision (ICD-11) [2]. Despite the development and use of many pharmacological drugs and guidelines for the treatment of chronic neuropathic pain over the years [3], a substantial amount of neuropathic pain patients remain under- or untreated, with less than $50 \%$ of patients responding to pharmacological treatment [4]. The development of novel, last-resort interventional treatment therapies is crucial to also relief pain in these refractory patients.

Over the years, spinal cord stimulation (SCS) has proven to be a valuable last-resort treatment option (approximately 50\% pain reduction in $50-70 \%$ of patients) for a wide variety of refractory pain disorders, such as painful diabetic peripheral neuropathy (PDPN) [5, 6], complex regional pain syndrome (CRPS) $[7,8]$ and failed back surgery syndrome (FBSS) $[9$, 10]. The mechanism underlying Tonic SCS (see section 2.3) is partly understood, and evidence has been provided for a mechanism of action via both spinal (section 2.3.1) as well as supraspinal levels (section 2.3.2). Recently, new physiological targets for stimulation as well as novel SCS paradigms were introduced in order to bridge the gap between currently achieved pain relief (as obtained with Tonic SCS) and the desired pain relief. Literature on the effect of stimulation at new anatomical locations, such as dorsal root ganglion stimulation (DRGS) (see section 2.4), and the use of new subsensory SCS paradigms like high frequency (HF) SCS (see section 2.5.2) and Burst SCS (see section 2.5.3) are discussed. This review ends with concluding remarks and future directions for research. 


\subsection{Tonic SCS: Mechanisms of action}

\subsubsection{Tonic SCS and spinal segmental mechanisms}

Experimental studies on the effect of SCS have predominantly been performed in rodent models including the partial sciatic nerve ligation model (PSNL) (for review see Smits et al., 2013 [11]). Electrodes are carefully inserted, either transcutaneous or via laminectomy, in the epidural space on top of the dura mater surrounding the spinal cord. Then, electrical pulses are administered to the dorsal columns of the spinal cord via an implantable pulse generator or an external stimulation device. Tonic SCS settings vary within a range of 30-80 $\mathrm{Hz}, 100-500 \mu \mathrm{s}$ pulse width, and an amplitude above sensory threshold [11-14].

The concept of Tonic SCS emerged as a direct spin-off from the Gate Control Theory [15]. Based on this Gate Control Theory, it was postulated that antidromic stimulation of the non-nociceptive $A \beta$-fibers in the dorsal columns could close a 'spinal gate', located in the dorsal horn of the spinal cord [16]. Meanwhile, orthodromic stimulation of the A $\beta$-fibers in the dorsal columns also caused paresthesias (i.e., abnormal tingling sensation) in the area innervated by the stimulated fibers [17] (Figure 1). Nowadays, during implantation of the SCS lead the physician makes sure these paresthesias overlap the painful area $[17,18]$. Closing of the 'spinal gate' is mediated by inhibitory interneurons located in the upper laminae of the dorsal horn. In line with the Gate Control Theory these inhibitory interneurons, when antidromically activated by Tonic SCS, modulate the nociceptive signal through the release of gamma-amino-butyric acid (GABA). Indeed, experimental research has demonstrated that Tonic SCS decreases intracellular GABA immunoreactivity in the dorsal horn of chronic neuropathic rats [19]. At the same time, extracellular GABA levels in the spinal dorsal horn increase when applying Tonic SCS in chronic neuropathic rats [20-22]. Thus, enhanced GABA release in the spinal dorsal horn seems to be a vital aspect of the mechanisms underlying Tonic SCS. The mechanism underlying interference with nociception at the spinal cord level using Tonic SCS was further elucidated by the administration of pharmacological agents. Local intrathecal application of a $\mathrm{GABA}_{\mathrm{B}}$-receptor antagonist in the dorsal horn transiently abolished the stimulation-induced analgesic effect in neuropathic rats, and rats not receiving 
adequate reductions in tactile allodynia with Tonic SCS (non-responders) were turned into responders by administration of the GABAB-receptor agonist baclofen [23]. The aforementioned preclinical findings were successfully translated to the clinic, where some neuropathic pain patients not responding to Tonic SCS were turned into responders with additional intrathecal administration of low (sub-effective) doses of baclofen [24-26]. Hence, the presynaptic $\mathrm{GABA}_{B}$-mediated inhibition of the communication between nociceptive afferents and the second order neurons in the spinal dorsal horn is important in the mechanism underlying Tonic SCS. Nevertheless, also postsynaptic GABAergic modulation via $\mathrm{GABA}_{A}$ receptors in conjunction with $\mathrm{K}^{+} / \mathrm{Cl}^{-}$cotransporter $2(\mathrm{KCC} 2)$ expression is involved in neuropathic pain [27] and in the mechanism underlying Tonic SCS [19, 23, 28].

A decreased GABA-release as noted in animal models of neuropathic pain results in further enhanced and uncontrolled glutamate release of the nociceptive afferents, which in turn activates and opens the N-methyl-D-aspartate (NMDA) receptor due to removal of the $\mathrm{Mg}^{2+}$ block. Enhanced $\mathrm{Ca}^{2+}$ influx via the NMDA-receptor then leads to central sensitization, which is a process fundamental to neuropathic pain [29]. From this, it was suggested that interference with the process of central sensitization via antagonism of the NMDA receptor might attenuate chronic neuropathic pain, a process that may also be involved in the antidromic mechanism underlying Tonic SCS. Indeed, a combined treatment of Tonic SCS and the intrathecal application of a sub-effective dose of ketamine (a NMDA-antagonist replacing the $\mathrm{Mg}^{2+}$ block) has been shown to convert SCS non-responder into responders in a rat model of chronic neuropathic pain [30]. It needs to be stressed that these experimental findings have not yet been implemented and/or confirmed in clinical studies. Importantly, intrathecal administration of ketamine was shown to result in severe histological abnormalities, including central chromatolysis, nerve cell shrinkage, neuronophagia, microglial upregulation, and gliosis in a patient suffering from chronic intractable neuropathic pain [31]. Although it is very well possible that sub-effective doses of ketamine can in fact be safely used in a clinical setting, more research is needed as to determine safe intrathecal administration dosages. 


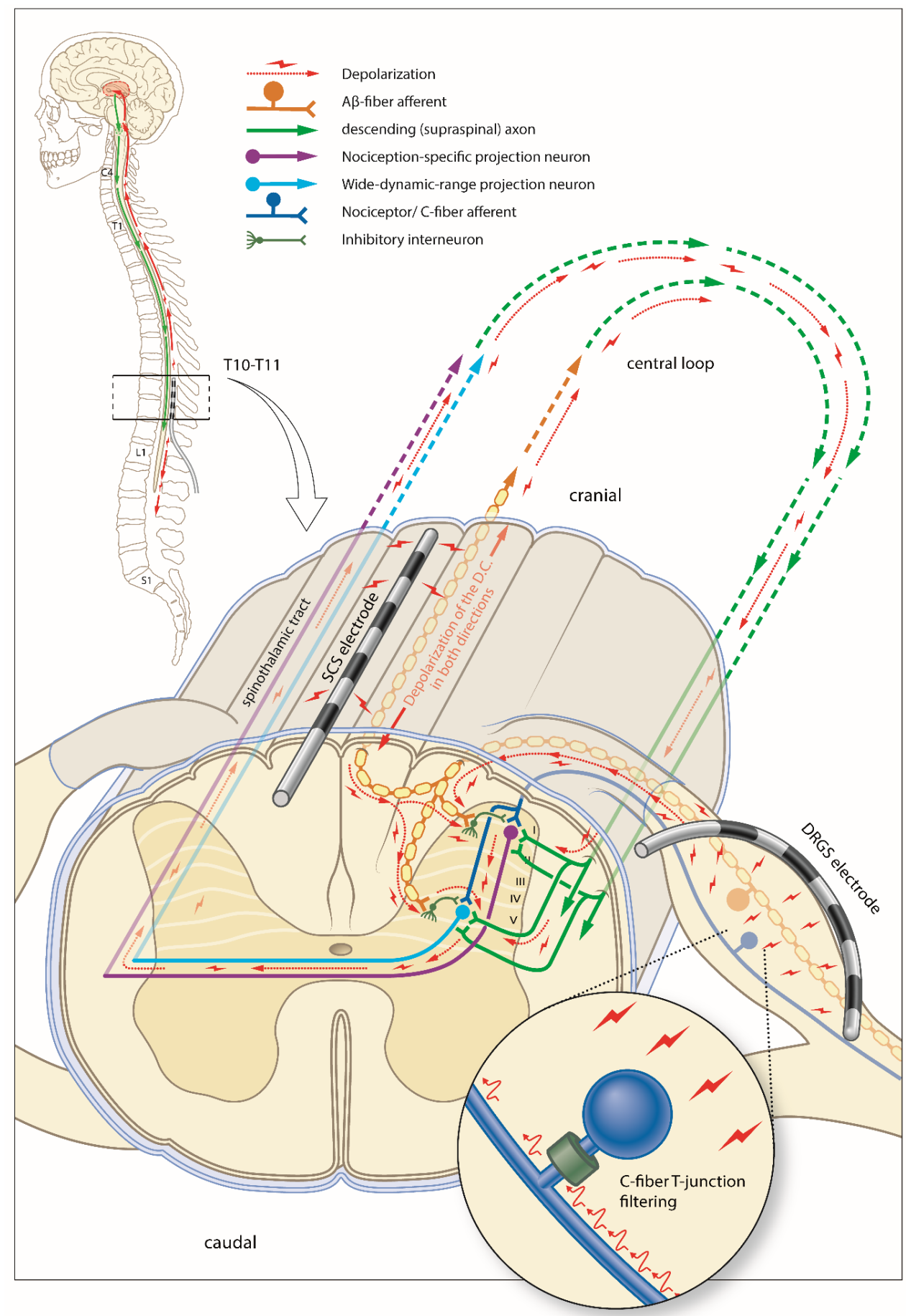


Figure 1. The spinal nociceptive network and mechanisms of action of SCS of the dorsal columns and DRGS. The spinal cord dorsal horn contains two types of second-order projection neurons: the nociceptive specific (NS) projection neurons located in lamina I, and the wide-dynamic range (WDR) projection neurons located in the deeper laminae. These projection neurons receive input from nociceptive afferents, but also from thickly myelinated, touch-affiliated, A $\beta$-fiber afferents. SCS (electrode placed on top of the dorsal columns) is thought to depolarize the touch-affiliated $A \beta$-fibers and this can occur in both the antidromic and orthodromic direction. Antidromically, SCS can activate GABAergic inhibitory interneurons located in the dorsal horn. Consequently, these inhibitory interneurons release GABA, which, after binding to its GABA receptor (either to $G A B A_{B}$ or $G A B A_{A}$ pre- or post-synaptically), inhibits the incoming signals from nociceptors and thereby closes the 'spinal gate'. Additionally, SCS can also interfere with further processing of the nociceptive signal via the spinothalamic tract, thereby modulating supraspinal brain centers such as the thalamus, somatosensory cortex, cingulate cortex, and insula. Orthodromically, SCS can also depolarize A $\beta$-fibers in the cranial direction, thereby further modulating supraspinal centers like the cuneate nucleus or gracile nucleus. After supraspinal integration of the signal, a descending feedback loop of both serotonergic and noradrenergic projections to the dorsal spinal horn further modulates and controls the 'spinal gate'. DRGS (electrode placed on top of the DRG) might engage mechanisms dependent on stimulation of non-nociceptive A $\beta$-fibers (as occurs in SCS) as well as stimulation of nociceptive Cfibers in the DRG. Recent studies suggest that DRGS may induce a conduction block via the C-type T-junction located in the DRG itself. This T-junction can act as a low-pass filter for action potentials (nociceptive signals) travelling from the periphery to the spinal cord.

The main goal of Tonic SCS in the treatment of (experimental) chronic neuropathic pain is to stimulate the thickly myelinated $A \beta$-fibers in the dorsal columns. It can, however, not be excluded that also incoming dorsal root fibers, including C- and A $\delta$-fibers, are directly stimulated through the relatively large-sized experimental electrodes as used in rodent studies [11]. This possible involvement of dorsal root fibers and the dorsal root as the site of action is further substantiated by electrophysiological analysis where not only stimulation of the dorsal column but also stimulation of the dorsal root attenuated dorsal horn neuronal hyperexcitability in nerve-injured rats [32].

Although Tonic SCS and its spinal mechanisms are partly uncovered, recent studies indicate that much more complicated interactions and cell types are involved. Tonic SCS causes long-term depression of excitatory synaptic transmission in the superficial dorsal horn (lamina II), and this depression is blocked by antagonists of cannabinoid receptor type 1 (CB1) 
[33]. Furthermore, the intrathecal application of AM251, a CB1 receptor antagonist, was able to block SCS-mediated reversal of mechanical hypersensitivity in rats [34]. The CB1 receptor is located on microglial cells [34], which indicates that the endocannabinoid system, and in particular the CB1 receptor, plays a pivotal role in the reversal of hyperalgesia induced by SCS and links the mechanism underlying Tonic SCS analgesia to glia mediated control of nociception [35].

\subsubsection{Tonic SCS and suprasegmental mechanisms}

Once activated, supraspinal cell regions are known to modulate the incoming nociceptive signals at the spinal level via descending fiber projections. Brainstem nuclei such as the locus coeruleus (LC) and the nucleus raphe magnus (NRM), but also the rostral ventromedial medulla (RVM), are activated by Tonic SCS and in turn modulate the spinal nociceptive signal (Figure 1). The descending projections release a variety of neurotransmitters including serotonin $(5-\mathrm{HT})$, which exerts an inhibitory effect (based on the receptor involved) on the incoming nociceptive fibers [36-40], and this maintains long-term neuropathic pain [41]. Further detailed research on the spinal 5-HT receptors that contribute to the pain relieving effects of Tonic SCS in chronic neuropathic rats was performed, and with use of intrathecal application of antagonists and agonists for the various serotonin receptors it was shown that the activation of the 5HT-3 receptor appears to operate via spinal GABAergic interneurons [42].

First evidence for a role of suprasegmental mechanisms underlying Tonic SCS was presented by El-Khoury and colleagues [43], who demonstrated that Tonic SCS of the dorsal column nuclei reduces allodynia and hypersensitivity in an experimental model of chronic neuropathic pain, even after dorsal column transection below these nuclei. From this, it was suggested that the observed inhibition in terms of allodynia and hypersensitivity responses can be attributed to the activation of brainstem pain-modulating centers via rostral projections of the dorsal column nuclei.

That Tonic SCS can also modulate activation patterns in brain areas at subcortical and cortical levels has been shown in a rodent model of chronic neuropathic pain $[44,45]$. 
How Tonic SCS alters cortical processing has also been shown by clinical studies using imaging approaches such as functional magnetic resonance imaging (fMRI), positron-emission tomography (PET), single-photon emission computed tomography (SPECT), and 133-Xe inhalation (reviewed in Bentley et al., 2016 [46]). These cortical changes during Tonic SCS may represent direct effects from dorsal column stimulation or inhibition of nociceptive signals arising from the periphery, or they may reflect complex modulatory effects on somatosensory and affective processing. Early clinical fMRI-work on the supraspinal effects of Tonic SCS has demonstrated modulation of brain regions associated with the lateral spinothalamic tract (I-STT) [47, 48]. The I-STT is responsible for the transmission of pain aspects such as the intensity and location of the painful stimulus. This I-STT pathway projects from the dorsal horn, via the thalamus, to cortical areas such as the somatosensory cortex [49]. An fMRI study performed in eight patients receiving Tonic SCS demonstrated that this type of stimulation of the dorsal columns increased blood oxygen level-dependent (BOLD) signals in somatosensory cortices, the sensorimotor cortex and the insula [48]. Furthermore, a more recent $\mathrm{fMRI}$-study with twenty patients who received Tonic SCS as treatment for FBSS, reported deactivation of the bilateral medial thalamus and its connections to the rostral and caudal cingulate cortex, and the insula [50]. In conclusion, over the years, literature on Tonic SCS has provided evidence for a mechanism of action via both spinal as well as supraspinal levels.

\subsubsection{Tonic SCS and translation of experimental studies}

It should be noted that the majority of preclinical studies still rely on behavioral analysis based on Von Frey paw withdrawal testing, a technique unable to assess supraspinal cognitive-motivational aspects of pain [51]. Although the peripheral nerve injuries as used in experimental animal studies do definitely result in chronic pain, the rather exclusive use of Von Frey testing is much more related to assessment of nociception instead of pain [52]. This may underlie the limited translation of experimental findings to the clinic $[51,53]$. Recently, an operant testing method was introduced which assesses cognitive and motivational aspects of pain in rodents: the Mechanical Conflict-Avoidance System (MCAS) [54]. With use of MCAS, Tonic SCS was shown to affect also the cognitive-motivational aspects of the 
presumed pain in chronic neuropathic rats [45]. This indicates that Tonic SCS, in addition to local spinal modulation, also recruits supraspinal brain areas, a finding further substantiated by $\mathrm{fMRI}$-analysis of brain areas including the anterior cingulate cortex [44]. These findings make clear that operant behavioral testing should be considered when analyzing the analgesic effects of SCS in chronic neuropathic pain, as this is not only likely to increase the translation of experimental findings to the clinic, but will also help to better understand the underlying mechanisms of action.

Additionally, also other discrepancies between humans and rodents may impact direct translation of laboratory findings to the clinic. These include the standardized models used (in comparison to heterogeneous clinical populations), the use of motor thresholds (instead of perception thresholds in humans) for determining stimulation amplitude, the size of the electrode in relation to the dorsal columns (typically larger in rodents), differences in dorsal column anatomy, and the thickness of the cerebrospinal fluid (CSF) layer that lies between the SCS lead and dorsal column fibers [11]. Therefore, it is important to always carefully consider these discrepancies when trying to extrapolate preclinical findings to the clinic.

\subsubsection{Tonic SCS: Which dorsal column fibers are stimulated?}

Although both spinal and supraspinal activation is involved in Tonic SCS, it has been demonstrated that Tonic SCS results in greater reductions of mechanical allodynia in the rat when administered at the level where the injured sciatic nerve fibers enter the spinal dorsal horn (=T13), as compared to application at more rostral levels (=T11) [55]. The anatomy of the dorsal column in the rat spinal cord makes the $A \beta$-fibers level localized dorsolateral within the columns at T13 (where injured fibers enter) but rearrange to ventromedial positions at more rostral levels $(\mathrm{T} 11)[55,56]$. The majority of ascending dorsal column $A \beta$-fibers were also found to be lost from the dorsal columns and only $15 \%$ reaches cervical levels [56]. Furthermore, computer-modelling and calculations on the fraction of dorsal column fibers that are actually being stimulated (and depolarized) by Tonic SCS found that this is not likely to exceed $1 \%$ of the most superficially (dorsally) located afferents, as the ability of the SCS 
electrode to depolarize dorsal column fibers decreases to the third power of the distance from the electrode $[57,58]$. As the behavioral findings on pain relief of Tonic SCS in a model of chronic neuropathic pain [55] are in line with the aforementioned anatomical and physiological principles it is concluded that Tonic SCS primarily acts through a segmental, spinal, site of action (Figure 1).

In the context of dorsal column anatomy, it should be stressed that these fiber systems not only include large myelinated $A \beta$-fibers but also contain even larger numbers of unmyelinated fibers in the rat [59] and human [60], something that is often neglected. Because of the importance of the dorsal columns in somatic sensation, and as the origin of these unmyelinated fibers is still not fully understood, it is extremely important to understand where these fibers originate. Although the unmyelinated fibers may belong to various categories including proprioceptive, corticospinal or fibers descending from cells in the nucleus gracilis or cuneate $[59,61]$, unilateral dorsal root transection revealed that a significant fraction of the unmyelinated fibers in the fasciculus gracile ascend, presumably to the nucleus gracilis in the brain stem, and also that a significant number of these fibers branch [62]. Moreover, based on pharmacological intervention studies it is strongly suggested that, at least at cervical levels, a subset of these unmyelinated fibers might be nociceptive and involved in noxious processing [63]. This then may shed a more complicated view on the mechanism underlying Tonic SCS, as not only non-nociceptive A $\beta$-fibers but also nociceptive unmyelinated C-fibers are stimulated. In this context, it is interesting that a detailed protocol for the identification of superficial dorsal horn spinal cord neurons that receive peripheral input and project to the brain was recently presented [64]. This may allow further identification of not only nociceptive specific (NS) cells in the dorsal horn, but also their possible (unmyelinated) ascending projections in the dorsal column.

\subsubsection{Tonic SCS: Limitations}

Despite considerable improvements, there are, however, limitations to the efficacy of Tonic SCS. First, only 50-70\% of patients with PDPN, CRPS or FBSS achieve pain reductions of $\geq 50 \%$ [5-10]. Second, the average pain reduction is restricted to approximately $50-60 \%$ [5- 
10]. Third, Tonic SCS is often unable to satisfactory and specifically stimulate difficult-toreach areas, such as the extremities or the groin. Fourth, placement of the leads on top of the dorsal columns makes this therapy susceptible to postural variations due to changes in distance between stimulation lead and stimulation target, leading to unpleasant paresthesias and/or overstimulation [11]. Last, with Tonic SCS there is significant energy loss to the local environment such as the CSF, before the electrical energy (charge) reaches the spinal cord dorsal columns [14]. It is important to note that recent developments in the field of SCS may result in overcoming these limitations. These developments will be discussed as related to either the use of new locations for stimulation (see section 2.4), and/or the use of new SCS paradigms (see section 2.5).

\subsection{New stimulation location: The dorsal root ganglion}

With DRGS, the leads are transcutaneously implanted in the epidural space on top of the dura mater surrounding the spinal cord, but are then advanced laterally through the intervertebral foramen, in order to place the lead over the DRG of interest. Since the first fully implanted DRGS system in 2013 [65], DRGS has been successfully implemented for a wide variety of neuropathic pain disorders, including, but not limited to, discogenic low back pain [66], CRPS type I and II [67], post-amputation pain [68], and PDPN [69]. Yet, only one randomized clinical trial (RCT) on DRGS has been published to date [67]. This study found DRGS to be non-inferior and superior to Tonic SCS for treating chronic intractable pain of the lower limbs attributed to CRPS type I and II. Additionally, patients receiving DRGS were found to have less therapy habituation as compared to patients treated with Tonic SCS at 9 and 12 months [70]. Also the amount and intensity of paresthesias were found to be less with DRGS over Tonic SCS, and DRGS was found to be more stable in response to changes in body position as compared to Tonic SCS [71]. Lastly, some DRGS patients even achieved paresthesia-free analgesia [72].

Mechanistically, it was initially assumed that DRGS engages the spinal mechanisms dependent on stimulation of non-nociceptive $A \beta$-fibers and GABA release in the dorsal horn of the spinal cord as occurs in Tonic SCS of the dorsal columns. While a recent computational 
study indeed suggested that DRGS may inhibit nociception by activating pain-gating mechanisms in the dorsal horn via repeated activation of large myelinated $(A \beta)$ afferents [73], another study found that the pain relieving effect of DRGS is not likely to be dependent on GABA release in the spinal dorsal horn at the L4-L6 lumbar level [74]. Some experimental studies suggest that instead, DRGS suppresses excitability of neurons with predominantly slow-conducting fibers (C-fibers) $[75,76]$. Because of the unique pseudo-unipolar design of DRG neurons, the DRG is likely to act as an impediment or low-pass filter to electrical impulses traveling from the peripheral nociceptor to the spinal cord in response to electrical stimulation (Figure 1) [75-77]. Interestingly, a recent study by Du et al. found an extensive GABAergic communication network between sensory neuron somata inside the DRG. These authors showed that sensory neurons in the DRG express major proteins required for GABA synthesis and release, and are capable of releasing GABA upon depolarization. From this it was proposed that this GABAergic system in the DRG may act as a second gate, in addition to the aforementioned gate control theory (or first gate), and that DRGS might exert its analgesic action by engaging this second gate [78]. This proposed conduction block at the site of the DRG is consistent with the observation that DRGS attenuates BOLD signals of brain areas that are considered to be part of the pain matrix including the contralateral thalamic nuclei, and cortical S1 and S2 that were increased by noxious hind-limb stimulation in rats [79].

Although promising, the therapeutic efficacy of DRGS should be confirmed and verified in additional large-scale RCTs including different pain etiologies. Future experimental studies are also needed to unravel the underlying mechanisms of DRGS, including the role of a hypothetical second (GABAergic) gate in the DRG itself.

\subsection{The use of new SCS paradigms: High Frequency SCS and Burst SCS}

\subsubsection{Introduction}

Next to novel physiological targets for stimulation, novel SCS paradigms were introduced in order to bridge the gap between currently achieved and desired pain relief. Two prominent examples, HF SCS and Burst SCS, were recently introduced in order to try to 
optimize the efficacy of SCS treatment for chronic neuropathic pain [13, 80-86]. Both HF SCS and Burst SCS are generally applied at stimulation amplitudes below sensory threshold, which means the patient does not experience paresthesias during stimulation. This has offered researchers, for the first time since (Tonic) SCS was introduced in 1967 [16], the opportunity to perform double-blind placebo-controlled clinical studies [87-91].

\subsubsection{High Frequency SCS in neuropathic pain}

HF SCS is generally applied at a frequency above $1000 \mathrm{~Hz}$, up until $10 \mathrm{kHz}$, with a pulse width at approximately $30 \mu \mathrm{s}$ and an amplitude of typically 1-5 mA [92]. Hypotheses about the underlying mechanism of HF SCS vary.

Whereas Tonic SCS and its pain inhibition is accompanied by paresthesias, the subthreshold HF SCS paradigm is paresthesia-free (administered below sensory threshold) and does not activate or change the conduction properties of the dorsal column A $\beta$-fibers [93-95]. Experimental research has shown that the dorsal column nuclei are activated with use of Tonic SCS, while with subthreshold HF SCS the neurons in the gracile nucleus do not show a reduction of evoked responses upon peripheral stimulation in a chronic neuropathic pain model [96]. A hypothetical mechanism for HF SCS and its pain relieving effect was brought forward by Chakravarthy and colleagues, who suggested that the electrical current applied to the spinal cord surface may generate a weak and localized electric field of electrochemical disturbance in the spinal dorsal horn and dorsal root entry zone [14, 95]. Hence, HF SCS in fact desynchronizes the communication between the nociceptive C-fibers, which mainly terminate in the dorsal horn superficial laminae (Lamina 1-3), and the NS neurons (Figure 1). Besides the generation of a weak electrical field in the superficial dorsal horn, the hypotheses about the underlying mechanism of HF SCS also include 1) temporal summation which could play a role, where multiple pulses build on each other to achieve neuronal activation; and 2) a depolarization blockade which might occur and where propagating action potentials are differentially blocked by the high frequency stimulation $[14,94,95,97,98]$. 
Until today, the optimal frequency for HF SCS has not yet been determined, and clinical evidence suggests that different HF SCS frequencies can yield clinically significant pain relief $[81,83,90,92,99]$.

\subsubsection{Burst SCS in neuropathic pain}

The Burst paradigm was introduced in 2010 by de Ridder and colleagues [86]. This Burst waveform consists of five closely spaced monophasic spikes administered at $40 \mathrm{~Hz}$ interburst mode and $500 \mathrm{~Hz}$ intraburst frequency, with a pulse width of $1 \mathrm{~ms}$ and $1 \mathrm{~ms}$ interspike interval, delivered in constant current mode. The cumulative charge of the five 1 ms spikes is balanced during the $5 \mathrm{~ms}$ after the spikes, in a so-called passive recharge phase, which differentiates it from HF SCS and Tonic SCS, in which each pulse is immediately charge balanced after each spike, in a so-called active recharge phase $[84,86,89]$. This Burst pattern was chosen because it supposedly mimics naturally occurring neural bursting patterns in the central nervous system. Indeed, neurons responsible for encoding aspects of nociception from peripheral neurons [100-102] and the thalamus [103-105] have been reported to fire in bursting patterns. Although possible overlap between the original Burst waveform (as proposed and used by De Ridder and colleagues) $[86,89]$ and the neural bursting patterns in the central nervous system (CNS), it is important to note that Burst parameters have not yet been optimized in relation to pain relieving capacity because the parameter space has not been fully explored. For instance, effect differences of active versus passive charge recovery have not been characterized. Beyond charge recovery, many other parameters can be varied: interburst frequency, intraburst frequency, pulse width, shape of pulse, but also the number of pulses. Future research is needed to optimize burst programming as well as to elucidate how the physiological changes produced by different Burst SCS paradigms are reflected in preclinical behavior and in the clinic [106].

Like HF SCS, the Burst paradigm has been reported to produce pain relief without inducing paresthesias in the majority of patients, suggesting that stimulation is not activating dorsal column A $\beta$-fibers $[107,108]$. However, even though stimulation at low amplitude may be subthreshold with respect to neuronal activation, and subperception with respect to the 
patient's experience, large amounts of charge are still delivered to dorsal horn fibers, providing the pulse width and/or frequency are sufficiently large [14]. This could potentially set in motion additional dorsal horn mechanisms that are not activated with suprathreshold Tonic SCS. Yet, the key difference between Tonic SCS and Burst SCS is thought to be located higher up the neuraxis, at supraspinal levels. Clinical evidence suggests that Burst SCS not only stimulates sensorimotor cortex areas via the I-STT (known to be involved in localization and intensity of pain), but also specifically stimulates the medial spinothalamic tract (m-STT), which is known to target limbic brain areas involved in cognitive-motivational and emotional aspects of pain, such as the amygdala, the anterior cingulate cortex, and the insula $[85,89]$. Additionally, it was found that Burst SCS improves pain aspects including the amount of attention patients pay to pain' as well 'changes in pain', as assed by the Pain Vigilance and Awareness Questionaire (PVAQ), to a greater degree than Tonic SCS or placebo stimulation [89]. Interestingly, while Burst SCS resulted in significantly more improvement in terms of limb and back pain than placebo on the Visual Analog Scale (VAS), no significant differences between Burst and Tonic SCS were observed in terms of VAS scores [89]. These findings are further substantiated by the fact that Burst and Tonic SCS do share brain activation patterns of the I-STT as well as descending pain inhibitory pathways $[85,89]$. Combined, these data suggest that both Burst SCS and Tonic SCS are capable of modulating the I-STT, but Burst SCS adds to this by also modulating the m-STT. Modulation of the m-STT may hereby improve the affective component of the pain experience.

In order to further elucidate the mechanism underlying Burst SCS and pain relief, experimental studies are needed. As the majority of experimental studies on the effect of Tonic SCS were performed in sciatic nerve injury models including the PSNL model (see section 2.3.1), it is important to use similar models to adequately compare and correlate findings. As the administration of both bicuculline $\left(G A B A_{A}\right)$ and phaclofen $\left(G A B A_{B}\right)$ receptor antagonists abolishes the pain relieving effect of both Tonic SCS but also Burst SCS in a PSNL rat model of chronic neuropathic pain, it is concluded that Burst SCS, like Tonic SCS, is mediated via spinal GABAergic mechanisms [109]. Since Burst SCS is suggested to modulate structures at a supraspinal levels in a different manner as compared to Tonic SCS [85, 89], it 
is remarkable that the GABAergic mechanisms underlying these different stimulation waveforms, at least at a spinal level, show similarities [109]. On the other hand, with the use of escape latency in the MCAS [54], the cognitive-motivational aspects of Burst SCS were analyzed and compared to Tonic SCS in a rat model of chronic neuropathic pain [45] (see also section 2.3.2). With the MCAS, Burst SCS exit latencies differed significantly from Tonic SCS exit latencies and from this it was concluded that Burst SCS specifically affects, much more than Tonic SCS, supraspinal areas responsible for the processing of cognitive-motivational aspects of pain. These findings were further substantiated with fMRI imaging [44]: fMRI analysis of Burst SCS in chronic neuropathic animals showed specific involvement and activation of limbic brain areas including the Anterior Cingulate Cortex (ACC) as well as the Amygdala and Insula, areas known to be involved in cognitive and emotional aspects of pain. The behavioral and imaging studies on Burst SCS and Tonic SCS in pain relief in a neuropathic animal model strongly suggest that the mechanism underlying Burst SCS significantly differs from that of Tonic, although some overlap in underlying mechanism (e.g. GABA release in dorsal spinal horn) does exist.

The fact that Burst SCS has been shown to result in a delayed wash-in and delayed wash-out analgesic effect in a chronic neuropathic pain model as compared to Tonic SCS [110, 111] might provide some additional clues about the underlying mechanism. As the Burst SCS paradigm mainly activates ascending pathways including the I-STT and m-STT (Figure 1), it is possible that Burst SCS subsequently modulates descending serotonergic and noradrenergic pathways. The latter may explain the delayed wash-in and wash-out effect observed in experimental studies. Although not substantiated by clinical data, first anecdotal reports on a delayed wash-in of Burst SCS do exist. Additionally, results from a recent RCT found that Burst SCS microdosing, a paradigm that relies on the introduction of stimulation-off phases in-between stimulation-on phases, is as effective as standard Burst SCS, indeed indicating a delayed wash-out following Burst SCS [112]. The activation or deactivation of such a large supraspinal loop might take more time as compared to the fast antidromic spinal mechanism known to be pivotal in Tonic SCS (see section 2.3.1 and Figure 1). Activation of a supraspinal loop implicates signal transfer at various levels in the brain including thalamus [113], cortical 
brain areas, but also nuclei involved in the descending part of the loop like the periaqueductal grey, ventro-medio medial medulla and nucleus raphe $[36,49]$, as well as signal transfer and distribution over the various cortical areas or pain matrix [114].

That the mechanism underlying Burst SCS differs from Tonic SCS is further indicated by experimental studies on the effect of pulse amplitude and the suppression of mechanical hypersensitivity in a neuropathic rat model [111]. Burst SCS and mechanical hypersensitivity are characterized by a nonlinear relation effect, where Burst SCS is superior at an amplitude of $50 \%$ of motor threshold as compared to amplitudes of $33 \%$ and $66 \%$ of motor threshold. At the same time, the relation between pulse amplitude and effect with Tonic SCS is linear. Hence, the optimal Burst SCS amplitude (at 50\% of motor threshold) was comparable to Tonic SCS at the high intensity $(66 \%$ of motor threshold) for attenuating mechanical hypersensitivity, and interestingly, the charge delivered per second was much greater for Burst SCS than for Tonic SCS at comparable behavioral outcomes. From this it is suggested that with Burst SCS a complex, non-linear interplay between charge delivery, activation of neuronal elements and pain relief does exist $[108,111]$.

\subsection{Conclusions, future directions and research agenda}

SCS and in particular Tonic SCS has been shown to represent a safe and effective last-resort therapy for patients with pharmacologically refractory pain conditions, especially those with FBSS, CRPS and PDPN. Nevertheless, serious limitations exist (see section 2.3.5). Among the main limitations is that with Tonic SCS only $50-70 \%$ of patients with refractory neuropathic pain achieve pain reductions of $\geq 50 \%$, and the average pain reduction is restricted to approximately $50-60 \%$. Then there is also a loss of efficacy that occurs over short and long durations $[8,115,116]$. In order to overcome these limitations, research in the field of SCS and neuropathic pain recently introduced new stimulation locations like DRGS and new subsensory SCS paradigms like HF SCS and Burst SCS. This increases options for the neuropathic pain patient and at the same time allows the possibility for individual and personalized treatment strategies. As the mechanisms of action are only rudimentary understood, and as the efficacy in terms of pain relief with use of these new locations and 
new SCS paradigms is not significantly surpassing that achieved with Tonic SCS, further research is needed. This then should be based on an orchestrated interplay between (reproducible) experimental animal studies and well-designed large, (preferably) nonindustry sponsored clinical trials. In this context the following research questions and research directions, in line with those formulated by the IASP special interest group Neuromodulation, need to be addressed (=research agenda):

1. What are the segmental and supraspinal circuits involved in SCS? The use of modern, genetically identified cell types (optogenetics) allows further understanding of these circuits. The involvement and role of glial cells is needed and warrants further research [117].

2. How do different stimulation paradigms (i.e. variations in frequency and/or intensity and/or pulse width) affect the spinal and supraspinal circuits, and what is the impact of the total charge and charge per pulse? As not only HF SCS (see section 2.5.2) and Burst SCS (see section 2.5.3), but also other stimulation paradigms such as high-density (HD) SCS [118-120] and 3D-guided SCS [121] have shown great promise, both experimental studies and large randomized studies are needed to understand and confirm these first and preliminary findings. Also the use of closed-loop SCS devices capable of measuring evoked compound action potentials (ECAPs) is encouraged in order to better understand the relationship between stimulation, electrophysiological response, and neuromodulation, which may then have direct consequences for SCS design and programming [122].

3. Animal pain research should include operant behavioral testing and should no longer be exclusively based on paw withdrawal testing. Operant testing includes affectiveemotional and cognitive aspects of pain and will likely improve clinical translation of findings.

4. Implementation of imaging techniques ( $\mathrm{fMRI}, \mathrm{PET}-\mathrm{scan}$ ) and correlation of involvement of supraspinal circuits as related to various SCS paradigms and stimulation locations (DRGS) and their effect on pain relief are needed. 
5. It is of utmost importance to understand the anatomy of the dorsal column and the role of unmyelinated (nociceptive) fibers (see section 2.3.4)

As Tonic SCS has been shown to affect cortical processing and thalamo-cortical communication, and the fact that new SCS paradigms like Burst SCS may specifically activate the $\mathrm{m}$-STT and with that cortical brain areas involved in the motivational, affective and emotional components of pain, makes this therapy also interesting for treatment of pain related co-morbidities like depression and stress. These co-morbidities, also often difficult to treat pharmacologically, are known to be associated to activation of closely related or even similar cortical brain areas. Novel SCS paradigms, for instance Burst SCS, may form a serious future option for modulating and treating not only chronic neuropathic pain but also its comorbidities. 


\subsection{References}

1. Bouhassira D, Lanteri-Minet M, Attal N, Laurent B, Touboul C. Prevalence of chronic pain with neuropathic characteristics in the general population. Pain. 2008;136(3):380-7.

2. Scholz J, Finnerup NB, Attal N, Aziz Q, Baron R, Bennett MI, et al. The IASP classification of chronic pain for ICD-11: chronic neuropathic pain. Pain. 2019;160(1):53-9.

3. Attal $N$, Cruccu $G$, Baron $R$, Haanpaa $M$, Hansson $P$, Jensen TS, et al. EFNS guidelines on the pharmacological treatment of neuropathic pain: 2010 revision. Eur J Neurol. 2010;17(9):1113-e88.

4. Finnerup NB, Attal N, Haroutounian S, McNicol E, Baron R, Dworkin RH, et al. Pharmacotherapy for neuropathic pain in adults: a systematic review and meta-analysis. Lancet Neurol. 2015;14(2):162-73.

5. de Vos CC, Meier K, Zaalberg PB, Nijhuis HJ, Duyvendak W, Vesper J, et al. Spinal cord stimulation in patients with painful diabetic neuropathy: a multicentre randomized clinical trial. Pain. 2014;155(11):2426-31.

6. Slangen R, Schaper NC, Faber CG, Joosten EA, Dirksen CD, van Dongen RT, et al. Spinal cord stimulation and pain relief in painful diabetic peripheral neuropathy: a prospective two-center randomized controlled trial. Diabetes Care. 2014;37(11):3016-24.

7. Kemler MA, Barendse GA, van Kleef M, de Vet HC, Rijks CP, Furnee CA, et al. Spinal cord stimulation in patients with chronic reflex sympathetic dystrophy. N Engl J Med. 2000;343(9):618-24.

8. Kemler MA, de Vet HC, Barendse GA, van den Wildenberg FA, van Kleef M. Effect of spinal cord stimulation for chronic complex regional pain syndrome Type I: five-year final follow-up of patients in a randomized controlled trial. J Neurosurg. 2008;108(2):292-8.

9. Kumar K, Taylor RS, Jacques L, Eldabe S, Meglio M, Molet J, et al. Spinal cord stimulation versus conventional medical management for neuropathic pain: a multicentre randomised controlled trial in patients with failed back surgery syndrome. Pain. 2007;132(1-2):179-88.

10. North RB, Kumar K, Wallace MS, Henderson JM, Shipley J, Hernandez J, et al. Spinal cord stimulation versus re-operation in patients with failed back surgery syndrome: an international multicenter randomized controlled trial (EVIDENCE study). Neuromodulation. 2011;14(4):330-5; discussion 5-6.

11. Smits $\mathrm{H}$, van Kleef $\mathrm{M}$, Holsheimer J, Joosten EA. Experimental spinal cord stimulation and neuropathic pain: mechanism of action, technical aspects, and effectiveness. Pain Pract. 2013;13(2):154-68.

12. Meyerson BA, Linderoth B. Mode of action of spinal cord stimulation in neuropathic pain. J Pain Symptom Manage. 2006;31(4 Suppl):S6-12.

13. Shechter R, Yang F, Xu Q, Cheong YK, He SQ, Sdrulla A, et al. Conventional and kilohertz-frequency spinal cord stimulation produces intensity- and frequency-dependent inhibition of mechanical hypersensitivity in a rat model of neuropathic pain. Anesthesiology. 2013;119(2):422-32.

14. Miller JP, Eldabe S, Buchser E, Johanek LM, Guan Y, Linderoth B. Parameters of Spinal Cord Stimulation and Their Role in Electrical Charge Delivery: A Review. Neuromodulation. 2016;19(4):373-84.

15. Melzack R, Wall PD. Pain mechanisms: a new theory. Science. 1965;150(3699):971-9.

16. Shealy CN, Mortimer JT, Reswick JB. Electrical inhibition of pain by stimulation of the dorsal columns: preliminary clinical report. Anesth Analg. 1967;46(4):489-91.

17. Barolat G, Massaro F, He J, Zeme S, Ketcik B. Mapping of sensory responses to epidural stimulation of the intraspinal neural structures in man. J Neurosurg. 1993;78(2):233-9.

18. North RB, Ewend MG, Lawton MT, Piantadosi S. Spinal cord stimulation for chronic, intractable pain: superiority of "multi-channel" devices. Pain. 1991;44(2):119-30.

19. Janssen SP, Gerard S, Raijmakers ME, Truin M, Van Kleef M, Joosten EA. Decreased intracellular GABA levels contribute to spinal cord stimulation-induced analgesia in rats suffering from painful peripheral neuropathy: the role of KCC2 and GABA(A) receptor-mediated inhibition. Neurochem Int. 2012;60(1):21-30.

20. Linderoth B, Stiller CO, Gunasekera L, O'Connor WT, Ungerstedt U, Brodin E. Gamma-aminobutyric acid is released in the dorsal horn by electrical spinal cord stimulation: an in vivo microdialysis study in the rat. Neurosurgery. 1994;34(3):484-8; discussion 8-9.

21. Stiller CO, Cui JG, O'Connor WT, Brodin E, Meyerson BA, Linderoth B. Release of gamma-aminobutyric acid in the dorsal horn and suppression of tactile allodynia by spinal cord stimulation in mononeuropathic rats. Neurosurgery. 1996;39(2):367-74; discussion 74-5.

22. Cui JG, O'Connor WT, Ungerstedt U, Linderoth B, Meyerson BA. Spinal cord stimulation attenuates augmented dorsal horn release of excitatory amino acids in mononeuropathy via a GABAergic mechanism. Pain. 1997;73(1):87-95. 
23. Cui JG, Linderoth B, Meyerson BA. Effects of spinal cord stimulation on touch-evoked allodynia involve GABAergic mechanisms. An experimental study in the mononeuropathic rat. Pain. 1996;66(2-3):287-95.

24. Schechtmann G, Lind G, Winter J, Meyerson BA, Linderoth B. Intrathecal clonidine and baclofen enhance the pain-relieving effect of spinal cord stimulation: a comparative placebo-controlled, randomized trial. Neurosurgery. 2010;67(1):173-81.

25. Lind G, Meyerson BA, Winter J, Linderoth B. Intrathecal baclofen as adjuvant therapy to enhance the effect of spinal cord stimulation in neuropathic pain: a pilot study. Eur J Pain. 2004;8(4):377-83.

26. Lind G, Schechtmann G, Winter J, Meyerson BA, Linderoth B. Baclofen-enhanced spinal cord stimulation and intrathecal baclofen alone for neuropathic pain: Long-term outcome of a pilot study. Eur J Pain. 2008;12(1):1326.

27. Coull JA, Boudreau D, Bachand K, Prescott SA, Nault F, Sik A, et al. Trans-synaptic shift in anion gradient in spinal lamina I neurons as a mechanism of neuropathic pain. Nature. 2003;424(6951):938-42.

28. Janssen SP, Truin M, Van Kleef $M$, Joosten EA. Differential GABAergic disinhibition during the development of painful peripheral neuropathy. Neuroscience. 2011;184:183-94.

29. Woolf CJ, Thompson SW. The induction and maintenance of central sensitization is dependent on $\mathrm{N}$ methyl-D-aspartic acid receptor activation; implications for the treatment of post-injury pain hypersensitivity states. Pain. 1991;44(3):293-9.

30. Truin M, Janssen SP, van Kleef M, Joosten EA. Successful pain relief in non-responders to spinal cord stimulation: the combined use of ketamine and spinal cord stimulation. Eur J Pain. 2011;15(10):1049 e1-9.

31. Vranken JH, Troost D, Wegener JT, Kruis MR, van der Vegt MH. Neuropathological findings after continuous intrathecal administration of $\mathrm{S}(+)$-ketamine for the management of neuropathic cancer pain. Pain. 2005;117(1-2):231-5.

32. Guan Y, Wacnik PW, Yang F, Carteret AF, Chung CY, Meyer RA, et al. Spinal cord stimulation-induced analgesia: electrical stimulation of dorsal column and dorsal roots attenuates dorsal horn neuronal excitability in neuropathic rats. Anesthesiology. 2010;113(6):1392-405.

33. Sdrulla AD, Xu Q, He SQ, Tiwari V, Yang F, Zhang C, et al. Electrical stimulation of low-threshold afferent fibers induces a prolonged synaptic depression in lamina II dorsal horn neurons to high-threshold afferent inputs in mice. Pain. 2015;156(6):1008-17.

34. Sun L, Tai L, Qiu Q, Mitchell R, Fleetwood-Walker S, Joosten EA, et al. Endocannabinoid activation of CB1 receptors contributes to long-lasting reversal of neuropathic pain by repetitive spinal cord stimulation. Eur J Pain. 2017;21(5):804-14.

35. Inoue K, Tsuda M. Microglia in neuropathic pain: cellular and molecular mechanisms and therapeutic potential. Nat Rev Neurosci. 2018;19(3):138-52.

36. Millan MJ. Descending control of pain. Prog Neurobiol. 2002;66(6):355-474.

37. Saade NE, Tabet MS, Soueidan SA, Bitar M, Atweh SF, Jabbur SJ. Supraspinal modulation of nociception in awake rats by stimulation of the dorsal column nuclei. Brain Res. 1986;369(1-2):307-10.

38. Song Z, Ansah OB, Meyerson BA, Pertovaara A, Linderoth B. The rostroventromedial medulla is engaged in the effects of spinal cord stimulation in a rodent model of neuropathic pain. Neuroscience. 2013;247:134-44.

39. Song Z, Ultenius C, Meyerson BA, Linderoth B. Pain relief by spinal cord stimulation involves serotonergic mechanisms: an experimental study in a rat model of mononeuropathy. Pain. 2009;147(1-3):241-8.

40. Tazawa T, Kamiya Y, Kobayashi A, Saeki K, Takiguchi M, Nakahashi Y, et al. Spinal cord stimulation modulates supraspinal centers of the descending antinociceptive system in rats with unilateral spinal nerve injury. Mol Pain. 2015;11:36.

41. Wang R, King T, De Felice M, Guo W, Ossipov MH, Porreca F. Descending facilitation maintains long-term spontaneous neuropathic pain. J Pain. 2013;14(8):845-53.

42. Song Z, Meyerson BA, Linderoth B. Spinal 5-HT receptors that contribute to the pain-relieving effects of spinal cord stimulation in a rat model of neuropathy. Pain. 2011;152(7):1666-73.

43. El-Khoury C, Hawwa N, Baliki M, Atweh SF, Jabbur SJ, Saade NE. Attenuation of neuropathic pain by segmental and supraspinal activation of the dorsal column system in awake rats. Neuroscience. 2002;112(3):54153.

44. Meuwissen K, Van der Toorn A, Gu J, Zhang T, Dijkhuizen R, Joosten E. Burst and Tonic Spinal Cord Stimulation Engage Different Supraspinal Mechanisms: a Functional Magnetic Resonance Imaging Study in Peripherally Injured Chronic Neuropathic Rats. Under Review. 
45. Meuwissen KPV, van Beek M, Joosten EAJ. Burst and Tonic Spinal Cord Stimulation in the Mechanical Conflict-Avoidance System: Cognitive-Motivational Aspects. Neuromodulation. 2019.

46. Bentley LD, Duarte RV, Furlong PL, Ashford RL, Raphael JH. Brain activity modifications following spinal cord stimulation for chronic neuropathic pain: A systematic review. Eur J Pain. 2016;20(4):499-511.

47. Kiriakopoulos ET, Tasker RR, Nicosia S, Wood ML, Mikulis DJ. Functional magnetic resonance imaging: a potential tool for the evaluation of spinal cord stimulation: technical case report. Neurosurgery. 1997;41(2):501-4.

48. Kulkarni B, Bentley DE, Elliott R, Youell P, Watson A, Derbyshire SW, et al. Attention to pain localization and unpleasantness discriminates the functions of the medial and lateral pain systems. Eur J Neurosci. 2005;21(11):3133-42.

49. Basbaum Al, Bautista DM, Scherrer G, Julius D. Cellular and molecular mechanisms of pain. Cell. 2009;139(2):267-84.

50. Moens M, Sunaert S, Marien P, Brouns R, De Smedt A, Droogmans S, et al. Spinal cord stimulation modulates cerebral function: an fMRI study. Neuroradiology. 2012;54(12):1399-407.

51. Yezierski RP, Hansson P. Inflammatory and Neuropathic Pain From Bench to Bedside: What Went Wrong? J Pain. 2018;19(6):571-88.

52. Borsook D, Hargreaves R, Bountra C, Porreca F. Lost but making progress--Where will new analgesic drugs come from? Sci Transl Med. 2014;6(249):249sr3.

53. Vierck CJ, Hansson PT, Yezierski RP. Clinical and pre-clinical pain assessment: are we measuring the same thing? Pain. 2008;135(1-2):7-10.

54. Harte SE, Meyers JB, Donahue RR, Taylor BK, Morrow TJ. Mechanical Conflict System: A Novel Operant Method for the Assessment of Nociceptive Behavior. PLoS One. 2016;11(2):e0150164.

55. Smits $H$, van Kleef $M$, Joosten EA. Spinal cord stimulation of dorsal columns in a rat model of neuropathic pain: evidence for a segmental spinal mechanism of pain relief. Pain. 2012;153(1):177-83.

56. Smith KJ, Bennett BJ. Topographic and quantitative description of rat dorsal column fibres arising from the lumbar dorsal roots. J Anat. 1987;153:203-15.

57. Holsheimer J. Computer modelling of spinal cord stimulation and its contribution to therapeutic efficacy. Spinal Cord. 1998;36(8):531-40.

58. Holsheimer J, Khan YN, Raza SS, Khan EA. Effects of electrode positioning on perception threshold and paresthesia coverage in spinal cord stimulation. Neuromodulation. 2007;10(1):34-41.

59. Langford LA, Coggeshall RE. Unmyelinated axons in the posterior funiculi. Science. 1981;211(4478):176-

7.

60. Saliani A, Perraud B, Duval T, Stikov N, Rossignol S, Cohen-Adad J. Axon and Myelin Morphology in Animal and Human Spinal Cord. Front Neuroanat. 2017;11:129.

61. Niu J, Ding L, Li JJ, Kim H, Liu J, Li H, et al. Modality-based organization of ascending somatosensory axons in the direct dorsal column pathway. J Neurosci. 2013;33(45):17691-709.

62. Patterson JT, Head PA, McNeill DL, Chung K, Coggeshall RE. Ascending unmyelinated primary afferent fibers in the dorsal funiculus. J Comp Neurol. 1989;290(3):384-90.

63. Patterson JT, Chung K, Coggeshall RE. Further evidence for the existence of long ascending unmyelinated primary afferent fibers within the dorsal funiculus: effects of capsaicin. Pain. 1992;49(1):117-20.

64. Smith TM, Lee D, Bradley K, McMahon SB. Methodology for quantifying excitability of identified projection neurons in the dorsal horn of the spinal cord, specifically to study spinal cord stimulation paradigms. J Neurosci Methods. 2019;330:108479.

65. Liem L, Russo M, Huygen FJ, Van Buyten JP, Smet I, Verrills P, et al. A multicenter, prospective trial to assess the safety and performance of the spinal modulation dorsal root ganglion neurostimulator system in the treatment of chronic pain. Neuromodulation. 2013;16(5):471-82; discussion 82.

66. Huygen F, Liem L, Cusack W, Kramer J. Stimulation of the L2-L3 Dorsal Root Ganglia Induces Effective Pain Relief in the Low Back. Pain Pract. 2018;18(2):205-13.

67. Deer TR, Levy RM, Kramer J, Poree L, Amirdelfan K, Grigsby E, et al. Dorsal root ganglion stimulation yielded higher treatment success rate for complex regional pain syndrome and causalgia at 3 and 12 months: a randomized comparative trial. Pain. 2017;158(4):669-81.

68. Eldabe S, Burger K, Moser H, Klase D, Schu S, Wahlstedt A, et al. Dorsal Root Ganglion (DRG) Stimulation in the Treatment of Phantom Limb Pain (PLP). Neuromodulation. 2015;18(7):610-6; discussion 6-7. 
69. Eldabe S, Espinet A, Wahlstedt A, Kang P, Liem L, Patel NK, et al. Retrospective Case Series on the Treatment of Painful Diabetic Peripheral Neuropathy With Dorsal Root Ganglion Stimulation. Neuromodulation. 2018;21(8):787-92.

70. Levy RM, Mekhail N, Kramer J, Poree L, Amirdelfan K, Grigsby E, et al. Therapy Habituation at 12 Months: Spinal Cord Stimulation Versus Dorsal Root Ganglion Stimulation for Complex Regional Pain Syndrome Type I and II. J Pain. 2019.

71. Deer TR, Levy RM, Kramer J, Poree L, Amirdelfan K, Grigsby E, et al. Comparison of Paresthesia Coverage of Patient's Pain: Dorsal Root Ganglion vs. Spinal Cord Stimulation. An ACCURATE Study Sub-Analysis. Neuromodulation. 2019;22(8):930-6.

72. Mekhail N, Deer TR, Kramer J, Poree L, Amirdelfan K, Grigsby E, et al. Paresthesia-Free Dorsal Root Ganglion Stimulation: An ACCURATE Study Sub-Analysis. Neuromodulation. 2019.

73. Graham RD, Bruns TM, Duan B, Lempka SF. Dorsal root ganglion stimulation for chronic pain modulates Abeta-fiber activity but not C-fiber activity: A computational modeling study. Clin Neurophysiol. 2019;130(6):94151.

74. Koetsier E, Franken G, Debets J, Heijmans L, van Kuijk SMJ, Linderoth B, et al. Mechanism of dorsal root ganglion stimulation for pain relief in painful diabetic polyneuropathy is not dependent on GABA release in the dorsal horn of the spinal cord. CNS Neurosci Ther. 2020;26(1):136-43.

75. Koopmeiners AS, Mueller S, Kramer J, Hogan QH. Effect of electrical field stimulation on dorsal root ganglion neuronal function. Neuromodulation. 2013;16(4):304-11; discussion 10-1.

76. Kent AR, Min X, Hogan QH, Kramer JM. Mechanisms of Dorsal Root Ganglion Stimulation in Pain Suppression: A Computational Modeling Analysis. Neuromodulation. 2018;21(3):234-46.

77. Krames ES. The dorsal root ganglion in chronic pain and as a target for neuromodulation: a review. Neuromodulation. 2015;18(1):24-32; discussion

78. Du X, Hao H, Yang Y, Huang S, Wang C, Gigout S, et al. Local GABAergic signaling within sensory ganglia controls peripheral nociceptive transmission. J Clin Invest. 2017;127(5):1741-56.

79. Pawela CP, Kramer JM, Hogan QH. Dorsal root ganglion stimulation attenuates the BOLD signal response to noxious sensory input in specific brain regions: Insights into a possible mechanism for analgesia. Neuroimage. 2017;147:10-8.

80. Annemans L, Van Buyten JP, Smith T, Al-Kaisy A. Cost effectiveness of a novel $10 \mathrm{kHz}$ high-frequency spinal cord stimulation system in patients with failed back surgery syndrome (FBSS). J Long Term Eff Med Implants. 2014;24(2-3):173-83.

81. Van Buyten JP, Al-Kaisy A, Smet I, Palmisani S, Smith T. High-frequency spinal cord stimulation for the treatment of chronic back pain patients: results of a prospective multicenter European clinical study. Neuromodulation. 2013;16(1):59-65; discussion -6.

82. Song Z, Meyerson BA, Linderoth B. High-Frequency $(1 \mathrm{kHz})$ Spinal Cord Stimulation-Is Pulse Shape Crucial for the Efficacy? A Pilot Study. Neuromodulation. 2015;18(8):714-20.

83. Al-Kaisy A, Van Buyten JP, Smet I, Palmisani S, Pang D, Smith T. Sustained effectiveness of $10 \mathrm{kHz}$ highfrequency spinal cord stimulation for patients with chronic, low back pain: 24-month results of a prospective multicenter study. Pain Med. 2014;15(3):347-54.

84. Kriek N, Groeneweg G, Huygen FJ. Burst Spinal Cord Stimulation in a Patient with Complex Regional Pain Syndrome: A 2-year Follow-Up. Pain Pract. 2015;15(6):E59-64.

85. De Ridder D, Vanneste S. Burst and Tonic Spinal Cord Stimulation: Different and Common Brain Mechanisms. Neuromodulation. 2016;19(1):47-59.

86. De Ridder D, Vanneste S, Plazier M, van der Loo E, Menovsky T. Burst spinal cord stimulation: toward paresthesia-free pain suppression. Neurosurgery. 2010;66(5):986-90.

87. Schu S, Slotty PJ, Bara G, von Knop M, Edgar D, Vesper J. A prospective, randomised, double-blind, placebo-controlled study to examine the effectiveness of burst spinal cord stimulation patterns for the treatment of failed back surgery syndrome. Neuromodulation. 2014;17(5):443-50.

88. Kriek N, Groeneweg JG, Stronks DL, de Ridder D, Huygen FJ. Preferred frequencies and waveforms for spinal cord stimulation in patients with complex regional pain syndrome: A multicentre, double-blind, randomized and placebo-controlled crossover trial. Eur J Pain. 2017;21(3):507-19.

89. De Ridder D, Plazier M, Kamerling N, Menovsky T, Vanneste S. Burst spinal cord stimulation for limb and back pain. World Neurosurg. 2013;80(5):642-9 e1. 
90. Al-Kaisy A, Palmisani S, Pang D, Sanderson K, Wesley S, Tan Y, et al. Prospective, Randomized, ShamControl, Double Blind, Crossover Trial of Subthreshold Spinal Cord Stimulation at Various Kilohertz Frequencies in Subjects Suffering From Failed Back Surgery Syndrome (SCS Frequency Study). Neuromodulation. 2018;21(5):45765.

91. Perruchoud C, Eldabe S, Batterham AM, Madzinga G, Brookes M, Durrer A, et al. Analgesic efficacy of high-frequency spinal cord stimulation: a randomized double-blind placebo-controlled study. Neuromodulation. 2013;16(4):363-9; discussion 9.

92. Kapural L, Yu C, Doust MW, Gliner BE, Vallejo R, Sitzman BT, et al. Novel 10-kHz High-frequency Therapy (HF10 Therapy) Is Superior to Traditional Low-frequency Spinal Cord Stimulation for the Treatment of Chronic Back and Leg Pain: The SENZA-RCT Randomized Controlled Trial. Anesthesiology. 2015;123(4):851-60.

93. Lempka SF, McIntyre CC, Kilgore KL, Machado AG. Computational analysis of kilohertz frequency spinal cord stimulation for chronic pain management. Anesthesiology. 2015;122(6):1362-76.

94. Kilgore KL, Bhadra N. Reversible nerve conduction block using kilohertz frequency alternating current. Neuromodulation. 2014;17(3):242-54; discussion 54-5.

95. Chakravarthy K, Richter H, Christo PJ, Williams K, Guan Y. Spinal Cord Stimulation for Treating Chronic Pain: Reviewing Preclinical and Clinical Data on Paresthesia-Free High-Frequency Therapy. Neuromodulation. 2018;21(1):10-8.

96. Song Z, Viisanen H, Meyerson BA, Pertovaara A, Linderoth B. Efficacy of kilohertz-frequency and conventional spinal cord stimulation in rat models of different pain conditions. Neuromodulation. 2014;17(3):22634; discussion 34-5.

97. Arle JE, Mei L, Carlson KW, Shils JL. High-Frequency Stimulation of Dorsal Column Axons: Potential Underlying Mechanism of Paresthesia-Free Neuropathic Pain Relief. Neuromodulation. 2016;19(4):385-97.

98. Yang F, Carteret AF, Wacnik PW, Chung CY, Xing L, Dong X, et al. Bipolar spinal cord stimulation attenuates mechanical hypersensitivity at an intensity that activates a small portion of A-fiber afferents in spinal nerve-injured rats. Neuroscience. 2011;199:470-80.

99. Al-Kaisy A, Palmisani S, Smith T, Harris S, Pang D. The use of 10-kilohertz spinal cord stimulation in a cohort of patients with chronic neuropathic limb pain refractory to medical management. Neuromodulation. 2015;18(1):18-23; discussion

100. Amir R, Michaelis M, Devor M. Burst discharge in primary sensory neurons: triggered by subthreshold oscillations, maintained by depolarizing afterpotentials. J Neurosci. 2002;22(3):1187-98.

101. Wu G, Ringkamp M, Hartke TV, Murinson BB, Campbell JN, Griffin JW, et al. Early onset of spontaneous activity in uninjured C-fiber nociceptors after injury to neighboring nerve fibers. J Neurosci. 2001;21(8):RC140.

102. Wu G, Ringkamp M, Murinson BB, Pogatzki EM, Hartke TV, Weerahandi HM, et al. Degeneration of myelinated efferent fibers induces spontaneous activity in uninjured C-fiber afferents. J Neurosci. 2002;22(17):774653.

103. Emmers R. Thalamic mechanisms that process a temporal pulse code for pain. Brain Res. 1976;103(3):425-41.

104. Radhakrishnan V, Tsoukatos J, Davis KD, Tasker RR, Lozano AM, Dostrovsky JO. A comparison of the burst activity of lateral thalamic neurons in chronic pain and non-pain patients. Pain. 1999;80(3):567-75.

105. Lenz FA, Garonzik IM, Zirh TA, Dougherty PM. Neuronal activity in the region of the thalamic principal sensory nucleus (ventralis caudalis) in patients with pain following amputations. Neuroscience. 1998;86(4):1065-81. 106. Gu JW, Joosten EAJ. Clarifying the Scientific Knowledge Pertaining to Burst Waveforms in Spinal Cord Stimulation. Neuromodulation. 2019;22(6):758-9.

107. Tang R, Martinez M, Goodman-Keiser M, Farber JP, Qin C, Foreman RD. Comparison of burst and tonic spinal cord stimulation on spinal neural processing in an animal model. Neuromodulation. 2014;17(2):143-51.

108. Crosby ND, Goodman Keiser MD, Smith JR, Zeeman ME, Winkelstein BA. Stimulation parameters define the effectiveness of burst spinal cord stimulation in a rat model of neuropathic pain. Neuromodulation. 2015;18(1):1-8; discussion

109. Meuwissen KPV, de Vries LE, Gu JW, Zhang TC, Joosten EAJ. Burst and Tonic Spinal Cord Stimulation Both Activate Spinal GABAergic Mechanisms to Attenuate Pain in a Rat Model of Chronic Neuropathic Pain. Pain Pract. 2019.

110. Meuwissen KPV, Gu JW, Zhang TC, Joosten EAJ. Burst Spinal Cord Stimulation in Peripherally Injured Chronic Neuropathic Rats: A Delayed Effect. Pain Pract. 2018;18(8):988-96. 
111. Meuwissen KPV, Gu JW, Zhang TC, Joosten EAJ. Conventional-SCS vs. Burst-SCS and the Behavioral Effect on Mechanical Hypersensitivity in a Rat Model of Chronic Neuropathic Pain: Effect of Amplitude. Neuromodulation. 2018;21(1):19-30.

112. Vesper J, Slotty P, Schu S, Poeggel-Kraemer K, Littges H, Van Looy P, et al. Burst SCS Microdosing Is as Efficacious as Standard Burst SCS in Treating Chronic Back and Leg Pain: Results From a Randomized Controlled Trial. Neuromodulation. 2019;22(2):190-3.

113. Melzack R, Casey KL. Localized temperature changes evoked in the brain by somatic stimulation. Exp Neurol. 1967;17(3):276-92.

114. Martucci KT, Mackey SC. Neuroimaging of Pain: Human Evidence and Clinical Relevance of Central Nervous System Processes and Modulation. Anesthesiology. 2018;128(6):1241-54.

115. van Beek M, Geurts JW, Slangen R, Schaper NC, Faber CG, Joosten EA, et al. Severity of Neuropathy Is Associated With Long-term Spinal Cord Stimulation Outcome in Painful Diabetic Peripheral Neuropathy: Five-Year Follow-up of a Prospective Two-Center Clinical Trial. Diabetes Care. 2018;41(1):32-8.

116. Aiudi CM, Dunn RY, Burns SM, Roth SA, Opalacz A, Zhang Y, et al. Loss of Efficacy to Spinal Cord Stimulator Therapy: Clinical Evidence and Possible Causes. Pain Physician. 2017;20(7):E1073-E80.

117. Vallejo R, Gupta A, Kelley CA, Vallejo A, Rink J, Williams JM, et al. Effects of Phase Polarity and Charge Balance Spinal Cord Stimulation on Behavior and Gene Expression in a Rat Model of Neuropathic Pain. Neuromodulation. 2020;23(1):26-35.

118. Provenzano DA, Rebman J, Kuhel C, Trenz H, Kilgore J. The Efficacy of High-Density Spinal Cord Stimulation Among Trial, Implant, and Conversion Patients: A Retrospective Case Series. Neuromodulation. 2017;20(7):654-60.

119. Sweet J, Badjatiya A, Tan D, Miller J. Paresthesia-Free High-Density Spinal Cord Stimulation for Postlaminectomy Syndrome in a Prescreened Population: A Prospective Case Series. Neuromodulation. 2016;19(3):260-7.

120. Wille F, Breel JS, Bakker EW, Hollmann MW. Altering Conventional to High Density Spinal Cord Stimulation: An Energy Dose-Response Relationship in Neuropathic Pain Therapy. Neuromodulation. 2017;20(1):7180.

121. Veizi E, Hayek SM, North J, Brent Chafin T, Yearwood TL, Raso L, et al. Spinal Cord Stimulation (SCS) with Anatomically Guided (3D) Neural Targeting Shows Superior Chronic Axial Low Back Pain Relief Compared to Traditional SCS-LUMINA Study. Pain Med. 2017;18(8):1534-48.

122. Parker JL, Obradovic M, Hesam Shariati N, Gorman RB, Karantonis DM, Single PS, et al. Evoked Compound Action Potentials Reveal Spinal Cord Dorsal Column Neuroanatomy. Neuromodulation. 2020;23(1):82-95. 


\section{CHAPTER}

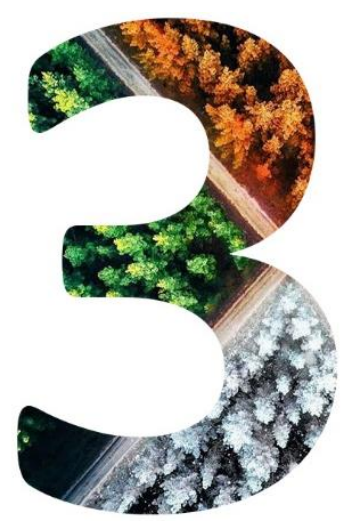

Dorsal Root Ganglion Stimulation in

Experimental Painful Diabetic Peripheral

Neuropathy: Burst versus Conventional

\section{Stimulation Paradigm}

G. Franken, J. Debets, E.A. Joosten

Published. Neuromodulation: Technology at the Neural Interface 2019; 22(8): 943-50. 


\subsection{Abstract}

Objectives: Painful diabetic peripheral neuropathy (PDPN) is a long-term complication of diabetes mellitus (DM). Dorsal Root Ganglion Stimulation (DRGS) has recently emerged as a neuromodulation modality in the treatment of chronic neuropathic pain. The objective of this study was to compare the effect of burst DRGS (Burst-DRGS) and conventional DRGS (Con-DRGS) in an experimental model of PDPN.

Methods: DM was induced in female Sprague-Dawley rats by intraperitoneal injection of streptozotocin (STZ, $n=48$ ). Animals were tested for mechanical hypersensitivity (50\% hind paw withdrawal threshold (WT) on Von Frey test) before, and 4 weeks after STZ injection. PDPN rats were then implanted with a unilateral bipolar lead at the L5 DRG $(n=22)$ and were stimulated for 30 minutes at days 2 and 3 post-implantation. Animals received ConDRGS and Burst-DRGS in a randomized crossover design $(n=10)$, or received Sham-DRGS $(n=7)$ for 30 minutes, and were tested for mechanical hypersensitivity at baseline, 15 and 30 minutes during DRGS, and 15 and 30 minutes following DRGS. Five animals were withdrawn from the study due to electrode-related technical problems.

Results: Con-DRGS and Burst-DRGS normalized STZ-induced mechanical hypersensitivity at 15 and 30 minutes during stimulation. A significant difference in terms of mechanical hypersensitivity was observed between both of the stimulated groups and the Sham-DRGS group at 15 and 30 minutes during stimulation. Interestingly, Burst-DRGS showed signs of a residual effect at 15 minutes after cessation of stimulation, while this was not the case for Con-DRGS.

Conclusions: Under the conditions tested, Con-DRGS and Burst-DRGS are equally effective in attenuating STZ-induced mechanical hypersensitivity in an animal model of PDPN. Burst-DRGS showed signs of a residual effect at 15 minutes after cessation of stimulation, which requires further investigation.

Keywords: Dorsal root ganglion stimulation, burst stimulation, painful diabetic peripheral neuropathy, mechanical hypersensitivity, neuropathic pain 


\subsection{Introduction}

Painful diabetic peripheral neuropathy (PDPN) is a debilitating consequence of diabetes mellitus (DM), with a prevalence ranging from 10-26\% [1-3]. PDPN typically presents as burning, electric, stabbing or tingling neuropathic pain that starts in the lower limbs, and is characterized by diffuse damage to small nerve fibers, specifically to those of the $A \delta$ and $C$ type [4]. Numerous pharmacological drugs for neuropathic pain have been introduced over the years [5]. As the efficacy of pharmacological drugs in PDPN is limited, there is an urgent need for the development of novel treatment options.

Spinal cord stimulation of the dorsal columns (SCS) is a recommended last resort therapy for PDPN patients who do not respond to conventional pharmacological medication. The effectiveness of SCS in PDPN has been demonstrated in two randomized clinical trials (RCTs) [6-8]. Despite considerable improvements, there are limitations to the efficacy of SCS. Firstly, approximately $60 \%$ of patients with PDPN achieve pain reductions of $\geq 50 \%$ [6-8]. Secondly, SCS is often unable to satisfactorily and specifically stimulate difficult-to-reach areas, such as the extremities in PDPN. Thirdly, placement of the leads on top of the dorsal columns makes SCS with conventional settings susceptible to postural variations due to changes in distance between stimulation lead and stimulation target, leading to unpleasant paresthesias and/or overstimulation [9]. Lastly, the energy consumption of SCS is relatively high, as there is significant energy loss to surroundings, such as the cerebrospinal fluid (CSF), before stimulation reaches the spinal cord dorsal columns.

In the field of neuromodulation, new developments aimed to change not only the anatomical target but also to introduce new stimulation waveforms have been suggested to overcome most of the disadvantages of SCS. Change of anatomical target from the spinal cord to the dorsal root ganglion (dorsal root ganglion stimulation, DRGS) not only increased treatment success in chronic intractable neuropathic pain but also resulted in less postural variation in paresthesia intensity and less battery consumption as compared to SCS [10]. Additionally, DRGS was found to be a safe and effective neuromodulation modality that improves painful symptoms in PDPN patients [11]. The introduction of new stimulation 
waveforms including use of burst paradigms [12] have been shown to result in clinically relevant pain reductions, without eliciting paresthesias. Studies have shown that burst SCS (Burst-SCS) decreases pain intensity to a greater degree than conventional SCS (Con-SCS) [13, 14]. This superior effect might be attributed to the underlying mechanism of action of BurstSCS, as it is hypothesized that Burst-SCS, besides targeting brain areas related to the location and intensity of pain (lateral pain pathway), also targets areas related to the emotional and affective components of pain (medial pain pathway) [13].

Over the years, preclinical research has provided valuable information with regard to the therapeutic effects of neuromodulation for PDPN. The effectiveness of SCS in experimental PDPN has been demonstrated in both the short $[15,16]$ and long term [17]. Interestingly, the first in vivo study to test the effectiveness of DRGS in an animal model of peripheral nerve injury was recently published [18]. The authors showed that DRGS attenuated both reflex-based pain behavior as well as affective pain behavior, with no signs of histological damage to the DRG.

In line with the recent changes in the field of neuromodulation, a combination of novel anatomical targeting and the use of novel stimulation waveforms might provide a platform to further improve neuromodulatory therapies for chronic neuropathic pain. To date, no study has explored the effect of novel DRGS modalities, like Burst-DRGS, in experimental or clinical PDPN. The present study therefore aimed to assess the effect of Burst-DRGS vs. Con-DRGS in an animal model of PDPN. To this end, we used an animal model for DRGS, which was demonstrated to relieve neuropathic pain in rats [18]. Based on the aforementioned evidence on Burst stimulation in the field of SCS, we hypothesized that Burst-DRGS leads to significantly higher pain relief and higher responder rates compared to Con-DRGS. 


\subsection{Methods}

\subsubsection{Ethical statement}

All experiments were conducted in a humane manner in accordance with the European Directive for the Protection of Vertebrate Animals Used for Experimental and Other Scientific Purposes (86/609/EU). The study was approved by the Animal Research Committee of Maastricht University (DEC-protocol 2013-079).

\subsubsection{Animals}

All experiments were performed using young-adult, female Sprague-Dawley rats (6 weeks at study onset, 180-220g, $n=48$ ). Animals were housed per 2 in filter-top polycarbonate cages in a climate controlled room (temperature $21 \pm 1^{\circ} \mathrm{C}$, humidity $55 \pm 15 \%$ ) with constant background music (approximately 45 decibel) and under artificial lightning (12:12 reversed light/dark cycle). Distilled water and food was at all times available to the animals ad libitum. Animals were allowed to acclimatize to the housing facility without experimenter contact for 1 week after arrival, and were handled properly before the onset of the experiments.

\subsubsection{Induction of Diabetes Mellitus (DM)}

Diabetes mellitus (DM) was induced by a single intraperitoneal injection of $65 \mathrm{mg} / \mathrm{kg}$ Streptozotocin (STZ) (Sigma-Aldrich, Schnelldorf, Germany) ( $n=48)$. Prior to STZ injection, animals were weighed and fasted overnight. STZ was then freshly dissolved in sterile $0.9 \%$ $\mathrm{NaCl}$ to a solution of $65 \mathrm{mg} / \mathrm{mL}$. In the first week after STZ injection, blood glucose levels were assessed in blood derived from the saphenous vein using a blood glucose meter (AccuChek Aviva ${ }^{\circledR}$, Roche Diagnostics GmbH, Mannheim, Germany). Rats with a glucose level of $\geq 15 \mathrm{mmol} / \mathrm{L}$ were considered diabetic [19] and were included in the study. When glucose levels exceeded $31.4 \mathrm{mmol} / \mathrm{L}$, one-third of a slow releasing insulin pellet (LinShin Canada, Inc.) was placed subcutaneously in the trunk of the animal. 


\subsubsection{Assessment of mechanical hypersensitivity (Von Frey assay)}

Mechanical hypersensitivity was assessed by measuring the response of the hind paws to Von Frey filaments using the 'up-down' method [20]. In short, rats were placed in a transparent box on an elevated mesh floor. Animals were allowed to acclimate to the behavioral set-up for 15 minutes before testing. Subsequently, a series of Von Frey filaments with incrementing stiffness (bending forces $0.6,1.2,2.0,3.6,5.5,8.5,15.1$, and $28.84 \mathrm{~g}$ ) were applied to the plantar surface of the hind paws of the animals for $5 \mathrm{~s}$. In case of a negative response (no withdrawal of hind paw), the next filament with higher bending force was applied. In case of a positive response (withdrawal of hind paw), the previous filament with lower bending force was applied. The $50 \%$ withdrawal threshold (WT) was calculated after completion of a sequence of six consecutive responses. A cut-off value of $28.84 \mathrm{~g}$ was defined to prevent tissue damage. Lastly, the $50 \%$ WT was multiplied by 10.000 and logarithmically transformed to account for Weber's law [21] and obtain a linear scale.

\subsubsection{Development of mechanical hypersensitivity}

Only animals showing mechanical hypersensitivity on the Von Frey assay at 4 weeks after STZ injection were implanted and treated with DRGS. Animals without mechanical hypersensitivity were excluded from the study. The presence of mechanical hypersensitivity was defined as a decrease of $\geq 0.2$ unit in $\log _{10}(10.000 \times 50 \%$ WT $)$ when compared to pre-STZ values [15-17].

\subsubsection{Dorsal root ganglion stimulation (DRGS) lead}

Preparation of the dorsal root ganglion stimulation (DRGS) lead was performed as previously described [18]. Briefly, the lead was manufactured out of two platinum-iridium wires with different diameters (0.010 and 0.005 in). The insulation at the termini of both wires was removed, and the terminal of the large wire $(0.010 \mathrm{in})$ was bent back upon itself to produce an atraumatic tip. The smaller wire $(0.005 \mathrm{in})$ was then wrapped around the insulated part of the larger wire. A few spots of dental cement were added to strengthen the lead. Lastly, the lead was tested with an Ohmmeter to confirm proper functioning of the lead (Figure 1). 


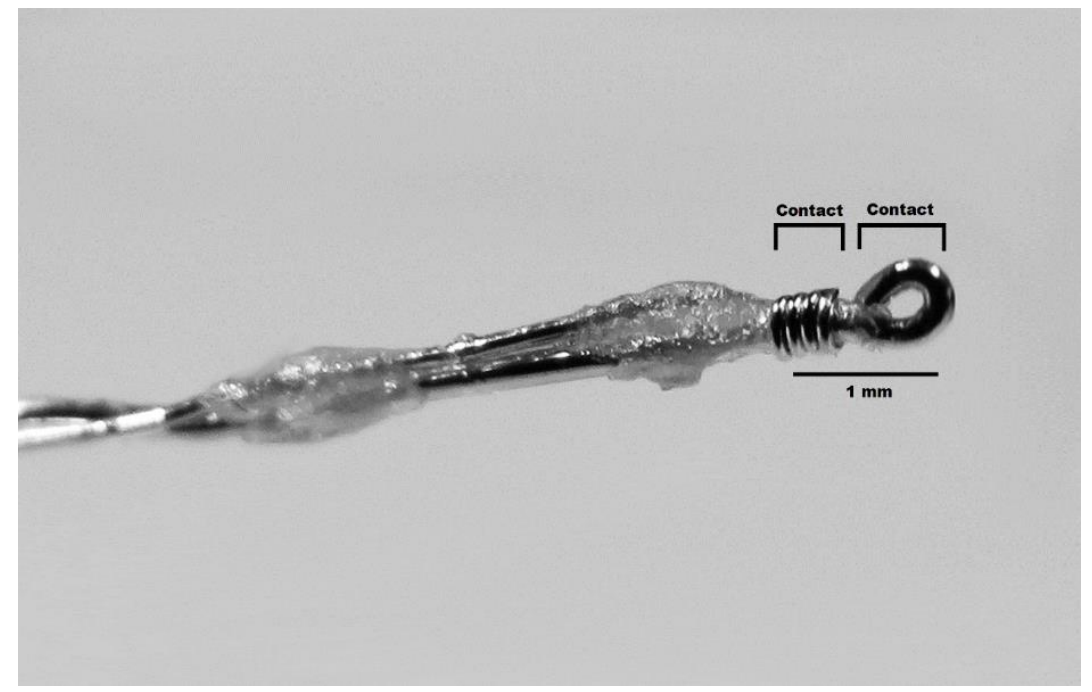

Figure 1. Bipolar DRGS lead.

\subsubsection{Implantation of the DRGS lead}

Implantation of the DRGS lead at the L5 DRG was performed as previously described [18]. Briefly, the intervertebral foramen at the level of the fifth lumbar (L5) spinal nerve was exposed via a paravertebral incision under general anesthesia. Subsequently, the foramen was gently opened by probing with a small, blunt nerve hook to provide a passage for the lead to enter the foramen on the dorsolateral aspect of the L5 DRG. The lead was secured onto the transverse process caudal to the foramen using a stainless steel ligature and a small screw (diameter $0.86 \mathrm{~mm}$, length $3.2 \mathrm{~mm}$ ). This produced a device capable of providing bipolar contact in apposition to the L5 DRG. Lastly, the lead was tunneled subcutaneously through the neck of the animals and the wounds were closed in layers. After implantation of the lead, the rats were allowed to recover for two days before the start of DRGS.

\subsubsection{DRG stimulation (DRGS)}

For stimulation of the L5 DRG, an A-M systems stimulator (MultiStim: Programmable 8-Channel Stimulator (Model 3800) $220 \mathrm{~V} / 50 \mathrm{~Hz}$ ) fitted with an additional stimulus Isolator (Model 3820 for A-M Systems MultiStim) was used. After connecting the implanted lead to 
the stimulator, the motor threshold (MT) was determined using a frequency of $2 \mathrm{~Hz}$ and pulse width of $200 \mu \mathrm{s}$ for Con-DRGS, and a pulse width of $1000 \mu \mathrm{s}$, five pulses (500 Hz intraburst frequency) administered at an interburst frequency of $2 \mathrm{~Hz}$ for Burst-DRGS. MT was defined as the current inducing contractions of the lower trunk or hind limb(s). For Con-DRGS the stimulation settings were as follows: biphasic stimulation with frequency $=50 \mathrm{~Hz}$, pulse width $=200 \mu \mathrm{s}$, amplitude $=67 \%$ of motor threshold (Figure 2a). For Burst-DRGS the stimulation settings were as follows: monophasic stimulation with interburst frequency= $40 \mathrm{~Hz}$, intraburst frequency $=500 \mathrm{~Hz}$, pulse width $=1000 \mu \mathrm{s}$, interpulse interval $=1000 \mu \mathrm{s}$, burst pulse count $=5$, amplitude $=67 \% \mathrm{MT}[12-14]$ (Figure 2b). Animals with an MT of $\geq 1 \mathrm{~mA}$ at stimulation days were excluded from analysis. For sham stimulated animals the amplitude was set at zero. Animals were unrestrained during DRGS.

A

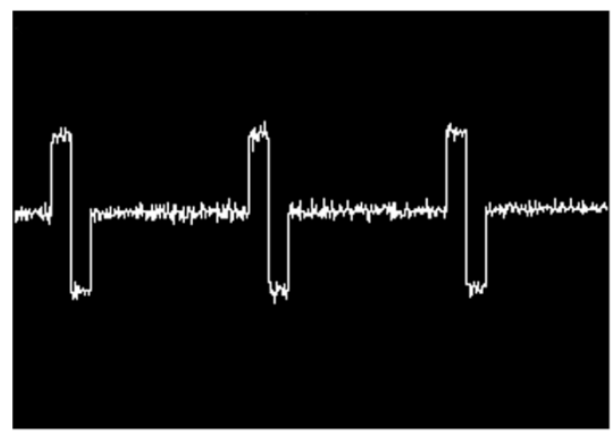

B

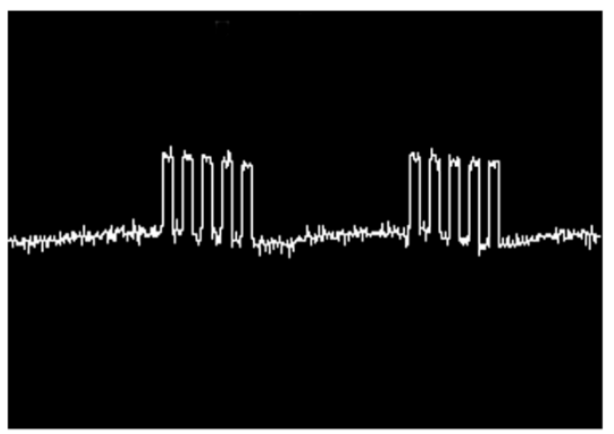

Figure 2. Oscilloscope output of the Con-DRGS (a) and Burst-DRGS (b) waveform. Con-DRGS: biphasic mode with frequency $=50 \mathrm{~Hz}$, pulse width $=200 \mu \mathrm{sec}$. Burst-DRGS: monophasic mode with interburst frequency $=40 \mathrm{~Hz}$, intraburst frequency $=500 \mathrm{~Hz}$, pulse width $=1000 \mu \mathrm{sec}$, interpulse interval $=1000 \mu \mathrm{sec}$, burstpulse count $=5$.

\subsubsection{Timeline of experiments}

Following baseline measurements for mechanical hypersensitivity (Von Frey) (week -1), animals were injected with STZ (week 0). In the first week after STZ injection (week 1), blood glucose of the animals was measured to confirm DM (DM defined as blood glucose level $\geq 15 \mathrm{mmol} / \mathrm{L}$ [19]). 4 weeks after STZ injection (week 4), animals were again tested for 
mechanical hypersensitivity, in order to select animals that developed PDPN ( $\geq 0.2$ decrease in $\log _{10}(10.000 \times 50 \%$ WT) on Von Frey when compared to the pre-STZ baseline [15-17]) for DRGS implantation (week 5). PDPN animals received either Con-DRGS and Burst-DRGS in a randomized cross-over design on days 2 and 3 post-implantation, or were assigned to a Sham-DRGS group ( $50 \%$ WT measured on day 2). The experimenter was blinded for the DRGS paradigm used. On stimulation days, animals were first tested for MT, after which the amplitude was set accordingly. Animals were then tested for mechanical hypersensitivity on Von Frey just before DRGS onset (baseline), 15 and 30 minutes during DRGS (or sham-DRGS), and 15 and 30 minutes after DRGS (45 and 60 minutes) (Figure 3 ).

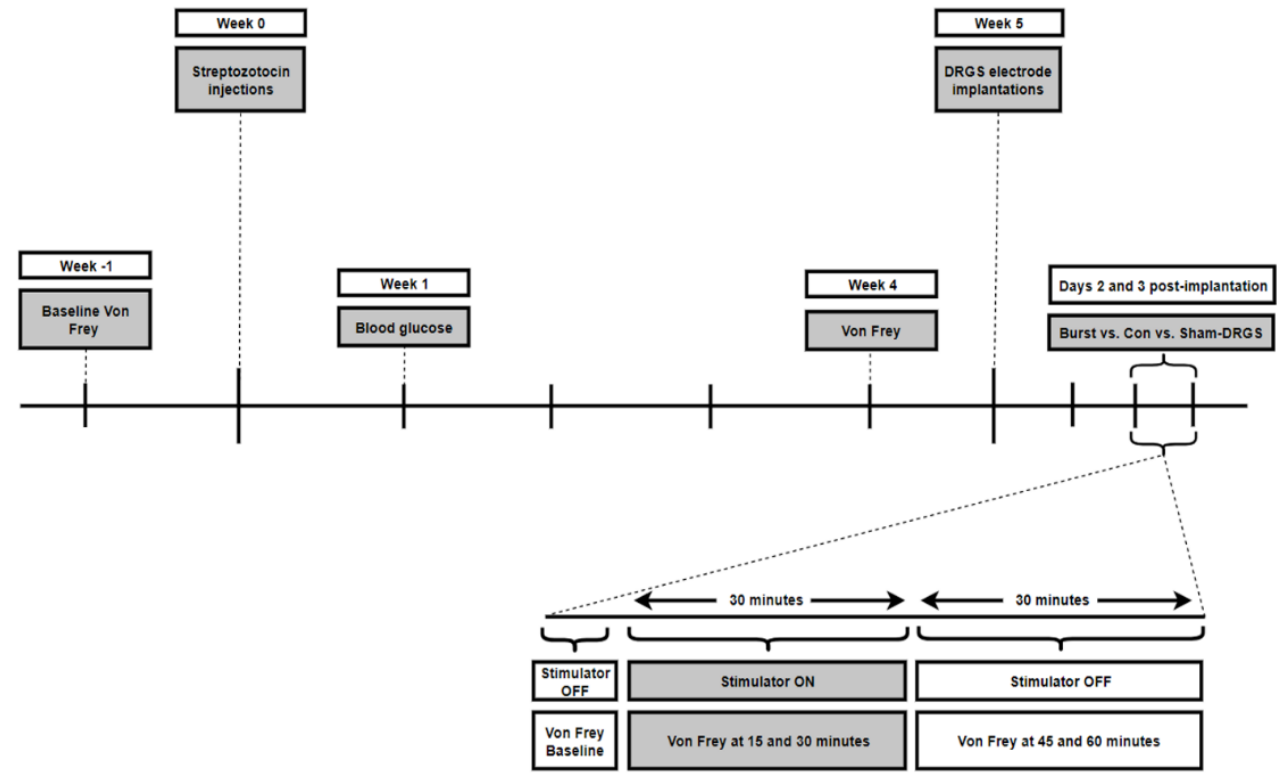

Figure 3. Timeline of experiments.

\subsubsection{Statistical analysis}

The WTs to Von Frey filaments are presented as mean \pm standard error of the mean (SEM). For statistical analysis, Von Frey data were logarithmically transformed to account for Weber's Law [21] and obtain a linear scale. Data were tested for a normal distribution using the Shapiro-Wilk normality test and were confirmed to be normally distributed. For analysis of intra-group changes in WTs over time, one-way repeated measures analysis of variance 
(ANOVA) was performed, followed by Tukey's multiple comparison test. For between-groups analysis (Con-DRGS vs. Burst-DRGS vs. Sham-DRGS), a two-way ANOVA followed by Tukey's multiple comparisons test was used. For comparisons between pre-STZ WTs and pre-implant WTs, and comparisons of MTs between the Con-DRGS and Burst-DRGS group, a pairedsamples t-test was used.

\subsection{Results}

\subsubsection{Flowchart of animals}

Out of the 48 animals that were injected with STZ, 43 developed Diabetes Mellitus (DM) (90\%; blood glucose level $\geq 15 \mathrm{mmol} / \mathrm{L})$. One animal died as a result from STZ-related health deterioration. 13 animals required insulin treatment (blood glucose level $\geq 31.4$ $\mathrm{mmol} / \mathrm{L}) .25$ out of the 43 diabetic animals developed subsequent PDPN 4 weeks post-STZ injection (58\%; $\geq 0.2$ decrease in $\log _{10}(10.000 \times 50 \%$ WT) on Von Frey when compared to the pre-STZ baseline [15-17]), of which 22 were successfully implanted with a DRGS device. Two out of the 22 implanted PDPN animals were withdrawn from the study due to connector breakage before the first stimulation day, one animal was withdrawn from the study due to not being able to finish the complete study period (no motor threshold observed on second stimulation day), and two animals were excluded from the study because of high MT (MT $\geq$ $1 \mathrm{~mA}$ ). Consequently, 17 animals were left for analysis. Of these 17 animals, 10 animals received Con-DRGS and Burst-DRGS in a randomized cross-over design, and 7 animals received Sham-DRGS.

\subsubsection{Development of STZ-induced mechanical hypersensitivity}

The mean $\log _{10}(10.000 \times 50 \%$ WT) value of the 17 stimulated animals (animals that underwent Con-DRGS, Burst-DRGS, or Sham-DRGS) dropped from $5.06 \pm 0.04$ before STZ injection to $4.47 \pm 0.04$ pre-implantation ( 4 weeks following STZ injection) $(p<0.0001$ ) (Figure 4). 


\section{Development of mechanical hypersensitivity}

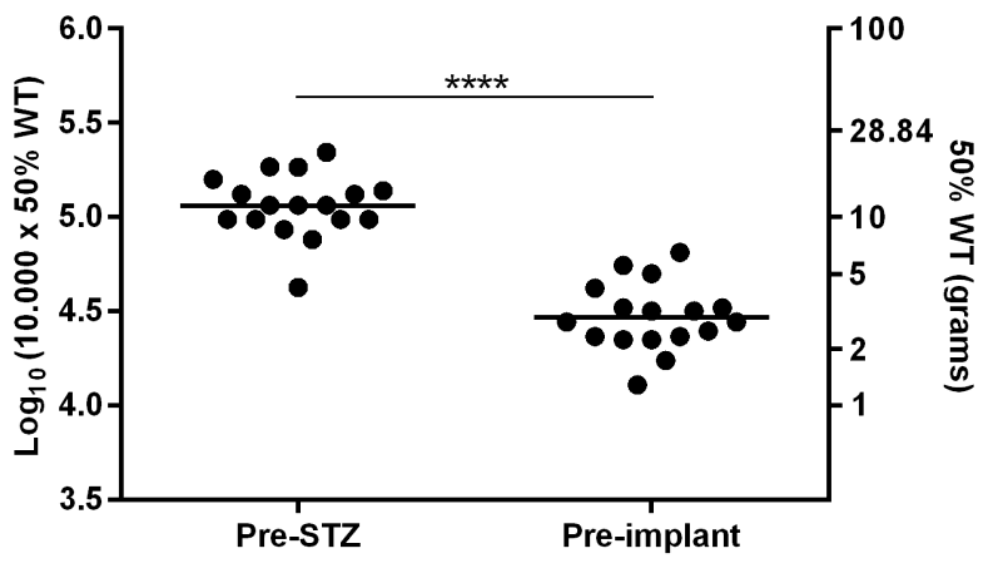

Figure 4. Development of mechanical hypersensitivity after STZ injection of all stimulated rats (animals that underwent Con-DRGS, Burst-DRGS, or Sham-DRGS; $\mathrm{n}=17$ ). ${ }^{* * * *} \mathrm{p}<0.0001$ compared to pre-STZ baseline.

\subsubsection{Effect of Con-DRGS on STZ-induced mechanical hypersensitivity}

For animals receiving Con-DRGS, the average baseline $\log _{10}(10.000 \times 50 \%$ WT) score (before start of Con-DRGS) was $4.38 \pm 0.07$. Con-DRGS resulted in a significant reduction of mechanical hypersensitivity at 15 minutes $(4.81 \pm 0.12, \mathrm{p}<0.05)$ and 30 minutes $(5.01 \pm 0.12$, $p<0.01)$, when compared to baseline. $\log _{10}(10.000 \times 50 \%$ WT) scores returned to baseline values after cessation of Con-DRGS at 45 minutes $(4.41 \pm 0.06, \mathrm{P}>0.99)$ and 60 minutes (4.42 $\pm 0.08, P>0.99$ ). No residual effect was observed for Con-DRGS, as both the 45 minutes and 60 minutes time point were significantly different compared to the 30 minutes time point $(p<0.01)$, while no significant difference was observed between the 45 and 60 minutes time points and baseline ( $p>0.99)$ (Figure 5a).

\subsubsection{Effect of Burst-DRGS on STZ-induced mechanical hypersensitivity}

For animals receiving Burst-DRGS, the average baseline $\log _{10}(10.000 \times 50 \% \mathrm{WT})$ score (before start of Burst-DRGS) was $4.31 \pm 0.06$. Burst-DRGS resulted in a significant reduction of mechanical hypersensitivity at 15 minutes $(4.91 \pm 0.13, p=0.01)$ and 30 minutes 
$(4.90 \pm 0.16, p=0.02)$, when compared to baseline. $\log _{10}(10.000 \times 50 \%$ WT) scores returned to baseline values after cessation of Burst-DRGS at 45 minutes $(4.58 \pm 0.15, p=0.40)$ and 60 minutes $(4.52 \pm 0.10, p=0.15)$. Importantly, Burst-DRGS showed signs of a residual effect at 45 minutes (15 minutes after stimulation), as there was no significant difference in the efficacy of Burst-DRGS at the 45 minutes time point when compared to the 30 minutes time point $(p>0.05)$ and baseline $(p=0.40)$ (Figure $5 b)$.

\subsubsection{Effect of Sham-DRGS on STZ-induced mechanical hypersensitivity}

For animals receiving Sham-DRGS, the average baseline $\log _{10}(10.000 \times 50 \%$ WT) score (before start of Sham-DRGS) was $4.42 \pm 0.04$. No significant differences in mechanical hypersensitivity were found at 15 minutes $(4.35 \pm 0.08, p=0.88), 30$ minutes $(4.38 \pm 0.13$, $\mathrm{p}>0.99), 45$ minutes $(4.35 \pm 0.10, \mathrm{p}=0.90)$, and 60 minutes $(4.38 \pm 0.12, p>0.99)$, when compared to baseline. Furthermore, no significant difference was observed between any of the tested time points ( $p>0.88$ ) (Figure $5 c$ ).

\subsubsection{Effect of Con-DRGS vs. Burst-DRGS vs. Sham-DRGS on STZ-induced mechanical hypersensitivity}

A significant difference between Con-DRGS and Sham-DRGS $\log _{10}(10.000 \times 50 \%$ WT) values was observed at 15 minutes of stimulation ( $4.81 \pm 0.12$ vs. $4.35 \pm 0.08, p=0.02)$ and 30 minutes of stimulation (5.01 \pm 0.12 vs. $4.38 \pm 0.13, p<0.001)$. No significant differences in terms of $\log _{10}(10.000 \times 50 \%$ WT) values were observed between Con-DRGS and Sham-DRGS at baseline $(4.38 \pm 0.07$ vs. $4.42 \pm 0.04, p=0.98), 45$ minutes $(4.41 \pm 0.06$ vs. $4.35 \pm 0.10$, $p=0.92)$, and 60 minutes ( $4.42 \pm 0.08$ vs. $4.38 \pm 0.12, p=0.97)$.

A significant difference was also observed between Burst-DRGS and Sham-DRGS $\log _{10}(10.000 \times 50 \% \mathrm{WT})$ values at 15 minutes of stimulation (4.91 \pm 0.13 vs. $4.35 \pm 0.08$, $p<0.01)$ and 30 minutes of stimulation ( $4.90 \pm 0.16$ vs. $4.38 \pm 0.13, p<0.01)$. No significant differences in terms of $\log _{10}(10.000 \times 50 \%$ WT $)$ values were observed between Burst-DRGS and Sham-DRGS at baseline ( $4.31 \pm 0.06$ vs. $4.42 \pm 0.04, p=0.81), 45$ minutes $(4.58 \pm 0.15$ vs. $4.35 \pm 0.10, p=0.32)$, and 60 minutes ( $4.52 \pm 0.10$ vs. $4.38 \pm 0.12, p=0.68)$. 
A

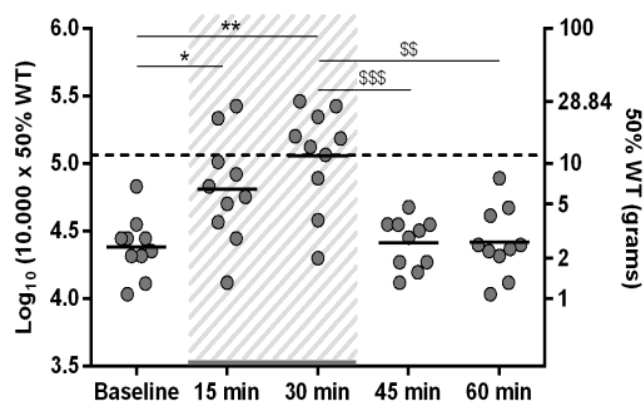

C

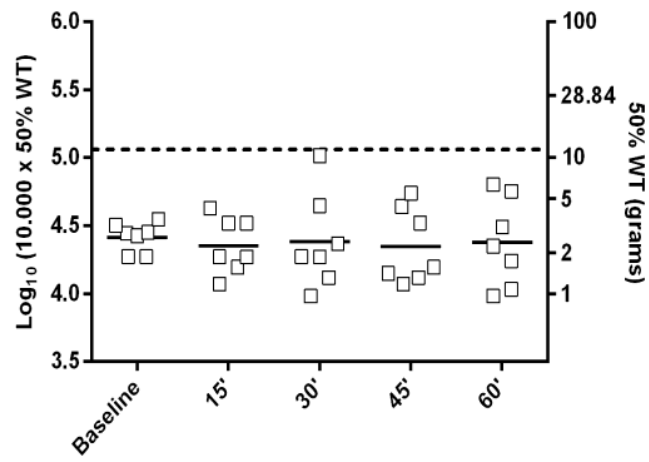

B Burst-DRGS

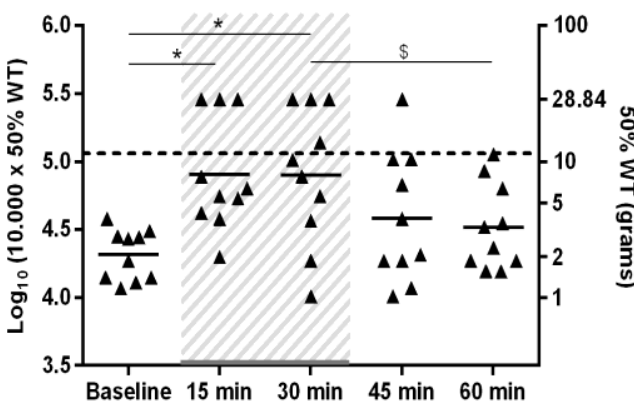

D Burst-DRGS vs. Con-DRGS vs. Sham-DRGS

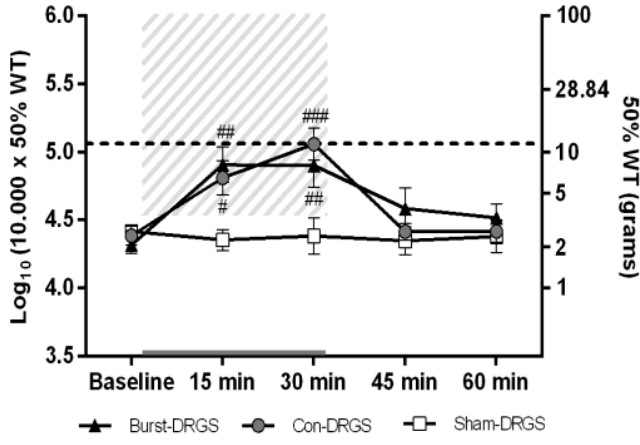

Figure 5. Scatter plot of the effect of Con-DRGS $(n=10)(a)$, Burst-DRGS $(n=10)(b)$, and Sham-DRGS $(n=7)(c)$ on STZ-induced mechanical hypersensitivity. A combined presentation of the effect of Con-DRGS, Burst-DRGS, and Sham-DRGS is presented in (d). Dotted line = the mean pre-STZ baseline of all stimulated animals. Gray area $=$ period of DRGS. ${ }^{*}, * * p<0.05, p<0.01$ compared to prestimulation baseline; $\$, \$ \$, \$ \$ p<0.05, p<0.01, p<$ 0.001 compared to $\mathrm{T}=30 \mathrm{~min}$; \#,\#, \#\#\#p<0.05, p<0.01, p $<0.001$ compared to the Sham-DRGS group at the same time point.

No significant differences were observed between Con-DRGS and Burst-DRGS at any time point: Baseline: $4.38 \pm 0.07$ vs. $4.31 \pm 0.06, p=0.88 ; 15$ minutes: $4.81 \pm 0.12$ vs. $4.91 \pm$ $0.13, p=0.80 ; 30$ minutes: $5.01 \pm 0.12$ vs. $4.90 \pm 0.16, p=0.55 ; 45$ minutes: $4.41 \pm 0.06$ vs. 4.58 $\pm 0.15, p=0.48$; 60 minutes: $4.42 \pm 0.08$ vs. $4.52 \pm 0.10, p=0.78$ (Figure $5 d$ ). Additionally, 
observed MTs were lower in the Burst-DRGS group compared to the Con-DRGS group, albeit not significant $(p=0.12)$ (Figure 6$)$.

\section{Motor thresholds}

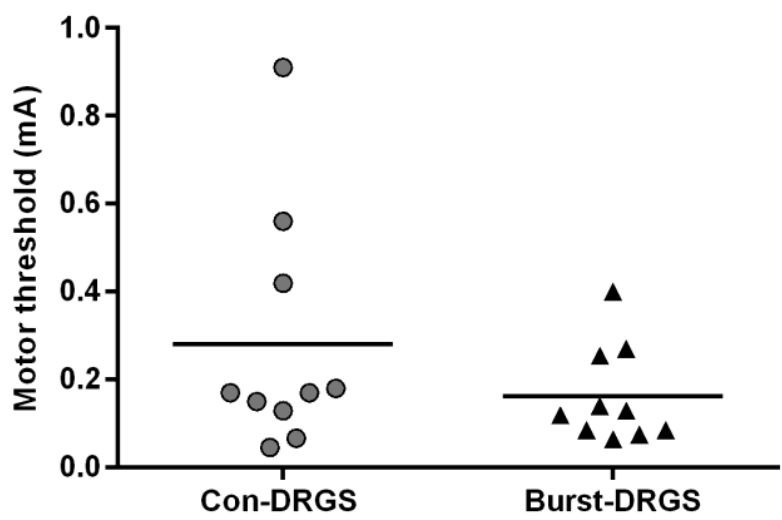

Figure 6. Motor thresholds (MT) assessed by means of Con-DRGS and Burst-DRGS. The MT of Con-DRGS was assessed at $2 \mathrm{~Hz}$ and pulse width of $200 \mu \mathrm{sec}$. The MT of Burst-DRGS was assessed using a pulse width of 1000 $\mu \mathrm{sec}$, and five pulses ( $500 \mathrm{~Hz}$ intraburst frequency) administered at an interburst frequency of $2 \mathrm{~Hz}$.

\subsubsection{Percentage responders: Con-DRGS vs. Burst-DRGS}

The percentage of responders to Con-DRGS was $70 \%$ (seven out of ten) at 15 minutes, $90 \%$ (nine out of ten) at 30 minutes of stimulation, $20 \%$ ( 2 out of ten) at 45 minutes, and $33 \%$ (3 out of ten) at 60 minutes. In the Burst-DRGS group, the percentage of responders was $80 \%$ (eight out of ten) at 15 minutes, $70 \%$ (seven out of ten) at 30 minutes of stimulation, $50 \%$ (five out of ten) at 45 minutes, and $40 \%$ ( 4 out of ten) at 60 minutes. A responder was defined as an animal with an increase of $\geq 0.2$ unit in $\log _{10}(10.000 \times 50 \%$ WT $)$ at the 15 minutes, 30 minutes, 45 minutes, or 60 minutes marks when compared to baseline before stimulation onset (Table 1).

Table 1. Percentage responders to DRGS: Con-DRGS vs. Burst-DRGS. A responder to stimulation is defined as an animal with an increase of the $10 \log (10.000 \times 50 \% \mathrm{WT}) \geq 0.2$ during stimulation.

$\begin{array}{lllll}\text { Group } & \mathbf{T}=\mathbf{1 5} \mathbf{m i n} & \mathbf{T}=\mathbf{3 0} \mathbf{~ m i n} & \mathbf{T}=\mathbf{4 5} \mathbf{~ m i n} & \mathbf{T}=\mathbf{6 0} \mathbf{~ m i n} \\ \text { Con-DRGS } & 7 / 10(70 \%) & 9 / 10(90 \%) & 2 / 10(20 \%) & 3 / 10(33 \%) \\ \text { Burst-DRGS } & 8 / 10(80 \%) & 7 / 10(70 \%) & 5 / 10(50 \%) & 4 / 10(40 \%)\end{array}$




\subsection{Discussion}

This is the first study to compare Burst-DRGS with Con-DRGS in PDPN. Our findings showed that both Con-DRGS and Burst-DRGS are equally effective in reversing STZ-induced mechanical hypersensitivity to pre-STZ baseline values. Since Con-DRGS was already very effective in bringing $\log _{10}(10.000 \times 50 \%$ WT $)$ values back to the healthy baseline level after 30 minutes of stimulation, it might have been challenging to show statistically significant improvements with Burst-DRGS over this effective Con-DRGS therapy. Nevertheless, BurstDRGS showed signs of a residual effect (not significant) at 15 minutes after cessation of stimulation, while this was not the case for Con-DRGS treated animals. Also the responder rates were considerably higher in the Burst-DRGS $(5 / 10 ; 50 \%)$ group when compared to the Con-DRGS group $(2 / 10 ; 20 \%) 15$ minutes after cessation of stimulation. Our results are in line with the work of Pan et al., who were the first to perform in vivo Con-DRGS in a unilateral peripheral nerve injury model of neuropathic pain [18]. The authors concluded that ConDRGS attenuates both reflex-based as well as affective pain behavior. Also the observed motor thresholds in the study of Pan et al (20) were comparable to those observed in our study. Importantly, the study of Pan and colleagues also showed that DRGS produces no signs of histological or behavioral injury to the DRG.

Also clinically, the effectiveness of Con-DRGS and the superiority of Con-DRGS over Con-SCS for the treatment of chronic intractable pain of the lower limbs attributed to complex regional pain syndrome (CRPS) and causalgia has been published in a randomized clinical trial (RCT)[10]. Additionally, the first retrospective study to assess the effect of ConDRGS for refractory PDPN patients was recently published by Eldabe et al. [11]. Despite the retrospective nature and small sample size (ten patients) of this study, the authors concluded that Con-DRGS is a safe and effective neuromodulation modality to improve painful symptoms in PDPN patients [11].

In the field of Burst stimulation, contradictory findings regarding the superiority of Burst-SCS over Con-SCS have been reported. While some studies show a clear advantage of Burst-SCS over Con-SCS [13, 14, 22], other studies show no difference between the two 
stimulation modalities in terms of their pain relieving effect [23], which may have resulted from the different disease indications assessed in these studies. The superiority of Burst-SCS observed in some studies might be attributed to the mechanism of action of Burst-SCS, as it is hypothesized that Burst-SCS, besides targeting brain areas related to the location and intensity of pain (lateral pain pathway), also targets areas related to the emotional and affective components of pain (medial pain pathway) [13]. Furthermore, a study by Tang et al. found that Burst-SCS at 60\% MT reduced neural activity significantly more than Con-SCS at the same amplitude, which might explain the superiority of Burst-SCS over Con-SCS [24]. Lastly, the total charge per second is higher with Burst-SCS when compared to Con-SCS, something that is hypothesized to correlate with stimulation efficacy [25]. In our study, no significant difference was found between Con-DRGS and Burst-DRGS on STZ-induced mechanical hypersensitivity. Nevertheless, the inclusion of only reflex-based tests in the present study might limit our window for detecting differences related to motivational affective aspects of pain [13]. Also, the Burst waveform used in the present study, albeit monophasic, varies slightly from the clinically-used BurstDR ${ }^{\mathrm{TM}}$ waveform, which is monophasic with a passive recharge balance.

The present preclinical DRGS model mimics features that are typical of clinical DRGS. As is the case in clinical DRGS, pain relief occurred very promptly (after 15 minutes of stimulation) in our animal model for both Con-DRGS and Burst-DRGS, and was maintained for the full 30 minutes stimulation period. Interestingly, a recent study by Meuwissen et al., which compared Con-SCS with Burst-SCS in an animal model of peripheral nerve injury, showed Burst-SCS to have a delayed onset and a delayed carry-over of analgesic effect when compared to Con-SCS [26]. While no differences in terms of a delayed onset of analgesic effect was observed between Con-DRGS and Burst-DRGS in the present study, Burst-DRGS appeared to show signs of a residual effect at 45 minutes when compared to Con-DRGS. Differences in the delayed wash-in of stimulation might be attributed to differences in the experimental model used, the location of stimulation (dorsal column vs. DRG), the type of stimulation (quadripolar vs. bipolar) and/or the Burst paradigm used (biphasic vs. monophasic). The motor thresholds necessary to evoke contractions of the hind paws of the 
animals were lower for Burst-DRGS than Con-DRGS in our study, albeit not significant. This is in line with preclinical findings that Burst-SCS requires significantly lower amplitudes to obtain a motor response when compared to Con-SCS [24, 26-30]. The latter has important consequences for the stimulation amplitude, which is generally lower with Burst-SCS when compared to Con-SCS [12].

To date, few studies have been conducted in order to elucidate the mechanism underlying DRGS. DRGS was found to inhibit neuronal excitability, by reducing the amplitude and/or the amount of action potentials arising from the DRG [31]. The unique pseudounipolar design and the T-junction of the DRG may act as a low-pass filter for electrical stimuli travelling from the periphery to the spinal cord [32]. Furthermore, it was shown by fMRI that DRGS is capable of attenuating BOLD signals in brain regions that are considered part of the pain matrix, like the contralateral thalamic VPL/VPM nuclei, and cortical S1 and S2 [33]. However, the involvement of the spinal pain gate should not be overlooked, as modulating firing rates of DRG neurons by DRGS may also affect interneurons and GABAergic systems in the dorsal horn as is the case in traditional SCS [34-36]. Interestingly, it was shown that BurstSCS does not rely on GABAergic mechanisms as is the case for Con-SCS, as it was shown that the effect of Con-SCS, but not Burst-SCS, is blocked by administration of a GABA-B receptor antagonist [28], suggesting that different mechanisms of action underlie different stimulation waveforms. Recently, a study by Du et al. found an extensive GABAergic communication network between sensory neuron somata inside the DRG [37]. The authors showed that sensory neurons in the DRG express major proteins required for GABA synthesis and release, and are capable of releasing GABA upon depolarization. Furthermore, it was found that local infusion of GABA reuptake inhibitors into the DRG alleviated neuropathic pain, whereas focal application of GABA receptor antagonists triggered neuropathic pain. The authors proposed the idea that this GABAergic system in the DRG acts as a second gate, in addition to the Gate Control Theory[38], and that neuromodulation of the DRG might exert its analgesic action by engaging this second gate [37]. More research into the underlying mechanisms of DRGS and its relation to specific stimulation waveforms is necessary to finetune DRGS for chronic neuropathic pain diseases. 
Limitations of this study include the use of only a short-term stimulation protocol. Long-term stimulation protocols, as being used in clinical practice, require further investigation. Secondly, only female Sprague-Dawley rats were included in our study as female Sprague-Dawley rats reach their maximal body weight and nerve conduction values faster and at a lower weight when compared to male Sprague-Dawley rats or either sex of other strains [19]. As there is strong evidence for sex differences in pain and analgesia, one should be cautious when extrapolating these data to the male sex [39]. As mentioned earlier, also the inclusion of only reflex-based tests has its limitations and the Burst waveform used in the present study varies slightly from the clinically used BurstDR ${ }^{\mathrm{TM}}$ waveform.

We conclude that under the conditions tested, Con-DRGS and Burst-DRGS are equally effective in attenuating streptozotocin-induced mechanical hypersensitivity in an animal model of PDPN. Importantly, Burst-DRGS showed signs of a residual effect at 15 minutes after cessation of stimulation, which was not the case with Con-DRGS. Further work needs to be done to confirm this residual effect of Burst-DRGS. The present study provides a first insight into the pain relieving effect of Burst-DRGS. Further optimization and analysis of DRGS driven by insights into the underlying mechanisms of the various stimulation paradigms is necessary. 


\subsection{References}

1. Abbott CA, Malik RA, van Ross ER, Kulkarni J, Boulton AJ. Prevalence and characteristics of painful diabetic neuropathy in a large community-based diabetic population in the U.K. Diabetes Care. 2011;34(10):2220-4.

2. Daousi C, MacFarlane IA, Woodward A, Nurmikko TJ, Bundred PE, Benbow SJ. Chronic painful peripheral neuropathy in an urban community: a controlled comparison of people with and without diabetes. Diabet Med. 2004;21(9):976-82.

3. Davies M, Brophy S, Williams R, Taylor A. The prevalence, severity, and impact of painful diabetic peripheral neuropathy in type 2 diabetes. Diabetes Care. 2006;29(7):1518-22.

4. Kaur S, Pandhi P, Dutta P. Painful diabetic neuropathy: an update. Ann Neurosci. 2011;18(4):168-75.

5. Finnerup NB, Otto M, Jensen TS, Sindrup SH. An evidence-based algorithm for the treatment of neuropathic pain. MedGenMed. 2007;9(2):36.

6. Slangen R, Schaper NC, Faber CG, Joosten EA, Dirksen CD, van Dongen RT, et al. Spinal cord stimulation and pain relief in painful diabetic peripheral neuropathy: a prospective two-center randomized controlled trial. Diabetes Care. 2014;37(11):3016-24.

7. van Beek M, Slangen R, Schaper NC, Faber CG, Joosten EA, Dirksen CD, et al. Sustained Treatment Effect of Spinal Cord Stimulation in Painful Diabetic Peripheral Neuropathy: 24-Month Follow-up of a Prospective TwoCenter Randomized Controlled Trial. Diabetes Care. 2015;38(9):e132-4.

8. de Vos CC, Meier K, Zaalberg PB, Nijhuis HJ, Duyvendak W, Vesper J, et al. Spinal cord stimulation in patients with painful diabetic neuropathy: a multicentre randomized clinical trial. Pain. 2014;155(11):2426-31.

9. Kramer J, Liem L, Russo M, Smet I, Van Buyten JP, Huygen F. Lack of body positional effects on paresthesias when stimulating the dorsal root ganglion (DRG) in the treatment of chronic pain. Neuromodulation. 2015;18(1):50-7; discussion 7.

10. Deer TR, Levy RM, Kramer J, Poree L, Amirdelfan K, Grigsby E, et al. Dorsal root ganglion stimulation yielded higher treatment success rate for complex regional pain syndrome and causalgia at 3 and 12 months: a randomized comparative trial. Pain. 2017;158(4):669-81.

11. Eldabe S, Espinet A, Wahlstedt A, Kang P, Liem L, Patel NK, et al. Retrospective Case Series on the Treatment of Painful Diabetic Peripheral Neuropathy With Dorsal Root Ganglion Stimulation. Neuromodulation. 2018.

12. De Ridder D, Vanneste S, Plazier M, van der Loo E, Menovsky T. Burst spinal cord stimulation: toward paresthesia-free pain suppression. Neurosurgery. 2010;66(5):986-90.

13. De Ridder D, Plazier M, Kamerling N, Menovsky T, Vanneste S. Burst spinal cord stimulation for limb and back pain. World Neurosurg. 2013;80(5):642-9 e1.

14. De Ridder D, Lenders MW, De Vos CC, Dijkstra-Scholten C, Wolters R, Vancamp T, et al. A 2-center comparative study on tonic versus burst spinal cord stimulation: amount of responders and amount of pain suppression. Clin J Pain. 2015;31(5):433-7.

15. Pluijms WA, van Kleef M, Honig WM, Janssen SP, Joosten EA. The effect of spinal cord stimulation frequency in experimental painful diabetic polyneuropathy. Eur J Pain. 2013;17(9):1338-46.

16. van Beek M, van Kleef M, Linderoth B, van Kuijk SM, Honig WM, Joosten EA. Spinal cord stimulation in experimental chronic painful diabetic polyneuropathy: Delayed effect of High-frequency stimulation. Eur J Pain. 2017;21(5):795-803.

17. van Beek M, Hermes D, Honig WM, Linderoth B, van Kuijk SMJ, van Kleef M, et al. Long-Term Spinal Cord Stimulation Alleviates Mechanical Hypersensitivity and Increases Peripheral Cutaneous Blood Perfusion in Experimental Painful Diabetic Polyneuropathy. Neuromodulation. 2018.

18. Pan B, Yu H, Fischer GJ, Kramer JM, Hogan QH. Dorsal Root Ganglionic Field Stimulation Relieves Spontaneous and Induced Neuropathic Pain in Rats. J Pain. 2016;17(12):1349-58.

19. Calcutt NA. Modeling diabetic sensory neuropathy in rats. Methods Mol Med. 2004;99:55-65.

20. Chaplan SR, Bach FW, Pogrel JW, Chung JM, Yaksh TL. Quantitative assessment of tactile allodynia in the rat paw. J Neurosci Methods. 1994;53(1):55-63.

21. Mills C, Leblond D, Joshi S, Zhu C, Hsieh G, Jacobson P, et al. Estimating efficacy and drug ED50's using von Frey thresholds: impact of weber's law and log transformation. J Pain. 2012;13(6):519-23.

22. de Vos CC, Bom MJ, Vanneste S, Lenders MW, de Ridder D. Burst spinal cord stimulation evaluated in patients with failed back surgery syndrome and painful diabetic neuropathy. Neuromodulation. 2014;17(2):152-9. 
23. Kriek N, Groeneweg JG, Stronks DL, de Ridder D, Huygen FJ. Preferred frequencies and waveforms for spinal cord stimulation in patients with complex regional pain syndrome: A multicentre, double-blind, randomized and placebo-controlled crossover trial. Eur J Pain. 2017;21(3):507-19.

24. Tang R, Martinez M, Goodman-Keiser M, Farber JP, Qin C, Foreman RD. Comparison of burst and tonic spinal cord stimulation on spinal neural processing in an animal model. Neuromodulation. 2014;17(2):143-51.

25. Miller JP, Eldabe S, Buchser E, Johanek LM, Guan Y, Linderoth B. Parameters of Spinal Cord Stimulation and Their Role in Electrical Charge Delivery: A Review. Neuromodulation. 2016;19(4):373-84.

26. Meuwissen KPV, Gu JW, Zhang TC, Joosten EAJ. Burst Spinal Cord Stimulation in Peripherally Injured Chronic Neuropathic Rats: A Delayed Effect. Pain Pract. 2018.

27. Gong WY, Johanek LM, Sluka KA. A Comparison of the Effects of Burst and Tonic Spinal Cord Stimulation on Hyperalgesia and Physical Activity in an Animal Model of Neuropathic Pain. Anesth Analg. 2016.

28. Crosby ND, Weisshaar CL, Smith JR, Zeeman ME, Goodman-Keiser MD, Winkelstein BA. Burst and Tonic Spinal Cord Stimulation Differentially Activate GABAergic Mechanisms to Attenuate Pain in a Rat Model of Cervical Radiculopathy. IEEE Trans Biomed Eng. 2015;62(6):1604-13.

29. Crosby ND, Goodman Keiser MD, Smith JR, Zeeman ME, Winkelstein BA. Stimulation parameters define the effectiveness of burst spinal cord stimulation in a rat model of neuropathic pain. Neuromodulation. 2015;18(1):1-8; discussion

30. Meuwissen KPV, Gu JW, Zhang TC, Joosten EAJ. Conventional-SCS vs. Burst-SCS and the Behavioral Effect on Mechanical Hypersensitivity in a Rat Model of Chronic Neuropathic Pain: Effect of Amplitude. Neuromodulation. 2018;21(1):19-30.

31. Koopmeiners AS, Mueller S, Kramer J, Hogan QH. Effect of electrical field stimulation on dorsal root ganglion neuronal function. Neuromodulation. 2013;16(4):304-11; discussion 10-1.

32. Krames ES. The dorsal root ganglion in chronic pain and as a target for neuromodulation: a review. Neuromodulation. 2015;18(1):24-32; discussion

33. Pawela CP, Kramer JM, Hogan QH. Dorsal root ganglion stimulation attenuates the BOLD signal response to noxious sensory input in specific brain regions: Insights into a possible mechanism for analgesia. Neuroimage. 2017;147:10-8.

34. Stiller CO, Cui JG, O'Connor WT, Brodin E, Meyerson BA, Linderoth B. Release of gamma-aminobutyric acid in the dorsal horn and suppression of tactile allodynia by spinal cord stimulation in mononeuropathic rats. Neurosurgery. 1996;39(2):367-74; discussion 74-5.

35. Cui JG, O'Connor WT, Ungerstedt U, Linderoth B, Meyerson BA. Spinal cord stimulation attenuates augmented dorsal horn release of excitatory amino acids in mononeuropathy via a GABAergic mechanism. Pain. 1997;73(1):87-95.

36. Janssen SP, Gerard S, Raijmakers ME, Truin M, Van Kleef M, Joosten EA. Decreased intracellular GABA levels contribute to spinal cord stimulation-induced analgesia in rats suffering from painful peripheral neuropathy: the role of KCC2 and GABA(A) receptor-mediated inhibition. Neurochem Int. 2012;60(1):21-30.

37. Du X, Hao H, Yang Y, Huang S, Wang C, Gigout S, et al. Local GABAergic signaling within sensory ganglia controls peripheral nociceptive transmission. J Clin Invest. 2017;127(5):1741-56.

38. Melzack R, Wall PD. Pain mechanisms: a new theory. Science. 1965;150(3699):971-9.

39. Craft RM, Mogil JS, Aloisi AM. Sex differences in pain and analgesia: the role of gonadal hormones. Eur J Pain. 2004;8(5):397-411. 


\title{
CHAPTER
}

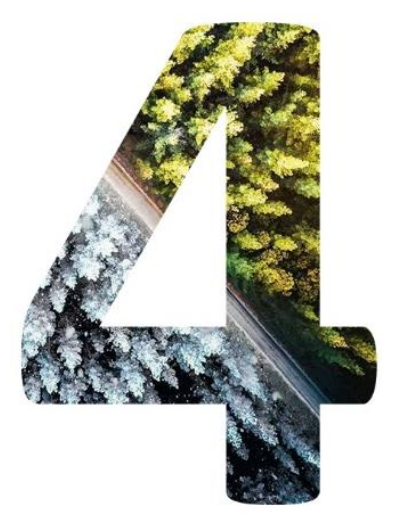

\section{Nonlinear Relation between Burst}

\section{Dorsal Root Ganglion Stimulation}

Amplitude and Behavioral Outcome in

an Experimental Model of Painful

Diabetic Peripheral Neuropathy

\author{
G. Franken, J. Debets, E.A. Joosten
}

Published. Neuromodulation: Technology at the Neural Interface 2020; 23(2): 158-66. 


\subsection{Abstract}

Background and objective: Dorsal root ganglion stimulation (DRGS) has recently emerged as a neuromodulation modality in the treatment of chronic neuropathic pain. The objective of this study was to compare the efficacy of different Burst-DRGS amplitudes in an experimental model of painful diabetic peripheral neuropathy (PDPN).

Methods: Diabetes Mellitus was induced in female Sprague-Dawley rats by intraperitoneal injection of streptozotocin (STZ, $n=28$ ). Animals were tested for mechanical hypersensitivity (von Frey paw withdrawal test) before, and 4 weeks after STZ injection. PDPN rats $(n=13)$ were implanted with a unilateral bipolar electrode at the L5 DRG. Animals received Burst-DRGS at $0 \%, 10 \%, 33 \%, 50 \%, 66 \%$ and $80 \%$ of motor threshold (MT) in a randomized crossover design on post-implantation days 2-7 $(n=9)$. Mechanical hypersensitivity was assessed before stimulation onset, 15 and 30 minutes during stimulation, and 15 and 30 minutes after stimulation.

Results: Burst-DRGS at amplitudes of $33 \%, 50 \%, 66 \%$, and $80 \% \mathrm{MT}$ resulted in significant attenuation of STZ-induced mechanical hypersensitivity at 15 and 30 minutes during stimulation, as well as 15 minutes after cessation of stimulation. No effect on mechanical hypersensitivity was observed for Burst-DRGS at 0\% MT and 10\% MT. Optimal pain relief and highest responder rates were achieved with Burst-DRGS at 50-66\% MT, with an estimated optimum at $52 \% \mathrm{MT}$.

Conclusion: Our findings indicate a nonlinear relationship between Burst-DRGS amplitude and behavioral outcome, with an estimated optimal amplitude of $52 \% \mathrm{MT}$. Further optimization and analysis of DRGS driven by insights into the underlying mechanisms related to the various stimulation paradigms is warranted.

Keywords: Dorsal root ganglion stimulation, burst stimulation, neuromodulation, painful diabetic peripheral neuropathy, neuropathic pain, Von Frey 


\subsection{Introduction}

Painful diabetic peripheral neuropathy (PDPN) is a frequent and disabling complication of Diabetes Mellitus (DM)[1-3]. In PDPN, small fibers of the $A_{\delta}$ and $C$ type are damaged, leading to subsequent neuropathic pain in extremities and often starting in the lower limbs[4]. As the effectiveness of pharmacological interventions in PDPN is limited[5], there is an urgent need for new non-pharmacological approaches. Spinal cord stimulation of the dorsal columns with conventional settings (Con-SCS) is an established last-resort treatment for PDPN patients who are refractory to pharmacological interventions. The effectiveness of Con-SCS in patients with PDPN has been demonstrated in two randomized clinical trials (RCTs)[6, 7]. However, despite considerable improvements with Con-SCS, ConSCS has limitations with regard to effect size, responder rate, specificity, stability, and energy consumption[6-11]. Recently, dorsal root ganglion stimulation (DRGS) was developed and expected to overcome some of the limitations observed with Con-SCS.

Since the implantation of the first fully implantable DRGS system in 2011[9], DRGS has shown clinical success in groin pain, axial back pain[12], leg and foot pain[9], complex regional pain syndrome (CRPS)[13], chest wall pain[14] and post-amputation pain syndromes[15]. Interestingly, a recent study by Eldabe and colleagues showed DRGS to be an effective and safe neuromodulation technique to improve painful symptoms in PDPN patients[16]. However, despite considerable improvements in terms of pain scores using DRGS, many PDPN patients still experience unsatisfactory pain relief ${ }^{16}$.

Besides changes in the anatomical target of stimulation, the introduction of novel stimulation waveforms has also aided to the field of SCS in recent years. SCS paradigms that use bursting patterns[17] offer a paresthesia-free solution to conventional Con-SCS, and decrease pain intensity to a greater degree than Con-SCS in some studies[18, 19]. Nevertheless, only few studies have investigated the combination of both novel anatomical targeting such as DRGS, and the use of novel stimulation waveforms, such as burst stimulation. 
In a previous experimental study, we showed that Burst-DRGS in a rat model of PDPN showed signs of a residual effect after cessation of stimulation, which was accompanied with higher responder rates when compared to conventional DRGS (ConDRGS)[20]. Although the results of this study already seem to favor Burst-DRGS over ConDRGS, there is still room for improvement in terms of the optimal stimulation settings of Burst-DRGS. Previous experimental electrophysiological and behavioral studies suggest that Burst-SCS can be optimized by changing stimulation settings that are related to the amount of energy delivered to the system, such as amplitude and pulse width[21-23]. This study aims to investigate the effect of stimulation intensity on the therapeutic efficacy or pain relief of Burst-DRGS in an experimental rat model of PDPN.

\subsection{Methods}

\subsubsection{Ethical statement}

All experiments were performed in accordance with the European Directive for the Protection of Vertebrate Animals Used for Experimental and Other Scientific Purposes (86/609/EU). The study was approved by the Central Authority for Scientific Procedures on Animals, The Netherlands (project license 2017-022).

\subsubsection{Animals}

Experiments were conducted in female Sprague-Dawley rats $(170-210 \mathrm{~g}$ at study onset; $n=28$ ). Animals were housed in pairs in polycarbonate cages placed in a controlled environment (temperature $21 \pm 1^{\circ} \mathrm{C}$, humidity $55 \pm 15 \%$ ) in a reversed day/night cycle. Animals had ad libitum access to distilled water and food. Before onset of the experiments, the animals were habituated to the housing facility for one week without experimenter contact.

\subsubsection{Induction of DM}

DM was induced by intraperitoneal injection of Streptozotocin (STZ; Sigma-Aldrich, Schnelldorf, Germany). Animals were weighed and fasted overnight, after which STZ was freshly dissolved in $0.9 \% \mathrm{NaCl}$ to achieve a solution of $65 \mathrm{mg} / \mathrm{mL}$. $65 \mathrm{mg} / \mathrm{kg} \mathrm{STZ}$ was then injected in order to induce DM. One week following STZ injection, animals were tested for 
blood glucose levels via blood derived from the saphenous vein (Accu-Chek Aviva ${ }^{\circledR}$, Roche Diagnostics $\mathrm{GmbH}$, Mannheim, Germany). Rats with a blood glucose level of $\geq 15 \mathrm{mmol} / \mathrm{L}$ were considered diabetic[24]. In case of excessively high glucose values (>31.4 mmol/L), onethird of a slow releasing insulin pellet (LinShin Canada, Inc.) was placed subcutaneously in the trunk of the animal.

\subsubsection{Assessment of mechanical hypersensitivity}

Mechanical hypersensitivity was assessed by determining the paw withdrawal threshold of the hind paw of the animals to calibrated Von Frey filaments. In short, animals were places on an elevated mesh floor in a transparent box. Animals were allowed to acclimatize to the testing environment for $15 \mathrm{~min}$. A series of calibrated Von Frey filaments $(0.6,1.2,2.0,3.6,5.5,8.5,15.1$, and $28.84 \mathrm{~g})$ were then applied to the plantar surface of the hind paw for 5 s using the "up-down" method[25]. The 50\% withdrawal threshold (WT) was calculated after completion of a sequence of six consecutive responses. A cut-off value of $28.84 \mathrm{~g}$ was defined to prevent tissue damage. Lastly, the $50 \%$ WT was multiplied by 10,000 and logarithmically transformed to account for Weber's law and obtain a linear scale[26].

Only animals displaying mechanical hypersensitivity at 4 weeks after STZ injection were implanted and treated with DRGS. Animals without mechanical hypersensitivity were excluded from the study. The presence of mechanical hypersensitivity was defined as a decrease of $\geq 0.2$ unit in $\log _{10}(10,000 \times 50 \%$ WT $)$ when compared to pre-STZ values [27-29].

\subsubsection{DRGS}

For DRGS, the lead fashioned out of two platinum-iridium wires (diameter 0.010 and 0.005 in) custom made for experimental studies, was unilaterally implanted adjacent to the left L5 DRG as previously described[20, 30]. Via paravertebral incision the intervertebral foramen was exposed at the level of the L5 spinal nerve. The entry of the lead into the foramen was ensured by a blunt nerve hook gently probing inside the foramen. Then the lead was inserted into the foramen and secured to the transverse process of L6 using a small stainless steel wire and screw (diameter $0.86 \mathrm{~mm}$, length $3.2 \mathrm{~mm}$ ). Lastly, the lead was subcutaneously tunneled through the neck of the animal, after which the wounds were 
closed in layers. Animals were allowed to recover for two days before starting the stimulation protocol.

Animals received Burst-DRGS using an Abbott Inc. Proclaim implantable pulse generator (IPG) at $0 \%, 10 \%, 33 \%, 50 \%, 66 \%$ and $80 \%$ of motor threshold (MT) at days $2-7$ following implantation (one amplitude each day) using a randomized crossover design. Randomization was performed by an independent researcher using the website randomize.org. The experimenter was blinded for the applied amplitude. On stimulation days, animals were externally connected to the IPG and tested for MT after which the amplitude was set accordingly for each animal. The MT was determined using an interburst frequency of $10 \mathrm{~Hz}$, intraburst frequency of $500 \mathrm{~Hz}$, pulse width of $1000 \mu \mathrm{s}$, interpulse interval of $1000 \mu$ s and a burst pulse count of 5. MT was defined as the current inducing contractions of the lower trunk or hind limb. Animals with an MT $\geq 1 \mathrm{~mA}$ were excluded from analysis. Settings for Burst-DRGS were as follows: monophasic stimulation with interburst frequency= $40 \mathrm{~Hz}$, intraburst frequency $=500 \mathrm{~Hz}$, pulse width $=1000 \mu \mathrm{s}$, interpulse interval $=1000 \mu \mathrm{s}$, burst pulse count= 5)[17-19] (Figure 1). Animals were not restrained during Burst-DRGS.

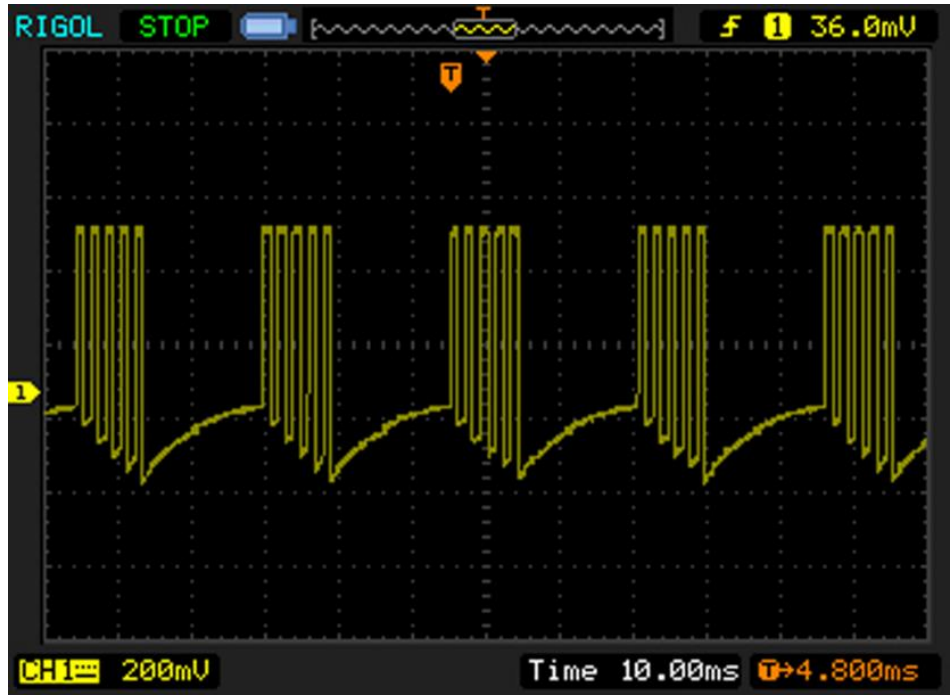

Figure 1. Oscilloscope recording of Burst-DRGS waveform. Settings for Burst-DRGS were as follows: monophasic stimulation with interburst frequency $=40 \mathrm{~Hz}$, intraburst frequency $=500 \mathrm{~Hz}$, pulse width $=1000 \mu \mathrm{sec}$, interpulse interval $=1000 \mu \mathrm{sec}$, burst pulse count $=5$. 


\subsubsection{Timeline of experiments}

Animals were tested for mechanical hypersensitivity (Von Frey) at baseline (week 1), after which animals were injected with STZ at week 0 . Blood was taken from the animals at week 1 to confirm induction of DM (defined as blood glucose level $\geq 15 \mathrm{mmol} / \mathrm{L}[24]$ ). Animals were again tested for mechanical hypersensitivity (Von Frey) at week 4, in order to select animals that developed PDPN (defined as $\geq 0.2$ decrease in $\log 10(10,000 \times 50 \%$ WT) on Von Frey test when compared to pre-STZ baseline [27-29]). Animals that developed PDPN were then implanted with a DRGS lead at week 5, and stimulated on days 2-7 following implantation using different amplitudes (0\%, 10\%, 33\%, 50\%, 66\% and 80\% MT). Animals were each day tested for mechanical hypersensitivity on Von Frey just before Burst-DRGS onset (baseline), 15 and 30 minutes during Burst-DRGS, and 15 and 30 minutes after BurstDRGS (45 and 60 minutes) (Figure 2).

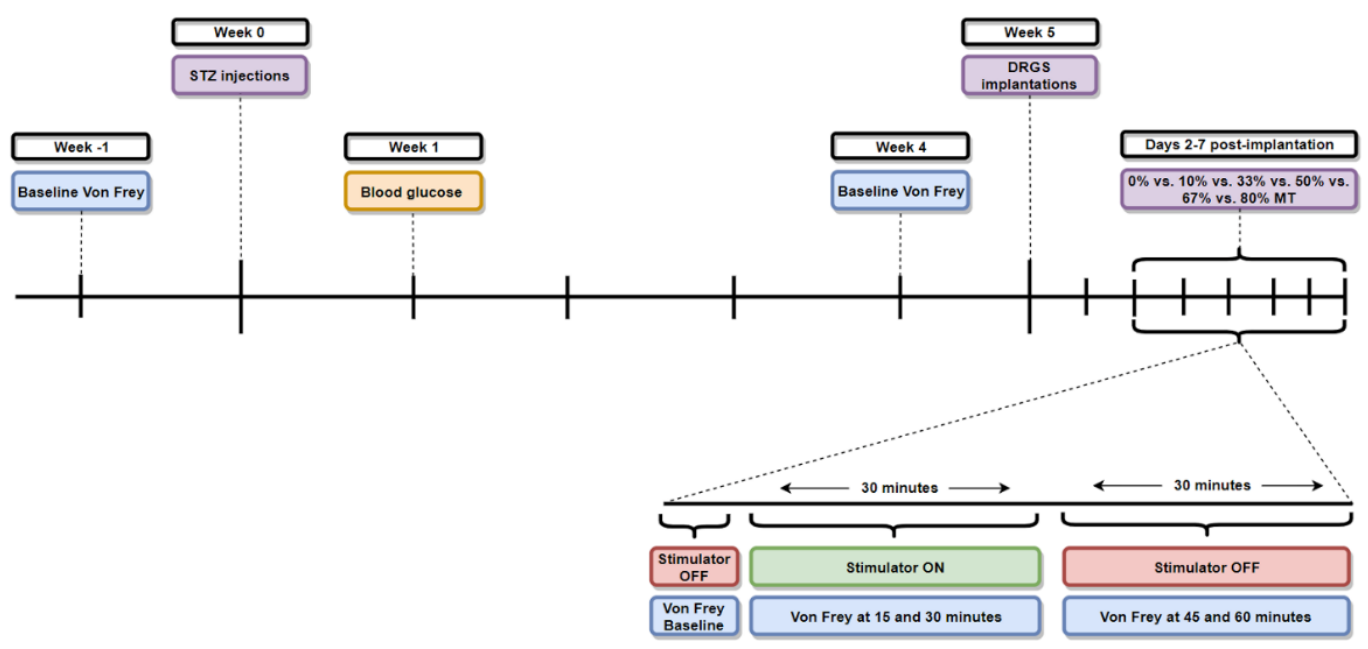

Figure 2. Study design.

\subsubsection{Data analysis}

Values are presented as mean \pm standard error of the mean (SEM). For statistical analysis, Von Frey data were logarithmically transformed to account for Weber's Law and obtain a linear scale[26]. For analysis of the effect of Burst-DRGS over time and intra-group changes in WTs over time, two-way repeated measures analysis of variance (ANOVA) was 
performed, followed by Dunnett's multiple comparison test. For between-groups analyses, a two-way ANOVA followed by Tukey's multiple comparisons test was used. For comparisons between pre-STZ WTs and pre-implant WTs, a paired-samples t-test was used. For the analysis of MTs over time, a one-way repeated measures ANOVA with Dunnett's multiple comparisons test was used. For calculation of the amplitude-mechanical hypersensitivity relationship at each specific time point (baseline, $15 \mathrm{~min}, 30 \mathrm{~min}, 45 \mathrm{~min}$ and $60 \mathrm{~min}$ ), mean $\log 10(10,000 \times 50 \%$ WT) values at each time point were expressed as a function of BurstDRGS amplitude. Non-linear regression was then performed for each time point, and the Xvalue corresponding to the vertex of the resulting parabola-shaped curve was defined as the optimal DRGS amplitude for that time point. For calculation of the overall optimal DRGS amplitude, area under the curve analysis was first performed for the effect of Burst-DRGS over time for each specific animal, at all tested Burst-DRGS amplitudes. The mean AUC values \pm SEM were then expressed as a function of Burst-DRGS amplitude, after which nonlinear regression was performed, and the X-value corresponding to the vertex of the parabola was identified as the overall optimal Burst-DRGS amplitude.

\subsection{Results}

\subsubsection{Flowchart of animals}

Out of the 28 STZ-injected animals, 27 animals developed Diabetes Mellitus (96\%; blood glucose level $\geq 15 \mathrm{mmol} / \mathrm{L}$ ). 7 animals required insulin treatment (blood glucose level $\geq$ $31.4 \mathrm{mmol} / \mathrm{L}$ ). 13 out of these 27 diabetic animals developed subsequent PDPN (48\%; $\geq 0.2$ decrease in $\log _{10}(10,000 \times 50 \%$ WT) on Von Frey when compared to the pre-STZ injection baseline). All PDPN animals ( $n=13$ ) were then implanted with a unilateral DRGS device at the

L5 lumbar level. Two animals were withdrawn from the study due to excessively high MT $(>1 \mathrm{~mA})$ and two animals were withdrawn due to not displaying neuropathic symptoms following implantation. In the end, 9 of these 13 implanted animals successfully finished the 6-day stimulation protocol. 


\subsubsection{Development of STZ-induced mechanical hypersensitivity}

The mean $\log _{10}(10,000 \times 50 \%$ WT) of the 13 implanted animals dropped from $5.1 \pm$ $0.05 \mathrm{~g}$ at pre-STZ-baseline to $4.7 \pm 0.05 \mathrm{~g}$ pre-implantation (4 weeks after STZ injection) $(P<0.0001)$ (Figure 3).

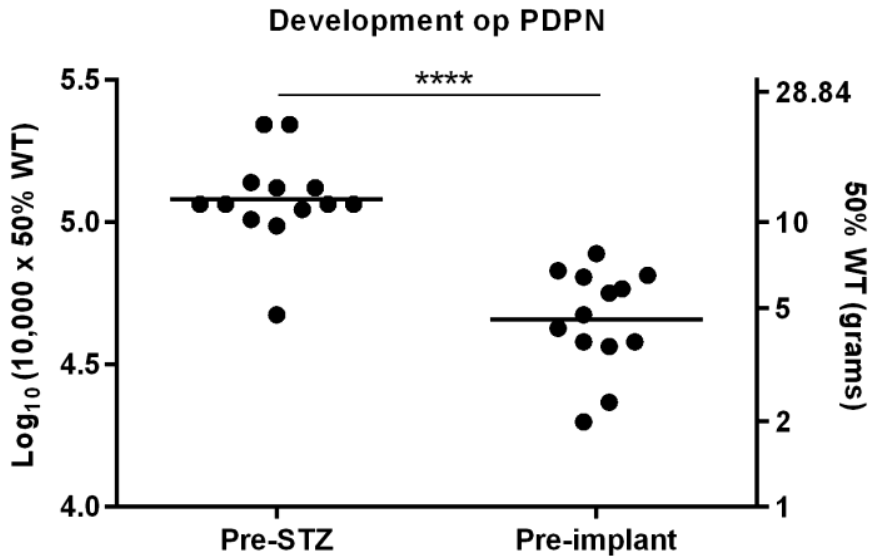

Figure 3. Development of mechanical hypersensitivity after STZ injection of all implanted rats $(\mathrm{n}=13)$. $* * * * p<$. 0.0001 compared to pre-STZ baseline.

\subsubsection{Intra-group analyses: effect of Burst-DRGS over time}

Burst-DRGS significantly attenuated STZ-induced mechanical hypersensitivity over time ( $p<0.0001$, effect of factor time; two-way repeated measures ANOVA) (Figure 4a). Burst-DRGS at 33\% MT, 50\% MT, 66\% MT, and $80 \% \mathrm{MT}$ significantly reduced mechanical hypersensitivity at $15 \mathrm{~min}, 30 \mathrm{~min}$, and $45 \mathrm{~min}$ as compared to the corresponding baseline $(p<0.05)$ (Table 1). As expected, no significant effects over time were observed for very low amplitudes of $0 \% \mathrm{MT}$ and 10\% MT (P>0.05) (Table 1).

\subsubsection{Inter-group analyses: effect between stimulation amplitudes}

A significant effect of amplitude was found $(p<0.01$, effect of factor amplitude; twoway repeated measures ANOVA) (Figure 4a). Significant differences were observed between Burst-DRGS at $33 \% \mathrm{MT}, 50 \% \mathrm{MT}, 66 \% \mathrm{MT}$ and $80 \% \mathrm{MT}$ when compared to $10 \% \mathrm{MT}$ and $0 \%$ MT ( $p<0.05$ ) (Table 1). Additionally, Burst-DRGS at 50\% MT was significantly more effective 
in normalizing STZ-induced mechanical hypersensitivity as compared to Burst-DRGS at $80 \%$ MT while the stimulator was turned on (15 and $30 \mathrm{~min})(\mathrm{p}<0.05)$ (Table 1). As expected, no significant effect of amplitude was observed in the contralateral hind paw $(p=0.69$, effect of factor amplitude; two-way repeated measures ANOVA) (Figure 4b).
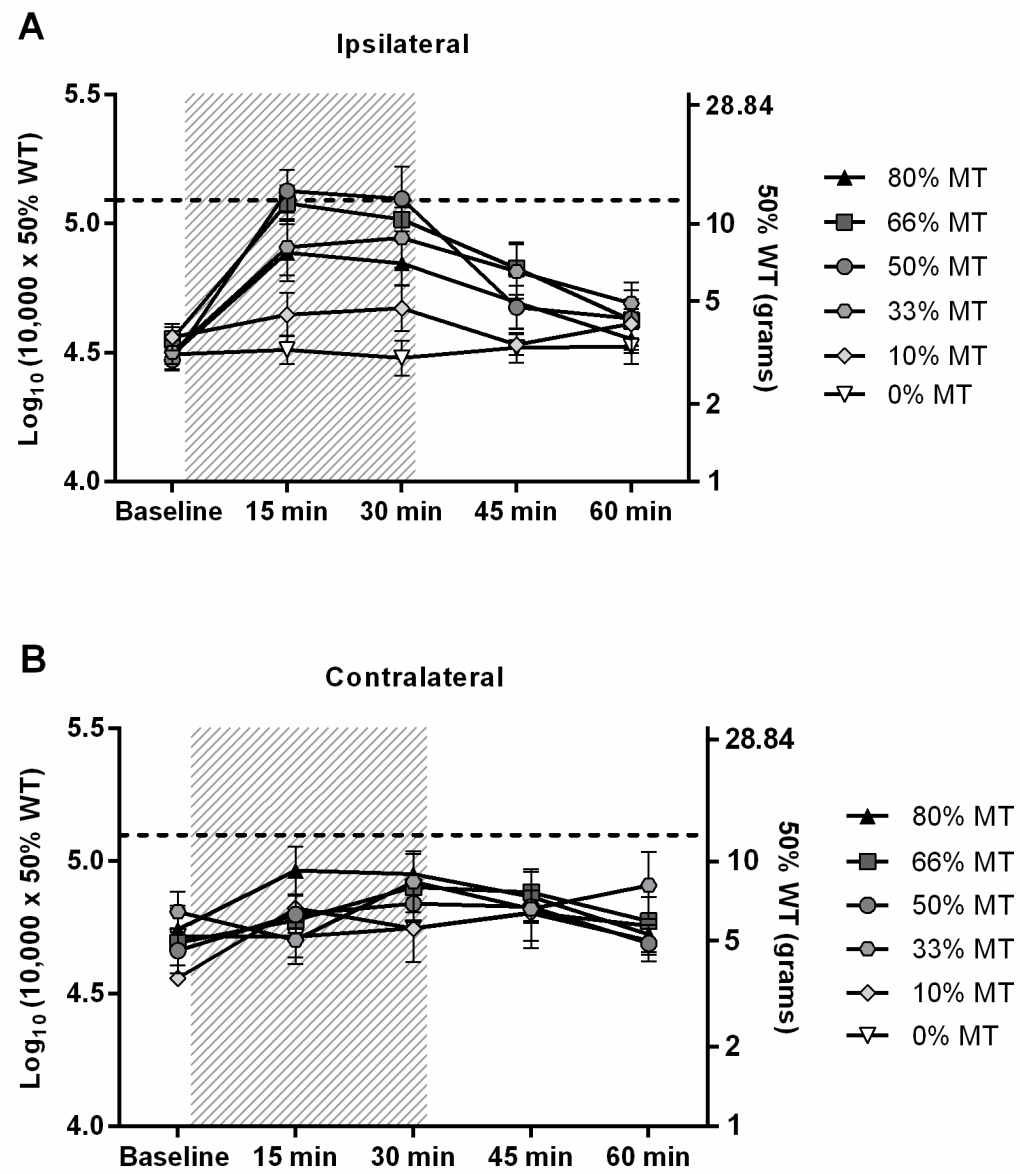

Figure 4. A combined presentation of the effect of Burst-DRGS for different amplitudes. Dotted line = the mean pre-STZ baseline of all stimulated animals. Gray area = period of DRGS. 


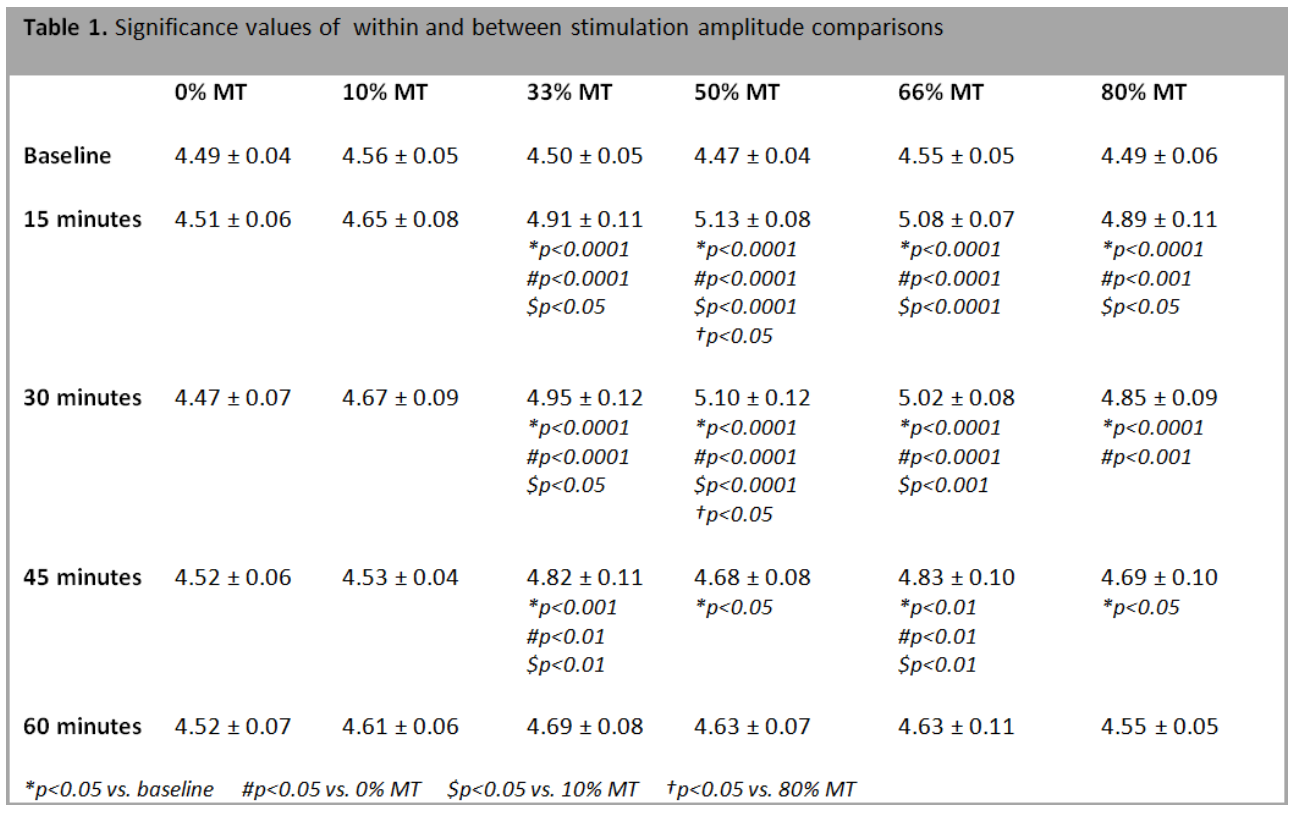

\subsubsection{Responder rates}

The percentage of animals responding to Burst-DRGS (responder defined as increase of the $\log _{10}(10,000 \times 50 \%$ WT $) \geq 0.2$ compared to pre-stimulation baseline) was highest in the $50 \% \mathrm{MT}$ and $66 \% \mathrm{MT}$ group at $15 \mathrm{~min}$ (100\%), the 66\% MT group at $30 \mathrm{~min}$ (89\%), the 66\% MT and $80 \%$ MT group at $45 \mathrm{~min}(56 \%)$, and the 50\% MT group at $60 \mathrm{~min}$ (56\%) (Table 2).

\begin{tabular}{|c|c|c|c|c|c|c|}
\hline & $0 \% \mathrm{MT}$ & $10 \% \mathrm{MT}$ & $33 \% \mathrm{MT}$ & $50 \% \mathrm{MT}$ & $66 \% \mathrm{MT}$ & $80 \% \mathrm{MT}$ \\
\hline 15 minutes & $0 / 9(0 \%)$ & $4 / 9(44 \%)$ & $7 / 9$ (78\%) & $9 / 9(100 \%)$ & $9 / 9(100 \%)$ & $6 / 9$ (67\%) \\
\hline 30 minutes & $0 / 9(0 \%)$ & $3 / 9(33 \%)$ & $7 / 9(78 \%)$ & $7 / 9$ (78\%) & $8 / 9$ (89\%) & $6 / 9(67 \%)$ \\
\hline 45 minutes & $0 / 9(0 \%)$ & $1 / 9(11 \%)$ & $4 / 9(44 \%)$ & $4 / 9(44 \%)$ & $5 / 9(56 \%)$ & $5 / 9(56 \%)$ \\
\hline 60 minutes & $1 / 9(11 \%)$ & $3 / 9(33 \%)$ & $4 / 9(44 \%)$ & $5 / 9(56 \%)$ & $2 / 9(22 \%)$ & $2 / 9(22 \%)$ \\
\hline
\end{tabular}


A

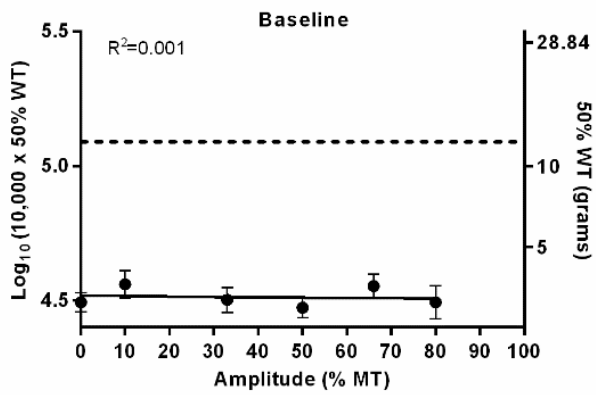

C

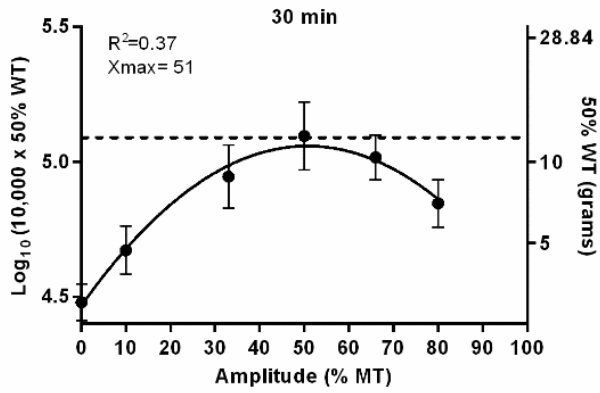

E

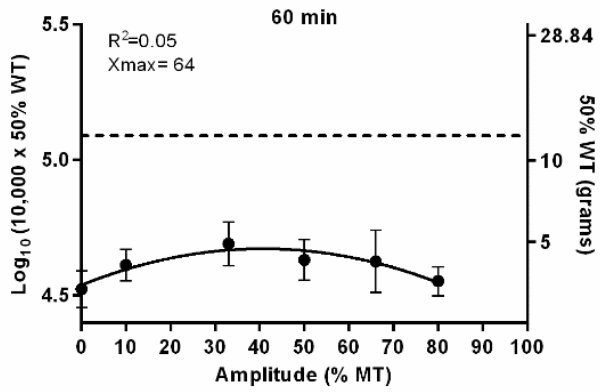

B

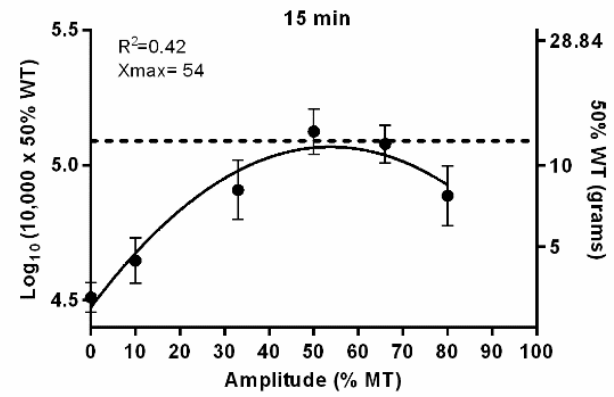

D

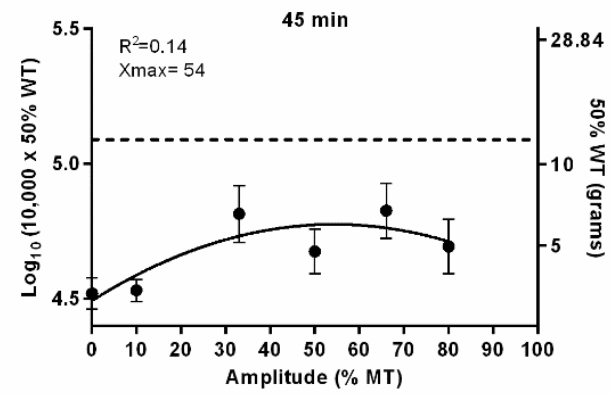

Figure 5. Plots resulting from nonlinear curve-fitting for the amplitude-mechanical hypersensitivity interaction for each time point. a. At baseline, no substantial relationship was present between amplitude and mechanical hypersensitivity. b,c. When the stimulator was turned on at 15 and $30 \mathrm{~min}$, the curves describing the amplitudemechanical hypersensitivity relationship were downward opening parabolas that can be described by the formula $y=c+b x-a x 2$. Optimal DRGS amplitudes were estimated at $51 \%-54 \%$ MT. d,e. When the stimulator was turned off at 45 and $60 \mathrm{~min}$, the nonlinear relationship became less obvious. Here, the optimal DRGS amplitude was estimated at 54\%-64\% MT. 


\subsubsection{Relationship between stimulation amplitude and mechanical hypersensitivity}

\subsubsection{Calculation of the optimal Burst-DRGS amplitude at each specific time point}

In order to investigate the relationship between amplitude and mechanical hypersensitivity, $\log _{10}(10,000 \times 50 \%$ WT) responses were expressed as a function of BurstDRGS amplitude. At pre-stimulation baseline (Figure 5a), no substantial relationship was present between amplitude and mechanical hypersensitivity $\left(R^{2}<0.001\right)$. With the stimulator turned on (15 and $30 \mathrm{~min}), \log _{10}(10,000 \times 50 \% \mathrm{WT})$ values could be expressed by a polynomial function of the second order $\left(y=c+b x-a x^{2}\right)\left(R^{2}=0.42\right.$ at $15 \mathrm{~min}, R^{2}=0.37$ at $\left.30 \mathrm{~min}\right)$. The resulting plot was parabolic in shape, with the peak of the graph (Xmax) showing the estimated maximum $\log _{10}(10,000 \times 50 \%$ WT) response and the corresponding amplitude. The calculated optimal DRGS amplitudes (Xmax) at 15 and 30 min were 54\% MT and 51\% MT, respectively (Figure $\mathbf{5 b}$-c). At 45 and $60 \mathrm{~min}$ (after the stimulator was turned off), this nonlinear relationship was less obvious $\left(R^{2}=0.14\right.$ at $45 \mathrm{~min}$ and $R^{2}=0.05$ at $\left.60 \mathrm{~min}\right)$. Optimal DRGS amplitudes were observed at an Xmax of 54\% MT and 64\% MT for 45 min and 60 min, respectively (Figure $\mathbf{5 d - e )}$.

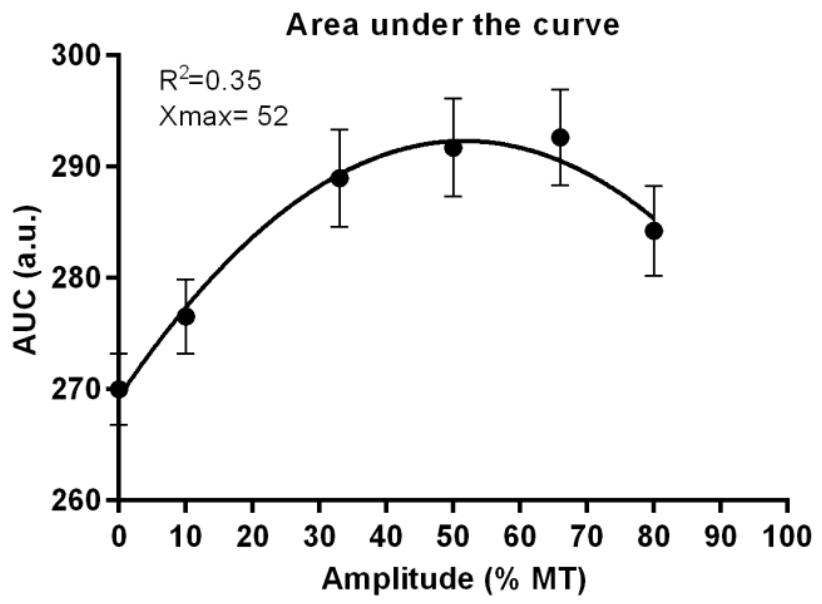

Figure 6. Plot resulting from nonlinear curve fitting for the amplitude-AUC interaction. AUCs were based on outcomes as depicted in Fig. 4a and were calculated for each animal individually before they were averaged. The resulting plot shows a clear nonlinear relationship between amplitude and AUC with an optimal DRGS amplitude at $\mathrm{Xmax}=52 \% \mathrm{MT}$. 


\subsubsection{Calculation of the overall optimal Burst-DRGS amplitude}

Also when area under the curves (AUC) from Figure 4 were plotted, the nonlinear relationship between amplitude and mechanical hypersensitivity was clearly visible $\left(R^{2}=0.35\right)$, with an estimated optimal pain relieving effect of Burst-DRGS amplitude at $X_{\max }=52 \%$ MT (Figure 6).

\subsubsection{Motor thresholds}

Lastly, MTs remained stable throughout the experiment (day 1: $0.12 \pm 0.008 \mathrm{~mA}$, day 2: $0.14 \pm 0.01 \mathrm{~mA}$, day 3: $0.18 \pm 0.03 \mathrm{~mA}$, day $4: 0.18 \pm 0.02 \mathrm{~mA}$, day 5: $0.15 \pm 0.02 \mathrm{~mA}$. $\mathrm{P}=0.07$ ) (Figure 7).

\section{Motor thresholds}

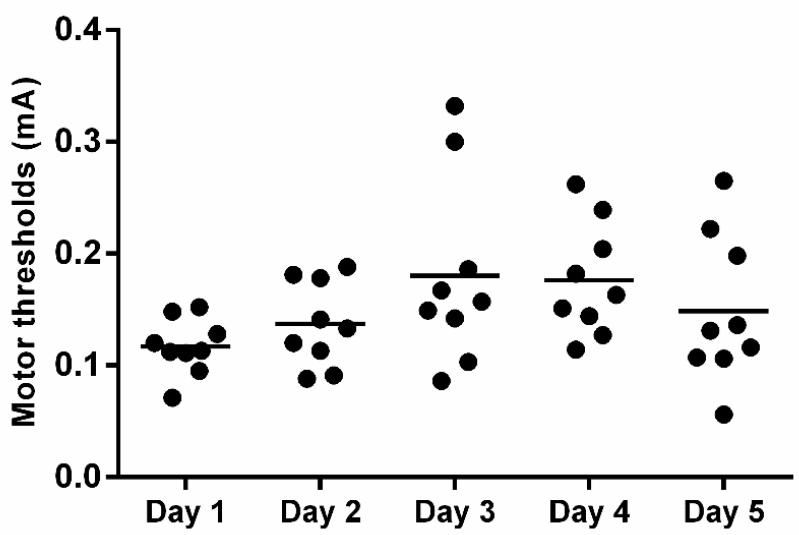

Figure 7. Observed motor thresholds (MT) throughout the study period. MT was assessed using a pulse width of $1000 \mu \mathrm{sec}$, and five pulses ( $500 \mathrm{~Hz}$ intraburst frequency) administered at an interburst frequency of $10 \mathrm{~Hz}$.

\subsection{Discussion}

The present study demonstrates that: 1 . Burst-DRGS at $33 \%-80 \%$ MT is capable of attenuating STZ-induced mechanical hypersensitivity in rats not only during stimulation, but also $15 \mathrm{~min}$ after stimulation cessation. 2. There is a nonlinear relation between Burst-DRGS amplitude and observed behavioral outcome with the best range of stimulation amplitude being $50-66 \% \mathrm{MT}$, and an estimated optimal pain relieving effect at 52\% MT. 
Burst-DRGS at amplitudes of 33\% MT, 50\% MT, 66\% MT and 80\% MT all significantly attenuated STZ-induced mechanical hypersensitivity during stimulation (15 min and $30 \mathrm{~min}$ ) as well as $15 \mathrm{~min}$ after stimulation (45 $\mathrm{min}$ ), after which mechanical hypersensitivity values returned to pre-stimulation baseline values (60 $\mathrm{min})$. These behavioral effects validate an earlier study published by our group on Burst-DRGS at $66 \% \mathrm{MT}$, which indicated that BurstDRGS is capable of treating STZ-induced mechanical hypersensitivity, with the advantage of a significant residual effect after turning off the stimulator[20]. Along these lines, results from a recent randomized controlled trial found that Burst-SCS microdosing, a paradigm that relies on the introduction of stimulation-off phases inbetween stimulation-on phases, is as effective as standard Burst-SCS, while having significantly lower battery consumption[31]. Moreover, the analgesic efficacy of Burst-SCS microdosing was found to be equal as compared to standard Burst-SCS, as measured by rat $\mathrm{FMRI}$ brain responses following noxious stimulation[32]. Together, these results strongly indicate a beneficial carry-over effect of Burst stimulation, both when delivered at the dorsal column and at the DRG, and suggest that some form of plasticity is induced following each stimulation-on phase. It has recently been shown that the amplitude of spinal neuronal responses in rats can be potentiated for several minutes following a short burst of high-frequency tetanic pulses (555 Hz)[33]. As Burst stimulation uses a similar intraburst frequency, it is reasonable to assume that Burst stimulation follows a similar mechanism, explaining the persistence of pain relief when the stimulator is off (stimulation-off phase). Furthermore, high frequency stimulation is known to induce long-term potentiation in lamina I of spinal projection neurons[34], and signs of short term plasticity have been found in response to electrical stimulation, including those modulated by Burst stimulation[35].

Previous studies have indicated that Burst stimulation can be optimized by adjusting relevant stimulation parameters to modulate the charge delivered to the spinal cord during stimulation, such as amplitude[22]. In our study, there seems to be no linear correlation between Burst-DRGS amplitude and the observed behavioral outcome, as the highest amplitude $(80 \% \mathrm{MT})$ did not result in optimal pain relief and highest responder rates. Significant differences were observed between 33\% MT, 50\% MT, and 66\% MT (at 15 min, 30 
min and $45 \mathrm{~min}$ ) when compared to 10\% MT and 0\% MT, and Burst-DRGS at 50\% MT was significantly more effective in normalizing STZ-induced mechanical hypersensitivity as compared to Burst-DRGS at $80 \%$ MT while the stimulator was turned on (15 and $30 \mathrm{~min}$ ). Moreover, Burst-DRGS at 50\% MT and 66\% MT (15 min) and Burst-DRGS at 66\% MT (30 min) yielded the highest responder rates, seemingly favoring amplitudes of $50-66 \%$ over the higher (80\% MT) and lower (0-33\% MT) stimulation amplitudes. Lastly, the estimated optimal amplitude of DRGS based on nonlinear regression of AUCs was found to be $52 \%$ MT. Interestingly, previous work on high frequency SCS suggests the sensation threshold, defined as the amplitude at which animals start to show signs of disturbance of their normal behavior, to be around 50\% MT [36]. The gradual decrease in therapy efficacy with stimulation amplitudes beyond this optimum might be explained by the fact that sensory, potential uncomfortable, sensations induced by the stimulation might have taken place. Interestingly, a recent study by Meuwissen et al. showed that Burst-SCS also followed this nonlinear course between amplitude and behavioral outcome [23]. The optimal amplitude for Burst-SCS in that study was reported to be $50 \% \mathrm{MT}$, and the behavioral outcome was shown to decline rather rapidly when this optimal amplitude was surpassed[23]. This is in line with the findings of Courtney et al. who demonstrated that the absolute therapeutic window of Burst-SCS in terms of amplitude is considerably smaller when compared to Con-SCS [37]. In contrast, the Burst-DRGS paradigm as used in the present study demonstrated a larger optimal therapeutic window (50-67\% MT).Differences in terms of this optimal therapeutic window between our study and the study of Meuwissen and colleagues might be attributed to the experimental model used (PDPN vs. Seltzer lesion), the location of stimulation (spinal cord vs. DRG), the type of stimulation used (quadripolar vs. bipolar), and/or the Burst waveform used (biphasic with active recharge balance vs. monophasic with passive recharge balance) [23]. Additionally, a study by Tang et al. found that while spinal neuronal responses to colorectal distension and pinch were reduced similarly using tonic SCS and Burst-SCS at $90 \%$ MT, Burst, but not tonic SCS significantly decreased the nociceptive somatic response after colorectal distension or pinch using lower amplitudes of $60 \% \mathrm{MT}[21]$. Combined, these data suggest that Burst stimulation is effective at lower amplitudes relative to the observed MT as 
compared to tonic stimulation. The combination of this relatively low optimal Burst stimulation amplitude, both in DRGS and SCS studies, together with the earlier described increased carry-over effect of Burst stimulation, could have important implications for optimal stimulation delivery as well as battery life of the IPG in clinical practice.

To date, few studies have investigated the mechanisms underlying (Burst-)DRGS and the question remains how (Burst-)DRGS might affect the nociceptive afferents and firing of these afferents. The DRG consists of a unique pseudounipolar design, in which the T-junction of the DRG can act as a selective filter for stimuli i.e. action potentials traveling towards the spinal cord[38]. A study by Koopmeiners et al. reported DRGS to reduce the amplitude and/or amount of action potentials arising from the DRG, thereby inhibiting neuronal excitability [39]. Interestingly, it was recently suggested that the outcome of dorsal column SCS is correlated to the active stimulation period or duty cycle[40], and that SCS can attenuate aberrant, hyperactive firing of pain transmission neurons[40,41]. Along these lines, a stimulation mode with a high duty cycle, like Burst-SCS, but also Burst-DRGS, is more likely to counteract this aberrant firing of pain transmission neurons. The impact of the high duty cycle of Burst-DRGS may therefore preclude the strength-duration and charge-duration relationship from defining the relationship between single pulse parameters and neural activation thresholds. Besides these electrophysiological findings, DRGS has been linked to attenuation of brain area's that are considered to be part of the pain matrix like the contralateral thalamic VPL/VPM nuclei, and cortical S1 and S2[42], and one can argue that also spinal mechanisms may underlie DRGS as modulating firing rates of DRG neurons by DRGS may also affect interneurons and GABAergic systems in the dorsal horn as is the case in traditional SCS[43-45]. However, more research is needed to fully understand the underlying mechanisms of DRGS and its relation to specific waveforms, like Burst-DRGS and/or individual DRGS-stimulation parameters, like amplitude.

Limitations of this study include the time frame of the used stimulation protocol. In clinical practice, patients typically receive DRGS for a longer period of time as compared to the short-term protocol used in this study. Second, it should be stressed that using the MT 
for determining the desired stimulation amplitude in rodents may have several shortcomings. In preclinical studies, the MT often replaces the perception threshold (PT) as used in humans, since it is a quick, easy to use, and objective outcome measure based on visible contractions of the lower trunk and/or hind limbs. In contrast, the PT is relatively difficult to observe in rodents, as the experimenter has to 'determine' whether or not an animal perceives the stimulation or not, which can lead to subjectivity. The PT has been estimated to be approximately $30-50 \% \mathrm{MT}$ in rats[36, 46], and a study by Koyama et al. found that this PT/MT ratio varies across rats[46]. The latter might interfere with direct translation of our results to the clinic, as there might have been differences in the amount of stimulation relative to the PT (\% PT) delivered to the system across animals. However, it is worth noting that the MTs in the study of Koyama and colleagues were measured under anesthesia, which might significantly influence the (variation between) observed MTs in their study[46]. We are convinced that future studies should try to objectively establish the PT in rodent models which would then allow for an even more precise translation of findings to the human situation. Additionally, it should also be noted that there might be differences in the exact MT amplitude when stimulating with an interburst frequency of $10 \mathrm{~Hz}$ (which was used to determine the MT used in the experiment) as compared to an interburst frequency of $40 \mathrm{~Hz}$ (which was used for final delivery of Burst-DRGS), given the lower duty cycle of $10 \mathrm{~Hz}$ stimulation. As it is near impossible to objectively assess MTs using higher frequencies, including $40 \mathrm{~Hz}$, preclinical studies tend to use lower frequencies for assessment of MT (both in DRGS and (Burst-)SCS studies). The latter is needed to obtain a clear and easy to observe $M T$, even though final delivery of stimulation happens at higher frequencies $[23,47,48]$. Third, in the present study we chose to only include female Sprague-Dawley rats. Female rats reach their mature body weight and nerve conduction status faster as compared to their male counterpart, or either sex of other strains [24]. As such, one should be cautious when extrapolating these results to the male sex [49]. Fourth, there are fundamental differences in the clinical and preclinical manifestation of PDPN. In patients, diabetes is a chronic disease where complications often only arise after many years. Streptozotocin injection in rats relies on destruction of beta cells in the pancreas[24]. The injection leads therefore to a very swift 
development of diabetes (within one week), and subsequent development of mechanical allodynia (within 4 weeks) $[20,27,29,50]$. Although the injection of streptozotocin is the most common used diabetes model in rats, one should be cautious when extrapolating these results to a human situation. Lastly, the results presented here are limited to reflex-based outcome measures (Von Frey) in experimental PDPN. Future studies using spontaneous or operant behavioral tests and/or different animal models should be conducted to verify these results, as it is very well possible that Burst stimulation only targets specific components of pain, not observable by the Von Frey test[51].

Altogether, our findings indicate that there is a nonlinear relationship between Burst-DRGS amplitude and behavioral outcome, as Burst-DRGS at 50\% MT and 66\% MT resulted in optimal pain relief and highest responder rates, with an estimated optimal pain relief at 52\% MT. A further increase in Burst-DRGS amplitude up to $80 \% \mathrm{MT}$ did not result in better pain relief, even though Burst-DRGS at amplitudes of 33\% MT-80\% MT significantly attenuated mechanical hypersensitivity in PDPN animals. Also, a significant wash-out of Burst-DRGS was observed 15 min following Burst-DRGS cessation at these amplitudes. Further optimization and analysis of DRGS driven by insights into the underlying mechanisms related to the various stimulation paradigms is warranted. 


\subsection{References}

1. Abbott CA, Malik RA, van Ross ER, Kulkarni J, Boulton AJ. Prevalence and characteristics of painful diabetic neuropathy in a large community-based diabetic population in the U.K. Diabetes Care. 2011;34(10):2220-4.

2. Daousi C, MacFarlane IA, Woodward A, Nurmikko TJ, Bundred PE, Benbow SJ. Chronic painful peripheral neuropathy in an urban community: a controlled comparison of people with and without diabetes. Diabet Med. 2004;21(9):976-82.

3. Davies M, Brophy S, Williams R, Taylor A. The prevalence, severity, and impact of painful diabetic peripheral neuropathy in type 2 diabetes. Diabetes Care. 2006;29(7):1518-22.

4. Kaur S, Pandhi P, Dutta P. Painful diabetic neuropathy: an update. Ann Neurosci. 2011;18(4):168-75.

5. Finnerup NB, Otto M, Jensen TS, Sindrup SH. An evidence-based algorithm for the treatment of neuropathic pain. MedGenMed. 2007;9(2):36.

6. de Vos CC, Meier K, Zaalberg PB, Nijhuis HJ, Duyvendak W, Vesper J, et al. Spinal cord stimulation in patients with painful diabetic neuropathy: a multicentre randomized clinical trial. Pain. 2014;155(11):2426-31.

7. Slangen R, Schaper NC, Faber CG, Joosten EA, Dirksen CD, van Dongen RT, et al. Spinal cord stimulation and pain relief in painful diabetic peripheral neuropathy: a prospective two-center randomized controlled trial. Diabetes Care. 2014;37(11):3016-24.

8. van Beek M, Slangen R, Schaper NC, Faber CG, Joosten EA, Dirksen CD, et al. Sustained Treatment Effect of Spinal Cord Stimulation in Painful Diabetic Peripheral Neuropathy: 24-Month Follow-up of a Prospective TwoCenter Randomized Controlled Trial. Diabetes Care. 2015;38(9):e132-4.

9. Liem L, Russo M, Huygen FJ, Van Buyten JP, Smet I, Verrills P, et al. A multicenter, prospective trial to assess the safety and performance of the spinal modulation dorsal root ganglion neurostimulator system in the treatment of chronic pain. Neuromodulation. 2013;16(5):471-82; discussion 82.

10. Liem L, Russo M, Huygen FJ, Van Buyten JP, Smet I, Verrills P, et al. One-year outcomes of spinal cord stimulation of the dorsal root ganglion in the treatment of chronic neuropathic pain. Neuromodulation. 2015;18(1):41-8; discussion 8-9.

11. Kramer J, Liem L, Russo M, Smet I, Van Buyten JP, Huygen F. Lack of body positional effects on paresthesias when stimulating the dorsal root ganglion (DRG) in the treatment of chronic pain. Neuromodulation. 2015;18(1):50-7; discussion 7.

12. Huygen F, Liem L, Cusack W, Kramer J. Stimulation of the L2-L3 Dorsal Root Ganglia Induces Effective Pain Relief in the Low Back. Pain Pract. 2018;18(2):205-13.

13. Deer TR, Levy RM, Kramer J, Poree L, Amirdelfan K, Grigsby E, et al. Dorsal root ganglion stimulation yielded higher treatment success rate for complex regional pain syndrome and causalgia at 3 and 12 months: a randomized comparative trial. Pain. 2017;158(4):669-81.

14. Bayman EO, Brennan TJ. Incidence and severity of chronic pain at 3 and 6 months after thoracotomy: meta-analysis. J Pain. 2014;15(9):887-97.

15. Eldabe S, Burger K, Moser H, Klase D, Schu S, Wahlstedt A, et al. Dorsal Root Ganglion (DRG) Stimulation in the Treatment of Phantom Limb Pain (PLP). Neuromodulation. 2015;18(7):610-6; discussion 6-7.

16. Eldabe S, Espinet A, Wahlstedt A, Kang P, Liem L, Patel NK, et al. Retrospective Case Series on the Treatment of Painful Diabetic Peripheral Neuropathy With Dorsal Root Ganglion Stimulation. Neuromodulation. 2018.

17. De Ridder D, Vanneste S, Plazier M, van der Loo E, Menovsky T. Burst spinal cord stimulation: toward paresthesia-free pain suppression. Neurosurgery. 2010;66(5):986-90.

18. De Ridder D, Plazier M, Kamerling N, Menovsky T, Vanneste S. Burst spinal cord stimulation for limb and back pain. World Neurosurg. 2013;80(5):642-9 e1.

19. De Ridder D, Lenders MW, De Vos CC, Dijkstra-Scholten C, Wolters R, Vancamp T, et al. A 2-center comparative study on tonic versus burst spinal cord stimulation: amount of responders and amount of pain suppression. Clin J Pain. 2015;31(5):433-7.

20. Franken G, Debets J, Joosten EAJ. Dorsal Root Ganglion Stimulation in Experimental Painful Diabetic Peripheral Neuropathy: Burst vs. Conventional Stimulation Paradigm. Neuromodulation. 2018.

21. Tang R, Martinez M, Goodman-Keiser M, Farber JP, Qin C, Foreman RD. Comparison of burst and tonic spinal cord stimulation on spinal neural processing in an animal model. Neuromodulation. 2014;17(2):143-51.

22. Crosby ND, Goodman Keiser MD, Smith JR, Zeeman ME, Winkelstein BA. Stimulation parameters define the effectiveness of burst spinal cord stimulation in a rat model of neuropathic pain. Neuromodulation. 2015;18(1):1-8; discussion 
23. Meuwissen KPV, Gu JW, Zhang TC, Joosten EAJ. Conventional-SCS vs. Burst-SCS and the Behavioral Effect on Mechanical Hypersensitivity in a Rat Model of Chronic Neuropathic Pain: Effect of Amplitude. Neuromodulation. 2018;21(1):19-30.

24. Calcutt NA. Modeling diabetic sensory neuropathy in rats. Methods Mol Med. 2004;99:55-65.

25. Chaplan SR, Bach FW, Pogrel JW, Chung JM, Yaksh TL. Quantitative assessment of tactile allodynia in the rat paw. J Neurosci Methods. 1994;53(1):55-63.

26. Mills C, Leblond D, Joshi S, Zhu C, Hsieh G, Jacobson P, et al. Estimating efficacy and drug ED50's using von Frey thresholds: impact of weber's law and log transformation. J Pain. 2012;13(6):519-23.

27. Pluijms WA, van Kleef M, Honig WM, Janssen SP, Joosten EA. The effect of spinal cord stimulation frequency in experimental painful diabetic polyneuropathy. Eur J Pain. 2013;17(9):1338-46.

28. van Beek M, Hermes D, Honig WM, Linderoth B, van Kuijk SMJ, van Kleef M, et al. Long-Term Spinal Cord Stimulation Alleviates Mechanical Hypersensitivity and Increases Peripheral Cutaneous Blood Perfusion in Experimental Painful Diabetic Polyneuropathy. Neuromodulation. 2018.

29. van Beek M, van Kleef M, Linderoth B, van Kuijk SM, Honig WM, Joosten EA. Spinal cord stimulation in experimental chronic painful diabetic polyneuropathy: Delayed effect of High-frequency stimulation. Eur J Pain. 2017;21(5):795-803.

30. Pan B, Yu H, Fischer GJ, Kramer JM, Hogan QH. Dorsal Root Ganglionic Field Stimulation Relieves Spontaneous and Induced Neuropathic Pain in Rats. J Pain. 2016;17(12):1349-58.

31. Vesper J, Slotty P, Schu S, Poeggel-Kraemer K, Littges H, Van Looy P, et al. Burst SCS Microdosing Is as Efficacious as Standard Burst SCS in Treating Chronic Back and Leg Pain: Results From a Randomized Controlled Trial. Neuromodulation. 2019;22(2):190-3.

32. Saber M SD, Tessmer J, Khan Z, Kent A, Robinson M, Hogan Q, Pawela C. Rat fMRI brain responses to noxious stimulation during tonic, burst, and burst-microdosing spinal cord stimulation. Poster, International Neuromodulation Society 2019.

33. Lloyd DP. Post-tetanic potentiation of response in monosynaptic reflex pathways of the spinal cord. J Gen Physiol. 1949;33(2):147-70.

34. Ikeda H, Heinke B, Ruscheweyh R, Sandkuhler J. Synaptic plasticity in spinal lamina I projection neurons that mediate hyperalgesia. Science. 2003;299(5610):1237-40.

35. Shyu BC, Vogt BA. Short-term synaptic plasticity in the nociceptive thalamic-anterior cingulate pathway. Mol Pain. 2009;5:51.

36. Song Z, Viisanen H, Meyerson BA, Pertovaara A, Linderoth B. Efficacy of kilohertz-frequency and conventional spinal cord stimulation in rat models of different pain conditions. Neuromodulation. 2014;17(3):22634; discussion 34-5.

37. Courtney P, Espinet A, Mitchell B, Russo M, Muir A, Verrills P, et al. Improved Pain Relief With Burst Spinal Cord Stimulation for Two Weeks in Patients Using Tonic Stimulation: Results From a Small Clinical Study. Neuromodulation. 2015;18(5):361-6.

38. Krames ES. The dorsal root ganglion in chronic pain and as a target for neuromodulation: a review. Neuromodulation. 2015;18(1):24-32; discussion

39. Koopmeiners AS, Mueller S, Kramer J, Hogan QH. Effect of electrical field stimulation on dorsal root ganglion neuronal function. Neuromodulation. 2013;16(4):304-11; discussion 10-1.

40. Miller JP, Eldabe S, Buchser E, Johanek LM, Guan Y, Linderoth B. Parameters of Spinal Cord Stimulation and Their Role in Electrical Charge Delivery: A Review. Neuromodulation. 2016;19(4):373-84.

41. Elbasiouny SM, Mushahwar VK. Suppressing the excitability of spinal motoneurons by extracellularly applied electrical fields: insights from computer simulations. J Appl Physiol (1985). 2007;103(5):1824-36.

42. Pawela CP, Kramer JM, Hogan QH. Dorsal root ganglion stimulation attenuates the BOLD signal response to noxious sensory input in specific brain regions: Insights into a possible mechanism for analgesia. Neuroimage. 2017;147:10-8.

43. Stiller CO, Cui JG, O'Connor WT, Brodin E, Meyerson BA, Linderoth B. Release of gamma-aminobutyric acid in the dorsal horn and suppression of tactile allodynia by spinal cord stimulation in mononeuropathic rats. Neurosurgery. 1996;39(2):367-74; discussion 74-5.

44. Cui JG, O'Connor WT, Ungerstedt U, Linderoth B, Meyerson BA. Spinal cord stimulation attenuates augmented dorsal horn release of excitatory amino acids in mononeuropathy via a GABAergic mechanism. Pain. 1997;73(1):87-95. 
45. Janssen SP, Gerard S, Raijmakers ME, Truin M, Van Kleef M, Joosten EA. Decreased intracellular GABA levels contribute to spinal cord stimulation-induced analgesia in rats suffering from painful peripheral neuropathy: the role of KCC2 and GABA(A) receptor-mediated inhibition. Neurochem Int. 2012;60(1):21-30.

46. Koyama S, Xia J, Leblanc BW, Gu JW, Saab CY. Sub-paresthesia spinal cord stimulation reverses thermal hyperalgesia and modulates low frequency EEG in a rat model of neuropathic pain. Sci Rep. 2018;8(1):7181.

47. Pan B, Yu H, Fischer GJ, Kramer JM, Hogan QH. Dorsal Root Ganglionic Field Stimulation Relieves Spontaneous and Induced Neuropathic Pain in Rats. J Pain. 2016.

48. Meuwissen KPV, Gu JW, Zhang TC, Joosten EAJ. Burst Spinal Cord Stimulation in Peripherally Injured Chronic Neuropathic Rats: A Delayed Effect. Pain Pract. 2018.

49. Craft RM, Mogil JS, Aloisi AM. Sex differences in pain and analgesia: the role of gonadal hormones. Eur J Pain. 2004;8(5):397-411.

50. Koetsier E, Franken G, Debets J, van Kuijk SMJ, Perez R, Linderoth B, et al. Effectiveness of dorsal root ganglion stimulation and dorsal column spinal cord stimulation in a model of experimental painful diabetic polyneuropathy. CNS Neurosci Ther. 2018.

51. Meuwissen KPV, van Beek M, Joosten EAJ. Burst and Tonic Spinal Cord Stimulation in the Mechanical Conflict-Avoidance System: Cognitive-Motivational Aspects. Neuromodulation. 2019. 


\section{CHAPTER}

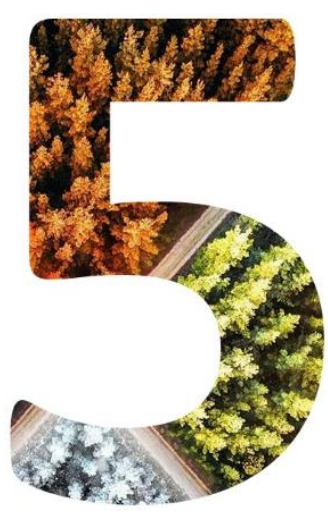

\section{A Possible Mechanism of Conventional}

Dorsal Root Ganglion Stimulation in Pain

Relief: Quantitative Immunocytochemical

Analysis of Intracellular GABA in DRG

\section{Neurons}

G. Franken, P. Douven, J. Debets, Hellen Steinbusch, E.A. Joosten

Submitted. 


\subsection{Abstract}

Objectives: The sensory cell somata in the DRG contain all necessary equipment for extensive GABAergic signaling, and are able to release GABA upon depolarization. With this study we hypothesized that pain relief induced by conventional dorsal root ganglion stimulation (Con-DRGS) in animals with experimental painful diabetic peripheral neuropathy is related to the release of GABA from DRG neurons. With use of quantitative immunocytochemistry we hypothesize DRGS to result in a decreased intensity of intracellular GABA-immunostaining in DRG somata.

Methods: Female Sprague-Dawley rats $(n=31)$ were injected with streptozotocin (STZ) in order to induce Diabetes Mellitus (DM). Only animals that developed neuropathic pain after 4 weeks (Von Frey) were implanted with a unilateral, DRGS device L4 ( $n=14)$. Animals were then stimulated for 30 minutes, and received either Con-DRGS $(20 \mathrm{~Hz}$, pulse width $=0.2 \mathrm{~ms}$, amplitude $=67 \%$ of motor threshold, $\mathrm{n}=8$ ) or Sham-DRGS (amplitude set at zero, $n=6$ ) while pain behavior (von Frey) was measured. After 30 minutes, animals were immediately perfused, after which the ipsi- and contralateral DRG's were collected. DRG's were processed and sectioned, after which sections were immunostained for GABA. Quantitative immunocytochemical analysis was performed and the intensity of staining was measured based on gray values per cell. Relation with size of sensory cell soma diameter (small: 12-26 $\mu \mathrm{m}$, assumed to be C-fiber related sensory neurons; medium: 26-40 $\mu \mathrm{m}$, assumed to be $A \delta$ related sensory neurons; and large: $40-54 \mu \mathrm{m}$, assumed to be $A \beta$ related sensory neurons) was included.

Results: DRGS treated animals showed significant reductions in STZ-induced mechanical hypersensitivity. No significant differences in GABA immunostaining intensity per sensory neuron cell soma type (small, medium, or large-sized) were noted in DRG's of stimulated (Con-DRGS) animals versus Sham animals. No differences in GABA immunostaining intensity per sensory cell soma type in ipsi- as compared to contralateral DRG's were observed. 
Conclusion: Con-DRGS does not affect the average intracellular GABA immunofluorescence staining intensity in DRG sensory neurons of those animals which showed significant pain reduction. Similarly, no soma size related changes in intracellular GABA immunofluorescence were observed following Con-DRGS.

Keywords: GABA, dorsal root ganglion stimulation, neuromodulation, painful diabetic peripheral neuropathy, neuropathic pain 


\subsection{Introduction}

Spinal cord stimulation (SCS) is a last-resort treatment option for patients with intractable chronic neuropathic pain [1, 2]. SCS, first utilized in 1967 by Shealy and colleagues [3], emerged as a direct spin-off of the two years earlier published "Gate-control" theory by Melzack and Wall [4]. According to the Gate Control Theory, antidromic stimulation of the non-nociceptive $A \beta$-fibers located in the dorsal column of the spinal cord can close the "spinal gate" by blocking incoming nociceptive signals which enter the spinal cord from the

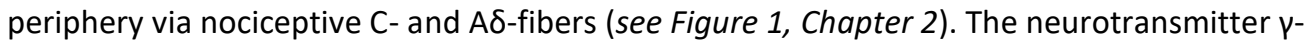
aminobutyric acid (GABA) is thought to be a key molecule in this process [5-7]. A study by Janssen et al. found intracellular GABA levels to be increased in the dorsal horn of the spinal cord following peripheral nerve injury [8]. Later, it was shown that conventional (Con-)SCS decreases the intracellular GABA concentration in the dorsal horn of the spinal cord in this same neuropathic pain model [9]. At the same time, multiple studies have shown that extracellular GABA levels, as measured by microdialysis, are increased following Con-SCS [1012]. Also the application of a $\mathrm{GABA}_{\mathrm{B}}$ receptor antagonist can transiently abolish the SCSinduced effects in neuropathic rats [10], and application of sub-effective doses of Baclofen (a $\mathrm{GABA}_{B}$ receptor agonist) was shown to turn SCS non-responders into SCS responders both in rats [13] as well as humans [14]. Thus, spinal GABA release seems to play a pivotal role in the analgesic mechanism of action (MoA) of Con-SCS of the dorsal columns.

Dorsal root ganglion stimulation (DRGS) is a promising novel addition to the field of SCS $[15,16]$ (see Chapter 2), with advantages over SCS in terms of battery consumption, area coverage, and for some indications, such as complex regional pain syndrome (CRPS), efficacy [17]. Over the years, an increasing amount of literature has been published on the analgesic properties of DRGS, including both clinical [18] and rodent studies [19-24]. Whereas SCS is thought to only recruit $A \beta$-fibers, DRGS can theoretically stimulate not only $A \beta$-, but also $C$ and A $\delta$-fibers due to the unique properties of the DRG [25]. The somata of all peripheral fibers ( $A \beta-, C$ - and $A \delta$-fibers) reside in the DRG, and the DRG has shown to be surprisingly tolerant for trauma following lead insertion [26]. These different types of sensory DRG neurons have different properties, both in terms of electrophysiological responses as well as 
morphology. Whereas the touch-affiliated $A \beta$-fibers have thick, myelinated fibers with a large cell soma $(40-54 \mu \mathrm{m})$ and high conduction velocity, the nociceptive A $\delta$-fibers (thinly myelinated, medium-sized cell soma: $26-40 \mu \mathrm{m}$, medium conduction velocity) and C-fibers (unmyelinated, small-sized cell soma: 12-26 $\mu \mathrm{m}$, low conduction velocity) have their own distinct electrophysiological and morphological properties [27]. To date, the MoA of DRGS remains largely unknown. It was recently shown that DRGS with conventional settings (ConDRGS) does not decrease intracellular levels of GABA immunoreactive staining in the dorsal horn of the spinal cord in rats with painful diabetic polyneuropathy (PDPN) [28]. From this, it is concluded that Con-DRGS, in contrast to Con-SCS, is not likely to depend on GABA release in the dorsal horn of the spinal cord. In this context it is interesting that Du et al. reported the presence of an extensive GABAergic communication network between sensory neuron somata inside the DRG itself [29]. The authors showed that sensory neurons in the DRG express major proteins required for GABA synthesis and release, and that these neurons are capable of releasing GABA upon depolarization. From this it was postulated that the GABAergic system in the DRG itself may act as a second gate, in addition to the aforementioned gate control theory (or first gate) in the spinal dorsal horn.

In the present study, we therefore hypothesized that electrical stimulation of the DRG with conventional settings (Con-DRGS) decreases intracellular GABA immunoreactivity in DRG somata of those animals which showed reduced mechanical hypersensitivity. To investigate this, we quantitatively assessed the intensity of local intracellular GABA immunoreactivity in the L4 DRG of Con-DRGS or sham treated PDPN animals.

\subsection{Methods}

\subsubsection{Animals}

This study was performed in 31 female Sprague Dawley rats (Charles River, Maastricht, Netherlands), with an average body weight of 160-220 g at the start of the experiment. Animals were socially housed in a climate-controlled room at a 12/12 reversed day-night cycle in transparent polycarbonate cages, with ad libitum access to food and drinking water. The experiments described in this study were approved by the Animal Care 
Committee of the Maastricht University Medical Centre (under project license 2017-022), and experiments were performed in accordance with the guidelines of the European Directive for the Protection of Vertebrate Animals Used for Experimental and Other Scientific Purposes (86/609/EU).

\subsubsection{Induction of Diabetes Mellitus (DM)}

Before streptozotocin (STZ) injections, animals were weighed and fasted overnight. $65 \mathrm{mg} / \mathrm{kg}$ freshly dissolved STZ in $0.9 \% \mathrm{NaCl}$ was then intraperitoneally injected to induce Diabetes Mellitus (DM). Six days following STZ injection, blood glucose levels of the animals were measured using an Accu-Chek Aviva ${ }^{\circledR}$ glucometer (Roche Diagnostics GmbH, Mannheim, Germany). Only rats with a blood glucose level of $\geq 15 \mathrm{mmol} / \mathrm{L}$ were considered diabetic and included in the study [30-34].

\subsubsection{Assessment of mechanical hypersensitivity}

Pain behavior was assessed by applying Von Frey monofilaments (bending forces $0.6,1.2,2.0,3.6,5.5,8.5,15.1$, and $28.84 \mathrm{~g}$ ) to the plantar surface of the hind paws of the animals at baseline (before STZ injection) and 4 weeks following STZ injection. Rats were individually placed in plastic cages with a mesh floor, after which they were allowed to habituate to the testing environment for 15 minutes. The $50 \%$ withdrawal threshold (WT) was then calculated based on the up-down method as previously described [35]. A cut-off of $28.84 \mathrm{~g}$ was used in order to prevent tissue damage. Calculated $50 \%$ WT values were then multiplied by 10.000 and logarithmically transformed to obtain a linear scale and account for Weber's law [36]. Only animals with a decrease of $\geq 0.2$ of the $\log 10(10000 \times 50 \%$ WT $)$ unit were considered to have mechanical hypersensitivity and were treated with dorsal root ganglion stimulation (DRGS).

\subsubsection{Dorsal root ganglion stimulation (DRGS)}

A bipolar, custom-made DRGS lead was implanted as previously described [19-22, 26]. Briefly, a paravertebral incision was made, and the intravertebral foramen of $L 4$ was exposed. The foramen was then opened, and both the anode and cathode of the electrode 
were inserted into the foramen in order to stimulate the L4 DRG. The electrode was then secured to the transverse process caudal to the $L 4$ foramen using a small screw and steel wire. The external connectors of the electrode were then tunneled under the skin via the neck of the animal, and the incision was closed in layers.

Animals were externally stimulated for 30 minutes with Con-DRGS $(20 \mathrm{~Hz}, 0.2 \mathrm{~ms}$ pulse width, amplitude at $67 \%$ of the motor threshold (MT)) or Sham-DRGS (amplitude at 0\% MT) using a Proclaim implantable pulse generator (IPG) (Abbott Inc., Texas, USA) in the first week following DRGS implantation [30,31]. The MT was measured before stimulation onset using a frequency of $2 \mathrm{~Hz}$, and pulse width of $0.2 \mathrm{~ms}$. The MT was defined as the current inducing contractions of the lower trunk or hind limb. In order to test the effect of Con-DRGS on pain behavior, Von Frey measurements were performed just before, and at 30 minutes following onset of Con-DRGS. Following 30 minutes of DRGS, animals were immediately anesthetized with pentobarbital $(100 \mathrm{mg} / \mathrm{kg})$ and transcardially perfused with $15 \%$ picric acid and $4 \%$ paraformaldehyde in $0.2 \mathrm{M}$ phosphate buffer saline (PBS; $\mathrm{pH} 7.6$ ).

\subsubsection{Tissue preparation}

Following transcardial perfusion, both the ipsi- and contralateral L4 DRG were extracted as previously described [37]. Tissue was then stored in PBS with 1\% azide until further use. DRG's were pre-embedded in a $50: 50$ mix of $4 \%$ agar $/ 5 \%$ gelatin in order to prevent tissue shrinking, followed by paraffin embedding. Tissue was serially sectioned using a microtome at a thickness of $4 \mu \mathrm{m}$, after which each out of 10 sections were mounted on glass slides (each $40 \mu \mathrm{m}$ ). The glass slides containing the tissue were then incubated overnight at a temperature of $37^{\circ} \mathrm{C}$, after which they were stored at room temperature until further use.

\subsubsection{Immunohistochemical GABA staining}

Sections were first deparaffinized using a sequence of 2x 15 min xylene, 2x 5 min $100 \%$ ethanol, $2 \times 5 \min 96 \%$ ethanol, and 2x 70\% ethanol. Slides were then washed $10 \mathrm{~min}$ with Tris-buffered saline (TBS, $0.1 \mathrm{M}, \mathrm{pH} 7.6$ ) including 0.3\% Triton X-100 (TBS-T), $10 \mathrm{~min}$ TBS, and $10 \mathrm{~min}$ TBS-T. Next, sections were blocked for $1 \mathrm{~h}$ using $2 \%$ normal donkey serum (Sigma- 
Aldrich, Zwijndrecht, The Netherlands, D9663) diluted in TBS-T. Slides were then incubated overnight with a rabbit anti-GABA polyclonal antibody (1:1000 diluted in TBS-T; SigmaAldrich, Zwijndrecht, The Netherlands, A2052). After rinsing unbound primary antibody in TBS (3x10 min), slides were incubated with the far-red secondary antibody Alexa-Fluor 647 donkey anti-rabbit IgG (1:100 diluted in TBS-T; Invitrogen, Breda, The Netherlands) for $2 \mathrm{~h}$. Slides were then again washed in TBS ( $3 \times 10 \mathrm{~min}$ ), after which slides were incubated with Hoechst (1:1000 in TBS) for 15 minutes in order to visualize nuclei. Lastly, slides were washed $3 \times 10 \mathrm{~min}$ in TBS, and then coverslipped with TBS/glycerol (80\%/20\%).

\subsubsection{Quantitative immunocytochemical analysis}

Following the GABA-staining protocol, immunostained sections were observed under a Disk Scanning Unit (DSU) microscope (Olympus, Tokyo, Japan). First, photomicrographs were taken of the DRG sections (3 sections per DRG) using Micromanager Software (Ron Vale's Laboratory, UCSF, San Francisco, USA). Images were then merged together using Adobe Photoshop (Adobe Inc., San Jose, USA), and somata were outlined. Only somata with a visible nucleus were included in the analysis, which, in combination with the fact that only one section in every $40 \mu \mathrm{m}(1: 10)$ was mounted on a glass slide, should make sure that no cells were measured twice. Average grayscale values at the 647 channel were analyzed per cell soma using ImageJ software (National Institutes of Health (NIH) and the Laboratory for Optical and Computational Instrumentation (LOCI), University of Wisconsin, USA), and the corresponding diameter per soma was measured and noted. Averages gray values per treatment group were then calculated, as well as the average gray values per cell diameter per treatment group. 3 diameter ranges were chosen based on literature: 12-26 $\mu \mathrm{m}$ (small-sized soma, assumed to be C-fiber related sensory neurons)), 26$40 \mu \mathrm{m}$ (medium-sized soma, assumed to be A $\delta$ related sensory neurons), and 50-54 $\mu \mathrm{m}$ (large-sized soma, assumed to be $A \beta$ related sensory neurons) [27]. The investigator was blinded for the condition of the tissue and animals throughout the whole experiment. 


\subsubsection{Statistical analysis}

Data are presented as mean \pm standard error of the mean (SEM). For statistical analysis, Von Frey data were logarithmically transformed to account for Weber's Law and obtain a linear scale [36]. For comparisons between pre-STZ WTs and pre-implant WTs, and analysis of the effect of DRGS on WTs, a one-way analysis of variance (ANOVA) was performed, followed by Tukey's multiple comparison test. For the comparison of MTs between groups, an unpaired t-test was used. For comparisons of grayscale values between treatments (DRGS vs. Sham-DRGS), and between ipsilateral and contralateral, one-way and two-way ANOVA's were used, followed by a Tukey's multiple comparisons test. A P value < 0.05 was considered statistically significant. All statistical analysis was performed using GraphPad Prism software version 8.4.3 (GraphPad Software Inc., California, USA).

\subsection{Results}

\subsubsection{Flowchart of animals}

Twenty-six out of 31 rats that were injected with STZ developed DM within one week (84\%; blood glucose level $>15 \mathrm{mmol} / \mathrm{L}$ ). No animals required additional insulin treatment (blood glucose $\geq 31.4 \mathrm{mmol} / \mathrm{L}$ ). Out of the 26 diabetic rats, 14 animals developed PDPN after 4 weeks (54\%; $\geq 0.2$ decrease in $\log _{10}(10,000 \times 50 \%$ WT) on von Frey when compared to the pre-STZ injection baseline), and were subsequently implanted with a unilateral DRGS device at the L4 lumbar level in week 5. The remaining 12 rats were excluded from the study and sacrificed.

\subsubsection{Behavior}

\subsubsection{Development of PDPN}

The mean $\log _{10}(10,000 \times 50 \%$ WT) of the 14 implanted animals dropped from $5.3 \pm$ $0.05 \mathrm{~g}$ (ipsilateral hind paw) and $5.1 \pm 0.05 \mathrm{~g}$ (contralateral hind paw) at pre-STZ-baseline to $4.8 \pm 0.06 \mathrm{~g}$ (ipsilateral hind paw; $\mathrm{p}<0.0001$ compared to baseline) and $4.8 \pm 0.07 \mathrm{~g}$ (contralateral hind paw; $\mathrm{p}<0.01$ compared to baseline) at pre-implantation ( 4 weeks after STZ injection). No differences between the ipsi- and contralateral hind paw were noted at 
either the pre-STZ baseline $(p=0.20)$ or the pre-implantation measurement $(p>0.99)$ (Figure 1A).

\subsubsection{Dorsal root ganglion stimulation}

No significant differences in terms of MT's were observed between the Con-DRGS and Sham-DRGS group, indicating correct and stable implantation of the DRGS lead in both arms (DRGS: $202 \pm 79 \mu \mathrm{A}$, Sham-DRGS: $236 \pm 39 \mu \mathrm{A} ; \mathrm{p}=0.74$ ) (Figure 1B).

Con-DRGS significantly attenuated STZ-induced mechanical hypersensitivity in the ipsilateral (stimulated) hind paw. 6/8 animals (75\%) were classified as responders to ConDRGS $\left(\geq 0.2\right.$ increase in $\log _{10}(10,000 \times 50 \%$ WT) on Von Frey when compared to the stim off measurement). Average ipsilateral $\log _{10}(10.000 \times 50 \% \mathrm{WT})$ values were $4.8 \pm 0.10 \mathrm{~g}$ at baseline (before Con-DRGS onset), and significantly increased to $5.2 \pm 0.07 \mathrm{~g}$ after $30 \mathrm{~min}$ of Con-DRGS $(p<0.05)$. Contralateral $\log _{10}(10.000 \times 50 \%$ WT) values also increased from $4.9 \pm$ $0.10 \mathrm{~g}$ at baseline (before Con-DRGS onset) to $5.1 \pm 0.09 \mathrm{~g}$ after $30 \mathrm{~min}$ of Con-DRGS, albeit not significant $(p>0.05)$. No significant differences were observed between the ipsi-and contralateral hind paw at either baseline (stim off; $p=0.27$ ) or the 30 min time point (stim on; $\mathrm{p}=0.75$ ) (Figure 1C).

No effect of Sham-DRGS was observed on either the ipsilateral or contralateral hind paw. Average ipsilateral $\log _{10}(10.000 \times 50 \%$ WT) values were $4.9 \pm 0.09 \mathrm{~g}$ at baseline (before Sham-DRGS onset), and $4.8 \pm 0.08 \mathrm{~g}$ after $30 \mathrm{~min}$ of Sham-DRGS $(\mathrm{p}=0.11)$. Contralateral $\log _{10}$ $(10.000 \times 50 \%$ WT) values were $5.0 \pm 0.11$ at baseline (before Sham-DRGS onset), and $4.9 \pm$ $0.12 \mathrm{~g}$ after $30 \mathrm{~min}$ of Sham-DRGS $(p=0.25)$. No significant differences were observed between the ipsi-and contralateral hind paw at either baseline (stim off; $p=0.69$ ) or the 30 min time point (stim on; $p=0.44$ ) (Figure 1D). 

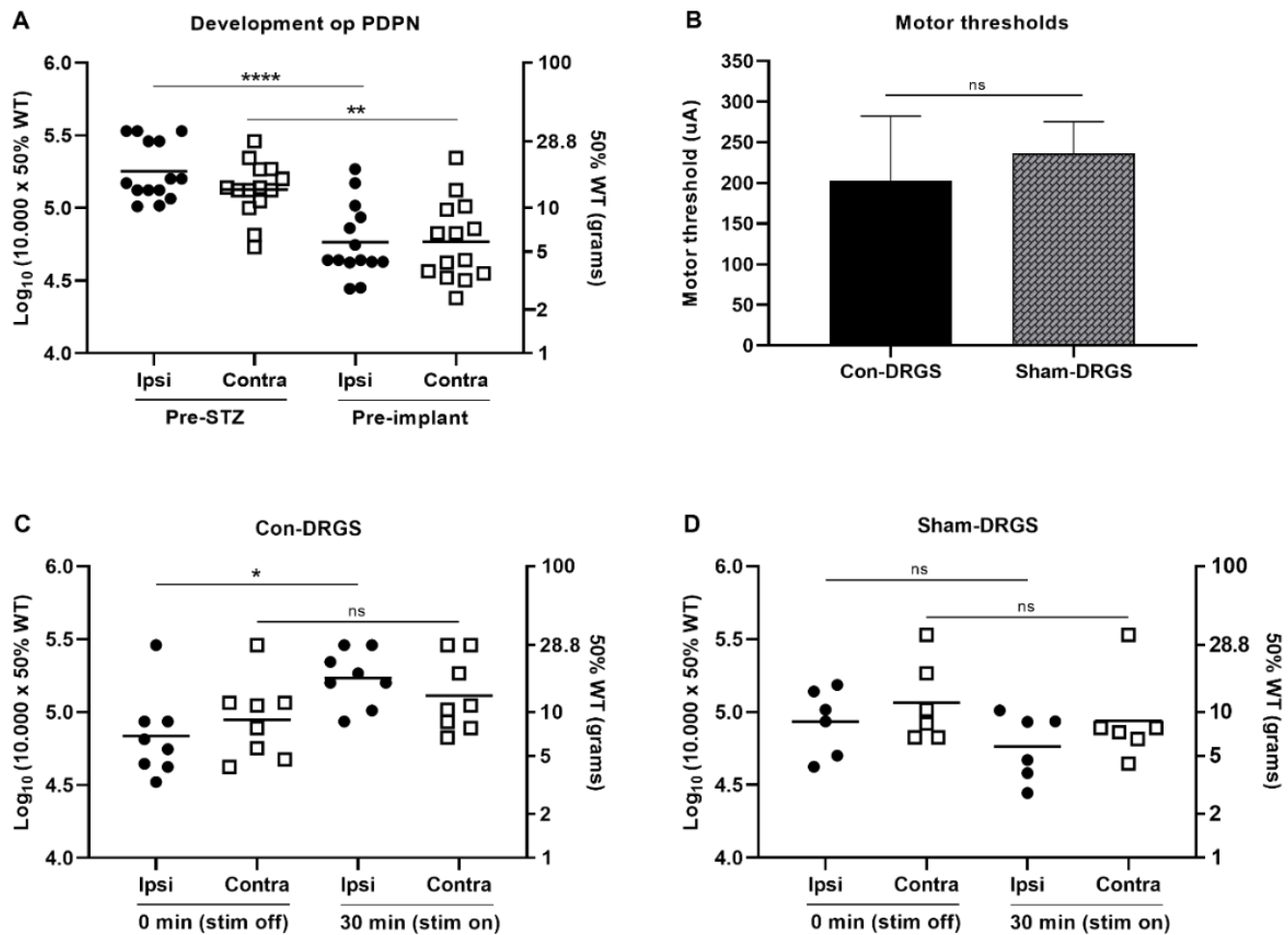

Figure 1. (A) Development PDPN over time in all implanted rats $(n=14)$. Animals developed profound STZ-induced mechanical hypersensitivity in both the ipsilateral and contralateral hind paw (B). No significant differences were observed in terms of average MT's between the Con-DRGS and Sham-DRGS group. MT was assessed using a frequency of $2 \mathrm{~Hz}$, and pulse width of $0.2 \mathrm{~ms}$. (C) The effect of $30 \mathrm{~min}$ of Con-DRGS $(20 \mathrm{~Hz})$ on mechanical hypersensitivity values of both the ipsilateral (stimulated) and contralateral (unstimulated) hind paw $(n=8)$. ConDRGS attenuated STZ-induced mechanical hypersensitivity in the ipsilateral, but not contralateral hind paw. (D) No significant effect was observed of Sham-DRGS on mechanical hypersensitivity values of both the ipsi- and contralateral hind paw $(\mathrm{n}=6) .{ }^{*} \mathrm{p}<0.05 ;{ }^{*} \mathrm{p}<0.01 ; * * * \mathrm{p}<0.0001 ; \mathrm{ns}=$ not significant. 


\subsubsection{Immunohistochemical GABA staining}

\subsubsection{Average gray values}

Anti-GABA immunostained sections showed a strong and specific GABA-IR (Figure 2A). No significant intragroup differences (ipsi vs. contra) in terms of average gray values were observed in either the Con-DRGS $(p=0.99)$ or Sham-DRGS group $(p=0.71)$. Along the same lines, no significant intergroup differences (Con-DRGS vs. Sham-DRGS) were observed in neither the ipsilateral DRG $(p=0.99)$ nor the contralateral DRG $(p=0.64)$ (Figure 2B).

\subsubsection{Average gray values per soma diameter}

We then tested whether there was a difference in the average gray value of small(12-26 $\mu \mathrm{m})$, medium- (26-40 $\mu \mathrm{m})$, and large- $(40-54 \mu \mathrm{m})$ sized somata, and whether or not Con-DRGS specifically acts on one of these ranges of soma diameter. An overall significant effect of diameter was noted $(p<0.0001)$. A significant difference was also noted between the gray values of the small vs. medium (Con-DRGS ipsi; $p<0.01$, Con-DRGS contra; $p<0.05$, Sham-DRGS contra; $p<0.05$ ) and small vs. large sized somata (Sham-DRGS contra; $p<0.05$ ). A trend was also observed between the medium vs. large sized somata in the Sham-DRGS contra DRG sections ( $p=0.09)$ (Figure $2 \mathrm{C}$ ).

No intragroup differences (ipsi vs. contra) in terms of gray values were observed in both the Con-DRGS and Sham-DRGS group for all three soma diameter ranges (Con-DRGS: small; $p=0.99$, medium; $p=0.99$, large; $p=0.99$. Sham-DRGS: small; $p=0.44$, medium; $p=0.46$, large; $p=0.79$ ). Along these lines, no intergroup differences (Con-DRGS vs. Sham-DRGS) in terms of gray values were observed in the ipsilateral and contralateral DRG for all soma diameter ranges (ipsilateral: small; $p=0.99$, medium; $p=0.99$, large; $p=0.99$. Contralateral: small; $p=0.53$, medium; $p=0.61$, large; $p=0.95$ ) (Figure $2 \mathrm{C}$ ). 

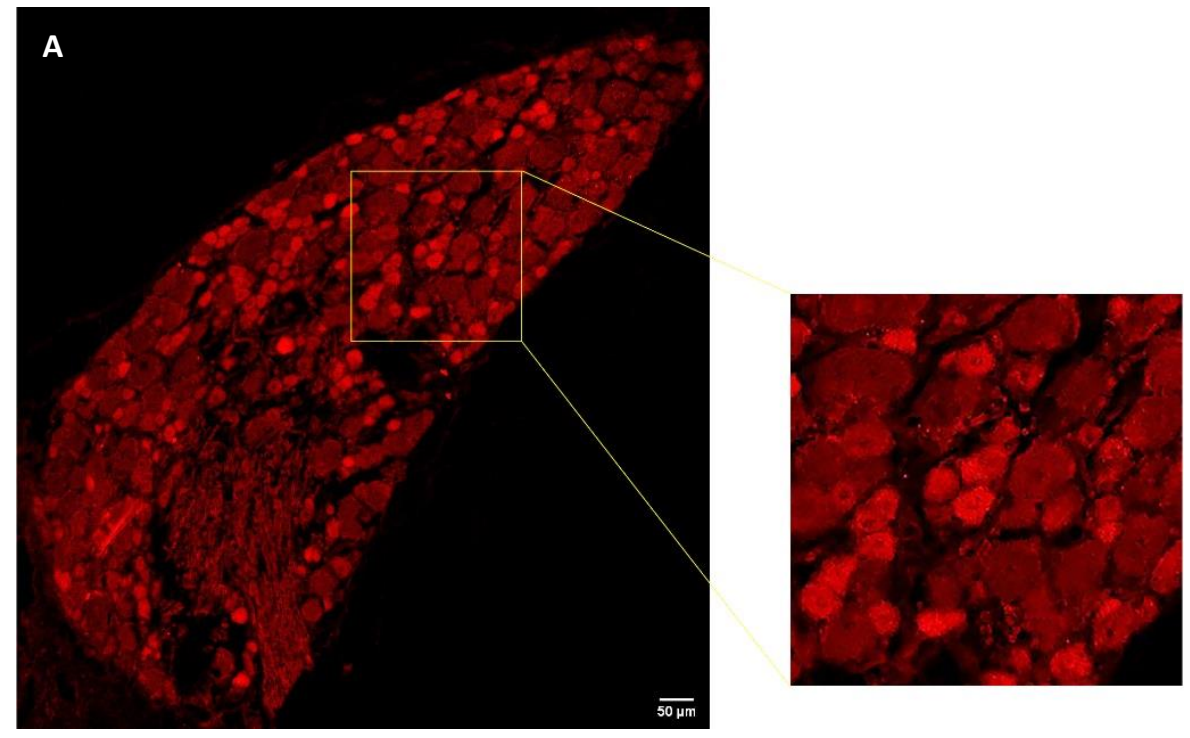

B

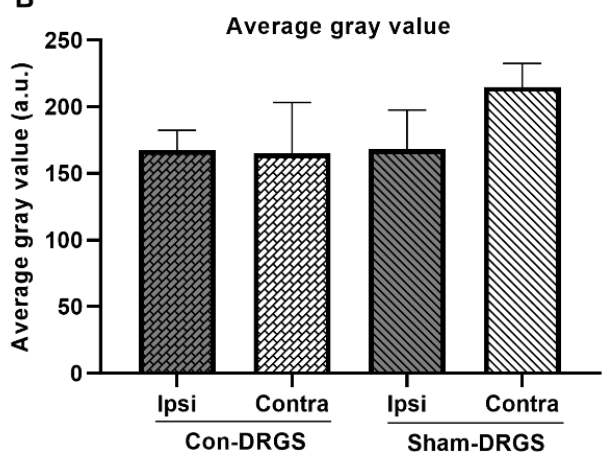

C

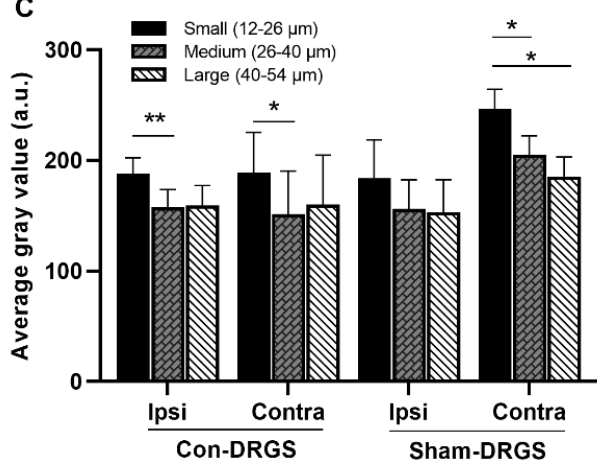

Figure 2. (A) Representative photomicrograph of anti-GABA immunostaining. Note the difference in staining intensity between the large and small diameter somata. (B) Comparison of average gray values in the ipsi- and contralateral DRG of both the Con-DRGS and Sham-DRGS treated group. No differences were observed between ipsi- and contralateral DRG's in both the Con-DRGS and Sham-DRGS group, nor were differences observed between the Con-DRGS and Sham-DRGS group in both the ipsi- and contralateral DRG. (C) Comparison of average gray values based on soma diameter. Significant differences were observed in intensity of GABA-immunoreactivity between the small as compared to the medium and large sized soma. No differences were observed between ipsiand contralateral DRG's in both the Con-DRGS and Sham-DRGS group for all three soma diameter ranges, nor were differences observed between the Con-DRGS and Sham-DRGS group in both the ipsi- and contralateral DRG for all three soma diameter ranges. ${ }^{*} \mathrm{p}<0.05 ;{ }^{*} \mathrm{p}<0.01$. 


\subsection{Discussion}

The present study showed that Con-DRGS does not alter the average intracellular GABA immunofluorescence staining intensity in DRG sensory neurons. Similarly, no cell size specific effect was noted as the intensity of GABA-immunoreactivity in cell somata of small (12-26 $\mu \mathrm{m})$, medium (26-40 $\mu \mathrm{m})$ or large DRG sensory neurons (40-54 $\mu \mathrm{m})$ was similar between Con-DRGS and Sham-DRGS treated animals. At the same time, animals did show marked reductions in STZ-induced mechanical hypersensitivity values after 30 min of stimulation. These reductions in pain behavior are comparable to those obtained previously in the same diabetic rat model $[20,30,31,38]$, as well as in rat models of tibial nerve injury (TNI) [26, 39], collagen-induced rheumatoid arthritis [23], and intra-articular knee monosodium iodoacetate-induced osteoarthritis [39].

The role of GABA as a key player in the mechanism underlying Con-SCS of the dorsal columns has been well established over the years. The general consensus is that, in accordance with the Gate Control Theory [4], Con-SCS antidromically stimulates the nonnociceptive $A \beta$-fibers, and thereby releases GABA from inhibitory interneurons in the dorsal horn of the spinal cord. This is represented by a decrease in intracellular GABA immunoreactivity in the dorsal horn of the spinal cord directly following stimulation $[9,40]$, whereas extracellular levels of GABA, as measured by microdialysis, rise [10-12]. Moreover, the analgesic properties of Con-SCS can be blocked by the application of GABA antagonists $[10,40]$, while they can be improved by the application of GABAB receptor agonists $[13,14]$.

In theory, this same principle may hold true also for DRGS. As all the somata of the $A \beta$-fibers reside in the DRG, one could speculate that DRGS activates these $A \beta$-fibers, and thereby induces the release of GABA in the spinal cord dorsal horn, similarly to what happens in Con-SCS of the dorsal columns. Indeed, a computational analysis performed by Graham and colleagues found Con-DRGS to modulate A $\beta$-fiber, but not C-fiber activity [41], which could theoretically lead to the occurrence of pain gating mechanisms in the spinal cord dorsal horn. However, a study by Koetsier et al., using the same PDPN model as used in the present 
study, found that Con-DRGS does not affect the intensity of GABA-immunoreactivity staining in the dorsal spinal horn, and from this concluded that Con-DRGS is not likely to release GABA in the dorsal horn of the spinal cord [42]. It is based on these observations suggested that it is unlikely that Con-DRGS relies on GABAergic pain gating mechanisms in the spinal cord dorsal horn.

Con-DRGS differentiates itself from Con-SCS by having access to the soma of all three types of sensory fibers: $A \delta, A \beta$, and C-type soma. Along these lines, another computational study found that DRGS in fact does modulate C-fiber activity, and that DRGS can amplify local T-junction filtering, thereby blocking afferent signaling [43]. The latter finding is consistent with multiple electrophysiological studies $[25,44]$, and might indicate that a more local effect is responsible for the analgesic properties of DRGS.

A recent study by Du and colleagues reported the presence of an extensive, local, GABAergic network in the DRG itself, as DRG cells expressed all the major proteins required for GABA synthesis and release [29]. They also reported that DRG neurons of all sizes (small DRG neurons assumed to be C-fiber related; medium DRG neurons assumed to be $A \delta$ related; large DRG neurons assumed to be $A \beta$ related) are capable of releasing GABA upon depolarization, and that GABA can produce a net inhibitory effect at the T-junction in small diameter cells [29]. Based on these findings, we hypothesized that Con-DRGS induces the release of GABA from DRG somata for its analgesic action and thus that Con-DRGS would result in decreased intensity of intracellular GABA-immunoreactivity. Clearly, we were not able to demonstrate any differences between Con-DRGS treated animals and Sham-DRGS treated animals in terms of average GABA immunofluorescence, nor were differences observed between the ipsilateral and contralateral DRG of (Sham-)DRGS treated animals. Furthermore, as it is possible that specific soma types behave differently in response to ConDRGS, something that is impossible to detect by only measuring the average immunofluorescence per condition, we also looked into the effect of Con-DRGS on somata classified based on three ranges of diameter: 12-26 $\mu \mathrm{m}, 26-40 \mu \mathrm{m}$, and 40-54 $\mu \mathrm{m}$ [27]. In general, the small and medium-sized soma are more likely to include nociceptive cells (C-type 
and A $\delta$-type respectively), whereas the large-sized soma are more likely to include nonnociceptive cells (A $\beta$-type). In this context, especially the small type, nociceptive DRG somata (12-26 $\mu \mathrm{m})$ were found to express significantly more GABA as compared to the medium and large sized soma in most groups, suggesting the importance of GABA in (slow) nociceptive signaling. Interestingly, the medium sized nociceptive cells $(26-40 \mu \mathrm{m}$; assumed to be Aठ somata) showed the same GABA staining intensity as the non-nociceptive $A \beta$ somata. Nevertheless, no significant effects were observed between Con-DRGS and Sham-DRGS for all three types of DRGS soma (small, medium, large), nor were ipsi- contralateral differences observed. This indicates that Con-DRGS does not act by reducing intracellular GABA concentrations in neither nociceptive (small and medium sized somata) nor non-nociceptive cells (large sized somata).

Although it is tempting to strictly classify the small, medium, and large cells as Ctype soma, A $\delta$-type soma, and $A \beta$-type soma, respectively, one should be cautious in doing so, as $C, A \delta$, and $A \beta$-type in rat DRG cells are known to superpose the soma diameter [27]. Furthermore, Lee et al. showed that the soma size of these intermediate cells is not correlated with conduction velocity because these cells appear both with myelinated as well as unmyelinated axons [45]. Moreover, the GABA immunoreactivity was analyzed in a 2D plane, which makes it possible that some somata that were classified as being medium or even small-sized, might in fact be larger cells due to the unknown depth of the Z-axis. Future studies might therefore be undertaken including double labeling of GABA with $C_{-}, \mathrm{A} \delta$-, and/or $A \beta$-specific antibodies, as well as 3D visualizations of DRG cell somata.

From these results it seems very well possible that Con-DRGS acts via totally different, GABA independent, mechanisms. As mentioned earlier, there is some evidence suggesting the ability of DRGS to inhibit the T-junction electrophysiologically [25, 44]. Furthermore, an $\mathrm{fMRI}$ study showed that DRGS can attenuate BOLD signals in pain-affiliated brain regions, such as the contralateral thalamic VPL/VPM nuclei, and cortical S1 and S2 [46]. Lastly, it was recently suggested that DRGS, especially at low frequencies $(<20 \mathrm{~Hz})$, might induce dorsal horn inhibition via the activation of low threshold mechanoreceptors (LTMR's) 
and the activation the body's own opioid system via the release of endorphins and dynorphins [47].

In conclusion, under the conditions tested, we found no evidence for a local, GABAmediated MoA of Con-DRGS and suggest a GABA independent mechanism to be involved in pain relief. The future research agenda should include more specific classification of nociceptive and non-nociceptive neurons in the DRG, as well as 3D visualizations of the DRG cell somata. 


\subsection{References}

1. Medical Advisory S. Spinal cord stimulation for neuropathic pain: an evidence-based analysis. Ont Health Technol Assess Ser. 2005;5(4):1-78.

2. Kumar K, Abbas M, Rizvi S. The use of spinal cord stimulation in pain management. Pain Manag. 2012;2(2):125-34.

3. Shealy CN, Mortimer JT, Reswick JB. Electrical inhibition of pain by stimulation of the dorsal columns: preliminary clinical report. Anesth Analg. 1967;46(4):489-91.

4. Melzack R, Wall PD. Pain mechanisms: a new theory. Science. 1965;150(3699):971-9.

5. Vallejo R, Bradley K, Kapural L. Spinal Cord Stimulation in Chronic Pain: Mode of Action. Spine (Phila Pa 1976). 2017;42 Suppl 14:S53-S60.

6. Guan Y. Spinal cord stimulation: neurophysiological and neurochemical mechanisms of action. Curr Pain Headache Rep. 2012;16(3):217-25.

7. Sdrulla AD, Guan Y, Raja SN. Spinal Cord Stimulation: Clinical Efficacy and Potential Mechanisms. Pain Pract. 2018;18(8):1048-67.

8. Janssen SP, Truin M, Van Kleef $M$, Joosten EA. Differential GABAergic disinhibition during the development of painful peripheral neuropathy. Neuroscience. 2011;184:183-94.

9. Janssen SP, Gerard S, Raijmakers ME, Truin M, Van Kleef M, Joosten EA. Decreased intracellular GABA levels contribute to spinal cord stimulation-induced analgesia in rats suffering from painful peripheral neuropathy: the role of KCC2 and GABA(A) receptor-mediated inhibition. Neurochem Int. 2012;60(1):21-30.

10. Cui JG, O'Connor WT, Ungerstedt U, Linderoth B, Meyerson BA. Spinal cord stimulation attenuates augmented dorsal horn release of excitatory amino acids in mononeuropathy via a GABAergic mechanism. Pain. 1997;73(1):87-95.

11. Stiller CO, Cui JG, O'Connor WT, Brodin E, Meyerson BA, Linderoth B. Release of gamma-aminobutyric acid in the dorsal horn and suppression of tactile allodynia by spinal cord stimulation in mononeuropathic rats. Neurosurgery. 1996;39(2):367-74; discussion 74-5.

12. Linderoth B, Stiller CO, Gunasekera L, O'Connor WT, Ungerstedt U, Brodin E. Gamma-aminobutyric acid is released in the dorsal horn by electrical spinal cord stimulation: an in vivo microdialysis study in the rat. Neurosurgery. 1994;34(3):484-8; discussion 8-9.

13. Cui JG, Meyerson BA, Sollevi A, Linderoth B. Effect of spinal cord stimulation on tactile hypersensitivity in mononeuropathic rats is potentiated by simultaneous $G A B A(B)$ and adenosine receptor activation. Neurosci Lett. 1998;247(2-3):183-6.

14. Lind G, Schechtmann G, Winter J, Meyerson BA, Linderoth B. Baclofen-enhanced spinal cord stimulation and intrathecal baclofen alone for neuropathic pain: Long-term outcome of a pilot study. Eur J Pain. 2008;12(1):1326.

15. Krames ES. The dorsal root ganglion in chronic pain and as a target for neuromodulation: a review. Neuromodulation. 2015;18(1):24-32; discussion

16. Liem L, van Dongen E, Huygen FJ, Staats P, Kramer J. The Dorsal Root Ganglion as a Therapeutic Target for Chronic Pain. Reg Anesth Pain Med. 2016;41(4):511-9.

17. Deer TR, Levy RM, Kramer J, Poree L, Amirdelfan K, Grigsby E, et al. Dorsal root ganglion stimulation yielded higher treatment success rate for complex regional pain syndrome and causalgia at 3 and 12 months: a randomized comparative trial. Pain. 2017;158(4):669-81.

18. Vuka I, Marcius T, Dosenovic S, Ferhatovic Hamzic L, Vucic K, Sapunar D, et al. Neuromodulation with electrical field stimulation of dorsal root ganglion in various pain syndromes: a systematic review with focus on participant selection. J Pain Res. 2019;12:803-30.

19. Koetsier E, Franken G, Debets J, van Kuijk SMJ, Perez R, Linderoth B, et al. Effectiveness of dorsal root ganglion stimulation and dorsal column spinal cord stimulation in a model of experimental painful diabetic polyneuropathy. CNS Neurosci Ther. 2019;25(3):367-74.

20. Koetsier E, Franken G, Debets J, van Kuijk SMJ, Linderoth B, Joosten EA, et al. Dorsal Root Ganglion Stimulation in Experimental Painful Diabetic Polyneuropathy: Delayed Wash-Out of Pain Relief After Low-Frequency $(1 \mathrm{~Hz})$ Stimulation. Neuromodulation. 2020;23(2):177-84.

21. Franken G, Debets J, Joosten EAJ. Nonlinear Relation Between Burst Dorsal Root Ganglion Stimulation Amplitude and Behavioral Outcome in an Experimental Model of Painful Diabetic Peripheral Neuropathy. Neuromodulation. 2020;23(2):158-66. 
22. Franken G, Debets J, Joosten EAJ. Dorsal Root Ganglion Stimulation in Experimental Painful Diabetic Peripheral Neuropathy: Burst vs. Conventional Stimulation Paradigm. Neuromodulation. 2019;22(8):943-50.

23. Pan B, Zhang Z, Chao D, Hogan QH. Dorsal Root Ganglion Field Stimulation Prevents Inflammation and Joint Damage in a Rat Model of Rheumatoid Arthritis. Neuromodulation. 2018;21(3):247-53.

24. Yu G, Segel I, Zhang Z, Hogan QH, Pan B. Dorsal Root Ganglion Stimulation Alleviates Pain-related Behaviors in Rats with Nerve Injury and Osteoarthritis. Anesthesiology. 2020.

25. Koopmeiners AS, Mueller S, Kramer J, Hogan QH. Effect of electrical field stimulation on dorsal root ganglion neuronal function. Neuromodulation. 2013;16(4):304-11; discussion 10-1.

26. Pan B, Yu H, Fischer GJ, Kramer JM, Hogan QH. Dorsal Root Ganglionic Field Stimulation Relieves Spontaneous and Induced Neuropathic Pain in Rats. J Pain. 2016;17(12):1349-58.

27. Villiere V, McLachlan EM. Electrophysiological properties of neurons in intact rat dorsal root ganglia classified by conduction velocity and action potential duration. J Neurophysiol. 1996;76(3):1924-41.

28. Koetsier E, Franken G, Debets J, Heijmans L, van Kuijk SMJ, Linderoth B, et al. Mechanism of dorsal root ganglion stimulation for pain relief in painful diabetic polyneuropathy is not dependent on GABA release in the dorsal horn of the spinal cord. CNS Neurosci Ther. 2020;26(1):136-43.

29. Du X, Hao H, Yang Y, Huang S, Wang C, Gigout S, et al. Local GABAergic signaling within sensory ganglia controls peripheral nociceptive transmission. J Clin Invest. 2017;127(5):1741-56.

30. Franken G, Debets J, Joosten EAJ. Dorsal Root Ganglion Stimulation in Experimental Painful Diabetic Peripheral Neuropathy: Burst vs. Conventional Stimulation Paradigm. Neuromodulation. 2018.

31. Franken G, Debets J, Joosten EAJ. Nonlinear Relation Between Burst Dorsal Root Ganglion Stimulation Amplitude and Behavioral Outcome in an Experimental Model of Painful Diabetic Peripheral Neuropathy. Neuromodulation. 2019.

32. Pluijms WA, van Kleef M, Honig WM, Janssen SP, Joosten EA. The effect of spinal cord stimulation frequency in experimental painful diabetic polyneuropathy. Eur J Pain. 2013;17(9):1338-46.

33. van Beek M, Hermes D, Honig WM, Linderoth B, van Kuijk SMJ, van Kleef M, et al. Long-Term Spinal Cord Stimulation Alleviates Mechanical Hypersensitivity and Increases Peripheral Cutaneous Blood Perfusion in Experimental Painful Diabetic Polyneuropathy. Neuromodulation. 2018.

34. van Beek M, van Kleef M, Linderoth B, van Kuijk SM, Honig WM, Joosten EA. Spinal cord stimulation in experimental chronic painful diabetic polyneuropathy: Delayed effect of High-frequency stimulation. Eur J Pain. 2017;21(5):795-803.

35. Chaplan SR, Bach FW, Pogrel JW, Chung JM, Yaksh TL. Quantitative assessment of tactile allodynia in the rat paw. J Neurosci Methods. 1994;53(1):55-63.

36. Mills C, Leblond D, Joshi S, Zhu C, Hsieh G, Jacobson P, et al. Estimating efficacy and drug ED50's using von Frey thresholds: impact of weber's law and log transformation. J Pain. 2012;13(6):519-23.

37. Sleigh JN, Weir GA, Schiavo G. A simple, step-by-step dissection protocol for the rapid isolation of mouse dorsal root ganglia. BMC Res Notes. 2016;9:82.

38. Koetsier E, Franken G, Debets J, van Kuijk SMJ, Perez R, Linderoth B, et al. Effectiveness of dorsal root ganglion stimulation and dorsal column spinal cord stimulation in a model of experimental painful diabetic polyneuropathy. CNS Neurosci Ther. 2018.

39. Yu G, Segel I, Zhang Z, Hogan QH, Pan B. Dorsal Root Ganglion Stimulation Alleviates Pain-related Behaviors in Rats with Nerve Injury and Osteoarthritis. Anesthesiology. 2020;133(2):408-25.

40. Meuwissen KPV, de Vries LE, Gu JW, Zhang TC, Joosten EAJ. Burst and Tonic Spinal Cord Stimulation Both Activate Spinal GABAergic Mechanisms to Attenuate Pain in a Rat Model of Chronic Neuropathic Pain. Pain Pract. 2020;20(1):75-87.

41. Graham RD, Bruns TM, Duan B, Lempka SF. Dorsal root ganglion stimulation for chronic pain modulates Abeta-fiber activity but not C-fiber activity: A computational modeling study. Clin Neurophysiol. 2019;130(6):94151.

42. Koetsier E, Franken G, Debets J, Heijmans L, van Kuijk SMJ, Linderoth B, et al. Mechanism of dorsal root ganglion stimulation for pain relief in painful diabetic polyneuropathy is not dependent on GABA release in the dorsal horn of the spinal cord. CNS Neurosci Ther. 2019.

43. Kent AR, Min X, Hogan QH, Kramer JM. Mechanisms of Dorsal Root Ganglion Stimulation in Pain Suppression: A Computational Modeling Analysis. Neuromodulation. 2018;21(3):234-46.

44. Chao D, Zhang Z, Mecca CM, Hogan QH, Pan B. Analgesic dorsal root ganglionic field stimulation blocks conduction of afferent impulse trains selectively in nociceptive sensory afferents. Pain. 2020. 
45. Lee KH, Chung K, Chung JM, Coggeshall RE. Correlation of cell body size, axon size, and signal conduction velocity for individually labelled dorsal root ganglion cells in the cat. J Comp Neurol. 1986;243(3):335-46.

46. Pawela CP, Kramer JM, Hogan QH. Dorsal root ganglion stimulation attenuates the BOLD signal response to noxious sensory input in specific brain regions: Insights into a possible mechanism for analgesia. Neuroimage. 2017;147:10-8.

47. Chapman KB, Groenen PS, Vissers KC, van Helmond N, Stanton-Hicks MD. The Pathways and Processes Underlying Spinal Transmission of Low Back Pain: Observations From Dorsal Root Ganglion Stimulation Treatment. Neuromodulation. 2020. 


\section{CHAPTER}

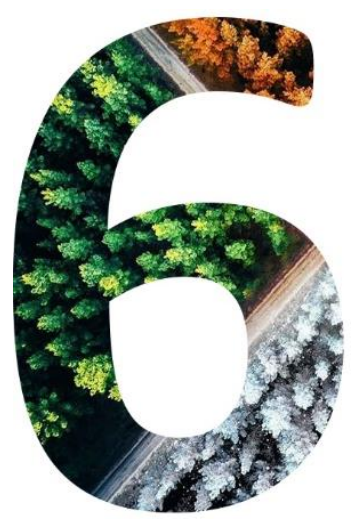

The Use of CatWalk Gait Analysis for

Assessing Chronic Neuropathic Pain in

Rats Treated with Pulsed

Radiofrequency

G. Franken, E.A. Joosten

Submitted. 


\subsection{Abstract}

Objectives: Pulsed radiofrequency (PRF) is a commonly used neuromodulation modality for treating chronic neuropathic pain. An important translational aspect of preclinical findings related to PRF is the use of new objective, accurate behavioral tests. This study aimed to 1 . study the effect of PRF on mechanical and thermal hypersensitivity and 2 . implement CatWalk gait analysis for detection of pain-related gait changes after PRF in rats with chronic neuropathic pain.

Methods: Male Sprague-Dawley rats were subjected to partial sciatic nerve ligation (PSNL) and randomly assigned to a PRF or sham group. PRF was applied to the ipsilateral L5 Dorsal Root Ganglion (DRG) two weeks post-PSNL. Animals were tested for mechanical hypersensitivity, thermal hypersensitivity and selected parameters of pain-related gait changes (CatWalk) on baseline, days 3, 7 and 14 post-PSNL, and days 5, 8 and 15 post-PRF application.

Results: PSNL successfully induced mechanical and thermal hypersensitivity throughout the 14 days post-PSNL. Additionally, significant pain-related gait changes over time were observed for all selected CatWalk parameters following PSNL injury, but changes tended to normalize 14 days after PSNL. PRF treatment attenuated PSNL-induced mechanical hypersensitivity at all post-PRF time points and thermal hypersensitivity at post-PRF days 5 and 15. No effect of PRF was observed on selected Catwalk parameters.

Conclusions: PRF treatment attenuates mechanical hypersensitivity and thermal hypersensitivity in animals with chronic neuropathic pain. Catwalk gait analysis allows for objective assessment of chronic neuropathic pain following nerve injury. CatWalk does not result in the detection of behavioral changes to PRF.

Keywords: Pulsed radiofrequency, mechanical hypersensitivity, thermal hypersensitivity, gait analysis, CatWalk, chronic neuropathic pain. 


\subsection{Introduction}

Neuropathic pain is a type of pain caused by injury or disease of the peripheral or central nervous system, affecting approximately $8 \%$ of the total adult population [1]. Since the introduction of pulsed radiofrequency (PRF) treatment by Sluijter and colleagues in 1998, PRF has consistently been used in clinical pain practice [2]. PRF relies on the administration of radiofrequency current to a peripheral nerve or dorsal root ganglion (DRG) of interest. In contrast to continuous radiofrequency (CRF) treatment, which involves ablation of nerve tissue by the process of thermocoagualation, PRF offers an isothermal alternative that keeps the temperature of the electrode tip below $42^{\circ} \mathrm{C}$ by administering radiofrequency current in a pulsed-based fashion. This circumvents the neurological side effects as seen in CRF, such as sensory loss, motor deficits, and in severe cases post-operative pain, and is thought to involve modulation rather than ablation of the nociceptive network [3-5]

Although a growing amount of literature suggests clinical efficacy of PRF for neuropathic pain on the cervical $[6]$, as well as lumbar $[2,7,8]$ level, strong clinical evidence for the use of PRF in neuropathic pain remains limited. Additionally, PRF also tends to provide positive outcomes in preclinical animal models of neuropathic pain in terms of mechanical hypersensitivity and thermal hypersensitivity, although literature shows that the effects of PRF, the used pain model, electrode positioning, time frame, and stimulation settings are subjected to great diversity between research groups [9-23]. Together, this creates the need for further validation of PRF as a treatment for neuropathic pain on both the clinical and preclinical level.

A second aspect important for validation and translationability of the preclinical findings related to effects of PRF and neuropathic pain is the use of new objective, accurate behavioral tests. To date, reflex-based outcome measures, such as mechanical hypersensitivity (e.g. Von Frey test, Randall-Selitto test) and thermal hypersensitivity (e.g. Hargreaves test, hot plate test) [24] are considered to be the golden standard in experimental pain research. More recently, CatWalk gait analysis was suggested as a novel and highly objective alternative to these reflex-based outcome measures in order to study experimental 
neuropathic pain. The Catwalk gait analysis system is a fully automated, objective, computerassisted method of locomotor analysis, allowing rapid quantification of individual paw parameters as well as parameters related to interlimb coordination [25]. CatWalk gait analysis has successfully been used as an assessment for both acute [26] and chronic neuropathic pain $[25,27]$.

It is therefore that we, for the first time, included CatWalk gait analysis to not only study the effect of partial ligation of the sciatic nerve but also the treatment effect of PRF applied adjacent to the DRG. Therefore, the aim of this study was twofold: first to analyze the behavioral effect of PRF in terms of mechanical and thermal hypersensitivity in an experimental model of chronic neuropathic pain, and second to implement CatWalk gait analysis for detection of pain-related gait changes after PRF in rats with chronic neuropathic pain.

\subsection{Methods}

\subsubsection{Experimental animals}

All experiments were performed using male Sprague-Dawley rats (6 weeks at study onset, $150-200 \mathrm{~g}, \mathrm{n}=23$ ). Animals were housed in filter-top polycarbonate cages (per 2 ) in a climate controlled room (temperature $21 \pm 1^{\circ} \mathrm{C}$, humidity $55 \pm 15 \%$ ) and under artificial lightning (12:12 reversed light/dark cycle). Food and distilled water was ad libitum available. Animals were allowed to acclimatize to the housing facility for 1 week after arriving at the animal facility, and were handled properly before starting experiments. Experiments were conducted in a humane manner and in accordance with the European Directive for the Protection of Vertebrate Animals Used for Experimental and Other Scientific Purposes (86/609/EU). The protocol was approved by the Animal Research Committee of the Maastricht University Medical Centre (DEC-protocol 2014-085).

\subsubsection{Surgeries}

All surgeries were performed under anesthesia and aseptic conditions. Anesthesia was induced using $4 \%$ Isoflurane in a Plexiglas cylinder induction chamber and maintained 
with $1-2 \%$ Isoflurane using an anesthesia mask (1:1 with air and $100 \% \mathrm{O}_{2}, 250 \mathrm{ml} / \mathrm{min}$ flow; Univentor 400 anesthesia unit, Univentor Limited, Zejtun ZTN 3000, Malta). Body temperature was controlled at $37.5^{\circ} \mathrm{C} \pm 0.5$ using an automatic heating pad and an ointment was applied to the eyes prior to each surgery in order to prevent eye dehydration. Surgeries were started in case of absence of a toe and eye-ball reflex.

\subsubsection{Partial sciatic nerve ligation (PSNL)}

A unilateral ligation of the left sciatic nerve was induced $(n=23)$ as described by Seltzer et al. [28], and as previously applied in our laboratory [29, 30]. Briefly, the skin covering the left hind paw was shaved using an electrical shaving apparatus and was disinfected with povidine-iodine. The animal was placed face down on a heating pad and a small incision was made at high thigh level using a carbon steel surgical blade no. 10 . The left sciatic nerve was exposed by blunt dissection, and the dorsum of the nerve was carefully freed from surrounding connective tissue at a site near the trochanter just distal to the Nervus biceps semitendinosus posterior under a magnification of 25 times. An 8-0 nonabsorbable silk suture was inserted into the sciatic nerve using a reverse cutting micro needle in a way that one-third to one-half of the diameter of the sciatic nerve was trapped inside the ligature. Lastly, the surgical wound was intracutaneously closed using 4-0 silk sutures and the animal was allowed to recover for at least two days.

\subsubsection{Pulsed radiofrequency (PRF) procedure}

Two weeks after PSNL surgery, animals were randomly assigned to either a PRF $(n=12)$ or sham-PRF group $(n=9)$ using the website randomize.org. The back and torso of the animal were shaved using an electrical shaving apparatus and disinfected with povidineiodine. The animal was placed face down on a disposable radiofrequency grounding pad (DGP-PM, Cosman Medical Inc., Burlington, MA, USA) treated with ultrasound transmission gel (Aquasonic 100, Parker Laboratories Inc., Fairfield, CT, USA). The spinous process of L6 was identified at the level of the pelvic bone and a skin incision covering spinous process $L 4$ to L6 was made using a carbon steel surgical blade no. 10. The left L5 DRG was then exposed by laminectomy and facetectomy. PRF waves were applied using a PRF generator (Cosman 
G4 generator, Cosman Medical Inc., Burlington, MA, USA), by placing an electrode $(10 \mathrm{~cm}, 5$ mm tip, 22 Gauge) adjacent to the left L5 DRG. PRF stimulation parameters were set as follows: pulse frequency $=2 \mathrm{~Hz}$, pulse width $=20 \mathrm{~ms}$, output voltage $=45 \mathrm{~V}$, stimulation duration $=2 \times 180 \mathrm{~s}$ and maximum temperature $=42{ }^{\circ} \mathrm{C}$ (kept $<42^{\circ} \mathrm{C}$ by varying pulse width). Animals in the sham-PRF group underwent the exact same surgery procedure as the PRF group, including placement of the electrode adjacent to the L5 DRG. However, the PRF generator was turned off to not allow stimulation of the DRG. Lastly, the surgical wound was closed using 4-0 silk sutures and the animal was allowed to recover for at least four days.

\subsubsection{Behavioral analysis}

At baseline ( $t=0), t=3,7$, and 14 days following $P S N L$, and $t=5,8,15$ days following PRF treatment, nociceptive behavior was assessed by measuring mechanical hypersensitivity (Von Frey), thermal hypersensitivity (Hargreaves), and pain-related gait changes (CatWalk) (Figure 1). An interval of at least two hours was used between two consecutive measurements. The experimenter was blinded to the experimental condition of the animals.

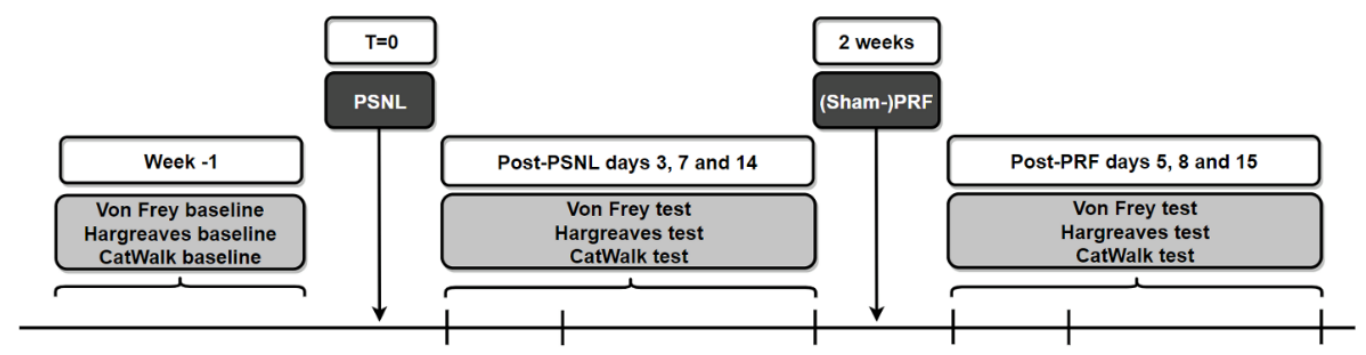

Figure 1. Study design.

\subsubsection{Mechanical hypersensitivity (Von Frey assay)}

Mechanical hypersensitivity was assessed by measuring paw withdrawal thresholds (PWT) of the hind paws to mechanical stimulation with von Frey filaments. Animals were individually placed in an in-house developed closed Plexiglas cage chamber set-up with metal mesh floor in a dark room with ambient lighting. Animals were placed in the set-up for 15 minutes before each test in order to acclimatize the animals to the testing environment. $A$ 
series of von Frey filaments with logarithmic incremental stiffness (Stoelting, Wood Dale, IL, USA; bending forces $0.6,1.2,2.0,3.6,5.5,8.5,15.1$ and $28.84 \mathrm{~g}$ ) was applied to the plantar surface of the hind paws of the rats for 5 seconds, using the up-down method as described by Chaplan et al. [31]. If the hind paw was not withdrawn, the next filament with greater bending force was applied, whereas the previous filament with lower bending force was applied if the hind paw was withdrawn. A positive response was defined as a swift, sharp paw withdrawal response upon application of the filament to the hind paw. The 50\% PWT was calculated after completion of a sequence of six consecutive responses [32]. The $28.84 \mathrm{~g}$ filament was taken as cut-off value to prevent tissue damage, and, if no withdrawal response was elicited at $28.84 \mathrm{~g}$, the mechanical paw withdrawal threshold was noted as $28.84 \mathrm{~g}$. Finally, 50\% PWTs were multiplied by 10.000 and logarithmically transformed to obtain a linear scale and to account for Weber's law [33]

\subsubsection{Thermal hypersensitivity (Hargreaves assay)}

Thermal hypersensitivity was tested by measuring the paw withdrawal latency (PWL) of the hind paws to thermal stimulation using a Hargreaves apparatus (Plantar Test apparatus 37370, Ugo Basile, Milan, Italy). Animals were individually placed on the glass floor of the Hargreaves apparatus in transparent plastic cages in a room with ambient lighting. Animals were placed in the set-up for 15 minutes before each test in order to acclimatize them to the testing surroundings. A focused radiant heat source underneath the glass floor with increasing temperature was then applied to the plantar surface of the hind paw. A swift, sharp withdrawal of the hind paw was considered a positive response and measurements were only taken when the rat was not moving. The PWL of both hind paws of each rat was measured four times, and the mean value of the last three measurements was used for analysis. A cut-off value of 20 s was used to avoid tissue injury.

\subsubsection{Gait analysis (CatWalk)}

The gait of the animals was analyzed using the CatWalk quantitative gait analysis system (Version 9.1, Noldus, Wageningen, The Netherlands). For a complete description of the CatWalk system, the reader is referred to the paper by Hamers et al. [34]. In brief, the 
CatWalk consists of a glass runway with plastic walls that is capable of specifically illuminating an animal's footprints. Animals are filmed from underneath the glass runway in a darkened room, thus providing good contrast between paw prints and the rest of the body. The CatWalk behavioral analysis system is a computer-assisted method of locomotor analysis that allows a rapid quantification of individual paw parameters as well as parameters related to interlimb coordination. Two weeks prior to the start of the experiment, the animals are trained to cross the runway and acclimatized to the testing procedure. In order to motivate the animals to cross the runway, animals are rewarded with small sucrose pellets after each run. For correct gait analysis, the following criteria for correct runway crossing have to be met: (1) the rat crossed the runway without interruption or hesitation within five s and (2) a minimum of three correct runs per animal have to be required. The following CatWalk parameters were analyzed in the present study: (1) stand phase: the duration in seconds of contact of a paw with the glass plate, (2) print area: the surface area of the complete paw print, (3) mean intensity: the mean intensity of the paw print, (4) max intensity: the maximum intensity of the paw print.

\subsubsection{Statistical analysis}

Data were tested for normality using the D'Agostino \& Pearson omnibus normality test. Von Frey data were multiplied by 10.000 and logarithmically transformed to obtain a linear scale and to account for Weber's law [33]. For the statistical analysis of differences in mechanical PWTs over time, one-way repeated measures analysis of variance (ANOVA) was used, followed by Dunnett's post hoc test. For the comparison of PWTs between groups and between the ipsi- and contralateral hind paw for each individual time point, independent samples T-test and paired samples T-test were used, respectively. Changes in thermal PWLS and CatWalk gait parameters over time were analyzed using the non-parametric Friedman test followed by Dunn's post hoc test. Differences in thermal PWLs and CatWalk gait parameters between groups and between the ipsi- and contralateral hind paw for each individual time point were analyzed using the nonparametric Mann-Whitney-U-test and Wilcoxon matched-pairs signed rank test, respectively. Data are presented as mean \pm 
standard error of the mean (SEM). P-values $<0.05$ were considered to indicate statistical significance. All statistical analyses were performed using Graphpad Prism 6.

\subsection{Results}

\subsubsection{Development of chronic neuropathic pain}

\subsubsection{Mechanical hypersensitivity (Von Frey assay)}

A significant decrease in terms of $\log _{10}(10.000 \times 50 \%$ PWT) responses over time was observed in the ipsilateral hind paw when compared to baseline $(p<0.0001)$. The average $\log _{10}(10.000 \times 50 \%$ PWT) of the ipsilateral hind paw was $5.0 \pm 0.3$ at baseline. After PSNL, $\log _{10}(10.000 \times 50 \%$ PWT) responses significantly decreased to $4.1 \pm 0.2$ at PO-3d $(p<0.0001)$ $4.1 \pm 0.2$ at PO-7d $(p<0.0001)$ and $4.0 \pm 0.3$ at PO-14d $(p<0.0001)$. Interestingly, a significant reduction in terms of $\log _{10}(10.000 \times 50 \%$ PWT) responses was also observed in the contralateral hind paw $(\mathrm{p}<0.01)$, albeit at reduced magnitude. The average $\log _{10}(10.000 \mathrm{x}$ $50 \%$ PWT) of the contralateral hind paw was $5.0 \pm 0.3$ at baseline. After PSNL, $\log _{10}(10.000$ $x 50 \%$ PWT) responses decreased to $4.9 \pm 0.3$ at PO-3d $(p=0.21), 4.8 \pm 0.3$ at PO-7d $(p<0.01)$ and $4.9 \pm 0.3$ at PO-14d $(\mathrm{p}<0.05)$. Importantly, statistical significant differences were also

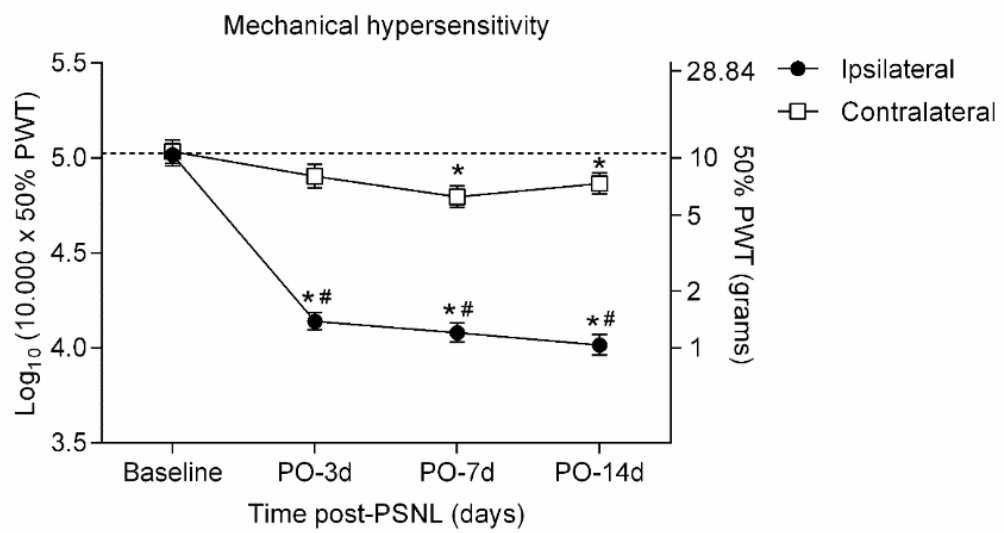

Figure 2. Development of PSNL-induced mechanical hypersensitivity. ${ }^{*} p<0.0001$ compared to baseline. $\#$ p $<0.0001$ compared to the contralateral hind paw at the same time point. Data are presented at as mean \pm SEM. $\mathrm{PO}=$ postoperative day. Dotted line= average baseline before PSNL. 
found between the ipsi- and contralateral paw at PO-3d (p<0.0001), PO-7d 3d $(p<0.0001)$ and PO-14d ( $p<0.0001)$ (Figure 2).

\subsubsection{Thermal hypersensitivity (Hargreaves assay)}

A significant decrease in terms of thermal PWLs over time was observed in the ipsilateral hind paw when compared to baseline $(p<0.0001)$. The average thermal PWL of the ipsilateral hind paw was $8.9 \pm 3.0 \mathrm{~s}$ at baseline. After PSNL, thermal PWLs decreased to $5.5 \pm$ $1.4 s$ at $P O-3 d(p<0.0001), 5.5 \pm 1.6 s$ at PO-7d $(p<0.0001)$ and $5.9 \pm 2.0$ s at PO- $14 d(p<0.001)$. No significant reductions in terms of thermal PWLs were observed in the contralateral hind paw $(p=0.11)$. The average thermal PWL of the contralateral hind paw was $8.9 \pm 3.0 \mathrm{~s}$ at baseline. After PSNL, thermal PWLs remained stable at $9.0 \pm 2.7 \mathrm{~s}$ at PO-3d ( $>>0.99), 8.3 \pm 2.1$ $s$ at PO-7d $(p=0.63)$ and $9.7 \pm 2.2 s$ at PO-14d ( $=0.76)$. Importantly, statistical significant differences were also found between the ipsi- and contralateral paw at PO-3d $(p<0.0001)$, PO-7d $(p<0.0001)$ and PO-14d ( $p<0.0001)$ (Figure 3).

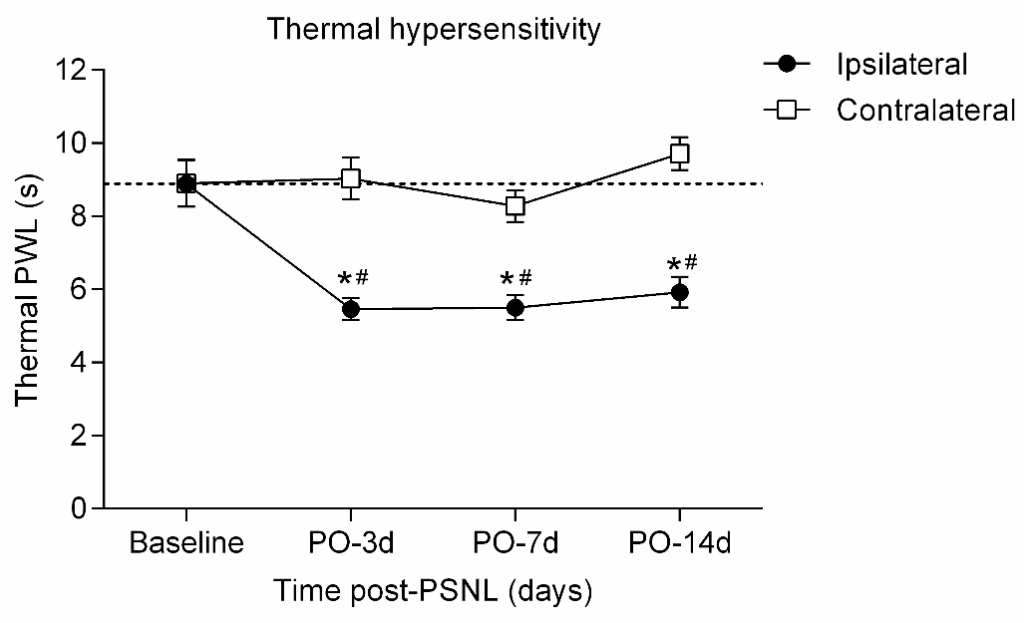

Figure 3. Development of PSNL-induced thermal hypersensitivity. ${ }^{*} p<0.001$ compared to baseline. $\# p<0.0001$ compared to the contralateral hind paw at the same time point. Data are presented at as mean $\pm \mathrm{SEM}$. $\mathrm{PO}=$ postoperative day. Dotted line= average baseline before PSNL. 


\subsubsection{Gait analysis (CatWalk)}

Significant decreases over time were observed in the ipsilateral hind paw for all selected CatWalk parameters. A significant decrease in stand phase over time was observed in the ipsilateral hind paw when compared to baseline $(p<0.05)$, with a reduction in stand phase of $18 \%, 20 \%$, and $14 \%$ at days $3(p<0.05), 7(p<0.05)$, and $14(p=0.31)$ following PSNL, respectively. Additionally, a significant decrease in print area over time was observed in the ipsilateral hind paw when compared to baseline $(p<0.05)$, with a reduction in print area of $24 \%, 16 \%$ and $8 \%$ at days $3(p=0.06), 7(p=0.19)$ and 14 ( $p>0.99)$ following PSNL. Also mean intensity was significantly changed over time $(p<0.05)$ : $9 \%$ at 3 PO-3d $(p>0.99), 6 \%$ at PO-7d $(p>0.99)$ and $4 \%$ at PO-14d ( $p=0.06)$. Lastly, also max intensity of the ipsilateral hind paw was significantly decreased over time $(p<0.01)$. Max intensity of the ipsilateral hind paw decreased with $17 \%, 11 \%$, and $2 \%$ at days $(p<0.05), 7(p=0.11)$ and $14(p>0.99)$ following PSNL (Figure 4).

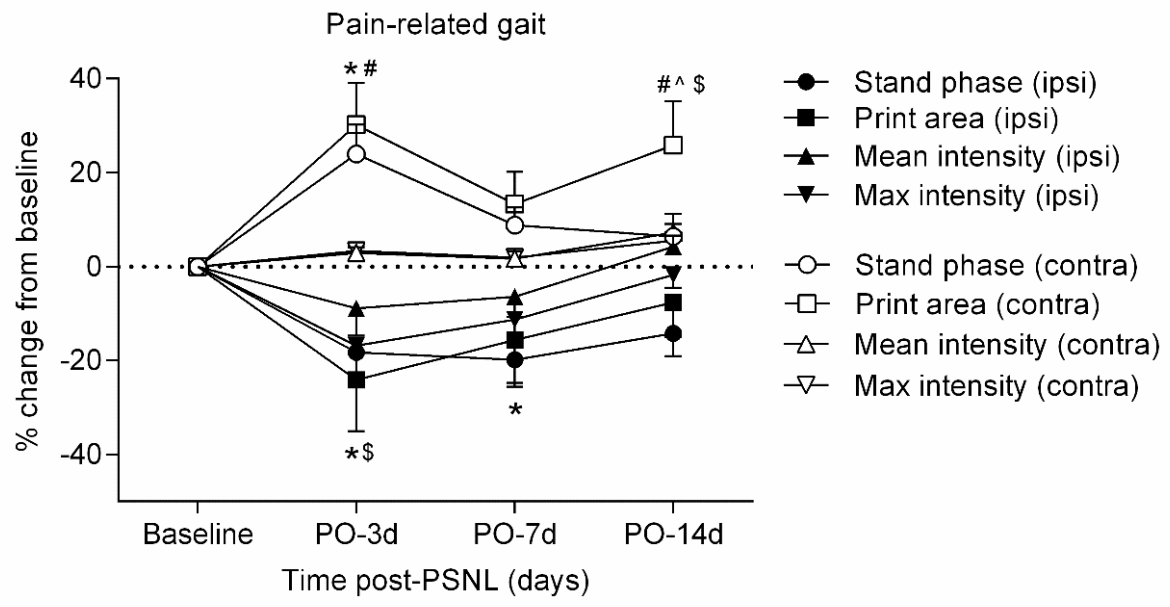

Figure 4. Development of pain-related gait changes. ${ }^{*} \mathrm{p}<0.05$ stand phase compared to baseline; $\# p<0.05$ print area compared to baseline; ${ }^{\wedge} p<0.01$ mean intensity compared to baseline; $\$ p<0.05$ max intensity compared to baseline. Data are presented at as mean \pm SEM and presented as percentage change from (pre-PSNL) baseline. $\mathrm{PO}=$ postoperative day.

Interestingly, significant increases over time were observed in the contralateral paw for all selected CatWalk parameters: stand phase; $p<0.05$, print area; $p<0.01$, mean intensity; 
$p<0.01$, max intensity; $p<0.01$. Stand phase increased with $24 \%, 9 \%$ and $6 \%$ at days 3 $(p<0.05), 7(p=0.60)$ and $14(p>0.99)$ compared to baseline. Print area increased with $30 \%$, $13 \%$ and $26 \%$ at days $3(p<0.01), 7(p=0.60)$ and $14(p<0.05)$ compared to baseline. Mean intensity increased with $3 \%, 2 \%$ and $7 \%$ at days $3(p=0.11), 7(p=0.48)$ and $14(p<0.01)$ compared to baseline. Max intensity increased with $3 \%, 2 \%$ and $7 \%$ at days 3 ( $p=0.19), 7$ $(p=0.60)$ and $14(p<0.001)$ compared to baseline (Figure 4).

Except for print area at post-PSNL day 7, and mean intensity at post-PSNL day 14 , a significant difference was observed between the ipsi- and contralateral hind paw for each CatWalk parameter at each time point $(p<0.05)$ (Figure 4).

\subsubsection{Effect of pulsed radiofrequency treatment}

\subsubsection{Mechanical hypersensitivity (Von Frey)}

PRF adjacent to the L5 DRG significantly attenuated PSNL-induced mechanical hypersensitivity in the ipsilateral hind paw over time $(p<0.0001)$. $\log _{10}(10.000 \times 50 \%$ PWT) values increased from $4.1 \pm 0.2$ at PO-14d to $4.6 \pm 0.3$ at PRF-5d ( $p<0.001), 4.7 \pm 0.2$ at PRF$8 d(p<0.0001)$ and $4.6 \pm 0.3$ at PRF-15d $(p<0.001)$. Sham-PRF had no effect on $\log _{10}(10.000 x$

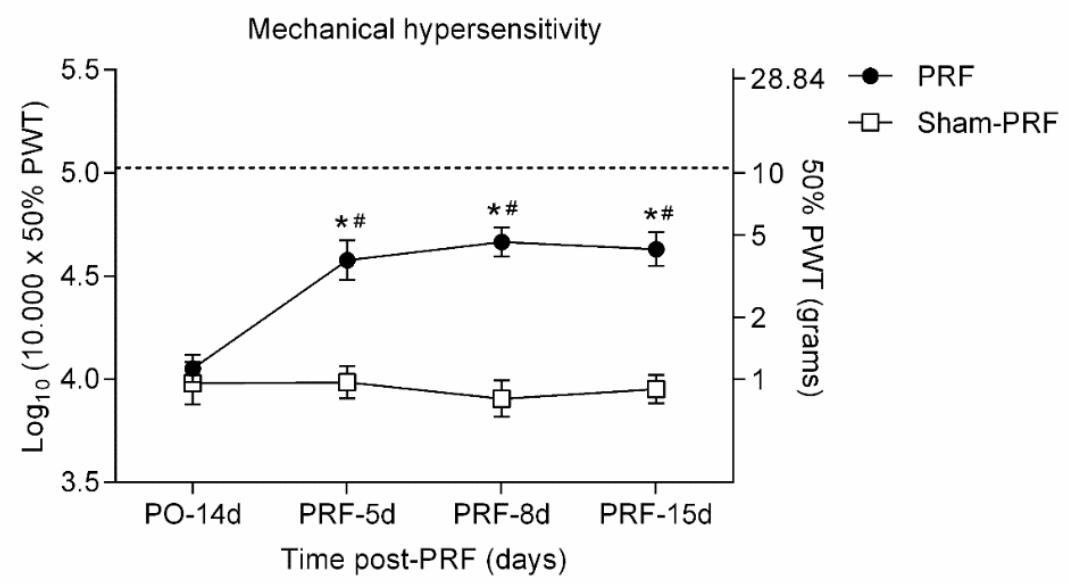

Figure 5. Effect of PRF treatment adjacent to the L5 DRG on PSNL-induced mechanical hypersensitivity. ${ }^{*} p<0.001$ compared to PO-14d. \#p<0.001 compared to the sham-PRF group at the same time point. Data are presented at as mean \pm SEM. POd=postoperative day. Dotted line= average baseline before PSNL. 
$50 \%$ PWT) values of the ipsilateral paw over time $(p=0.71)$. Additionally, there was a significant intergroup difference between the PRF and sham-PRF group in terms of $\log _{10}$ $(10.000 \times 50 \%$ PWT) at all time points following PRF treatment $(p<0.001)$ (Figure 5).

\subsubsection{Thermal hypersensitivity (Hargreaves)}

PRF adjacent to the L5 DRG also significantly attenuated PSNL-induced thermal hypersensitivity in the ipsilateral hind paw over time $(p<0.01)$. PWLs increased from $5.6 \pm 0.5$ $s$ at PO-14d to $7.7 \pm 0.4 s$ at PRF-5d $(p<0.05), 7.7 \pm 0.3 s$ at PRF-8d $(p=0.05)$ and $9.4 \pm 1.0 s$ at PRF-15d $(p<0.01)$. Sham-PRF showed no effect on PWLs of the ipsilateral paw over time $(p=0.35)$. Lastly, significant differences were found between PWLs of the PRF and sham-PRF group at all time points following PRF treatment $(p<0.05)$ (Figure 6).

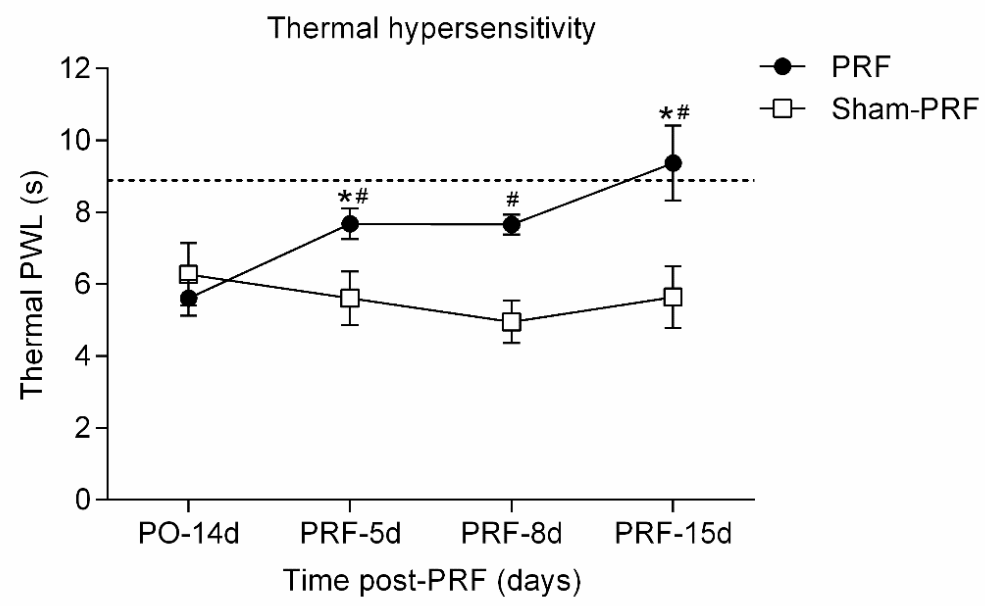

Figure 6. Effect of PRF treatment adjacent to the L5 DRG on PSNL-induced thermal hypersensitivity. ${ }^{*} p<0.05$ compared to PO-14d. \#p<0.05 compared to the sham-PRF group at the same time point. Data are presented at as mean \pm SEM. POd=postoperative day. Dotted line= average baseline before PSNL.

\subsubsection{Gait analysis (CatWalk)}

With use of CatWalk, no significant effect of PRF was observed for any of the tested gait parameters. Additionally, no effects were observed for any of the selected gait parameters following Sham-PRF (Figure 7). 


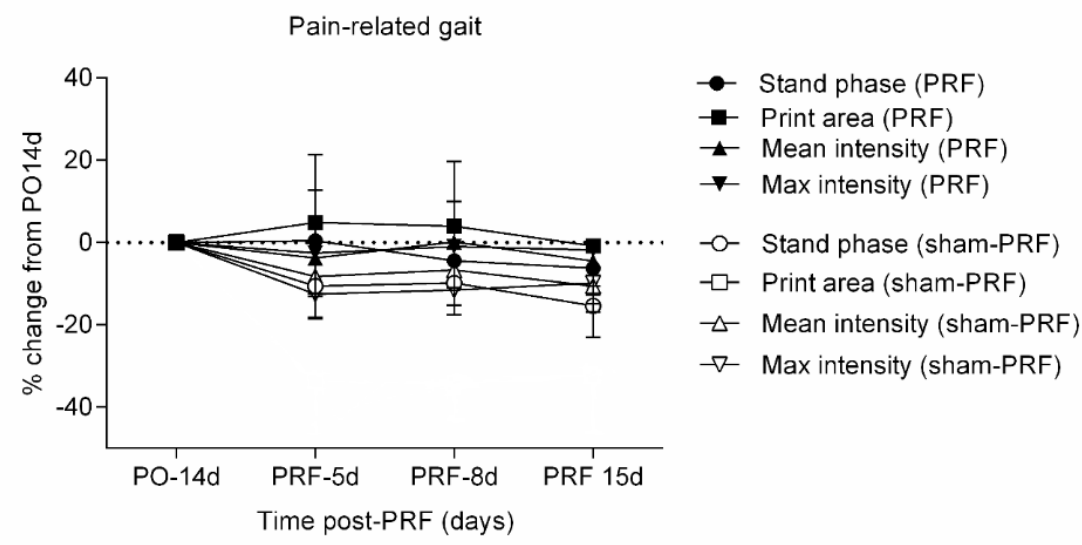

Figure 7. Effect of PRF treatment adjacent to the L5 DRG on pain related gait changes. Data are presented at as mean \pm SEM and presented as percentage change from (pre-PSNL) baseline. $P O=$ postoperative day.

\subsection{Discussion}

Our study showed that a single treatment with PRF adjacent to the ipsilateral L5DRG significantly attenuates PSNL-induced mechanical hypersensitivity as well as thermal hypersensitivity in an animal model of chronic neuropathic pain. With this we confirm and validate previous findings (9-23), which is an essential part of establishing a reproducible and valid animal model for studying the effects of PRF in the treatment of chronic neuropathic pain. PSNL, first described by Seltzer et al. in 1998 [28], resulted in the development of chronic neuropathic pain, which is in line with previous studies using this same model [28, 35-38]. PSNL is often considered the golden standard for modeling clinical chronic neuropathic pain in rodents, because of the immediate onset (within a few hours) and longlasting perpetuation (at least 7 months) of translational symptoms like mechanical hypersensitivity and thermal hypersensitivity. Additionally, animals that undergo PSNL show signs of spontaneous pain, like paw guarding and licking, but do not show signs of rather extreme spontaneous pain behavior, like autotomy, as observed in other widely adopted preclinical pain models including the Chronic Constriction Injury $(\mathrm{CCI})[28,39]$. The effect of PRF on mechanical as well as thermal hypersensitivity has been demonstrated in various experimental studies and a wide variety of neuropathic pain models, including PSNL [13], CCI 
$[14,22]$, Spinal Nerve Ligation (SNL) $[10,11,16,21]$, Spared Nerve Injury $[15,19,23]$, Nucleus Pulposus administration to the dorsal root [17], Complete Freund's Adjuvant (CFA) injection into the hind paws [12, 18, 20], and intraperitoneal resiniferatoxin injection [9]. However, comparing the outcomes of these studies remains difficult, as not only the used model differs between studies, but studies are also subject to great diversity in terms of the used PRF location and PRF duration. Interestingly, studies by Chen et al.[18] and Yang et al. [20] found that PRF adjacent the DRG, but not PRF adjacent to the sciatic nerve, reduced mechanical hypersensitivity in a CFA-induced inflammatory pain model $[18,20]$, favoring the use of DRGPRF over sciatic nerve PRF. Additionally, no consensus has been reached with respect to the optimal duration of PRF. While some studies favor 2 minutes PRF over 6 minutes PRF [11], other studies favor 6 minutes over 2 minutes PRF [9]. Our PRF protocol of 2x 180 s PRF applied adjacent to the L5 DRG showed a profound attenuation of PWT and PWL values for up to 15 days. Interestingly, a study by Perret et al. showed that animals that received PRF treatment adjacent to the L5 DRG exhibited better recovery and showed larger effect sizes than sham treated animals for a period up to no less than 50 days post-PRF treatment [10]. Indeed, both our Von Frey and Hargreaves data show no sign of attenuation in terms of PRF treatment efficacy at post-PRF day 15 , making it likely that the pain relieving effect of PRF would have persisted after 15 days.

Besides reflex-based tests like the Von Frey and Hargreaves assay, several attempts have been undertaken to measure behavioral changes in animal models of pain, as an indirect measure of pain. Over the years, CatWalk gait analysis has been demonstrated to be one the most complete and objective methods of measuring gait in experimental animal research $[25,34,40]$. The Catwalk gait analysis system is a computer-assisted method of locomotor analysis, allowing rapid quantification of individual paw parameters as well as parameters related to interlimb coordination [25]. The CatWalk system has been used in experimental animal research for a wide variety of neurological disorders, ranging from spinal cord injury $[41,42]$ and peripheral nerve injury $[25,27,43]$ to traumatic brain injury [44] and Parkinson's disease [45]. The addition of the CatWalk gait analysis to our set of behavioral outcome assessments was decided upon several reasons: 1 . To provide an 
objective and quantitative alternative for measuring neuropathic pain in addition to reflexbased assessments like the Von Frey and Hargreaves test. 2. To provide analysis of more subtle pain related gait changes which then might allow analysis of the PRF effect in more detail. In our study, CatWalk gait analysis of animals that underwent PSNL showed significant decreases over time for all selected CatWalk parameters. Moreover, a significant ipsilateralcontralateral difference was observed for all Catwalk parameters at almost each time point. This observation, in combination with the fact that significant increases were found for all selected parameters in the contralateral hind paw, strongly suggest a compensatory role of the contralateral hind paw, by unloading the pain at the ipsilateral hind paw. In comparison, a study by Vrinten et al. reported significant decreases in both stand phase and mean intensity of the ipsilateral hind-paw following chronic constriction injury (CCl) for at least 5 weeks [27]. These changes in stand phase and mean intensity of paw print normalized at 10 weeks. Although the time frame of normalization in our study was substantially different compared to the study of Vrinten et al., our results also showed a gradual normalization of Catwalk parameters over time, possibly hinting to development of adaptation behavior in the animals. Using the same $\mathrm{CCl}$ model, another study showed stand phase, print area, and max intensity to be significantly decreased following ligation for a period up to 28 days [46]. Furthermore, Gabriel and colleagues showed that intensity and print area of the ipsilateral hind paw significantly decreased using an acute inflammatory pain model of carrageenan injection into the knee for a period up to 48 hours [26]. With regard to this last study, the same authors also found significant decrease in intensity of the ipsilateral hind paw for at least 21 days using a more chronic version of this pain model [25]. Interestingly, Truin et al., who also used the PSNL model for studying chronic neuropathic pain, albeit in the mouse, reported significant decreases in mean intensity of the ipsilateral hind paw for 14 days postsurgery, and significant decreases in stand phase until day 7 post-surgery [47]. The latter is comparable with the results for stand phase as noted in our study. However, it is worth noticing that the study by Truin et al. was performed in mice instead of rats, and that strain differences in one species (rats) were already sufficient to show marked differences in static paw parameters on the CatWalk [48]. 
Clearly, we were not able to detect any effect of PRF on CatWalk pain induced gait parameters. In contrast, and as mentioned earlier, a profound effect of PRF treatment was observed on PWTs on the Von Frey and PWLs on the Hargreaves test. In line with this, the study by Truin and colleagues, using the same animal model, showed no effect of spinal cord stimulation on any of the measured CatWalk gait parameters, but did show profound increases in PWTs on the Von Frey test [47]. Although spinal cord stimulation and pulsed radiofrequency treatment are fundamentally different in their properties and proposed working mechanisms, the fact that two of the most widely adopted interventional pain treatment approaches seem to not alter pain-induced gait, while at the same time attenuating mechanical hypersensitivity and thermal hypersensitivity in chronic neuropathic animals, strongly suggests that CatWalk gait analysis does not allow to detect or analyze behavioral effects of interventional treatment approaches in the chronic experimental PSNL model. One could speculate that the window for treatment effect of PRF on the CatWalk in this study is too small, and that the CatWalk is not sensitive enough to detect potential minor differences in terms of pain-induced gait. As this might be the case, future developments and improvements in the CatWalk system, allowing even more precise assessment of painrelated gait parameters, might allow assessment of behavioral effects due to interventional procedures.

The fact that a single treatment with PRF adjacent to the DRG has a long-lasting effect (15 days in the present study) on mechanical hypersensitivity and thermal hypersensitivity, needs a neurobiological and mechanistic understanding of PRF-induced changes in the nociceptive network. Mechanistically, PRF is thought to involve neuromodulation rather than neuro-ablation of the nociceptive network [3, 5]. Van Zundert et al. provided a first insight into the mechanism underlying PRF treatment [49]. The authors observed an increase in c-Fos immunoreactive cells, an indirect marker for neuronal activity, in the dorsal horn 7 days after PRF administration adjacent to the DRG. Over the years, many working mechanisms of PRF have been proposed, including modulation of excitatory neurotransmitter release in the dorsal horn [20], activation of glial cells [16, 17], activation of descending inhibitory pathways [12], and the induction of ultrastructural changes in 
important organelles of sensory c-fibers [50-52]. Additionally, a recent study found that lowvoltage bipolar PRF alleviates long-lasting neuropathic pain by selectively and persistently modulating C-fiber-mediated spinal nociceptive hypersensitivity and long-term depression [53].

In line with this, the PSNL-model, and our observation that a single PRF-treatment adjacent to the DRG attenuates mechanical as well as thermal hypersensitivity for at last two weeks, allows us to further analyze the underlying neuromodulatory mechanism of action of PRF in the near future.

\subsection{Conclusion}

We have shown that PSNL significantly induces chronic neuropathic pain as based on the presence of mechanical hypersensitivity, thermal hypersensitivity and pain-related gait changes on the CatWalk. PRF adjacent to the L5 DRG results in attenuation of chronic neuropathic pain as it attenuates mechanical and thermal hypersensitivity. Use of CatWalk based gait analysis did not allow the detection of PRF induced changes on selected CatWalk parameters. Future research should focus on elucidating the mechanisms underlying PRF treatment and optimization of the stimulation paradigm but also on further improving the CatWalk system to detect smaller changes in gait parameters. 


\subsection{References}

1. International Association for the Study of Pain. Retrieved 9 June 2016.

2. Sluijter ME CE, Rittman W, van Kleef M. The effect of pulsed radiofrequency fields applied to the dorsal root ganglion: A preliminary report. Pain Clin. 1998(11):109-17.

3. Chua NH, Vissers KC, Sluijter ME. Pulsed radiofrequency treatment in interventional pain management: mechanisms and potential indications-a review. Acta Neurochir (Wien). 2011;153(4):763-71.

4. Cosman ER, Jr., Cosman ER, Sr. Electric and thermal field effects in tissue around radiofrequency electrodes. Pain Med. 2005;6(6):405-24.

5. Sluijter ME, Imani F. Evolution and mode of action of pulsed radiofrequency. Anesth Pain Med. 2013;2(4):139-41.

6. Van Zundert J, Patijn J, Kessels A, Lame I, van Suijlekom H, van Kleef M. Pulsed radiofrequency adjacent to the cervical dorsal root ganglion in chronic cervical radicular pain: a double blind sham controlled randomized clinical trial. Pain. 2007;127(1-2):173-82.

7. Teixeira A, Grandinson M, Sluijter ME. Pulsed radiofrequency for radicular pain due to a herniated intervertebral disc--an initial report. Pain Pract. 2005;5(2):111-5.

8. Van Boxem K, de Meij N, Kessels A, Van Kleef M, Van Zundert J. Pulsed radiofrequency for chronic intractable lumbosacral radicular pain: a six-month cohort study. Pain Med. 2015;16(6):1155-62.

9. Tanaka N, Yamaga M, Tateyama S, Uno T, Tsuneyoshi I, Takasaki M. The effect of pulsed radiofrequency current on mechanical allodynia induced with resiniferatoxin in rats. Anesth Analg. 2010;111(3):784-90.

10. Perret DM, Kim DS, Li KW, Sinavsky K, Newcomb RL, Miller JM, et al. Application of pulsed radiofrequency currents to rat dorsal root ganglia modulates nerve injury-induced tactile allodynia. Anesth Analg. 2011;113(3):6106.

11. Ozsoylar O, Akcali D, Cizmeci P, Babacan A, Cahana A, Bolay H. Percutaneous pulsed radiofrequency reduces mechanical allodynia in a neuropathic pain model. Anesth Analg. 2008;107(4):1406-11.

12. Hagiwara S, Iwasaka H, Takeshima N, Noguchi T. Mechanisms of analgesic action of pulsed radiofrequency on adjuvant-induced pain in the rat: roles of descending adrenergic and serotonergic systems. Eur $\mathrm{J}$ Pain. 2009;13(3):249-52.

13. Aksu R, Ugur F, Bicer C, Menku A, Guler G, Madenoglu H, et al. The efficiency of pulsed radiofrequency application on L5 and 16 dorsal roots in rabbits developing neuropathic pain. Reg Anesth Pain Med. 2010;35(1):11 5.

14. Laboureyras E, Rivat C, Cahana A, Richebe P. Pulsed radiofrequency enhances morphine analgesia in neuropathic rats. Neuroreport. 2012;23(9):535-9.

15. Wu B, Ni J, Zhang C, Fu P, Yue J, Yang L. Changes in spinal cord met-enkephalin levels and mechanical threshold values of pain after pulsed radio frequency in a spared nerve injury rat model. Neurol Res. 2012;34(4):40814.

16. Park HW, Ahn SH, Son JY, Kim SJ, Hwang SJ, Cho YW, et al. Pulsed radiofrequency application reduced mechanical hypersensitivity and microglial expression in neuropathic pain model. Pain Med. 2012;13(9):1227-34.

17. Cho HK, Cho YW, Kim EH, Sluijter ME, Hwang SJ, Ahn SH. Changes in pain behavior and glial activation in the spinal dorsal horn after pulsed radiofrequency current administration to the dorsal root ganglion in a rat model of lumbar disc herniation: laboratory investigation. J Neurosurg Spine. 2013;19(2):256-63.

18. Chen KH, Yang CH, Juang SE, Huang HW, Cheng JK, Sheen-Chen SM, et al. Pulsed radiofrequency reduced complete Freund's adjuvant-induced mechanical hyperalgesia via the spinal c-Jun N-terminal kinase pathway. Cell Mol Neurobiol. 2014;34(2):195-203.

19. Vallejo R, Tilley DM, Williams J, Labak S, Aliaga L, Benyamin RM. Pulsed radiofrequency modulates pain regulatory gene expression along the nociceptive pathway. Pain Physician. 2013;16(5):E601-13.

20. Yang $\mathrm{CH}$, Chen $\mathrm{KH}$, Huang $\mathrm{HW}$, Sheen-Chen SM, Lin CR. Pulsed radiofrequency treatment attenuates increases in spinal excitatory amino acid release in rats with adjuvant-induced mechanical allodynia. Neuroreport. 2013;24(8):431-6.

21. Lin ML, Lin WT, Huang RY, Chen TC, Huang SH, Chang CH, et al. Pulsed radiofrequency inhibited activation of spinal mitogen-activated protein kinases and ameliorated early neuropathic pain in rats. Eur J Pain. 2014;18(5):659-70.

22. Li DY, Meng L, Ji N, Luo F. Effect of pulsed radiofrequency on rat sciatic nerve chronic constriction injury: a preliminary study. Chin Med J (Engl). 2015;128(4):540-4. 
23. Yeh CC, Wu ZF, Chen JC, Wong CS, Huang CJ, Wang JS, et al. Association between extracellular signalregulated kinase expression and the anti-allodynic effect in rats with spared nerve injury by applying immediate pulsed radiofrequency. BMC Anesthesiol. 2015;15:92.

24. Deuis JR, Dvorakova LS, Vetter I. Methods Used to Evaluate Pain Behaviors in Rodents. Front Mol Neurosci. 2017;10:284.

25. Gabriel AF, Marcus MA, Walenkamp GH, Joosten EA. The CatWalk method: assessment of mechanical allodynia in experimental chronic pain. Behav Brain Res. 2009;198(2):477-80.

26. Gabriel AF, Marcus MA, Honig WM, Walenkamp GH, Joosten EA. The CatWalk method: a detailed analysis of behavioral changes after acute inflammatory pain in the rat. J Neurosci Methods. 2007;163(1):9-16.

27. Vrinten DH, Hamers FF. 'CatWalk' automated quantitative gait analysis as a novel method to assess mechanical allodynia in the rat; a comparison with von Frey testing. Pain. 2003;102(1-2):203-9.

28. Seltzer Z, Dubner R, Shir Y. A novel behavioral model of neuropathic pain disorders produced in rats by partial sciatic nerve injury. Pain. 1990;43(2):205-18.

29. Truin M, Janssen SP, van Kleef M, Joosten EA. Successful pain relief in non-responders to spinal cord stimulation: the combined use of ketamine and spinal cord stimulation. Eur J Pain. 2011;15(10):1049 e1-9.

30. Truin M, van Kleef M, Linderoth B, Smits H, Janssen SP, Joosten EA. Increased efficacy of early spinal cord stimulation in an animal model of neuropathic pain. Eur J Pain. 2011;15(2):111-7.

31. Chaplan SR, Bach FW, Pogrel JW, Chung JM, Yaksh TL. Quantitative assessment of tactile allodynia in the rat paw. Journal of neuroscience methods. 1994;53(1):55-63.

32. Dixon WJ. Efficient analysis of experimental observations. Annu Rev Pharmacol Toxicol. 1980;20:441-62.

33. Mills C, Leblond D, Joshi S, Zhu C, Hsieh G, Jacobson P, et al. Estimating efficacy and drug ED50's using von Frey thresholds: impact of weber's law and log transformation. J Pain. 2012;13(6):519-23.

34. Hamers FP, Lankhorst AJ, van Laar TJ, Veldhuis WB, Gispen WH. Automated quantitative gait analysis during overground locomotion in the rat: its application to spinal cord contusion and transection injuries. J Neurotrauma. 2001;18(2):187-201.

35. Kim KJ, Yoon YW, Chung JM. Comparison of three rodent neuropathic pain models. Exp Brain Res. 1997;113(2):200-6.

36. Smits H, Kleef MV, Honig W, Gerver J, Gobrecht P, Joosten EA. Spinal cord stimulation induces c-Fos expression in the dorsal horn in rats with neuropathic pain after partial sciatic nerve injury. Neurosci Lett. 2009;450(1):70-3.

37. Meuwissen KPV, Gu JW, Zhang TC, Joosten EAJ. Burst Spinal Cord Stimulation in Peripherally Injured Chronic Neuropathic Rats: A Delayed Effect. Pain Pract. 2018.

38. Meuwissen KPV, Gu JW, Zhang TC, Joosten EAJ. Conventional-SCS vs. Burst-SCS and the Behavioral Effect on Mechanical Hypersensitivity in a Rat Model of Chronic Neuropathic Pain: Effect of Amplitude. Neuromodulation. 2018;21(1):19-30.

39. Wang LX, Wang ZJ. Animal and cellular models of chronic pain. Adv Drug Deliv Rev. 2003;55(8):949-65.

40. Koopmans GC, Deumens R, Honig WM, Hamers FP, Steinbusch HW, Joosten EA. The assessment of locomotor function in spinal cord injured rats: the importance of objective analysis of coordination. J Neurotrauma. 2005;22(2):214-25.

41. Koopmans GC, Brans M, Gomez-Pinilla F, Duis S, Gispen WH, Torres-Aleman I, et al. Circulating insulinlike growth factor $I$ and functional recovery from spinal cord injury under enriched housing conditions. Eur $J$ Neurosci. 2006;23(4):1035-46.

42. Hamers FP, Koopmans GC, Joosten EA. CatWalk-assisted gait analysis in the assessment of spinal cord injury. J Neurotrauma. 2006;23(3-4):537-48.

43. Deumens R, Jaken RJ, Marcus MA, Joosten EA. The CatWalk gait analysis in assessment of both dynamic and static gait changes after adult rat sciatic nerve resection. J Neurosci Methods. 2007;164(1):120-30.

44. Neumann M, Wang Y, Kim S, Hong SM, Jeng L, Bilgen M, et al. Assessing gait impairment following experimental traumatic brain injury in mice. J Neurosci Methods. 2009;176(1):34-44.

45. Vlamings R, Visser-Vandewalle V, Koopmans G, Joosten EA, Kozan R, Kaplan S, et al. High frequency stimulation of the subthalamic nucleus improves speed of locomotion but impairs forelimb movement in Parkinsonian rats. Neuroscience. 2007;148(3):815-23.

46. Chiang CY, Sheu ML, Cheng FC, Chen CJ, Su HL, Sheehan J, et al. Comprehensive analysis of neurobehavior associated with histomorphological alterations in a chronic constrictive nerve injury model through use of the CatWalk XT system. J Neurosurg. 2014;120(1):250-62. 
47. Truin $M$, van Kleef $M$, Verboeket $Y$, Deumens $R$, Honig $W$, Joosten EA. The effect of Spinal Cord Stimulation in mice with chronic neuropathic pain after partial ligation of the sciatic nerve. Pain. 2009;145(3):312-8. 48. Koopmans GC, Deumens R, Brook G, Gerver J, Honig WM, Hamers FP, et al. Strain and locomotor speed affect over-ground locomotion in intact rats. Physiol Behav. 2007;92(5):993-1001.

49. Van Zundert J, de Louw AJ, Joosten EA, Kessels AG, Honig W, Dederen PJ, et al. Pulsed and continuous radiofrequency current adjacent to the cervical dorsal root ganglion of the rat induces late cellular activity in the dorsal horn. Anesthesiology. 2005;102(1):125-31.

50. Podhajsky RJ, Sekiguchi Y, Kikuchi S, Myers RR. The histologic effects of pulsed and continuous radiofrequency lesions at 42 degrees $C$ to rat dorsal root ganglion and sciatic nerve. Spine (Phila Pa 1976). 2005;30(9):1008-13.

51. Erdine S, Yucel A, Cimen A, Aydin S, Sav A, Bilir A. Effects of pulsed versus conventional radiofrequency current on rabbit dorsal root ganglion morphology. Eur J Pain. 2005;9(3):251-6.

52. Erdine S, Bilir A, Cosman ER, Cosman ER, Jr. Ultrastructural changes in axons following exposure to pulsed radiofrequency fields. Pain Pract. 2009;9(6):407-17.

53. Huang RY, Liao CC, Tsai SY, Yen CT, Lin CW, Chen TC, et al. Rapid and Delayed Effects of Pulsed Radiofrequency on Neuropathic Pain: Electrophysiological, Molecular, and Behavioral Evidence Supporting LongTerm Depression. Pain Physician. 2017;20(2):E269-E83. 
CHAPTER

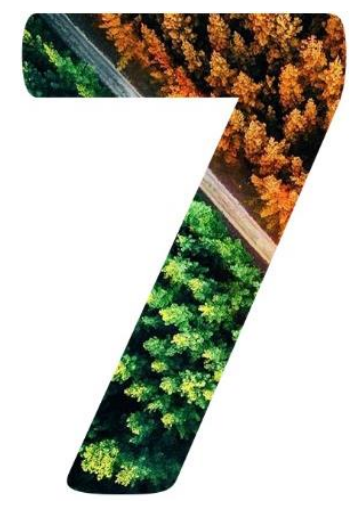

Summary, Discussion, and Conclusion 


\subsection{Primary aim}

The primary aim of this academic thesis was to investigate the efficacy and mechanisms of action of neuromodulation of the DRG in experimental chronic neuropathic pain.

\subsection{Research questions (RQ's).}

Based on the primary aim of this academic thesis, the following research questions were formulated, which will now be discussed:

1. What are the recent developments in the field of SCS in neuropathic pain with focus on novel anatomical targets and paradigms?

2. Does the anti-nociceptive effect of Conventional DRGS and Burst DRGS differ over time in an experimental model of PDPN?

3. What is the effect of Burst DRGS amplitude on mechanical hypersensitivity in an experimental model of PDPN?

4. Does Conventional DRGS reduce intracellular GABA immunoreactivity in DRG somata?

5. Is PRF adjacent to the DRG effective in attenuating mechanical and thermal hypersensitivity and pain-related gait in a rat model of chronic neuropathic pain?

\section{RQ1. What are the recent developments in the field of SCS in neuropathic pain with focus} on novel anatomical targets and paradigms?

The recent developments in SCS for treatment of neuropathic pain, with special focus at new locations and new stimulation paradigms, is reviewed and presented in Chapter 2 "Spinal Cord Stimulation in Chronic Neuropathic Pain: Mechanisms of Action, New Locations, New Paradigms". The aim of this review was to provide a state of the art overview of the field of SCS, in which the efficacy and mechanisms of action of not only Tonic-SCS ( or Con-SCS), but also novel SCS modalities including HF-SCS, Burst-SCS, and DRGS are discussed.

Based on this review a few findings can be summarized: Over the last decade, several new waveforms and anatomical targets have been introduced to the field of SCS to 
improve stimulation efficacy as well as overcome some of the limitations observed with ConSCS. Two prominent examples of novel SCS waveforms that were recently introduced include HF-SCS (1000 Hz-10.000 Hz, $30 \mu$ s pulse width and an amplitude of typically 1-5 mA) [1-5] and BurstDR-SCS (five monophasic spikes administered at $40 \mathrm{~Hz}$ interburst mode and $500 \mathrm{~Hz}$ intraburst frequency, pulse width of $1 \mathrm{~ms}$ and $1 \mathrm{~ms}$ interspike interval, administered with passive charge recovery) [6-8]. These waveforms have the advantage of causing no paresthesias, as they are delivered below perception threshold, and are hypothesized to have different mechanisms of actions as compared to Con-SCS. As the parameter space of these new SCS waveforms has not been fully explored yet, more research is needed in order to fine-tune SCS parameters for specific neuropathic pain indications. For instance, effect differences between passive recharge Burst-SCS (BurstDR-SCS) vs. active recharge Burst-SCS have not been characterized. Besides these novel SCS paradigms, also new anatomical targets, such as DRGS, were recently introduced to the field of SCS $[9,10]$. DRGS has the advantage of reaching and delivering more precise stimulation of difficult-to-reach areas, such as the extremities and the groin. The latter is often difficult, if not impossible to do with SCS using midline lead placement. Additionally, DRGS requires significantly less energy, as there is less CSF present between the lead and the neurological target tissue (the DRG).

Nevertheless, it is important to note that the efficacy and superiority of HF-SCS, Burst-SCS, and DRGS over Con-SCS have only been investigated to a limited extent, and therefore requires more research. Future research should thus be based on an orchestrated interplay between (reproducible) experimental animal studies and well-designed large, (preferably) non-industry sponsored clinical trials. Also the question as to how different SCS paradigms, such as HF-SCS and Burst-SCS, and specific SCS locations, such as DRGS, affect peripheral, spinal, and supraspinal circuits warrants further research. Lastly, there is currently very few research that focusses on combining the advantage of novel SCS waveforms, such as Burst-SCS, and novel anatomical targeting, such as DRGS. The latter combined approach has the potential to not only increase responder rates and analgesic efficacy, but also circumvent some of the other drawbacks related to Con-SCS, including paresthesias, relatively high battery consumption, and lack of specificity. 


\section{RQ2. Does the anti-nociceptive effect of Conventional DRGS and Burst DRGS differ over time in an experimental model of PDPN?}

To answer the second research question, we designed an experimental animal study to test, for the first time, Burst stimulation in combination with DRGS for treating experimental PDPN (see Chapter 3). The experiments as presented in Chapter 3 demonstrate that both Burst-DRGS (five monophasic spikes administered at $40 \mathrm{~Hz}$ interburst mode and $500 \mathrm{~Hz}$ intraburst frequency, pulse width of $1 \mathrm{~ms}$ and $1 \mathrm{~ms}$ interspike interval, administered at $67 \%$ MT with active charge recovery) and Con-DRGS (50 Hz, $250 \mu$ s pulse width, administered at $67 \% \mathrm{MT}$ ) resulted in significant nociception as compared with Sham-DRGS, but at the same time no differences in terms of maximum anti-nociceptive effect were noted. Nevertheless, signs of a residual effect of Burst-DRGS 15 minutes after the stimulator had been turned off, as compared with Con-DRGS, is reported.

The DRGS animal model as used in our study has been utilized previously in a unilateral peripheral nerve injury model of neuropathic pain, and has shown to be an excellent model that mimics features that are typical for clinical DRGS, such as the rapid onset and offset of analgesia [11]. Here, the authors used Con-DRGS $(20 \mathrm{~Hz}, 150 \mu \mathrm{s}$ pulse width, amplitude at $80 \% \mathrm{MT}$ ) and noted similar reductions in von Frey paw withdrawal thresholds in response to DRGS as observed in our study. Importantly, it was also shown that DRGS produces no histological or behavioral signs of injury to the DRG [11].

Since the inception of DRGS in 2011 [9], DRGS (using conventional settings) has been successfully implemented in the clinic for a wide variety of neuropathic pain disorders, including, but not limited to, discogenic low back pain [12, 13], CRPS type I and II [10], postamputation pain [14], and PDPN [15]. The efficacy of DRGS, and the non-inferiority and superiority of DRGS over SCS in patients with CRPS type I and II, has recently been published in the only RCT on DRGS currently available in literature [10]. Here, the percentage of patients receiving $\geq 50 \%$ pain relief and treatment success was significantly increased in the DRG arm (81.2\%) over the SCS arm (55.7\%) at 3 months. Additionally, DRGS demonstrated greater improvements in quality of life and psychological disposition, and DRGS subjects reported 
less postural variation in paresthesia intensity as compared to SCS subjects [10]. Furthermore, the effectiveness of DRGS for PDPN has been published in a retrospective clinical study [15], where PDPN patients reported average reductions in VAS scores of $64 \%$. From this study it was postulated that DRGS might be an effective neuromodulation modality to improve painful symptoms in PDPN [15]. Although the results obtained in these studies are definitely promising, future RCT's, in combination with additional preclinical studies (see Chapter 3), are required.

As presented in Chapter 2, where we provided a broad overview of the field of SCS in the treatment of neuropathic pain and identified some knowledge gaps, one area yet unexplored is the combination of novel anatomical targeting (DRGS) (Chapter 2, section 2.4) and the use of new subperception paradigms (e.g. HF, Burst stimulation) (Chapter 2 section 2.5). To the author's knowledge, prior to the work presented in Chapter 3, only one pilot study has investigated such combination [16]. In this pilot study $(n=5)$, Billet et al. used HFDRGS $(2-10 \mathrm{kHz})$ at either T9 or L2 to treat a mixed cohort of neuropathic pain patients with either FBSS, discopathy or a degenerative SI joint [16]. Although some very promising findings were reported (average reduction in back pain of $61 \%$, average reduction in leg pain of $56 \%$ after 12 weeks), these results should be verified in additional, large-scale RCT's. Interestingly, with the publication of our preclinical studies on Burst-DRGS in PDPN, one clinical study has been published utilizing active recharge Burst-DRGS in neuropathic pain patients [17]. More specific, 32 patients with neuropathic pain were treated with either Con-DRGS or Burst-DRGS using a novel 'transgrade' approach for DRGS implantation. At a mean follow-up of 18 months, $66 \%$ of patients achieved successful DRGS treatment (defined as a Patient Global Impression of Change (PGIC) of 6 or 7, and a decrease in Numeric Rating Scale (NRS-11) of $>2$ ). Importantly, the preferred method of stimulation was found to be the Burst protocol in $78 \%$ of patients [17]. The use of active recharge Burst-DRGS is currently being assessed formally, however, in a RCT that is under way (ClinicalTrials.gov identifier: NCT03318250). It worth noting that both the Con-DRGS and Burst-DRGS waveform as used in the clinical study of Al-Kaisy et al. [17] were delivered subthreshold, while it is likely that the stimulation amplitude of $67 \% \mathrm{MT}$ as used in our animal model is suprathreshold. Differences in terms of 
stimulation amplitude might impact behavioral outcomes in our animals, and pain relief in patients. It is therefore important that the effect of (Burst-)DRGS amplitude on pain outcomes is assessed in additional preclinical (see Chapter 4 ) and clinical studies.

In contrast to the limited literature available on Burst-DRGS, multiple studies have been performed on Burst-SCS targeting the dorsal column (see Chapter 2, section 2.5.3). Here, contradictory findings regarding the superiority of BurstDR-SCS over Con-SCS in treatment of chronic neuropathic pain have been reported. While some studies show a clear advantage of BurstDR-SCS over Con-SCS [18-20], other studies show no difference between the two stimulation modalities in terms of their pain relieving effect [21]. These differences in outcome might be attributed to the different disease indications assessed [18-21]. As discussed in Chapter 2, fundamental differences in terms of working mechanisms may underlie the observed superiority of BurstDR-SCS over Con-SCS in some studies, as BurstDRSCS is thought to specifically stimulate the m-STT in addition to the I-STT, and thereby, much more than Con-SCS, modulates the affective component of the pain experience $[8,19]$. Yet, no significant differences in terms of mechanical hypersensitivity between Con-DRGS and Burst-DRGS were found in our study in Chapter 3. Besides the obvious differences in terms of anatomical target (dorsal column vs. DRG), also the inclusion of only reflex-based tests in the present study might limit our window for detecting differences related to motivationalaffective aspects of pain. It was recently shown in an experimental neuropathic rat model that active recharge Burst-SCS, much more than Con-SCS, affects the cognitive-emotional aspect of pain on the MCAS test, whereas no differences between Burst-SCS and Con-SCS were observed on the reflex-based Von Frey test [22]. Future work on Burst-DRGS should also include operant-like tests like these, in order to see if such differences in terms of cognitive-motivational responses also underlie Burst-DRGS. It is also worth noting that the active recharge Burst waveform used in the present study, albeit monophasic, varies slightly from the commonly clinically used BurstDR waveform as introduced by Dr. De Ridder [19], which is monophasic with a passive recharge balance (see also Chapter 4, and discussion $R Q 3)$. Interestingly, a study on Burst-SCS in a unilateral peripheral nerve injury model of neuropathic pain found Burst-SCS using active recharge to have a delayed onset and a 
delayed carry-over of analgesic effect when compared to Con-SCS [23]. While no differences in terms of a delayed onset of analgesic effect were observed between Con-DRGS and BurstDRGS in our study, Burst-DRGS did appear to show signs of a residual effect at 15 minutes post-stimulation when compared to Con-DRGS. Along these lines, results from a recent randomized controlled trial showed that BurstDR-SCS microdosing, a paradigm that relies on the introduction of stimulation-off phases inbetween stimulation-on phases, is as effective as standard BurstDR-SCS, while having significantly lower battery consumption [24]. Moreover, the analgesic efficacy of BurstDR-SCS microdosing was found to be equal as compared to standard BurstDR-SCS, as measured by rat fMRI brain responses following noxious stimulation [25]. Together, these results strongly indicate a beneficial carry-over effect of Burst stimulation, both when delivered at the dorsal column as well as at the DRG, and suggest that some form of plasticity is induced following each stimulation-on phase. It has recently been shown that the amplitude of spinal neuronal responses in rats can be potentiated for several minutes following a short burst of high-frequency tetanic pulses (555 $\mathrm{Hz}$ ) [26]. As Burst stimulation typically uses a similar intraburst frequency, it is reasonable to assume that Burst stimulation follows a similar mechanism, explaining the persistence of pain relief when the stimulator is off (stimulation-off phase). Furthermore, high frequency stimulation is known to induce long-term potentiation in lamina I of spinal NS projection neurons (see Figure 1, Chapter 2) [27], and signs of short term plasticity have been found in response to electrical stimulation, including those modulated by Burst stimulation [28].

Altogether, our results indicate no differences in maximum effect on mechanical hypersensitivity in PDPN rats between Burst-DRGS and Con-DRGS, although both deliver significant pain relief as compared to Sham-DRGS. Importantly, Burst-DRGS, but not ConDRGS shows signs of a residual effect 15 minutes post-stimulation, which warrants further exploration. Further fine-tuning of parameters related to DRGS is needed and might improve its analgesic properties in the treatment of PDPN and neuropathic pain in general. 


\section{RQ3. What is the effect of Burst DRGS amplitude on mechanical hypersensitivity in an experimental model of PDPN?}

$R Q 3$ has been addressed in Chapter 4. Here, the effect of different BurstDR-DRGS amplitudes on mechanical hypersensitivity in PDPN animals is studied. The results show that BurstDR-DRGS at 33-80\% MT significantly attenuated mechanical hypersensitivity levels both during, and $15 \mathrm{~min}$ after cessation of the stimulation. A nonlinear amplitude-mechanical hypersensitivity relationship is reported, with a calculated optimal BurstDR-DRGS amplitude of $52 \% \mathrm{MT}$ (is equivalent to $\pm 80 \mu \mathrm{A}$ ).

Previous studies indicated that Burst stimulation can be optimized by adjusting relevant stimulation parameters in order to modulate the amount of charge delivered to the nervous system (also see review Chapter 2, section 2.5.3) [29]. In our study, BurstDR-DRGS at 50\% MT and 66\% MT (15 min) and BurstDR-DRGS at 66\% MT (30 min) resulted in highest responder rates, seemingly favoring amplitudes of $50-66 \%$ over the higher $(80 \% \mathrm{MT})$ and lower $(0-33 \% \mathrm{MT})$ stimulation amplitudes. Previous work on SCS suggests the sensation threshold, defined as the amplitude at which animals start to show signs of disturbance of their normal behavior (overt behavioral responses including attending to hindlimb or lower body, exploratory behavior, grooming behavior, or alertness directly related to stimulation onset $[30,31])$ to be around $50 \% \mathrm{MT}[31]$. One could therefore speculate that the gradual decrease in therapeutic efficacy beyond this 50\% MT optimum is due to the perception of sensory, potential uncomfortable, sensations by the animal. Previous work on Burst-SCS (active recharge) in an experimental animal model of neuropathic pain has shown a similar nonlinear course between amplitude and behavioral outcome with an optimum at 50\% MT [32]. Interestingly, this optimal effect at 50\% MT using active recharge Burst-SCS was shown to rapidly decline when this optimal amplitude was surpassed [32]. This is in line with the findings of Courtney et al. who demonstrated that the absolute therapeutic window of BurstDR-SCS in terms of amplitude is considerably smaller when compared to Con-SCS in patients with intractable chronic pain [33]. In contrast, the BurstDR-DRGS paradigm as used in the present study demonstrated a larger optimal therapeutic window (50-66\% MT). 
The combination of our findings (Chapter 4) and data from literature [32] suggest that Burst stimulation is effective at lower amplitudes relative to the observed MT as compared to conventional stimulation. The combination of this relatively low optimal Burst stimulation amplitude, both in DRGS and SCS studies, together with the previously described increased carry-over effect of Burst stimulation, could have important implications for optimal stimulation delivery as well as battery life of the IPG in clinical practice.

Taken all parameters together, stimulation as with DRGS or SCS can be characterized as 'charge over time'. The same charge per time unit, or charge per second (CPS), can be achieved using various dosing strategies, including, but not limited to, conventional stimulation, HF stimulation, and Burst stimulation. Whereas conventional stimulation typically is characterized by a high amplitude (and a relatively low frequency) to achieve the desired CPS, dosing strategies that utilize higher frequencies and/or wider pulse widths (such as $\mathrm{HF}$ and Burst stimulation) require less amplitude to do so. In the field of neuromodulation and pain, different concepts are currently hypothesized to determine stimulation outcome. One concept is related to the idea that the therapeutic efficacy in terms of pain relief merely depends on the amount of CPS delivered to the nociceptive system (which has a certain optimum), and that use of two different dosing strategies should result in similar pain relief, given that the CPS is equal [34]. The strength-duration curve as described by Miller et al. demonstrates that wider pulse widths require less amplitude to activate a neuron, whereas smaller pulse widths require more amplitude [34]. Since amplitude generally impacts the amount of fibers recruited, and thereby increases or decreases in perceived paresthesias, a paradigm with a high duty cycle (defined as duration of active stimulation/total duration), such as Burst stimulation, requires less amplitude while still delivering the same amount of CPS, and overcoming paresthesias. On the other hand, another concept currently discussed in the field of SCS and pain relief is related to the idea that it is not the CPS that determines stimulation efficacy, but instead individual parameters such as amplitude, pulse width or frequencies, irrespective of their combined total charge [34]. 
Interestingly, an additional aspect related to the stimulation paradigm, charge delivery and pain relief that was recently introduced by De Ridder and colleagues, is the recharge phase [35]. Each delivered pulse, characterized by a specific amount of current (mA) for a specific amount of time (pulse width), is followed by this so-called 'recharge phase'. A recharge phase consists of a flow of current in the opposite direction, in order to balance the charge delivered to the system and serve as a safeguard against overstimulation and tissue damage. This recharge phase can either be active or passive in nature; in most neuromodulation systems, charge balancing occurs after each individual pulse in a passive manner [35]. The Burst stimulation paradigm introduced into the field by de Ridder et al. (BurstDR stimulation) [19], and as used in our study (Chapter 4), is a passive recharge form of Burst stimulation where the built up charge period is discharged during the interburst period. Passive recharge Burst stimulation has been hypothesized to mimic natural neuronal burst firing in the thalamus, where the 'plateau' that occurs during the active phase of the Burst and that is followed by a quiescent period called the 'silent phase' is generated by calcium influx via T-type calcium channels, generating low threshold $\mathrm{Ca}^{2+}$ potentials upon the crest of which sodium and potassium channel-mediated action potentials fire, creating lowthreshold bursts [35]. On the other hand, with use of active recharge Burst, charge balance is maintained during the intraburst period. To date, only one small pilot study has directly compared active vs. passive (BurstDR) recharge Burst stimulation [36]. In this study, the authors studied both evoked and spontaneous neuronal firing rates in the dorsal horn of the spinal cord, and compared active vs. passive recharge Burst-SCS in rats that underwent cervical nerve root compression. Here it was found that passive recharge Burst-SCS (BurstDR), but not active recharge Burst-SCS, reduces neuronal firing rats in the dorsal horn upon the application of a noxious stimulus [36]. However, one should not neglect the fact that this was a pilot study measuring only a very limited amount of neurons $(n=9)$, and that no behavioral assessments were performed. As such, no definitive conclusion regarding a possible superiority of passive vs. active recharge Burst-SCS related to impact on pain relief can be made. Future preclinical as well as clinical studies should focus on comparing these 
two different types of Burst SCS and charge recovery, and assess if and how they may differentially affect the nociceptive system and cause pain relief.

Altogether, our findings suggest a nonlinear relationship between BurstDR-DRGS amplitude and mechanical hypersensitivity in a rat model of PDPN. Basic animal studies on the mechanisms of action of Burst-DRGS and the role of various stimulation parameters including the type of charge recovery are needed to further optimize pain relief.

\section{RQ4. Does Conventional DRGS reduce intracellular GABA immunoreactivity in DRG somata?}

In order to answer the fourth research question, we studied the effect of Con-DRGS on local intracellular GABA immunoreactivity in DRG somata in Chapter 5. From this detailed quantitative immunocytochemical study it is concluded that Con-DRGS does not alter the average intracellular GABA immunofluorescence staining intensity in DRG sensory neurons of animals which showed significant pain reduction. No cell size specific effect was noted as the intensity of GABA-immunoreactivity in cell somata of small, medium ("nociceptive") or large ("non-nociceptive") sensory neurons was unaffected by Con-DRGS.

As described in Chapter 2, section 2.3.1, GABA is thought to play a pivotal role in the mechanism underlying Con-SCS of the dorsal columns. Con-SCS acts antidromically via stimulating the fast conducting, myelinated, touch-affiliated ("non-nociceptive") A $\beta$-fibers located in the superficial dorsal column. With this mode of action Con-SCS is able to close the 'spinal pain gate' by activating GABAergic inhibitory interneurons located in the upper laminae of the dorsal horn (see Figure 1, Chapter 2). These inhibitory interneurons, upon activation, then release GABA, which blocks the incoming nociceptive input from the smaller, unmyelinated C-fibers. In the field of pain this is often simply referred to as "fast blocks slow" [37]. Indeed, previous work has demonstrated that intracellular GABA immunoreactivity in the dorsal horn is increased in chronic neuropathic rats [38], but is subsequently decreased after treatment with Con-SCS $[39,40]$. At the same time, extracellular levels of GABA are increased following Con-SCS, as measured by microdialysis [41-43]. Moreover, the analgesic 
properties of Con-SCS can be blocked [40,41] or potentiated [44, 45] by administering GABАв-receptor antagonists or agonists, respectively.

DRGS differs from SCS of the dorsal columns in its ability, at least theoretically, to target multiple types of sensory fibers, as the somata of all sensory modalities (nonnociceptive $A \beta$, and nociceptive $A \delta$ and $C$ ) reside in the DRG. While in theory DRGS could stimulate $A \beta$ afferents, something that is supported by a recent computational study by Graham and colleagues [46], it is unlikely that this results in GABAergic spinal gating mechanisms in a similar way as with Con-SCS. Recent findings, based on quantitative immunocytochemical analysis showed that Con-DRGS does not affect intensity of GABA immunoreactivity in the spinal cord dorsal horn [47]. Interestingly, a recent study by Du et al. reported the presence of an extensive GABAergic communication network inside the DRG itself. The authors suggested the presence of a "second pain gate", and further showed that DRG somata of all sizes (small and medium sized nociceptive as well as large sized nonnociceptive neurons) can release GABA upon depolarization [48]. This led us to hypothesize that Con-DRGS might exert its analgesic action by engaging this second pain gate located in the DRG.

Clearly, we were not able to confirm this hypothesis, as the average intensity of GABA immunofluorescence of cell somata in DRG's treated with Con-DRGS did not significantly differ from Sham-treated DRG's. An interesting observation was the generally higher GABA immunofluorescence noted in small-sized somata $(12-26 \mu \mathrm{m})$ as compared to the medium $(26-40 \mu \mathrm{m})$ and large-sized $(26-40 \mu \mathrm{m})$ somata, suggesting the higher importance of GABAergic signaling in these cells which presumably are nociceptive. A critical note needs to be made as one should be careful when extrapolating the diameter ranges as being absolutely representative for pure $C, A \delta$, or $A \beta$ sensory neuron populations. Villiere et al. showed that the $C, A \delta$, and $A \beta$ type superposes the soma diameter [49]. Additionally, Lee and colleagues found that the soma size of these intermediate cells is not correlated with conduction velocity because these cells appear both with myelinated as well as unmyelinated axons [50]. 
Our results in Chapter 5 strongly suggest that Con-DRGS does not modulate the "second pain gate in the DRG". Hence, the analgesic mechanism of action of DRGS might simply not be GABA mediated, but other, non-GABA mediated mechanisms of action are likely to be involved. Over the years, few studies have investigated the mechanism of action of Con-DRGS. A study by Koopmeiners et al. [51] and Chao et al. [52] found Con-DRGS to block the conduction of afferent impulse trains, specifically in nociceptive sensory afferents. The unique pseudo-unipolar design and the T-junction of the DRG may act as an electrophysiological low-pass filter for electrical stimuli travelling from the periphery to the spinal cord [53]. Furthermore, it was shown by fMRI that Con-DRGS is capable of attenuating BOLD signals in brain regions that are considered part of the pain matrix, like the contralateral thalamic VPL/VPM nuclei, and cortical S1 and S2 [54]. Lastly, a recent literature review by Chapman et al. [55] suggests that DRGS, especially at low frequencies (typically well below $20 \mathrm{~Hz}$ ), has the ability to lead to dorsal horn inhibition via distinct mechanisms, including inhibition of dorsal horn excitatory interneurons through spinal opioid system activation $(1 \mathrm{~Hz})[56,57]$, and the activation of low-threshold mechanoreceptors (LTMR's), leading to the release of endorphins and/or enkephalins in the spinal cord dorsal horn [57-60]. Along these lines, a study by Koetsier et al. indeed found very low frequency DRGS $(1 \mathrm{~Hz})$ to have a significantly longer wash-out period as compared to $20 \mathrm{~Hz}$ and $1000 \mathrm{~Hz}$ DRGS, which might be due to activation of one of these pathways [61].

In order to further understand the mechanisms underlying Con-DRGS and pain relief, our future research agenda should focus at non-GABAergic mechanisms either at the spinal dorsal horn, supraspinal levels, and/or in the DRG itself. Nevertheless, local GABAergic signaling inside the DRG might still underlie the pain relieving effect of novel DRGS paradigms (such as Burst- or HF-DRGS).

RQ5. Is PRF adjacent to the DRG effective in attenuating mechanical and thermal hypersensitivity and pain-related gait in a rat model of chronic neuropathic pain?

In order to answer RQ5, an animal experiment was designed to test the efficacy of PRF applied adjacent to the L5 DRG on not only mechanical and thermal hypersensitivity, but 
also pain-related gait (see Chapter 6). Here it is shown that treatment with 2x 180s PRF (pulse frequency $=2 \mathrm{~Hz}$, pulse width $=20 \mathrm{~ms}$, output voltage $=45 \mathrm{~V}$, and maximum temperature $=42^{\circ} \mathrm{C}$ (kept $<42^{\circ} \mathrm{C}$ by varying pulse width) significantly attenuated PSNL-induced mechanical and thermal hypersensitivity, whereas no effect was observed on pain-related gait.

With our study, we confirm previous findings (reviewed in [62]) related to the effect of PRF on mechanical and thermal hypersensitivity in chronic neuropathic pain, something which is important when establishing a reproducible and valid animal model for studying the effects of PRF. Nevertheless, comparing outcomes between experimental studies in animals remains difficult, as studies are subjected to great diversity in terms of not only the animal models used, but also PRF location and PRF duration. Our PRF protocol of 2x 180s applied adjacent to the DRG in chronic neuropathic rats showed profound attenuation of mechanical and thermal hypersensitivity for up to 15 days. Interestingly, a study by Perret et al. showed that animals that received PRF treatment adjacent to the L5 DRG exhibited better recovery and showed larger effect sizes than sham treated animals for a period up to no less than 50 days post-PRF treatment [63]. Indeed, both our Von Frey (mechanical hypersensitivity) and Hargreaves (thermal hypersensitivity) data show no signs of attenuation in terms of PRF treatment efficacy at post-PRF day 15 , making it very likely that the pain relieving effect of PRF would have persisted after 15 days. Thus, our findings are in line with those reported by Perret and colleagues [63]. The long duration of pain relieving effect with PRF is in major contrast to the transient effect as noted with DRGS (see Chapters 3 and 4). Whereas DRGS results in a near complete attenuation of mechanical hypersensitivity (based on optimal amplitude of stimulation used; see Chapter 4), this effect fades away within 15-30 minutes post-stimulation (differences in wash-out related to SCS paradigm used; see Chapter 3 ). The effect of PRF lasts for weeks if not months, but the effect on attenuation of mechanical hypersensitivity per se is limited and much milder (recovery to $40-50 \%$ of pre-PSNL baseline). These differences in terms of the onset and amount of pain relief of these two neuromodulatory treatments (i.e. PRF and DRGS) require a neurobiological and mechanistic understanding. The mechanism of action underlying DRGS is thought to mainly act by inducing a temporary conduction block through the C-type T-junction located in the DRG 
itself $[51,53]$ (see Chapter 2, Figure 1). This conduction block might then act as an impediment or low-pass filter to electrical impulses traveling from the peripheral nociceptor to the spinal cord [53]. In contrast, PRF has been reported to affect the release of both excitatory [64] and inhibitory [65] neurotransmitters and the activation of microglia [66, 67] in the dorsal horn of the spinal cord. More importantly, although the general consensus is that PRF relies on a neuromodulatory effect, the factor neurodestruction, albeit on a microscopic level, cannot be ruled out. Multiple studies have shown that PRF adjacent to the DRG can cause small histological and morphological changes, like enlarged endoplasmic reticulum cisterns and increased number of cytoplasmic vacuoles. These changes seem to be most pronounced in C-fibers and are much milder when compared to RF-induced morphological changes [68-70]. These very small morphological and histological changes might be just enough to modulate axonal transport of proteins important in the development and maintenance of chronic neuropathic pain and central sensitization, such as BDNF, substance-P, and glutamate (see Chapter 1, section 1.2) [71]. Additionally, a recent study found that low-voltage bipolar PRF alleviates long-lasting neuropathic pain by selectively and persistently modulating C-fiber-mediated spinal nociceptive hypersensitivity and long-term depression [72]. Such 'structural' changes would then provide a reasonable explanation for the maintained and long-lasting pain reductions observed in clinical and preclinical PRF studies.

Studying behavioral effects (short or long-lasting) is still an issue in the experimental pain field and may make the impact of pain relieving effects and the interpretation and translation of findings to the clinic very difficult. To date, reflex-based outcome measures such as mechanical hypersensitivity (e.g. Von Frey test) and thermal hypersensitivity (e.g. Hargreaves test) are considered to be the golden standard in experimental pain research. However, as these tests are rather subjective and rely on evoked nociception instead of spontaneous pain, they are not optimal.

Besides reflex-based tests like the Von Frey and Hargreaves assay, several attempts have been undertaken to measure behavioral changes in animal models, as an indirect 
measure of pain. Over the years, CatWalk gait analysis has been demonstrated to be one the most complete and objective methods of measuring gait in experimental animal research $[73,74]$. The CatWalk system has been used in experimental animal research for a wide variety of neurological disorders, ranging from spinal cord injury [75, 76] and peripheral nerve injury [77, 78] to traumatic brain injury [79] and Parkinson's disease [80]. In our study, CatWalk gait analysis of animals that underwent PSNL showed significant decreases over time for all selected CatWalk parameters. Interestingly, our results in Chapter 6 showed a gradual normalization of Catwalk parameters over time, possibly hinting to development of adaptation behavior in the animals, and something that is in line with literature [77]. We were not able to detect any behavioral effects of PRF on CatWalk pain-induced gait parameters. At the same time a profound effect of PRF treatment was observed on mechanical and thermal hypersensitivity as measured by the von Frey and Hargreaves test. In line with this, a study by Truin and colleagues, using the same animal model, showed no effect of SCS on any of the measured CatWalk gait parameters, but did show profound increases in Von Frey values [81]. Although spinal cord stimulation and pulsed radiofrequency treatment are fundamentally different in their properties and proposed working mechanisms, the fact that two of the most widely adopted interventional pain treatment approaches seem to not alter pain-induced gait, while at the same time attenuating mechanical and thermal hypersensitivity in chronic neuropathic animals, strongly suggests that CatWalk gait analysis does not allow to detect or analyze behavioral effects of interventional treatment approaches in the chronic experimental PSNL model.

To conclude, PRF adjacent to the L5 DRG resulted in longer-lasting, but milder attenuation of chronic neuropathic pain, as compared with DRGS. Use of CatWalk based gait analysis did not allow the detection of PRF-induced changes. Future research should focus on elucidating the mechanisms underlying PRF treatment and optimization of the stimulation paradigm. 


\subsection{Conclusion}

In summary, the results presented in this academic thesis show that modulation of the DRG via either Con-DRGS or PRF results in effective pain relief in two well-established rodent models of chronic neuropathic pain. The fundamental differences in terms of efficacy and duration of pain relief between Con-DRGS (efficacy: near complete pain reduction; short, transient effect) and PRF (efficacy: max 40-50\% pain reduction; long duration effect) are discussed, and likely depend on different mechanisms of action. Further fundamental, neurobiological studies are needed. The introduction of novel DRGS stimulation paradigms, such as Burst-DRGS, have the potential to improve the therapeutic efficacy of DRGS. 


\subsection{References}

1. Van Buyten JP, Al-Kaisy A, Smet I, Palmisani S, Smith T. High-frequency spinal cord stimulation for the treatment of chronic back pain patients: results of a prospective multicenter European clinical study. Neuromodulation. 2013;16(1):59-65; discussion -6.

2. Song Z, Meyerson BA, Linderoth B. High-Frequency (1 kHz) Spinal Cord Stimulation-Is Pulse Shape Crucial for the Efficacy? A Pilot Study. Neuromodulation. 2015;18(8):714-20.

3. Shechter R, Yang F, Xu Q, Cheong YK, He SQ, Sdrulla A, et al. Conventional and kilohertz-frequency spinal cord stimulation produces intensity- and frequency-dependent inhibition of mechanical hypersensitivity in a rat model of neuropathic pain. Anesthesiology. 2013;119(2):422-32.

4. Annemans L, Van Buyten JP, Smith T, Al-Kaisy A. Cost effectiveness of a novel $10 \mathrm{kHz}$ high-frequency spinal cord stimulation system in patients with failed back surgery syndrome (FBSS). J Long Term Eff Med Implants. 2014;24(2-3):173-83.

5. Al-Kaisy A, Van Buyten JP, Smet I, Palmisani S, Pang D, Smith T. Sustained effectiveness of $10 \mathrm{kHz}$ highfrequency spinal cord stimulation for patients with chronic, low back pain: 24-month results of a prospective multicenter study. Pain Med. 2014;15(3):347-54.

6. Kriek N, Groeneweg G, Huygen FJ. Burst Spinal Cord Stimulation in a Patient with Complex Regional Pain Syndrome: A 2-year Follow-Up. Pain Pract. 2015;15(6):E59-64.

7. De Ridder D, Vanneste S, Plazier M, van der Loo E, Menovsky T. Burst spinal cord stimulation: toward paresthesia-free pain suppression. Neurosurgery. 2010;66(5):986-90.

8. De Ridder D, Vanneste S. Burst and Tonic Spinal Cord Stimulation: Different and Common Brain Mechanisms. Neuromodulation. 2016;19(1):47-59.

9. Liem L, Russo M, Huygen FJ, Van Buyten JP, Smet I, Verrills P, et al. A multicenter, prospective trial to assess the safety and performance of the spinal modulation dorsal root ganglion neurostimulator system in the treatment of chronic pain. Neuromodulation. 2013;16(5):471-82; discussion 82.

10. Deer TR, Levy RM, Kramer J, Poree L, Amirdelfan K, Grigsby E, et al. Dorsal root ganglion stimulation yielded higher treatment success rate for complex regional pain syndrome and causalgia at 3 and 12 months: a randomized comparative trial. Pain. 2017;158(4):669-81.

11. Pan B, Yu H, Fischer GJ, Kramer JM, Hogan QH. Dorsal Root Ganglionic Field Stimulation Relieves Spontaneous and Induced Neuropathic Pain in Rats. J Pain. 2016;17(12):1349-58.

12. Huygen F, Liem L, Cusack W, Kramer J. Stimulation of the L2-L3 Dorsal Root Ganglia Induces Effective Pain Relief in the Low Back. Pain Pract. 2018;18(2):205-13.

13. Kallewaard JW, Edelbroek C, Terheggen M, Raza A, Geurts JW. A Prospective Study of Dorsal Root Ganglion Stimulation for Non-Operated Discogenic Low Back Pain. Neuromodulation. 2020;23(2):196-202.

14. Eldabe S, Burger K, Moser H, Klase D, Schu S, Wahlstedt A, et al. Dorsal Root Ganglion (DRG) Stimulation in the Treatment of Phantom Limb Pain (PLP). Neuromodulation. 2015;18(7):610-6; discussion 6-7.

15. Eldabe S, Espinet A, Wahlstedt A, Kang P, Liem L, Patel NK, et al. Retrospective Case Series on the Treatment of Painful Diabetic Peripheral Neuropathy With Dorsal Root Ganglion Stimulation. Neuromodulation. 2018;21(8):787-92.

16. Billet B, Hanssens K, De Coster O, Nagels W, Weiner RL, Wynendaele R, et al. Wireless high-frequency dorsal root ganglion stimulation for chronic low back pain: A pilot study. Acta Anaesthesiol Scand. 2018.

17. Al-Kaisy A, Royds J, Costanzi M, Racz G, Wesley S, Palmisani S, et al. Effectiveness of "Transgrade" Epidural Technique for Dorsal Root Ganglion Stimulation. A Retrospective, Single-Center, Case Series for Chronic Focal Neuropathic Pain. Pain Physician. 2019;22(6):601-11.

18. De Ridder D, Lenders MW, De Vos CC, Dijkstra-Scholten C, Wolters R, Vancamp T, et al. A 2-center comparative study on tonic versus burst spinal cord stimulation: amount of responders and amount of pain suppression. Clin J Pain. 2015;31(5):433-7.

19. De Ridder D, Plazier M, Kamerling N, Menovsky T, Vanneste S. Burst spinal cord stimulation for limb and back pain. World Neurosurg. 2013;80(5):642-9 e1.

20. de Vos CC, Bom MJ, Vanneste S, Lenders MW, de Ridder D. Burst spinal cord stimulation evaluated in patients with failed back surgery syndrome and painful diabetic neuropathy. Neuromodulation. 2014;17(2):152-9.

21. Kriek N, Groeneweg JG, Stronks DL, de Ridder D, Huygen FJ. Preferred frequencies and waveforms for spinal cord stimulation in patients with complex regional pain syndrome: A multicentre, double-blind, randomized and placebo-controlled crossover trial. Eur J Pain. 2017;21(3):507-19. 
22. Meuwissen KPV, van Beek M, Joosten EAJ. Burst and Tonic Spinal Cord Stimulation in the Mechanical Conflict-Avoidance System: Cognitive-Motivational Aspects. Neuromodulation. 2019.

23. Meuwissen KPV, Gu JW, Zhang TC, Joosten EAJ. Burst Spinal Cord Stimulation in Peripherally Injured Chronic Neuropathic Rats: A Delayed Effect. Pain Pract. 2018;18(8):988-96.

24. Vesper J, Slotty P, Schu S, Poeggel-Kraemer K, Littges H, Van Looy P, et al. Burst SCS Microdosing Is as Efficacious as Standard Burst SCS in Treating Chronic Back and Leg Pain: Results From a Randomized Controlled Trial. Neuromodulation. 2019;22(2):190-3.

25. Saber M SD TJ, Khan Z. Rat FMRI brain responses to noxious stimulation during tonic, burst, and burstmicrodosing spinal cord stimulation. Poster, International Neuromodulation Society 2019.

26. Lloyd DP. Post-tetanic potentiation of response in monosynaptic reflex pathways of the spinal cord. J Gen Physiol. 1949;33(2):147-70.

27. Ikeda H, Heinke B, Ruscheweyh R, Sandkuhler J. Synaptic plasticity in spinal lamina I projection neurons that mediate hyperalgesia. Science. 2003;299(5610):1237-40.

28. Shyu BC, Vogt BA. Short-term synaptic plasticity in the nociceptive thalamic-anterior cingulate pathway. Mol Pain. 2009;5:51.

29. Crosby ND, Goodman Keiser MD, Smith JR, Zeeman ME, Winkelstein BA. Stimulation parameters define the effectiveness of burst spinal cord stimulation in a rat model of neuropathic pain. Neuromodulation. 2015;18(1):1-8; discussion

30. Koyama S, Xia J, Leblanc BW, Gu JW, Saab CY. Sub-paresthesia spinal cord stimulation reverses thermal hyperalgesia and modulates low frequency EEG in a rat model of neuropathic pain. Sci Rep. 2018;8(1):7181.

31. Song Z, Viisanen H, Meyerson BA, Pertovaara A, Linderoth B. Efficacy of kilohertz-frequency and conventional spinal cord stimulation in rat models of different pain conditions. Neuromodulation. 2014;17(3):22634; discussion 34-5.

32. Meuwissen KPV, Gu JW, Zhang TC, Joosten EAJ. Conventional-SCS vs. Burst-SCS and the Behavioral Effect on Mechanical Hypersensitivity in a Rat Model of Chronic Neuropathic Pain: Effect of Amplitude. Neuromodulation. 2018;21(1):19-30.

33. Courtney P, Espinet A, Mitchell B, Russo M, Muir A, Verrills P, et al. Improved Pain Relief With Burst Spinal Cord Stimulation for Two Weeks in Patients Using Tonic Stimulation: Results From a Small Clinical Study. Neuromodulation. 2015;18(5):361-6.

34. Miller JP, Eldabe S, Buchser E, Johanek LM, Guan Y, Linderoth B. Parameters of Spinal Cord Stimulation and Their Role in Electrical Charge Delivery: A Review. Neuromodulation. 2016;19(4):373-84.

35. De Ridder D, Vancamp T, Falowski SM, Vanneste S. All bursts are equal, but some are more equal (to burst firing): burstDR stimulation versus Boston burst stimulation. Expert Rev Med Devices. 2020.

36. Kent AR, Weisshaar CL, Venkatesan L, Winkelstein BA. Burst \& High-Frequency Spinal Cord Stimulation Differentially Effect Spinal Neuronal Activity After Radiculopathy. Ann Biomed Eng. 2020;48(1):112-20.

37. Noordenbos. Pain. Elsevier, Amsterdam. 1959.

38. Janssen SP, Truin $M$, Van Kleef $M$, Joosten EA. Differential GABAergic disinhibition during the development of painful peripheral neuropathy. Neuroscience. 2011;184:183-94.

39. Janssen SP, Gerard S, Raijmakers ME, Truin M, Van Kleef M, Joosten EA. Decreased intracellular GABA levels contribute to spinal cord stimulation-induced analgesia in rats suffering from painful peripheral neuropathy: the role of KCC2 and GABA(A) receptor-mediated inhibition. Neurochem Int. 2012;60(1):21-30.

40. Meuwissen KPV, de Vries LE, Gu JW, Zhang TC, Joosten EAJ. Burst and Tonic Spinal Cord Stimulation Both Activate Spinal GABAergic Mechanisms to Attenuate Pain in a Rat Model of Chronic Neuropathic Pain. Pain Pract. 2020;20(1):75-87.

41. Cui JG, O'Connor WT, Ungerstedt U, Linderoth B, Meyerson BA. Spinal cord stimulation attenuates augmented dorsal horn release of excitatory amino acids in mononeuropathy via a GABAergic mechanism. Pain. 1997;73(1):87-95.

42. Linderoth B, Stiller CO, Gunasekera L, O'Connor WT, Ungerstedt U, Brodin E. Gamma-aminobutyric acid is released in the dorsal horn by electrical spinal cord stimulation: an in vivo microdialysis study in the rat. Neurosurgery. 1994;34(3):484-8; discussion 8-9.

43. Stiller CO, Cui JG, O'Connor WT, Brodin E, Meyerson BA, Linderoth B. Release of gamma-aminobutyric acid in the dorsal horn and suppression of tactile allodynia by spinal cord stimulation in mononeuropathic rats. Neurosurgery. 1996;39(2):367-74; discussion 74-5. 
44. Cui JG, Meyerson BA, Sollevi A, Linderoth B. Effect of spinal cord stimulation on tactile hypersensitivity in mononeuropathic rats is potentiated by simultaneous $G A B A(B)$ and adenosine receptor activation. Neurosci Lett. 1998;247(2-3):183-6.

45. Lind G, Schechtmann G, Winter J, Meyerson BA, Linderoth B. Baclofen-enhanced spinal cord stimulation and intrathecal baclofen alone for neuropathic pain: Long-term outcome of a pilot study. Eur J Pain. 2008;12(1):1326.

46. Graham RD, Bruns TM, Duan B, Lempka SF. Dorsal root ganglion stimulation for chronic pain modulates Abeta-fiber activity but not C-fiber activity: A computational modeling study. Clin Neurophysiol. 2019;130(6):94151.

47. Koetsier E, Franken G, Debets J, Heijmans L, van Kuijk SMJ, Linderoth B, et al. Mechanism of dorsal root ganglion stimulation for pain relief in painful diabetic polyneuropathy is not dependent on GABA release in the dorsal horn of the spinal cord. CNS Neurosci Ther. 2019.

48. Du X, Hao H, Yang Y, Huang S, Wang C, Gigout S, et al. Local GABAergic signaling within sensory ganglia controls peripheral nociceptive transmission. J Clin Invest. 2017;127(5):1741-56.

49. Villiere V, McLachlan EM. Electrophysiological properties of neurons in intact rat dorsal root ganglia classified by conduction velocity and action potential duration. J Neurophysiol. 1996;76(3):1924-41.

50. Lee KH, Chung K, Chung JM, Coggeshall RE. Correlation of cell body size, axon size, and signal conduction velocity for individually labelled dorsal root ganglion cells in the cat. J Comp Neurol. 1986;243(3):335-46.

51. Koopmeiners AS, Mueller S, Kramer J, Hogan QH. Effect of electrical field stimulation on dorsal root ganglion neuronal function. Neuromodulation. 2013;16(4):304-11; discussion 10-1.

52. Chao D, Zhang Z, Mecca CM, Hogan QH, Pan B. Analgesic dorsal root ganglionic field stimulation blocks conduction of afferent impulse trains selectively in nociceptive sensory afferents. Pain. 2020.

53. Krames ES. The dorsal root ganglion in chronic pain and as a target for neuromodulation: a review. Neuromodulation. 2015;18(1):24-32; discussion

54. Pawela CP, Kramer JM, Hogan QH. Dorsal root ganglion stimulation attenuates the BOLD signal response to noxious sensory input in specific brain regions: Insights into a possible mechanism for analgesia. Neuroimage. 2017;147:10-8.

55. Chapman KB, Groenen PS, Vissers KC, van Helmond N, Stanton-Hicks MD. The Pathways and Processes Underlying Spinal Transmission of Low Back Pain: Observations From Dorsal Root Ganglion Stimulation Treatment. Neuromodulation. 2020.

56. Ikeda H, Asai T, Randic M, Murase K. Robust suppression of afferent-induced excitation in the rat spinal dorsal horn after conditioning low-frequency stimulation. J Neurophysiol. 1999;82(4):1957-64.

57. Sandkuhler J, Chen JG, Cheng G, Randic M. Low-frequency stimulation of afferent Adelta-fibers induces long-term depression at primary afferent synapses with substantia gelatinosa neurons in the rat. J Neurosci. 1997;17(16):6483-91.

58. Arcourt A, Gorham L, Dhandapani R, Prato V, Taberner FJ, Wende H, et al. Touch Receptor-Derived Sensory Information Alleviates Acute Pain Signaling and Fine-Tunes Nociceptive Reflex Coordination. Neuron. 2017;93(1):179-93.

59. Lu Y, Perl ER. A specific inhibitory pathway between substantia gelatinosa neurons receiving direct Cfiber input. J Neurosci. 2003;23(25):8752-8.

60. Narikawa K, Furue H, Kumamoto E, Yoshimura M. In vivo patch-clamp analysis of IPSCs evoked in rat substantia gelatinosa neurons by cutaneous mechanical stimulation. J Neurophysiol. 2000;84(4):2171-4.

61. Koetsier E, Franken G, Debets J, van Kuijk SMJ, Linderoth B, Joosten EA, et al. Dorsal Root Ganglion Stimulation in Experimental Painful Diabetic Polyneuropathy: Delayed Wash-Out of Pain Relief After Low-Frequency $(1 \mathrm{~Hz})$ Stimulation. Neuromodulation. 2020;23(2):177-84.

62. Vuka I, Vucic K, Repic T, Ferhatovic Hamzic L, Sapunar D, Puljak L. Electrical Stimulation of Dorsal Root Ganglion in the Context of Pain: A Systematic Review of In Vitro and In Vivo Animal Model Studies. Neuromodulation. 2018;21(3):213-24.

63. Perret DM, Kim DS, Li KW, Sinavsky K, Newcomb RL, Miller JM, et al. Application of pulsed radiofrequency currents to rat dorsal root ganglia modulates nerve injury-induced tactile allodynia. Anesth Analg. 2011;113(3):6106.

64. Yang $\mathrm{CH}$, Chen $\mathrm{KH}$, Huang HW, Sheen-Chen SM, Lin CR. Pulsed radiofrequency treatment attenuates increases in spinal excitatory amino acid release in rats with adjuvant-induced mechanical allodynia. Neuroreport. $2013 ; 24(8): 431-6$. 
65. Hagiwara S, Iwasaka H, Takeshima N, Noguchi T. Mechanisms of analgesic action of pulsed radiofrequency on adjuvant-induced pain in the rat: roles of descending adrenergic and serotonergic systems. Eur J Pain. 2009;13(3):249-52.

66. Cho HK, Cho YW, Kim EH, Sluijter ME, Hwang SJ, Ahn SH. Changes in pain behavior and glial activation in the spinal dorsal horn after pulsed radiofrequency current administration to the dorsal root ganglion in a rat model of lumbar disc herniation: laboratory investigation. J Neurosurg Spine. 2013;19(2):256-63.

67. Park HW, Ahn SH, Son JY, Kim SJ, Hwang SJ, Cho YW, et al. Pulsed radiofrequency application reduced mechanical hypersensitivity and microglial expression in neuropathic pain model. Pain Med. 2012;13(9):1227-34.

68. Erdine S, Bilir A, Cosman ER, Cosman ER, Jr. Ultrastructural changes in axons following exposure to pulsed radiofrequency fields. Pain Pract. 2009;9(6):407-17.

69. Erdine S, Yucel A, Cimen A, Aydin S, Sav A, Bilir A. Effects of pulsed versus conventional radiofrequency current on rabbit dorsal root ganglion morphology. Eur J Pain. 2005;9(3):251-6.

70. Podhajsky RJ, Sekiguchi Y, Kikuchi S, Myers RR. The histologic effects of pulsed and continuous radiofrequency lesions at 42 degrees $C$ to rat dorsal root ganglion and sciatic nerve. Spine (Phila Pa 1976). 2005;30(9):1008-13.

71. Van Boxem K, Huntoon M, Van Zundert J, Patijn J, van Kleef M, Joosten EA. Pulsed radiofrequency: a review of the basic science as applied to the pathophysiology of radicular pain: a call for clinical translation. Reg Anesth Pain Med. 2014;39(2):149-59.

72. Huang RY, Liao CC, Tsai SY, Yen CT, Lin CW, Chen TC, et al. Rapid and Delayed Effects of Pulsed Radiofrequency on Neuropathic Pain: Electrophysiological, Molecular, and Behavioral Evidence Supporting LongTerm Depression. Pain Physician. 2017;20(2):E269-E83.

73. Koopmans GC, Deumens R, Honig WM, Hamers FP, Steinbusch HW, Joosten EA. The assessment of locomotor function in spinal cord injured rats: the importance of objective analysis of coordination. J Neurotrauma. 2005;22(2):214-25.

74. Hamers FP, Lankhorst AJ, van Laar TJ, Veldhuis WB, Gispen WH. Automated quantitative gait analysis during overground locomotion in the rat: its application to spinal cord contusion and transection injuries. J Neurotrauma. 2001;18(2):187-201.

75. Hamers FP, Koopmans GC, Joosten EA. CatWalk-assisted gait analysis in the assessment of spinal cord injury. J Neurotrauma. 2006;23(3-4):537-48.

76. Koopmans GC, Brans M, Gomez-Pinilla F, Duis S, Gispen WH, Torres-Aleman I, et al. Circulating insulinlike growth factor I and functional recovery from spinal cord injury under enriched housing conditions. Eur $J$ Neurosci. 2006;23(4):1035-46.

77. Vrinten DH, Hamers FF. 'CatWalk' automated quantitative gait analysis as a novel method to assess mechanical allodynia in the rat; a comparison with von Frey testing. Pain. 2003;102(1-2):203-9.

78. Gabriel AF, Marcus MA, Walenkamp GH, Joosten EA. The CatWalk method: assessment of mechanical allodynia in experimental chronic pain. Behav Brain Res. 2009;198(2):477-80.

79. Neumann M, Wang Y, Kim S, Hong SM, Jeng L, Bilgen M, et al. Assessing gait impairment following experimental traumatic brain injury in mice. J Neurosci Methods. 2009;176(1):34-44.

80. Vlamings R, Visser-Vandewalle V, Koopmans G, Joosten EA, Kozan R, Kaplan S, et al. High frequency stimulation of the subthalamic nucleus improves speed of locomotion but impairs forelimb movement in Parkinsonian rats. Neuroscience. 2007;148(3):815-23.

81. Truin $M$, van Kleef $M$, Verboeket $Y$, Deumens $R$, Honig W, Joosten EA. The effect of Spinal Cord Stimulation in mice with chronic neuropathic pain after partial ligation of the sciatic nerve. Pain. 2009;145(3):312-8. 


\section{Appendix}

\section{Valorization}


Chronic neuropathic pain affects approximately $8 \%$ of the total adult human population and comes with significant burden for both the patient and healthcare system [1]. Patients often experience reduced functionality, which, in many cases results in them being uncapable of working. Chronic neuropathic pain is typically characterized by a low healthrelated quality of life; compared to other major diseases such as cancer, type-2 diabetes, and cardiovascular diseases, the health-related quality of life of patients with chronic neuropathic pain is significantly lower [2]. Because of the high prevalence of chronic neuropathic pain and the low health-related quality of life patients suffer from, chronic neuropathic pain forms a serious burden on both the healthcare system as well as the economy.

The effectiveness of pharmacological treatment of chronic neuropathic pain is often very limited, with less than $50 \%$ of patients responding to therapy. Furthermore, pharmacological interventions are often accompanied by unacceptable side effects such as development of tolerance and addiction [3]. It is therefore that further optimization of chronic neuropathic pain treatment is needed and it is in this context that the present thesis focusses on the role and mechanisms of action of interventional neuromodulatory approaches.

Interventional neuromodulation techniques such as spinal cord stimulation (SCS), dorsal root ganglion stimulation (DRGS) and pulsed radiofrequency (PRF) often provide a suitable therapy for refractory neuropathic pain patients [4-16]. Moreover, the side effects associated with these types of interventional treatments are typically less severe than those associated with many pharmacological treatment options [3]. However, despite the many advantages, these neuromodulation techniques do not come without limitations. A substantial group of patients treated with either SCS, DRGS or PRF still do not respond to treatment or experience a lack of treatment success over time. In this light it is of utmost importance to understand why, when and how chronic neuropathic pain patients do not respond to these treatments. A mechanism-based approach which allows understanding of the mechanisms of action of these neuromodulatory treatments might result in improved efficacy of these therapies. 
SCS with conventional settings (Con-SCS) has been successfully used to treat chronic neuropathic pain for over 50 years. Over the last decade, several new stimulation waveforms and new stimulation targets (such as DRGS) have been introduced to the field of SCS in order to further improve stimulation efficacy, while simultaneously eliminating some of the limitations with Con-SCS. One of the most prominent examples of innovation within the parameter space of SCS is the paresthesia-free Burst paradigm [17]. While indeed evidence suggests that Burst-SCS can yield superior pain relief compared to Con-SCS [17-19], especially related to the cognitive-affective dimensions of the pain experience, Burst-SCS still lacks the anatomic specificity (ability to stimulate difficult-to-reach areas such as the extremities and the groin) and stability (stable paresthesia intensity regardless of body position) of DRGS Based on this knowledge, summarized in Chapter 2, we set out to combine for the first time the advantage of a new stimulation paradigm with a new location of SCS in an animal model of painful diabetic peripheral neuropathy (PDPN) in Chapter 3: burst stimulation of the dorsal root ganglion. From these experiments we concluded that while the maximum amount of pain relief was comparable between Con-DRGS and Burst-DRGS, Burst-DRGS, but not ConDRGS, showed a delayed wash-out effect, which might have serious implications for optimal stimulation delivery of Burst-DRGS as well as battery life of the IPG in clinical practice. In line with this, preclinical observations have been extended to the clinic, where further clinical studies have been performed on the efficacy of Burst "microdosing", a paradigm that relies on the introduction of stimulation-off phases inbetween stimulation-on phases. From this it was concluded that Burst-SCS microdosing is as effective as standard Burst-SCS, while having significantly lower battery consumption (and thus fewer battery replacements for the pain patient) [20]. Whether or not such a microdosing approach can also be successfully utilized for Burst-DRGS remains to be studied. Interestingly, with the publication of our first preclinical study on Burst-DRGS in PDPN, one clinical study has been published utilizing BurstDRGS in neuropathic pain patients [21]. The authors found that at the end of the 18 months treatment period, $78 \%$ of patients preferred the Burst-DRGS protocol over the Con-DRGS protocol. Burst-DRGS is currently being assessed formally, however, in a RCT that is under way (ClinicalTrials.gov identifier: NCT03318250). 
Previous studies have indicated that Burst-SCS can be further optimized by adjusting relevant stimulation parameters, such as amplitude, in order to modulate the amount of charge delivered to the nervous system [22]. In Chapter 4, we titrated the optimal stimulation amplitude for Burst-DRGS to approximately $52 \%$ of the motor threshold (MT), and found a nonlinear relation between Burst-DRGS amplitude and pain behavior outcome. Combined with the results of Chapter 3, these results allowed us to further optimize Burst-DRGS to give a form of stimulation that delivers maximum pain relief, while at the same time minimizing battery consumption. As the chronic pain patient is likely to benefit from such an optimized treatment, future clinical studies should aim to verify these findings.

Besides the titration of optimal stimulation parameters, another important aspect for optimizing DRGS therapy is understanding its underlying mechanism of action. From the field of SCS we know that the presence of the neurotransmitter GABA in the dorsal horn of the spinal cord plays an important role in its mode of action [23-27]. This even led to translation to the clinic, where baclofen, а GABAв receptor agonist was used to rescue initial nonresponders to SCS, and turn them into responders [28, 29]. Similarly, gaining more insight into the mechanisms of action underlying DRGS might lead to improved treatment of the chronic neuropathic pain patient. While it was recently shown that DRGS is not likely to rely on the release of GABA in the dorsal horn of the spinal cord [30], it is possible that local, GABAergic signaling in the DRG is involved [31]. In Chapter 5, we therefore tested the hypothesis that a second GABAergic gate is responsible for the pain relieving effect of DRGS. Based on our immunohistochemical findings, we found no evidence for such a GABAergic gate located in the DRG. Still, we consider the presentation of these data to be of great importance, as publishing bias (skewed towards positive findings) is an important problem that the academic community has to deal with. Based on this work, we can now look for alternative modes of action of DRGS to further improve DRGS therapy for the pain patient.

In the last part of the thesis (Chapter 6) the efficacy of a PRF treatment adjacent to the DRG on experimental chronic neuropathic pain is investigated and described. Fundamentally, PRF greatly differs from DRGS in the way it is delivered (once vs continuous 
delivery of current), something that is also expressed in the differences between the duration and amount of pain relief obtained with these two therapies. We showed that treatment with PRF to the DRG significantly attenuated mechanical and thermal hypersensitivity, albeit to lesser extent than DRGS (Chapter 3 and 4), whereas no effect was observed on painrelated gait using the CatWalk system. With our study, previous findings [32] related to the pain relieving effect of PRF were confirmed, something which is important when establishing a reproducible and valid animal model for studying the effects end mechanisms of action of a given treatment. At the same time, our, and previous [33] data strongly suggests that CatWalk gait analysis does not allow to detect or analyze behavioral effects of interventional treatment approaches such as PRF and SCS in the chronic experimental PSNL model. Nevertheless, studying behavioral effects (short or long-lasting) is still an issue in the experimental pain field and may make the impact of pain relieving effects and the interpretation and translation of findings to the clinic very difficult. To date, reflex-based outcome measures such as mechanical hypersensitivity (e.g. Von Frey test) and thermal hypersensitivity (e.g. Hargreaves test) are considered to be the golden standard in experimental pain research. However, as these tests are rather subjective and rely on evoked nociception instead of spontaneous pain, they are not optimal. Future studies should therefore aim to include more operant-like tests that also take into account the affectiveemotional and cognitive aspects of pain in order to improve the translational significance of preclinical observations.

From a practical point of view, the results presented in this academic thesis provide strong arguments for continuing experimental research on the efficacy and mechanisms of action of neuromodulation of the DRG in experimental chronic neuropathic pain. This should then be based on an orchestrated interplay between reproducible experimental animal studies and well-designed large, (preferably) non-industry initiated clinical trials. Given the fundamental differences in terms of efficacy and duration of pain relief between DRGS and PRF, they are very likely to depend on different mechanisms of action. By gaining more insight into these mechanisms of action, the efficacy of interventional pain treatments for the 
chronic neuropathic pain patient might significantly increase, thereby reducing the societal and economic burden of chronic neuropathic pain as a disease. 


\section{References}

1. International Association for the Study of Pain (Retrieved 9 June 2016).

2. Van Boxem K, Huntoon M, Van Zundert J, Patijn J, van Kleef M, Joosten EA. Pulsed radiofrequency: a review of the basic science as applied to the pathophysiology of radicular pain: a call for clinical translation. Reg Anesth Pain Med. 2014;39(2):149-59.

3. Finnerup NB, Otto $M$, Jensen TS, Sindrup SH. An evidence-based algorithm for the treatment of neuropathic pain. MedGenMed. 2007;9(2):36.

4. de Vos CC, Meier K, Zaalberg PB, Nijhuis HJ, Duyvendak W, Vesper J, et al. Spinal cord stimulation in patients with painful diabetic neuropathy: a multicentre randomized clinical trial. Pain. 2014;155(11):2426-31.

5. Deer TR, Levy RM, Kramer J, Poree L, Amirdelfan K, Grigsby E, et al. Dorsal root ganglion stimulation yielded higher treatment success rate for complex regional pain syndrome and causalgia at 3 and 12 months: a randomized comparative trial. Pain. 2017;158(4):669-81.

6. Eldabe S, Burger K, Moser H, Klase D, Schu S, Wahlstedt A, et al. Dorsal Root Ganglion (DRG) Stimulation in the Treatment of Phantom Limb Pain (PLP). Neuromodulation. 2015;18(7):610-6; discussion 6-7.

7. Eldabe S, Espinet A, Wahlstedt A, Kang P, Liem L, Patel NK, et al. Retrospective Case Series on the Treatment of Painful Diabetic Peripheral Neuropathy With Dorsal Root Ganglion Stimulation. Neuromodulation. 2018;21(8):787-92.

8. Huygen F, Liem L, Cusack W, Kramer J. Stimulation of the L2-L3 Dorsal Root Ganglia Induces Effective Pain Relief in the Low Back. Pain Pract. 2018;18(2):205-13.

9. Kumar K, Taylor RS, Jacques L, Eldabe S, Meglio M, Molet J, et al. The effects of spinal cord stimulation in neuropathic pain are sustained: a 24-month follow-up of the prospective randomized controlled multicenter trial of the effectiveness of spinal cord stimulation. Neurosurgery. 2008;63(4):762-70; discussion 70.

10. Liem L, Russo M, Huygen FJ, Van Buyten JP, Smet I, Verrills P, et al. A multicenter, prospective trial to assess the safety and performance of the spinal modulation dorsal root ganglion neurostimulator system in the treatment of chronic pain. Neuromodulation. 2013;16(5):471-82; discussion 82.

11. North RB, Kumar K, Wallace MS, Henderson JM, Shipley J, Hernandez J, et al. Spinal cord stimulation versus re-operation in patients with failed back surgery syndrome: an international multicenter randomized controlled trial (EVIDENCE study). Neuromodulation. 2011;14(4):330-5; discussion 5-6.

12. Slangen R, Schaper NC, Faber CG, Joosten EA, Dirksen CD, van Dongen RT, et al. Spinal cord stimulation and pain relief in painful diabetic peripheral neuropathy: a prospective two-center randomized controlled trial. Diabetes Care. 2014;37(11):3016-24.

13. Teixeira A, Grandinson M, Sluijter ME. Pulsed radiofrequency for radicular pain due to a herniated intervertebral disc--an initial report. Pain Pract. 2005;5(2):111-5.

14. Van Boxem K, de Meij N, Kessels A, Van Kleef M, Van Zundert J. Pulsed radiofrequency for chronic intractable lumbosacral radicular pain: a six-month cohort study. Pain Med. 2015;16(6):1155-62.

15. Van Boxem K, van Bilsen J, de Meij N, Herrler A, Kessels F, Van Zundert J, et al. Pulsed radiofrequency treatment adjacent to the lumbar dorsal root ganglion for the management of lumbosacral radicular syndrome: a clinical audit. Pain Med. 2011;12(9):1322-30.

16. Van Zundert J, Patijn J, Kessels A, Lame I, van Suijlekom H, van Kleef M. Pulsed radiofrequency adjacent to the cervical dorsal root ganglion in chronic cervical radicular pain: a double blind sham controlled randomized clinical trial. Pain. 2007;127(1-2):173-82.

17. De Ridder D, Vanneste S, Plazier M, van der Loo E, Menovsky T. Burst spinal cord stimulation: toward paresthesia-free pain suppression. Neurosurgery. 2010;66(5):986-90.

18. De Ridder D, Lenders MW, De Vos CC, Dijkstra-Scholten C, Wolters R, Vancamp T, et al. A 2-center comparative study on tonic versus burst spinal cord stimulation: amount of responders and amount of pain suppression. Clin J Pain. 2015;31(5):433-7.

19. De Ridder D, Plazier M, Kamerling N, Menovsky T, Vanneste S. Burst spinal cord stimulation for limb and back pain. World Neurosurg. 2013;80(5):642-9 e1.

20. Vesper J, Slotty P, Schu S, Poeggel-Kraemer K, Littges H, Van Looy P, et al. Burst SCS Microdosing Is as Efficacious as Standard Burst SCS in Treating Chronic Back and Leg Pain: Results From a Randomized Controlled Trial. Neuromodulation. 2019;22(2):190-3.

21. Al-Kaisy A, Royds J, Costanzi M, Racz G, Wesley S, Palmisani S, et al. Effectiveness of "Transgrade" Epidura Technique for Dorsal Root Ganglion Stimulation. A Retrospective, Single-Center, Case Series for Chronic Foca Neuropathic Pain. Pain Physician. 2019;22(6):601-11. 
22. Crosby ND, Goodman Keiser MD, Smith JR, Zeeman ME, Winkelstein BA. Stimulation parameters define the effectiveness of burst spinal cord stimulation in a rat model of neuropathic pain. Neuromodulation. 2015;18(1):1-8; discussion

23. Cui JG, Meyerson BA, Sollevi A, Linderoth B. Effect of spinal cord stimulation on tactile hypersensitivity in mononeuropathic rats is potentiated by simultaneous GABA(B) and adenosine receptor activation. Neurosci Lett. 1998;247(2-3):183-6.

24. Cui JG, O'Connor WT, Ungerstedt U, Linderoth B, Meyerson BA. Spinal cord stimulation attenuates augmented dorsal horn release of excitatory amino acids in mononeuropathy via a GABAergic mechanism. Pain. 1997;73(1):87-95.

25. Janssen SP, Gerard S, Raijmakers ME, Truin M, Van Kleef M, Joosten EA. Decreased intracellular GABA levels contribute to spinal cord stimulation-induced analgesia in rats suffering from painful peripheral neuropathy: the role of KCC2 and GABA(A) receptor-mediated inhibition. Neurochem Int. 2012;60(1):21-30.

26. Linderoth B, Stiller CO, Gunasekera L, O'Connor WT, Ungerstedt U, Brodin E. Gamma-aminobutyric acid is released in the dorsal horn by electrical spinal cord stimulation: an in vivo microdialysis study in the rat. Neurosurgery. 1994;34(3):484-8; discussion 8-9.

27. Stiller CO, Cui JG, O'Connor WT, Brodin E, Meyerson BA, Linderoth B. Release of gamma-aminobutyric acid in the dorsal horn and suppression of tactile allodynia by spinal cord stimulation in mononeuropathic rats. Neurosurgery. 1996;39(2):367-74; discussion 74-5.

28. Lind G, Schechtmann G, Winter J, Meyerson BA, Linderoth B. Baclofen-enhanced spinal cord stimulation and intrathecal baclofen alone for neuropathic pain: Long-term outcome of a pilot study. Eur J Pain. 2008;12(1):1326.

29. Schechtmann G, Lind G, Winter J, Meyerson BA, Linderoth B. Intrathecal clonidine and baclofen enhance the pain-relieving effect of spinal cord stimulation: a comparative placebo-controlled, randomized trial. Neurosurgery. 2010;67(1):173-81.

30. Koetsier E, Franken G, Debets J, Heijmans L, van Kuijk SMJ, Linderoth B, et al. Mechanism of dorsal root ganglion stimulation for pain relief in painful diabetic polyneuropathy is not dependent on GABA release in the dorsal horn of the spinal cord. CNS Neurosci Ther. 2020;26(1):136-43.

31. Du X, Hao H, Yang Y, Huang S, Wang C, Gigout S, et al. Local GABAergic signaling within sensory ganglia controls peripheral nociceptive transmission. J Clin Invest. 2017;127(5):1741-56.

32. Vuka I, Vucic K, Repic T, Ferhatovic Hamzic L, Sapunar D, Puljak L. Electrical Stimulation of Dorsal Root Ganglion in the Context of Pain: A Systematic Review of In Vitro and In Vivo Animal Model Studies. Neuromodulation. 2018;21(3):213-24.

33. Truin $M$, van Kleef $M$, Verboeket $Y$, Deumens $R$, Honig W, Joosten EA. The effect of Spinal Cord Stimulation in mice with chronic neuropathic pain after partial ligation of the sciatic nerve. Pain. 2009;145(3):312-8. 


\section{Appendix}

Curriculum Vitae 
Glenn Franken was born in Brunssum, The Netherlands, on March 26th, 1992. He attended secondary school at Rombouts College in Brunssum, where he obtained his Atheneum degree in 2010. That same year, he started his Bachelor in Biomedical Sciences, with a specialization in Molecular Life Sciences (Minors: Neurosciences and Toxicology, Diseased Cells), at Maastricht University. During his Bachelor internship, Glenn studied the effect of exercise training on intrahepatic lipid content in type 2 diabetic men by means of MRI under supervision of Dr. Bram Brouwers and Prof. Dr. Patrick Schrauwen at the Department of Human Biology. He obtained his Bachelor degree in 2013, after which he started his Master in Biomedical Sciences. During his Junior Master Internship, he joined the research team 'Neuroinflammation and Autoimmunity' supervised by Prof. Dr. Pilar Martinez-Martinez at Maastricht University. Here, Glenn studied the presence of autoantibodies against the $\mathrm{N}$-methyl-D-aspartate (NMDA) receptor in schizophrenic patients. For his Senior Master Internship, Glenn joined the Department of Anesthesiology and Pain Management, supervised by Prof. Dr. Bert Joosten, to study the effect of Pulsed Radiofrequency (PRF) treatment on experimental chronic neuropathic pain. After successfully completing his Master studies in 2015, Glenn started his PhD project at this same department under supervision of Prof. Dr. Bert Joosten, Prof. Dr. Jan van Zundert, and Prof. Dr. Liong Liem. During his PhD, Glenn studied the efficacy and mechanisms of action of neuromodulation of the dorsal root ganglion (DRG), in particular PRF and dorsal root ganglion stimulation (DRGS), for the treatment of experimental chronic neuropathic pain. He collaborated with many experts in the field of neuromodulation and pain, including Prof. Dr. Bengt Linderoth (Karolinska Institutet, Stockholm, Sweden), Prof. Dr. Quinn Hogan (University of Wisconsin, USA), Prof. Dr. Roberto Perez (VUMC, Amsterdam, Netherlands), as well as world leading industry partners in the neuromodulation field (Abbott Laboratories inc.). The results of his studies are presented in this academic thesis. Following his PhD defense, Glenn will continue his scientific career as a postdoctoral researcher at the Department of Anesthesiology and Pain Management at Maastricht University. 


\section{Appendix}

List of Publications 
2020 Joosten EA, Franken G. Spinal Cord Stimulation in Chronic Neuropathic Pain: Mechanisms of Action, New locations, New Paradigms. Pain. 2020;161 Suppl 1:S104-S13. Published.

2019 Franken G, Debets J, Joosten EAJ. Dorsal Root Ganglion Stimulation in Experimental Painful Diabetic Peripheral Neuropathy: Burst vs. Conventional Stimulation Paradigm. Neuromodulation. 2019;22(8):943-50. Published.

2020 Franken G, Debets J, Joosten EAJ. Nonlinear Relation Between Burst Dorsal Root Ganglion Stimulation Amplitude and Behavioral Outcome in an Experimental Model of Painful Diabetic Peripheral Neuropathy. Neuromodulation. 2020;23(2):158-66. Published.

2020 Franken G, Douven P, Debets J, Steinbusch He, Joosten EAJ. A Possible Mechanism of Conventional Dorsal Root Ganglion Stimulation in Pain Relief: Quantitative Immunocytochemical Analysis of Intracellular GABA in DRG Neurons. Submitted.

2020 Franken G, Joosten EA. The Use of CatWalk Gait Analysis for Assessing Chronic Neuropathic Pain in Rats Treated with Pulsed Radiofrequency. Submitted.

2019 Koetsier E, Franken G, Debets J, van Kuijk SMJ, Perez R, Linderoth B, et al. Effectiveness of Dorsal Root Ganglion Stimulation and Dorsal Column Spinal Cord Stimulation in a Model of Experimental Painful Diabetic Polyneuropathy. CNS Neurosci Ther. 2019;25(3):367-74. Published.

2020 Koetsier E, Franken G, Debets J, Heijmans L, van Kuijk SMJ, Linderoth B, et al. Mechanism of Dorsal Root Ganglion Stimulation for Pain Relief in Painful Diabetic Polyneuropathy is Not Dependent on GABA Release in the Dorsal Horn of the Spinal Cord. CNS Neurosci Ther. 2020;26(1):136-43. Published.

2020 Koetsier E, Franken G, Debets J, van Kuijk SMJ, Linderoth B, Joosten EA, et al. Dorsal Root Ganglion Stimulation in Experimental Painful Diabetic Polyneuropathy: Delayed Wash-Out of Pain Relief After Low-Frequency $(1 \mathrm{~Hz})$ Stimulation. Neuromodulation. 2020;23(2):177-84. Published. 


\section{Appendix}

Dankwoord 
Allereerst mijn promotor en dagelijkse supervisor Prof. Dr. Bert Joosten. Bert, in 2014 begon ik aan mijn stage periode binnen de befaamde "pijn groep". Na een kleine miscommunicatie (ik was er van overtuigd dat de stage 1 november zou beginnen, maar dit bleek toch al een week eerder te zijn) klikte het tussen ons toch al vrij snel, en het duurde dan ook niet lang voordat de gesprekken begonnen over een mogelijk aansluitend promotie traject welke in 2016 van start is gegaan. Vier jaar later is het dan zo ver, en ik kan zeggen dat ik geen moment spijt heb gehad van deze keuze. Jouw natuurlijke talent om een groep van gezellige Limburgers en Brabanders bij elkaar te brengen, aangevuld met een sprankje jong, exotisch talent uit de noordelijkere regionen, heeft een groep bij elkaar gebracht waar we denk ik trots op mogen zijn. Vooral het aantal contacturen met je PhD studenten die je, ondanks je drukke agenda, eruit weet te persen verdienen credits. Gedurende mijn PhD ben ik gegroeid zowel als onderzoeker en als mens, waarvoor dank!

Mijn tweede promotor, Prof. Dr. Jan van Zundert; Beste Jan, hoewel onze échte samenwerking pas vrij recentelijk is gestart sinds jouw benoeming als hoofd van de pijn afdeling, ben ik in mei 2015 als "broekie" al eens langs geweest in Lanaken om een aantal PRF ingrepen bij te wonen. Dat je mijn bezoek destijds, later verwarde met Koen (Meuwissen) vergeef ik je bij deze. Jouw openheid en transparantie waardeer ik zeer, evenals de translationele aanpak van jouw leidinggeven. Het is ook niet voor niets dat we vanaf 1 september gezamenlijk een mooi postdoc project zijn gestart! Ik kijk uit naar onze verdere samenwerking.

Mijn co-promotor, Prof. Dr. Liong Liem. Beste Liong, hoewel onze agenda's niet altijd matchten (Liong is een echte wereldbewoner; je weet nooit waar hij deze keer weer uithangt), was jij altijd bereikbaar en in voor een belletje of "appje". Ik heb veel geleerd van jouw klinische ervaring en kritische houding wat betreft DRG stimulatie. Daarnaast is een meeting met jou ook nooit saai; ik vergeet nooit meer dat tijdens het maken van een presentatie plotseling een kerkklok afging op jouw telefoon omdat dit je aan thuis liet denken. 
Daarnaast wil ik ook Prof. Dr. Maarten van Kleef bedanken. Beste Maarten, gedurende jouw periode als hoofd van de pijnafdeling heb ik jouw ongezouten, Rotterdamse mening en objectiviteit altijd zeer gewaardeerd. Het is me dan ook een eer dat jij deel zal nemen in mijn Corona. Geniet van je welverdiende pensioen, al heb ik het gevoel dat wij met enige regelmaat nog wat van jou zullen vernemen. Een arts en onderzoeker in hart en nieren.

My paranymfs, Margot and Chris. Margot, allereerst wil ik je een compliment geven over hoe jij je als erkende thee connaisseur staande weet te houden binnen een divisie die zo koffie gedreven is. Na een kleine acclimatisatie periode (lees: Van der Valk) denk ik dat we toch kunnen stellen dat het meer dan goed gekomen is tussen ons, zowel op de werkvloer als privé. Ook al vinden onze wekelijkse thee meetings slechts zeer sporadisch plaats, wordt dit meer dan voldoende gecompenseerd door de vaak diepe (en raadgevende) gesprekken. Kwaliteit boven kwantiteit zullen we maar zeggen! Ik waardeer jouw down-to-earth, maar positieve houding enorm en weet zeker dat wij ook buiten het werk om contact zullen houden. Je weet nu immers dat ik toch wel (een beetje) kan koken;). Chris, you are without a doubt one of the most social persons that I know. The positivity you bring to the work floor is inspiring. You are always concerned with other people, and sometimes appear to be a walking encyclopedia; your natural ability to remember things (be it birthdays, holidays, or where I was 2 years ago on a random Thursday) is remarkable. I am sure we will have this shisha in the near future; but, like I said before, planning is important. P.S. If you are searching for Chris, your best chance is to look for him in the corridor of our division, as there is a high likelihood that you will find him roaming around searching for somebody to drink coffee (or have "lunch") with;).

Onderzoek is een team effort, en daarom wil ik zeker ook de leden van de pijngroep bedanken. Roel, ondanks dat we af en toe anders over zaken denken (en nee ik doel niet op flat earth), ben ik ervan overtuigd dat wij in de loop der jaren een zeer goede band hebben opgebouwd. Ik wens je alvast heel veel succes bij je nieuwe baan. Voordeel voor mij is dat nu in ieder geval niet mijn kantoor plek aan het raam wordt ingepikt;). Rose, "ex-roomie", ik was er misschien niet vaak, maar ik heb daarom niet minder genoten van alle etentjes samen 
met jou, Perla en Toon. Mocht je Perla (of mij) missen, jij blijft altijd welkom! Lonne, je was mijn eerste student, dus wat dat betreft bevond ik me destijds ook in een pilot fase. Ik ben in ieder geval trots dat jij later ons team bent komen versterken! Je hebt een goede, hardwerkende instelling die ik enorm waardeer. Martijn, ik kan nu bepaalde anekdotes erin gaan gooien, maar die laat ik even voor wat het is (op request wil ik deze echter zeker vrijgeven). Jouw open houding en behulpzaamheid zie je niet vaak, en ik kijk uit naar onze verdere samenwerking wat betreft DRGS onderzoek. Thomas, jij bent altijd in voor een goede discussie. Ik hoop dat je mijn "geplaag" altijd in de juiste context hebt kunnen zien (ik denk het wel). Rotterdammers hebben soms gewoon wat Limburgs tegengas nodig! Jacques, Wiel heeft in jou een uitstekende "opvolger" weten te regelen. Ik weet zeker dat de pijngroep (en inmiddels de hele neuromodulatie expert group) bij jouw kunde en expertise gebaat is. Al vind ik om half 8 beginnen met implantaties nog steeds erg vroeg;).

Daarnaast wil ook een aantal oud collega's van het pijnteam bedanken, te beginnen met Koen. Koen, thank you for all your kindness and support, moreover..... Oké even opnieuw: Koen, ik doe jou eigenlijk tekort met enkel een beschrijving als "oud-collega". Jij hebt, naast je vaardigheden als wetenschapper, op meerdere vlakken een positieve invloed op me gehad: je introduceerde me tot boksen (maar dan enkel kijken, want daadwerkelijk tegen Koen boksen wil je niet... ik spreek uit ervaring), en mijn deur zal zeker voor je open staan voor Fury-Wilder 3. Mits jij en Marina zelf pizza en drank meenemen. Daarnaast kregen jij en Roy me zowaar aan t sporten, waarvoor dank! En we blijven het gewoon stug volhouden; Dublin is géén mooie stad. Al zijn er altijd manieren om het een stuk aangenamer te maken;). Nynke, dankzij jou weet ik nu hoe een promotie achter de schermen er aan toe gaat! Ik hoop dat je het naar je zin hebt in Canada, en we drinken er snel nog eens eentje wanneer je terug in Maastricht bent. Maarten, met jou was het nooit saai op kantoor, al had ik soms lichte angst om je aan te spreken wanneer je weer eens "zo'n" bui had;) Ik hoop dat je je draai gevonden hebt zowel op werkvlak als privé. P.S. Vergeet niet dat ik je van een elektrocutie gered heb!: you owe me one! Wiel, jij was er vanaf het begin van mijn PhD bij, en was er altijd om onze groep uit de brand te helpen. Geniet van je welverdiende pensioen, hopelijk kun je na al deze ellende weer snel met de camper naar Marokko! 
Daarnaast zijn er nog een aantal collega's die ik graag wil bedanken. Sylvana, jij bent de definitie van een power woman; nuchter en principieel. Ik heb je altijd zeer gewaardeerd zowel als persoon en als wetenschapper. Ik vind het dan ook erg jammer (maar begrijpelijk) dat je Maastricht hebt verlaten. Ik hoop dat jij en Wesley snel een nieuw plekje vinden, en ik wens je heel veel succes bij je nieuwe baan! Ik verwacht binnen een niet al te lange tijd dan ook zeker een uitnodiging te ontvangen voor een housewarming;). Fred, bedankt voor jouw altijd kritische inzichten wat betreft horloges. Ik weet zeker dat ik me vast nog een keer laat verleiden tot een (te dure) aankoop wanneer je terug bent (misschien een rattrapante?). $42 \mathrm{~mm}$ is té groot. Veel succes in the USA! Marina, small girl, great personality! I wish you all the best finishing up your own thesis. I will make sure to have the Disaronno ready afterwards! I am sure that Koen and I will gently force you and Perla to watch another boxing match in the near future. Wouter, van alle mensen op de afdeling ken ik jou het langst. Je blijft altijd dicht bij jezelf staan, wat ik waardeer. De enige keer die ik me kan herinneren dat wij onenigheid hebben gehad was de keer dat je ons complete verslag (per ongeluk) besloot te verwijderen. Het is uiteindelijk toch allemaal goed gekomen;). Heel veel succes met het afronden van je eigen onderzoek.

Daarnaast dank aan ons technician team: Hellen, Denise, Wouter, Sandra, Barbie, maar ook oud collega's Marjan, Rachelle, Marcella en Anne; bedankt voor jullie harde inzet en ondersteuning! Ook dank aan het hele CPV team: Richard, Saskia, Clarice, Rik, Paul, Harry, Mandy en alle andere medewerkers voor hun support.

Tot slot wil ik ook alle overige collega's van het lab bedanken: Maite, Clara, Katherine, Alix, Ghazi, Faisal, Faris, Mohammed, Roman, Jeroen, Ralph, Christian, Renzo, Janou, Philippos, Dean, Ellis, Manon, Gusta, Jana, Aimee, Shengua, Carolin, Nikita, Tanya, Caterina, Daan, Jackson, Thijs, Nasim, Christian, Sarah, Michiel, Jos, David, Bart, Pilar, Daniel, Ali, Govert, Mario, Eshan, Gunter, Lars, Laurence, Tim, Rachelle, Marie-Therese, Nicole, Ankie, Tom, alsmede alle collega's uit de kliniek: Nelleke, Mischa, Wolfgang, Xander, Sander, Dianne, Maurice, Anne, Brigitte, Andrea, Philma, Carine, Veerle, José, Daisy, Dion, Thibaut, en iedereen die ik onverhoopt vergeten te noemen ben. 
I also would like to thank our colleagues abroad. Erika Ross, David Zhang, Lalit Venkatesan, Alexander Kent, Bill Cusack, Jeffery Kramer at Abbott Laboratories; thank you very much for your continued support and collaboration! Also thanks to Quinn Hogan and Bin Pan for hosting Wiel and myself back in 2016 and training us in the DRGS implantation. I could not have finished this research were it not for you. Also thank you to Eva Koetsier, Paolo Maino, Roberto Perez, and Bengt Linderoth for all the fruitful collaborations.

Vanzelfsprekend wil ik graag ook de mensen buiten het werk bedanken die mij gedurende de afgelopen jaren hebben gesteund. Ilvy, buurvrouw, met jou kan ik altijd levelen wat betreft lekker eten: met name sushi, frieten en frikandellen. Maar voordat de lezer nu denkt "wat zijn deze mensen ongezond" wil ik bij deze toch ook onze (soms wat wisselvallige) mountainbike carrière benadrukken. Cheryl, uit het oog maar niet uit het hart. Er zijn weinig mensen die zo'n aanstekelijke lach als jij hebben. Ik kijk met veel plezier terug op alle treinritjes en beschamende confrontaties met de kiosk mevrouw. Ik hoop jou en Caspar snel weer te zien, want ik begin jullie gekibbel tijdens de spelavondjes inmiddels toch wel te missen;). Veel succes met het afronden van jullie eigen thesis, en ik hoop jullie snel terug te zien in Maastricht! Jory en Dylan, bedankt voor alle mooie momenten. Wij laten denk ik zien dat je elkaar niet wekelijks hoeft te zien om een goede band te onderhouden. Ik kijk nu al uit naar de volgende Star Wars film, zodat Dylan nog maar eens zijn geweldige Chewbacca imitatie kan laten zien;). Anniek, jij bent een van de liefste mensen die ik ken. Ik ben blij dat je inmiddels je eigen plekje hebt gevonden, en ik kijk uit naar alle toekomstige bbq's en feestmomentjes samen. Maar dan wel mét pina colada shirt;). Anke, soms spreken we elkaar maanden niet, soms elke dag. Desalniettemin vervelen wij ons nooit samen. Bedankt voor alle koffiemomentjes, al moeten we soms creatief zijn gedurende deze unieke omstandigheden;). Melissa, "Mel", jij bent een reddende engel op het moment dat Perla op vakantie is. Jouw gezelligheid wordt altijd zeer gewaardeerd, al is het maar om samen de persconferentie van Mark Rutte te kijken. Ook wil ik de "Masters of Science 2015" bedanken: Glenn R, Aaron, Bas \& Bas, Pauline, Rianne, Wouter. Het is altijd leuk om deze "oude gezichten" opnieuw te zien. Speciale dank aan Glenn R die er altijd keurig voor zorgt dat er toch zeker jaarlijks een meeting wordt gepland. Daarnaast dank aan Henk, Ria, Yanick en 
Lotte; Ik hoop dat er nog veel leuke weekendjes weg volgen. Al betwijfel ik of zo'n geslaagd uitje (met zoveel bier en wijn) nog wel te evenaren is.

Als laatste wil ik mijn gehele familie bedanken, en in het bijzonder de mensen die het dichtst bij me staan: Kimberly, "zusje", ik hoop dat je na mijn promotie toch iets meer van je broertje gaat aannemen. Al wordt wel eens gezegd dat succes ook naar je hoofd kan stijgen... Ik ben enorm trots op jou als zus! Tom, dé redder in nood (samen met Bram) op de momenten dat je je huis niet meer uit komt. Jij bent precies wat Kimberly nodig heeft, en een topper in hart en nieren. Ik zou nu ook graag de naam van mijn toekomstige neefje noemen, maar dit is "the best kept secret" binnen de families Offermans en Franken;). Hoe dan ook, ik kan niet wachten om mijn neefje, jullie kind, snel te verwelkomen!

Papa en mama, heb ik toch maar even mooi gedaan he? Ik heb jullie vaak horen zeggen "Glenn doet dat wel even". Maar het feit is dat ik deze Dr. titel nooit gehaald zou hebben zonder jullie onvoorwaardelijke steun; zonder jullie zou ik niet staan waar ik nu sta. Pap, jij herkent veel van jezelf in mij. Je hebt me geleerd om de ideale balans te vinden tussen hard werken en "chillen". Dat laatste uit zich in onze gezamenlijke passie voor films (en tegenwoordig zelfs financiën, wie had dat ooit gedacht??), en ik hoop dan ook dat we nog vele bioscoop avonden samen mogen doorbrengen! Ik hoop dat je samen met mama en ons enorm geniet van je pensioen. Je hebt het verdiend! Mam, het klinkt misschien cliché, maar ik ben ervan overtuigd dat wij door hetgeen wij samen hebben meegemaakt een unieke band hebben. Nog steeds ben jij er altijd voor me wanneer dat nodig is, zowel op de écht kritische momenten, als om vragen over de wasmachine te beantwoorden. Niets is voor jou te gek, en je staat binnen een paar minuten voor de deur als dat moet. Je hebt me geleerd om altijd mijn hart te volgen, of dat nu privé of op het werk is. Als jij maar half zo trots op mij bent als ik op jou, ben ik meer dan tevreden.

Lieve Perla, omdat je met enige regelmaat aangeeft dat je het dialect spreken zo erg mist heb ik mijn uiterste best gedaan om dit stukje in authentiek Ni-jwieërts dialect te schrijven. Wae zeen net gaon samewoeene, des toch unne hieele stap. Trök kieekendj deenk ich det de aafrondingsfase van mieen PhD dan aug un ideale proefperiode is gewaesj. Dich 
hes mich tieedes deze tieed ontzettendj geholpe met ut aafronde van mieen leste experimente en dit bukske, en dich hes de gave um rust te breenge in mieen (meistal zelf gecrejeerde) chaos. Op un paar stress momente nao deenk ich det we toch kinne stelle det wae met vlag met wimpel geslaagdj zeen;). Ich wil dich bedanke veur alles, en ich weit zeker det wae nog vul leuke jaore tegemoot gaon same in os 'nowwe' appartement. Al mot det hundje toch echt nog ff wachte! 
